Universidade de São Paulo

Instituto de Física

\title{
Estudo da coincidência elétron-jato em colisões próton-próton e próton-núcleo no experimento ALICE
}

\author{
Diógenes Domenicis Gimenez
}

Orientador: Prof. Dr. Marcelo Gameiro Munhoz

Tese submetida ao Instituto de Física da Universidade de São Paulo para a obtenção do título de Doutor em Ciências

Banca Examinadora:

Prof. Dr. Marcelo Gameiro Munhoz - Orientador (IFUSP)

Prof $^{a}$. Dra ${ }^{a}$. Frederique Grassi (IFUSP)

Prof. Dr. Marco Aurélio Lisboa Leite (IFUSP)

Prof ${ }^{\mathrm{a}}$. Dr ${ }^{\mathrm{a}}$. Sandra dos Santos Padula (IFT UNESP)

Prof ${ }^{\mathrm{a}}$. Dr ${ }^{\mathrm{a}}$. Maria Beatriz Leone Gay (UFRGS) 


\section{FICHA CATALOGRÁFICA}

Preparada pelo Serviço de Biblioteca e Informação do Instituto de Física da Universidade de São Paulo

Gimenez, Diógenes Domenicis

Estudo da coincidência elétron-jato em colisões prótron-próton e próton-núcleo no experimento ALICE. São Paulo, 2018.

Tese (Doutorado) - Universidade de São Paulo. Instituto de Física. Depto. de Física Nuclear

Orientador: Prof. Dr. Marcelo Gameiro Munhoz

Área de Concentração: Física de Alta Energia

Unitermos: 1. Quark; 2. Elétrons;3. Física nuclear. 
University of São Paulo

Physics Institute

\title{
Measurement of the electron-jet coincidence in proton-proton and proton-nucleus collisions with ALICE
}

\author{
Diógenes Domenicis Gimenez
}

Supervisor: Prof. Dr. Marcelo Gameiro Munhoz

Thesis submitted to the Physics Institute of the University of São Paulo in partial fulfillment of the requirements for the degree of Doctor of Science.

Examining Commitee:

Prof. Dr. Marcelo Gameiro Munhoz - Supervisor (IFUSP)

Prof. Dr. Frederique Grassi (IFUSP)

Prof. Dr. Marco Aurélio Lisboa Leite (IFUSP)

Prof. Dr. Sandra dos Santos Padula (IFT UNESP)

Prof. Dr. Maria Beatriz Leone Gay (UFRGS) 

"A new scientific truth does not triumph by convincing its opponents and making them see the light, but rather because its opponents eventually die, and a new generation grows up that is familiar with it."

- Max Planck 



\section{Acknowledgments}

I would like to thank all the people who worked with me, taught me, supported me and bore me during this last five years. In special, I would like to thank my supervisor Marcelo Gameiro Munhoz, who guided me through the complex paths and motivated me when it was needed.

I would like also to thank my father Marco Antonio Julca Gimenez and my mother Ana Maria Domenicis Gimenez, who have always supported me emotionally and financially, and have always believed in my choices. They are also responsible for all my achievements. I cannot forget my sister, Giovana Domenicis Gimenez, who shared with me all the good and bad moments, and helped me in finding solutions for many problems, related or not to the Ph.D.

I would like to acknowledge the great supporting and teaching provided by my friends in the Physics Institute, since undergraduation: Everton Arrighi, Jessica Santiago Silva, Matheus Lopes Rodrigues, Lucas Medeiros Cornetta, Pedro Henrique Ormonde Silva, Xinxin Zhang. And also the friends I made in the graduation: Antonio Carlos Oliveira da Silva, Arthur Lopes, Caio Prado, Camila Conti, Cristiane Jahnke, Henrique José Correia Zanoli, Hermann Degenhardt, Marcel Figueiredo and Pedro Hugo Natal da Luz.

I would like to thank the professors in HEPIC group Alexandre Alarcon do Passo Suaide, Marco Bregant and Mauro Cosentino, whose suggestions and critics are part of this analysis.

I would like to thank Antonio for his help guiding me in Switzerland and teaching me virtualy in every step of the analysis. His comments and lessons were imperative.

I would like to thank Henrique, my friend since the undergraduation, for his advices 
and for finding practical solutions for my problems.

I would like to thank Luca Menichetti, Alessandra Ditta and Camila Sampaio Machado, who helped me in keeping my sanity during the time at CERN.

I would like to thank Diógenes Novais da Cruz for existing and being my alter ego. His presence made life easier and worth living.

I would like to thank FAPESP (grant \#2013/05150-0, São Paulo Research Foundation - FAPESP) for its financial support to this work in Brazil and CNPq for the financial support at CERN. 


\section{Resumo}

Esta tese apresenta um estudo inédito da distribuição de jatos correlacionados com elétrons provenientes de decaimentos de quarks pesados (HFe), reconstruídos e selecionados com o ALICE (A Large Ion Collider Experiment, em inglês). Os observáveis foram medidos em colisões pp com energia do centro de massa $\sqrt{s}=8 \mathrm{TeV}$ e em colisões p-Pb a $\sqrt{s_{N N}}=5.02 \mathrm{TeV}$, no LHC (Large Hadron Collider, em inglês).

Estudar os quarks pesados formados durante as colisões relativísticas entre íons pesados é uma importante maneira para estudar o meio formado, conhecido como QGP (Quark Gluon Plasma, em inlgês). Isso se deve ao fato de que eles são criados no início da colisão e, portanto, interagem com o QGP durante toda sua existência. Em seguida, tais quarks fragmentam em mésons que podem decair, via canal semi-leptônico, em elétrons (e pósitrons). Esses léptons podem ser utilizados para indicar a existência de um quark pesado e permitir o estudo do QGP.

Os processos de espalhamento duro, fragmentação e decaimento dão origem a diversas partículas que estão colimadas em uma região espacial, a que chamam 'jato'. A reconstrução e o estudo de jatos têm sido usados para extrair mais informações do meio.

Os elétrons foram selecionados pelo TPC (Time Projection Chamber, em inglês) e pelo EMCal (Electromagnetic Calorimeter, em inglês). O método de massa invariante foi utilizado para excluir elétrons não provenientes do decaimento de quarks pesados. Os jatos foram reconstruídos com o auxílio das bibliotecas do Fastjet, algorítmo anti $-k T$ e $R=0.4$. A seleção de pares HFe-jato pode permitir uma melhor compreensão das propriedades do QGP e de sua interação com quarks pesados.

Foram obtidos, para pp e p-Pb, os espectros de momento $p_{\mathrm{T}, \text { chjet }}$ do jato, em dois intervalos de distância angular $\Delta \varphi$ entre o elétron e o jato: regiões em oposição (away) e colinear (near). Também foi obtida a distribuição da distância angular $\Delta \varphi$ para diferentes intervalos de momento $p_{\mathrm{T}}^{e}$ do elétron.

Os resultados para pp e p- $\mathrm{Pb}$ foram comparados através de dois observáveis: um em função do momento $p_{\mathrm{T} \text {,chjet }}$ do jato, e outro em função do momento $p_{\mathrm{T}}^{e}$ do elétron. O primeiro consistiu na razão dos espectros de momento $p_{\text {T,chjet }}$ do jato em pp e em p-Pb. O segundo, na razão do valores obtidos em pp e em p-Pb das áreas dos picos de cada uma das duas regiões de $\Delta \varphi$. Em ambos os casos, os valores das razões são compatíveis com a unidade, o que indica que a inexistência de efeitos extras em p-Pb em relação a pp. 



\section{Abstract}

This thesis presents the first measurement of the distribution of jets that are correlated to heavy-flavour decay electron (HFe), reconstructed and identified with ALICE (A Large Ion Collider Experiment). The observables were measured in pp collisions at center of mass energy $\sqrt{s}=8 \mathrm{TeV}$ and in $\mathrm{p}-\mathrm{Pb}$ at $\sqrt{s_{N N}}=5.02 \mathrm{TeV}$ at the Large Hadron Collider (LHC).

Exploring the heavy quarks created in relativistic heavy ion collisions is a powerful approach to study the new formed medium, known as Quark Gluon Plasma (QGP). This is due to the fact that they are created in the beginning of the collision, via hard scattering, and interact with the QGP throughout its whole existence. These quarks fragment into mesons that can decay (via the semi-electronic channel) into electrons (and positrons). These leptons can be used to identify the creation of a heavy quarks and allow QGP studies.

The hard scattering, fragmenting and decaying processes originate several particles that are collimated in a conical region, and they can be grouped in what is called 'jet'. The reconstruction and study of jets has been used to retrieve more information about the QGP and it is also a useful probe.

The selected electrons were identified by the Time Projection Chamber (TPC) and the Electromagnetic Calorimeter (EMCal). Heavy-flavour decay electrons were selected via invariant mass method. The jets were reconstructed by the Fastjet framework, with the algorithm anti $-k T$ and $R=0.4$. The HFe-jet pairs selection may allow a better comprehension of the QGP properties and its interactions with the heavy quarks.

A jet $p_{\mathrm{T}, \text { chjet }}$ spectrum was obtained, for $\mathrm{pp}$ and $\mathrm{p}-\mathrm{Pb}$, in two intervals of angular distance $\Delta \varphi$ between the jet and the electron: away and near sides. The angular distance $\Delta \varphi$ distribution was also obtained in different electron $p_{\mathrm{T}}^{e}$ intervals.

The results for $\mathrm{pp}$ and $\mathrm{p}-\mathrm{Pb}$ were compared through two observables: the first one as a function of the jet $p_{\mathrm{T}, \mathrm{chjet}}$, and the second, as a function of electron $p_{\mathrm{T}}^{e}$. The former consists in calculating the ratio of the jet $p_{\mathrm{T}, \text { chjet }}$ spectra, in $\mathrm{pp}$ and in $\mathrm{p}-\mathrm{Pb}$. The latter, consists in calculating the ratio of the areas, in $\mathrm{pp}$ and in $\mathrm{p}-\mathrm{Pb}$, of each peak in the $\Delta \varphi$ distribution. Both were compatible with the unity, which indicates that there is no extra effect in $\mathrm{p}-\mathrm{Pb}$ with respect to $\mathrm{pp}$. 



\section{List of Figures}

2.1 QCD running coupling . . . . . . . . . . . . . . . . . . . . . . 19

2.2 Hadron-muon ratio $R$ in electron-positron annihilation . . . . . . . . . . 20

2.3 Table of Standard Model particles . . . . . . . . . . . . . . . . . . 21

2.4 QCD phase diagram . . . . . . . . . . . . . . . . . . . . 22

2.5 QGP phase transition . . . . . . . . . . . . . . . . . . . . . 23

2.6 Time evolution in heavy-ion collisions . . . . . . . . . . . . . . . . . . 24

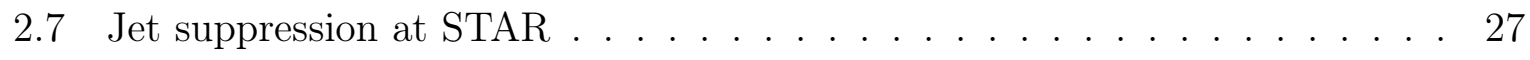

$2.8 R_{A A}$ measured at STAR $\ldots \ldots \ldots \ldots \ldots \ldots$

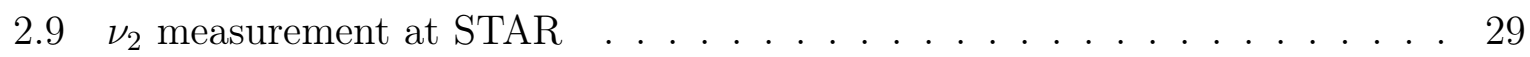

2.10 Charged particles $R_{p P b}$ at ALICE $\ldots \ldots \ldots \ldots$. . . . . . . . 30

2.11 Jets $R_{p P b}$ at ALICE . . . . . . . . . . . . . . . . 30

2.122 -D two particle correlation function at CMS $\ldots \ldots \ldots$. . . . . . . . 31

3.1 Heavy-flavour production Feynman diagrams . . . . . . . . . . . . . . . 34

3.2 Charm production processes comparison . . . . . . . . . . . . . . . 35

3.3 Charm fragmentation compilation from HERA . . . . . . . . . . . . . 35

3.4 Heavy-flavour decay electrons $\nu_{2}$ at ALICE . . . . . . . . . . . . . . . 37

3.5 Heavy-flavour decay electrons nuclear modification factor at ALICE . . . . 38

3.6 Collinear unsafe algorithms scheme . . . . . . . . . . . . . . . 40

3.7 Infrared unsafe algorithms scheme . . . . . . . . . . . . . . . . . . . 40

3.8 Pictoric jet reconstruction $\ldots \ldots \ldots \ldots$. . . . . . . . . . . . . . . . . . . . . 41

3.9 Jet Spectra for different $p_{\mathrm{T}}^{\text {trigger }}$ intervals $\ldots \ldots \ldots \ldots \ldots$. . . . . . . . . 45

3.10 STAR semi-inclusive jet spectrum . . . . . . . . . . . . . . . . . 45

3.11 Hadron-Jet $\Delta \varphi$ for different $p_{\mathrm{T}}^{\text {trigger }}$ ranges $\ldots \ldots \ldots \ldots \ldots$. . . . . . 46

3.12 Jet-hadron $\Delta \varphi$ at STAR $\ldots \ldots \ldots \ldots \ldots \ldots$. . . . . . . . . . . . . 47

3.13 Jet-hadron medium modification results observables at STAR . . . . . . . 47

4.1 Scheme of the ALICE detectors . . . . . . . . . . . . . . . . . . . . 49

4.2 LHC complex scheme . . . . . . . . . . . . . . . . . . . . . . 51

4.3 ITS Structure Scheme . . . . . . . . . . . . . . . . . . . . . . 52

4.4 TPC scheme . . . . . . . . . . . . . . . . . . . . . . . 53 
4.5 TPC energy $\operatorname{loss} . \ldots \ldots \ldots \ldots$

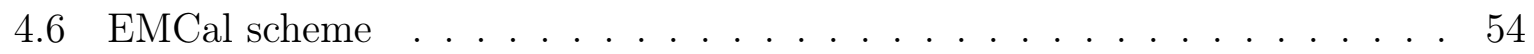

4.7 V0 Scheme . . . . . . . . . . . . . . . . . . . . . 55

$5.1 \quad \mathrm{~V} 0$ in MB trigger scheme $\ldots \ldots \ldots \ldots \ldots$

5.2 V0 times in MB trigger . . . . . . . . . . . . . . . . . . . . . . . . 58

$5.3 \quad$ EMCal trigger patches for different triggers . . . . . . . . . . . . . . . . 59

5.4 Clusterizer V1 scheme . . . . . . . . . . . . . . . . . . . . . . 60

5.5 EMCal shower shape variables . . . . . . . . . . . . . . . . . . 62

5.6 Track helix reconstruction scheme . . . . . . . . . . . . . . . . . . . . . 64

5.7 TPC energy loss distribution $\left(N_{\sigma T P C}^{e}\right) \ldots \ldots \ldots 6$

$6.1 \quad$ Electron-jet angles scheme . . . . . . . . . . . . . . . . . . 70

6.2 Electron-jet inclusive selection (TPC + EMCal) $(\mathrm{pp}) \ldots$. . . . . . . . . 71

6.3 Away side Electron-jet $E / p$ hadronic contamination $(\mathrm{pp})$. . . . . . . . . 72

$6.4 \quad$ Near side Electron-jet $E / p$ hadronic contamination (pp) . . . . . . . . . . 73

6.5 Hadronic contamination percentages (pp) . . . . . . . . . . . 73

6.6 Invariant mass distribution $(\mathrm{pp})$. . . . . . . . . . . . . . . 74

$6.7 \quad$ Invariant mass method efficiency $(\mathrm{pp})$. . . . . . . . . . . . . . . 75

6.8 Heavy-flavour decay electron identification efficiency $(\mathrm{pp})$. . . . . . . . . . 75

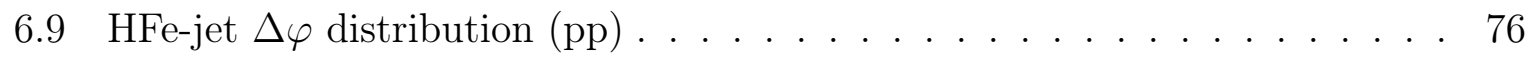

6.10 Response matrices $(\mathrm{pp})$. . . . . . . . . . . . . . . . . . . 77

6.11 Hadronic contamination for tracks only $(\mathrm{pp})$. . . . . . . . . . . . . . 79

6.12 Heavy-flavour electron $p_{\mathrm{T}}^{e}$ spectrum $(\mathrm{pp})$

6.13 Away side: HFe-jet normalized contributions and unfolded spectrum (pp) 80

6.14 Near side: HFe-jet normalized contributions and unfolded spectrum (pp) 80

6.15 Away side: HFe-jet spectrum with systematic uncertainties (pp) . . . . . 81

6.16 Near side: HFe-jet spectrum with systematic uncertainties (pp) . . . . . . 82

$7.1 \quad$ Electron-jet inclusive selection $(\mathrm{TPC}+\mathrm{EMCal})(\mathrm{p}-\mathrm{Pb}) \ldots$. . . . . . . . 86

$7.2 \quad$ Away side Electron-Jet $E / p$ hadronic contamination $(\mathrm{p}-\mathrm{Pb})]$. . . . . . . . 87

7.3 Near side Electron-Jet $E / p$ hadronic contamination $(\mathrm{p}-\mathrm{Pb})$. . . . . . . . 88

7.4 Hadronic contamination percentages $(\mathrm{p}-\mathrm{Pb}) \ldots \ldots$. . . . . . . . 88

7.5 Invariant mass distribution $(\mathrm{p}-\mathrm{Pb}) \ldots \ldots \ldots \ldots$. . . . . . . . . . 89

7.6 Invariant mass method efficiency $(\mathrm{p}-\mathrm{Pb}) \quad \ldots \ldots \ldots$. . . . . . . . . 89

7.7 Heavy-flavour decay electron identification efficiency $(\mathrm{p}-\mathrm{Pb})$. . . . . . . . . 90

7.8 HFe-jet $\Delta \varphi$ distribution $(\mathrm{p}-\mathrm{Pb}) \ldots \ldots \ldots \ldots \ldots$

7.9 Response matrices $(\mathrm{p}-\mathrm{Pb})$. . . . . . . . . . . . . . . . . . . . . 91

7.10 Hadronic contamination for tracks only $(\mathrm{p}-\mathrm{Pb})$. . . . . . . . . . . . . . 92

7.11 Heavy-flavour electron $p_{\mathrm{T}}^{e}$ spectrum $(\mathrm{p}-\mathrm{Pb}) \ldots \ldots$. . . . . . . . . . 92 
7.12 Away side: HFe-jet contributions and unfolded spectrum (p-Pb) . . . . . 93

7.13 Near side: HFe-jet contributions and unfolded spectrum (p-Pb) . . . . . . 93

7.14 Away side: HFe-jet spectrum with systematic uncertainties (p-Pb) . . . . 94

7.15 Near side: HFe-jet spectrum with systematic uncertainties (p-Pb) . . . . 94

8.1 FONLL scale factor $\ldots \ldots \ldots$. . . . . . . . . . . . . . . . . . 98

$8.2 \quad$ Away side $I_{p P b} \ldots \ldots \ldots \ldots \ldots \ldots \ldots$

$8.3 \quad$ Near side $I_{p P b} \ldots \ldots \ldots \ldots \ldots \ldots \ldots$

$8.4 \Delta \varphi$ distribution comparison between $\mathrm{pp}$ and $\mathrm{p}-\mathrm{Pb} \ldots \ldots$. . . . . . . 101

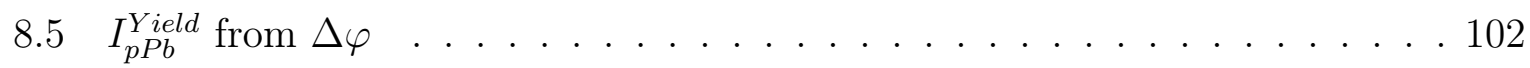

A.1 Geometric Variables . . . . . . . . . . . . . . . . . . . . 106

A.2 $N_{\sigma T P C}^{e}$ random rejection of hadrons . . . . . . . . . . . . . . 110

A.3 Middle Invariant mass distribution . . . . . . . . . . . . . . . . . . . . . . 111

A.4 Middle: HFe-jet normalized contributions and unfolded spectrum (pp) . . 112

A.5 Middle: HFe-jet normalized contributions and unfolded spectrum (p-Pb) 112

A.6 Away side Closure test spectrum (pp) . . . . . . . . . . . . . . . 116

A.7 Away side Closure test spectrum $(\mathrm{p}-\mathrm{Pb}) \ldots \ldots$. . . . . . . . . . . . 117

A.8 Near side Closure test spectrum $(\mathrm{pp})$. . . . . . . . . . . . . . . 117

A.9 Near side Closure test spectrum $(\mathrm{p}-\mathrm{Pb}) \ldots \ldots \ldots \ldots$. . . . . . . . 118

A.10 Unfolding $\Delta \mathcal{H}$ distribution $(\mathrm{pp})$

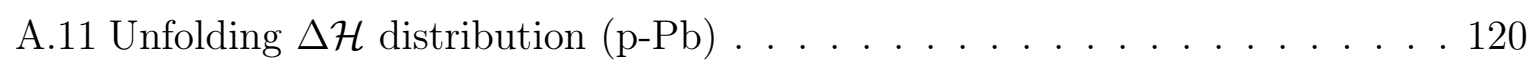

A.12 FONLL HFe cross section . . . . . . . . . . . . . . . . . . . . . 121

A.13 FONLL scale factor . . . . . . . . . . . . . . . . . . . . . 122

A.14 Away side: FONLL uncertainties . . . . . . . . . . . . . . . . . . . 122

A.15 Near side: FONLL uncertainties . . . . . . . . . . . . . . . . . . . . 123 



\section{Contents}

\begin{tabular}{lll}
\hline 1 & Introduction & 15
\end{tabular}

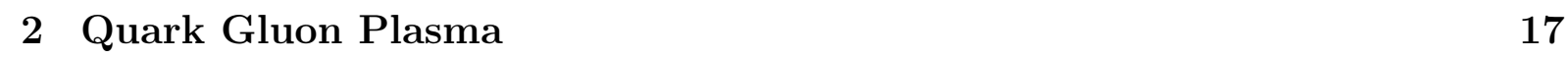

2.1 The Quantum Chromodynamics and the Standard Model . . . . . . . . . . 17

2.2 QCD in Heavy-Ion Collisions: The Quark-Gluon Plasma . . . . . . . . . . 21

2.3 Experimental Evidences of the QGP . . . . . . . . . . . . . . . . 25

2.4 QGP in Small Systems . . . . . . . . . . . . . . . . . . . . . . 29

3 Heavy Flavours and Jets 33

3.1 Heavy Flavour Production . . . . . . . . . . . . . . . . . . . . . . . 33

3.2 Heavy-flavour Measurements . . . . . . . . . . . . . . . . . . . . 37

3.3 Jets $\ldots \ldots \ldots \ldots \ldots$

3.4 Unfolding $\ldots \ldots \ldots \ldots \ldots$

3.5 Track-Jet Analysis . . . . . . . . . . . . . . . . . . . . . . . 43

\begin{tabular}{|lll}
4 & A Large Ion Collider Experiment & 49
\end{tabular}

4.1 Large Hadron Collider . . . . . . . . . . . . . . . . . . . . 50

4.2 Inner Tracking System . . . . . . . . . . . . . . . . . . . . . 51

4.3 Time Projection Chamber . . . . . . . . . . . . . . . . . . . 52

4.4 Electromagnetic Calorimeter . . . . . . . . . . . . . . . . . . . . 54

$4.5 \mathrm{V0} \ldots \ldots \ldots \ldots \ldots \ldots \ldots$

$\begin{array}{lll}5 \text { Data Analysis } & 57\end{array}$

5.1 Event Selection . . . . . . . . . . . . . . . . . . . . . 57

5.2 Cluster Reconstruction . . . . . . . . . . . . . . . . . . . . . 60

5.3 Track Reconstruction . . . . . . . . . . . . . . . . . . . . . . . . . . 62

5.4 Jet Reconstruction . . . . . . . . . . . . . . . . . . . . . . . 64

5.5 Electron Identification $\ldots \ldots \ldots \ldots$

6 Analysis in Proton-Proton Data $\quad 69$

6.1 Event Selection . . . . . . . . . . . . . . . . . . . . . . . . 69

6.2 Electron-Jet Pairs Selection . . . . . . . . . . . . . . . . . . . . . . . . . . 69 
6.3 Inclusive Electron-Jet Selection . . . . . . . . . . . . . . . . . . 70

6.4 Hadronic Contamination . . . . . . . . . . . . . . . . . . . . 71

6.5 Non-HF Electron Contamination . . . . . . . . . . . . . . . . . . . 74

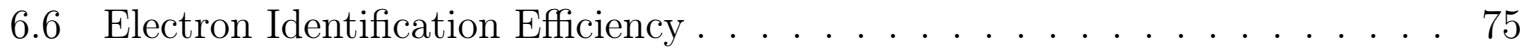

6.7 HF Electron-Jet $\Delta \varphi \ldots \ldots \ldots \ldots \ldots$

6.8 Unfolding . . . . . . . . . . . . . . . . . . . . . . . . . . . . 77

6.9 HF Electron-Jet Spectrum $\ldots \ldots \ldots \ldots$. . . . . . . . . . . . 78

6.10 Systematic Uncertainties . . . . . . . . . . . . . . . . . . . . 81

7 Analysis in Proton-Lead Data 85

7.1 Event Selection . . . . . . . . . . . . . . . . . . . . . . . . . . . 85

7.2 Electron-Jet Pairs Selection . . . . . . . . . . . . . . . . . 85

7.3 Inclusive Electron-Jet Selection . . . . . . . . . . . . . . . . . . 86

7.4 Hadronic Contamination . . . . . . . . . . . . . . . . . . . 86

7.5 Non-HF Electron Contamination … . . . . . . . . . . . . . . . . . 89

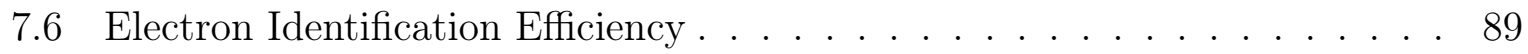

7.7 HF Electron-Jet $\Delta \varphi \ldots \ldots \ldots \ldots$

7.8 Unfolding . . . . . . . . . . . . . . . . . . . . . . . . . . . . . . . 91

7.9 HF Electron-Jet Spectrum . . . . . . . . . . . . . . . . . . . . . . . . . . . 91

7.10 Systematic Uncertainties . . . . . . . . . . . . . . . . . . . . . . . . 93

8 Discussion $\quad 97$

8.1 FONLL Energy Scaling . . . . . . . . . . . . . . . . . . . . . . . . . . . 97

$8.2 p_{\mathrm{T}}^{e}$-inclusive $I_{p P b} \ldots \ldots \ldots \ldots \ldots \ldots \ldots \ldots$

$8.3 \quad p_{\mathrm{T}, \mathrm{ch} \mathrm{jet} \text {-inclusive } I_{p P b}^{Y i e l d}} \ldots \ldots \ldots \ldots \ldots \ldots$

$\begin{array}{lll}9 & \text { Conclusions } & 103\end{array}$

\begin{tabular}{ll}
\hline A Appendix & 105
\end{tabular}

A.1 Kinematic Variables . . . . . . . . . . . . . . . . . . 105

A.2 Data Samples and Code in AliROOT . . . . . . . . . . . . . . 107

A.3 Random Rejection of $[-10,-3] N_{\sigma T P C}^{e}$ Region $\ldots . . . . . . . .110$

A.4 Middle Spectrum . . . . . . . . . . . . . . . . . . . . . . . . . . . . . 111

A.5 Systematic Uncertainties for $\Delta_{\text {side }} \ldots \ldots \ldots$. . . . . . . . . . . 113

A.6 Closure Test . . . . . . . . . . . . . . . . . . . . . . . . . . . . . . . . 116

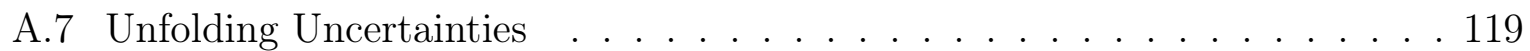

A.8 $\quad$ FONLL Scaling Factor $\ldots \ldots \ldots$. . . . . . . . . . . . . . . . . . 121

$\begin{array}{ll}\text { Glossary } & 125\end{array}$ 


\section{Introduction}

Ultrarelativistic heavy-ion collisions can produce a high temperature and/or a high energy density fireball. Quantum Chromodynamics (QCD) predicts that, in these extreme conditions, a new medium will be formed, the Quark-Gluon Plasma (QGP), in which quarks and gluons are deconfined from hadrons [1, 2]. The study of the QGP is one of the main goals of modern nuclear physics and the conditions for its formation can be attained in nuclei collisions at RHIC and LHC [3, 4, 5].

Heavy-flavour quarks, charm and bottom, are good probes to study the QGP because their masses are much larger than the masses of up, down and strange quarks, and, thus, are produced more probably in the early moments of the collisions [4]. Since the formation of the QGP is expected to happen after the heavy-flavour production, these quarks interact with the medium during its complete evolution. The heavy-flavour quarks can be studied through their fragmentation and decay products.

Nevertheless, measurements in heavy-ion collisions can have effects from the QGP itself and also from Cold Nuclear Matter (CNM), that are due to the structure of the colliding nuclei [3, 4]. Assessing the CNM effects is possible by studying nucleon-nucleon and nucleon-nucleus collisions. In the former, pertubative QCD (pQCD) can describe particle production, like heavy-flavour quarks and mesons. In the latter, however, the presence of a nucleus introduces CNM effects in the measurements and an extended QGP formation is not expected. Hence, a comparison between both systems measurements allows separating the pQCD, CNM and the QGP contributions.

Other probes that allow for the QGP studies are hard processes, i.e. interactions with high momentum transfer. They produce a collimated spray of particles that can be grouped in jets [6]. Some of the hard processes happen before the medium formation, like the heavy-flavour production, and are modified by interacting with it, and thus carry information about the medium properties. Experimentally, jets are defined by algorithms, and several results on jet modifications due to the medium were already presented [7].

In this thesis, electrons and positrons from heavy-flavour particles decay and jets are used to measure, for the first time, semi-inclusive, heavy-flavour related jet spectra and angular electron-jet (and positron-jet) correlation. They are obtained in pp collisions at $\sqrt{s}=8 \mathrm{TeV}$ and in $\mathrm{p}-\mathrm{Pb}$ collisions at $\sqrt{s_{N N}}=5.02 \mathrm{TeV}$ recorded by ALICE in 2012 and 2013, respectively. 
The electron and the positron are treated indistinctively in this analysis. Therefore, the term electron represents both electron and its anti-particle, and the term positron is, heafter, omitted, unless their contrast is needed.

The electron identification was performed using a combination of the Time Projection Chamber (TPC) and the Electromagnetic Calorimeter (EMCal). The events used in this analysis were triggered by the EMCal, that allowed the selection of electron tracks with $3<p_{\mathrm{T}}^{\text {track }}<18 \mathrm{GeV} / c$. The jets were reconstructed with resolution parameter $R=0.4$, using the Fastjet [8] framework, with $10<p_{T, \text { jet }}^{c h}<80 \mathrm{GeV} / c$, and their tracks were not required to have a matched cluster in the EMCal.

In the following chapters, one finds the theoretical introduction to heavy ion collisions, which includes brief introductions to QCD and the physics of the QGP (chapter 2). Chapter 3 describes heavy-flavour electron identification procedure and jet reconstruction. Chapter 4 shows the experimental setup and chapter 5 explains how the data sets are selected and recorded. Chapters 6 and 7 have the details of the analysis, chapter 8 discuss the results, and, finally, the conclusions are in chapter 9. 


\section{Quark Gluon Plasma}

In this chapter, the theoretical fundaments of the Standard Model, that can describe the physics of heavy-ion collisions, specially the Quantum Chromodynamics, will be discussed. The Quark-Gluon Plasma formation and its properties are discussed as is the heavy-flavour production in these conditions. Finally, some experimental results regarding the general motivation for heavy-ion colliders and analyses will be presented.

\subsection{The Quantum Chromodynamics and the Standard Model}

In the beginning of the 20th century, electron, proton and neutron were the only known subatomic particles. Their electromagnetic interaction was used to probe the atoms and nuclei structure. However, after particles accelerators reached higher energies, in the decade 1950, there were dozens of new particle species that were treated as "fundamental" [9. Solving this problem, also called "particle zoo" due to the great number of particles, had several attempts and, by the end of the 1950s, there were three classifications of particles: leptons, baryons and mesons.

The electron, the muon, the tau, the electron neutrino, the muon neutrino and the tau neutrino, as well as their anti-partners, are classified as leptons. The proton, the neutron and several other heavier particles, as well as their anti-particles, are baryons, whose number (baryons minus anti-baryons) is conserved in interaction processes. The pion and the kaon (today there are more examples) are mesons, which interact with baryons but do not have their number conserved. Mesons and baryons were grouped in a more general classification as hadrons.

In 1964, Gell-Man and Zweig, independently, approched on grouping the hadrons in what is called the Quark Model [10]. Their proposal was to consider a smaller set of fundamental particles, which were named quarks. Baryons would be a composition of three quarks, and mesons, one quark and one anti-quark. Each quark would come in three different flavours (Up, Down and Strange), have a baryon number of $1 / 3$, a electromagnetic charge of $-1 / 3$ or $2 / 3$, and a strangeness number of 0 or -1 . Table 2.1 shows a summary of quantum numbers associated to all proposed quarks at the time. 
Table 2.1: Quark Model Particles proposed by Gell-Man and Zweig in 1964

\begin{tabular}{cccc}
\hline \hline Quark & Baryon Number & Electromagnetic Charge & Strangeness Number \\
\hline Up & $1 / 3$ & $+2 / 3$ & 0 \\
Down & $1 / 3$ & $-1 / 3$ & 0 \\
Strange & $1 / 3$ & $-1 / 3$ & -1 \\
\hline \hline
\end{tabular}

In 1973, Fritz, Gell-Mann and Leutwyler proposed the Quantum Chromodynamics (QCD), a gauge field theory that describes the interaction between quarks [10, 9]. The mediators of this interaction were called gluons, which are massless, flavourless, and have zero electromagnetic charge. This description was associated to the SU(3) gauge symmetry group [10], whose eight generator matrices $\lambda_{i}$, the so called Gell-Mann matrices, were associated to gauge bosons (gluons), and its three eigenvectors, to the three possible colours of quarks $(r, g$ or $b$, a new quantum number). Baryons and mesons might be colourless (or white), i.e. have the same amount either of each colour or colours and their respective anti-colours. By the end of the decade 1980, the model had to be modified in order to include more particles, that were discovered after 1974. Then, there were six quarks, each having one out of three possible different colour-charges, and their respectives anti-particles.

The Lagrangian of the QCD can be expressed as in the equation 2.1

$$
\mathcal{L}_{Q C D}=\sum_{c, c^{\prime}, f} \bar{q}_{f}^{c} i \gamma^{\mu}\left(D_{\mu}\right)_{c, c^{\prime}} q_{f}^{c^{\prime}}-m_{f} \bar{q}_{f}^{c^{\prime}} q_{f}^{c^{\prime}}-\frac{1}{4} G^{c, \mu \nu} G_{\mu, \nu}^{c}
$$

where $\left(D_{\mu}\right)_{c, c^{\prime}} q_{f}^{c^{\prime}} \equiv\left[\partial_{\mu}-i g_{s} \frac{\lambda_{c, c^{\prime}}^{a}}{2} A_{a}^{\mu}\right] q_{f}^{c^{\prime}}, q$ are the quark fields, with their colour indices $c, c^{\prime}$, the flavour index $f$ and their mass $m_{f}$. The $G^{c, \mu \nu}$ are the gluon fields. The $\mu, \nu$ are the Lorentz indices.

$$
\left(D_{\mu}\right)_{c, c^{\prime}} q_{f}^{c^{\prime}} \equiv\left[\partial_{\mu}-i g_{s} \frac{\lambda_{c, c^{\prime}}^{a}}{2} A_{a}^{\mu}\right] q_{f}^{c^{\prime}}
$$

After renormalizing [10] the QCD, one finds that the coupling constant depends on the exchanged momentum $Q^{2}$ between the interacting particles.. It is called running coupling $\alpha_{s}$ and it is described by equation 2.2 .

$$
\alpha_{s}\left(Q^{2}\right)=\frac{\alpha_{s}\left(\mu^{2}\right)}{1+\alpha_{s}\left(\mu^{2}\right) \frac{11 N_{c}-2 N_{f}}{12 \pi} \ln \left(\frac{Q^{2}}{\mu^{2}}\right)}
$$

A renormalization parameter $\mu$ is introduced in its definition, and, since the formula depends on pertubation theory, any value is valid for $\mu$ as long as the resulting coupling stays below the unity. The other variables are the exchanged four-momentum $Q^{2}$, the number of colours $N_{c}$, and the number of quark flavours $N_{f}$. 
By introducing a new variable $\Lambda_{Q C D}$ defined as shown in equation 2.3

$$
\ln \left(\Lambda_{Q C D}^{2}\right)=\ln \left(\mu^{2}\right)-\frac{12 \pi}{\alpha_{s}(\mu)\left[11 N_{c}-2 N_{f}\right]}
$$

the running coupling can be rewritten as in equation 2.4, from which one of the most important results of the QCD can be seen. For higher energies, i.e. $Q^{2} \gg \Lambda_{Q C D}$, the coupling becomes weak and the quarks can be treated as free particles, what is called asymptotic freedom [10]. On the other hand, the coupling becomes larger when the energy scale decreases (or the distance increases). For instance, inside a meson, the interaction between the quark-anti-quark pair would become stronger and make it energetically more favorable to produce another pair of quark and anti-quark, as they go away from each other [10]. The newly produced quarks are combined into mesons. Quarks have never been observed free and this is called (colour) confinement.

The values of $\alpha_{s}$ calculated from measurements and pertubative QCD (pQCD), shown in figure 2.1 (from [11]), demonstrates the predictions and, in particular, the asymptotic freedom behaviour. The values for $\Lambda_{Q C D}$ are between $100 \mathrm{MeV}$ and $500 \mathrm{MeV}$.

$$
\alpha_{s}\left(Q^{2}\right)=\frac{12 \pi}{\left[11 N_{c}-2 N_{f}\right] \ln \left(\frac{Q^{2}}{\Lambda_{Q C D}^{2}}\right)}
$$

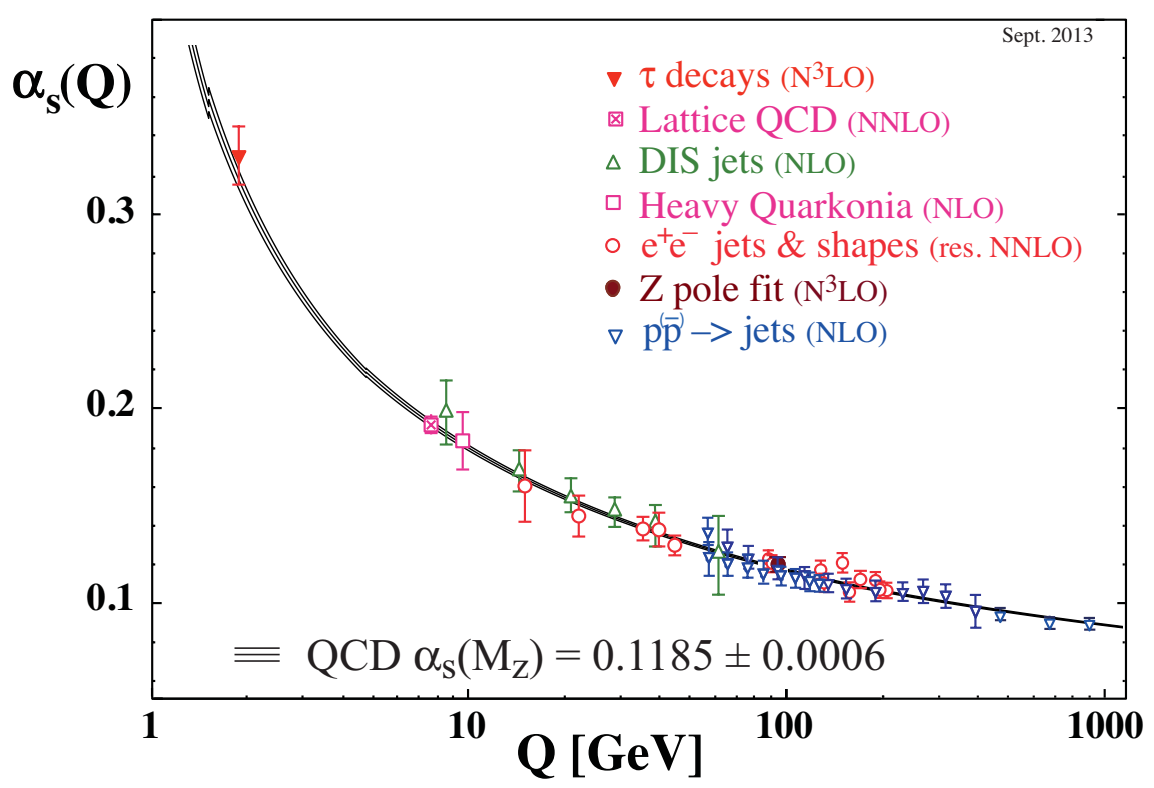

Figure 2.1: Summary of the measurements of $\alpha_{s}$ as a function of the energy scale $Q$ [11]. The respective degree of QCD perturbation theory used in the extraction of $\alpha_{s}$ is indicated in brackets (NLO: next-to-leading order; NNLO: next-to-next-to leading order; res. NNLO: NNLO matched with resummed next-to-leading logs; N3LO: next-to-NNLO). The mass of the Z boson $M_{Z}$ is used to fix the $\Lambda_{Q C D}$ scale. 
The electromagnetic interaction, i.e. the interaction between particles that carry electromagnetic charge, is described by the Quantum Electrodynamics (QED). The QED is a gauge theory associated to the gauge group of symmetries $\mathrm{U}(1)$, and the photon is the gauge boson that mediates it. Similarly, the weak interaction between all the twelve leptons and quarks is associated to the gauge symmetry group $\mathrm{SU}(2)$, and it is mediated by three gauge bosons: $Z$-boson, and $W^{ \pm}$. Both QED and Weak interactions are unified in the Electroweak theory.

From the QED and the QCD calculations, one finds that the ratio between the hadron and the $\mu^{+} \mu^{-}$production $R$ is proportional to the electronic charges of the different flavours (equation 2.5. So the values of $R$ as a function of the energy of the center of mass of the $e^{+} e^{-}$collision show (in figure 2.2 [10, 12]) that the number of flavours changes when certain thresholds are passed, i.e. when the energy is sufficient to produce quarks of a new flavour with a greater mass, what is an evidence of quark's flavours and colours.

$$
R \equiv \frac{\sigma\left(e^{+} e^{-} \rightarrow \text { hadrons }\right)}{\sigma\left(e^{+} e^{-} \rightarrow \mu^{+} \mu^{-}\right)}=N_{\text {colours }} \times \sum_{\text {flavours }} e_{\text {quark }}^{2}
$$

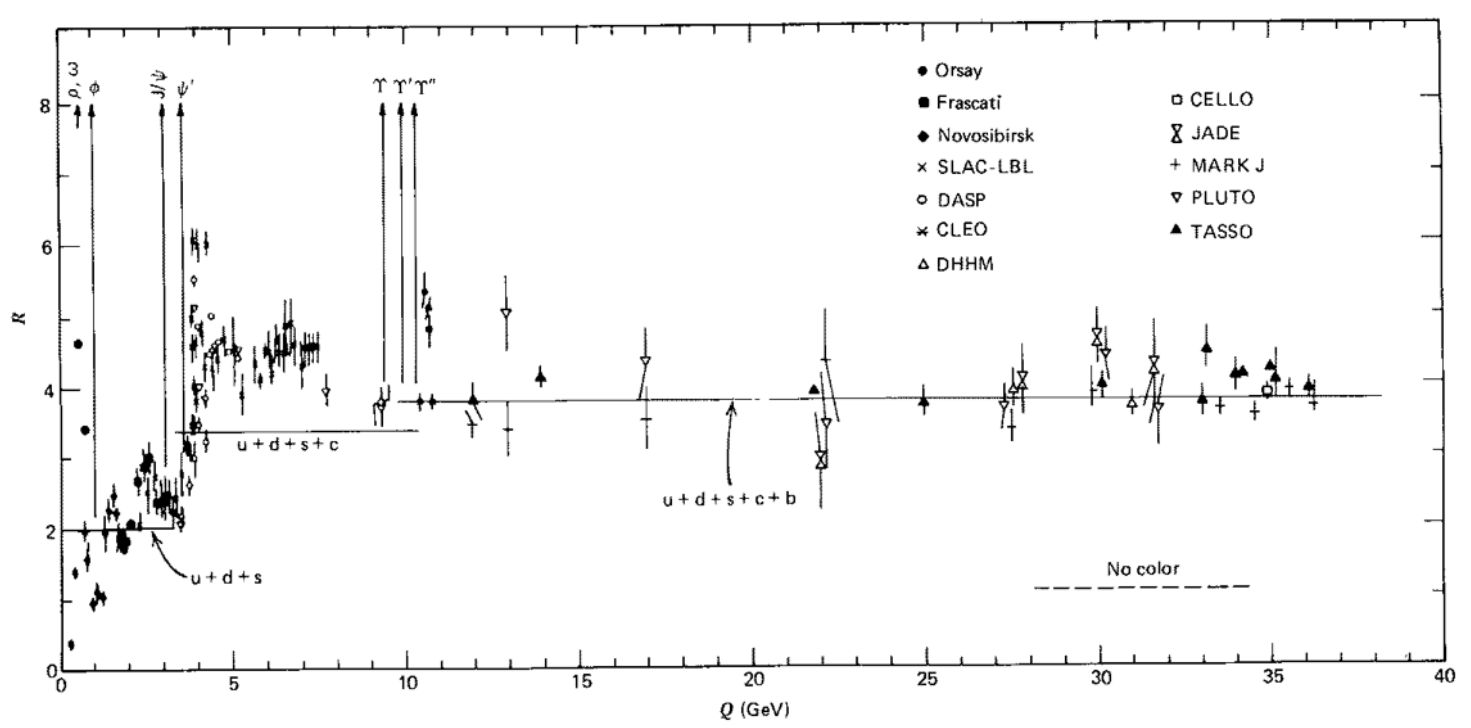

Figure 2.2: Hadron-muon pairs cross section ratio $R$ in electron-positron annihilation [10]. There are some visible regions, separated in steps, that are related to a new quark mass threshold being overpassed, as expected from equation 2.5 .

In the current view, the QCD, QED and the Weak Interactions compose the Standard Model (3 colours, 6 quarks, 6 leptons, their anti-particles, and 12 gauge bosons). Additionaly, it is also necessary an extra mechanism to explain the origin of the electroweak symmetry breaking and the non-zero mass of its gauge bosons. The Higgs mechanism does so by introducing a fundamental scalar field to the Standard Model that is related to the Higgs boson. It also gives rise to the mass of some fundamental particles through the Yukawa interaction. Figure 2.3 [13] summarizes schematically the particles and the 
interaction carriers.

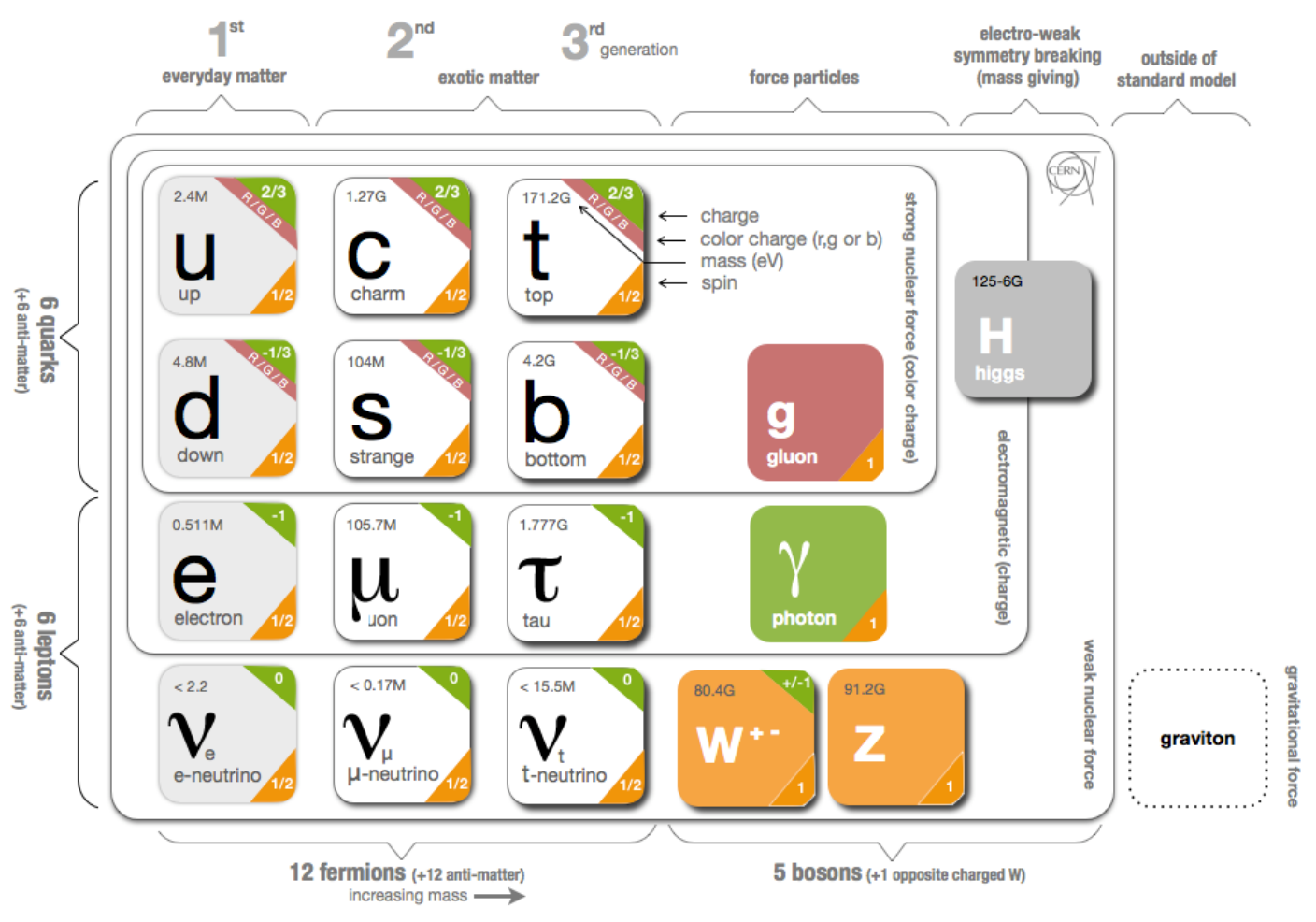

Figure 2.3: Schematic table of Standard Model particles [13]: the quarks are in the top, left part; leptons are in the bottom, left part; the interaction carrier gauge bosons are on the right; The first three lefthand columns represent the fermions' generations. The particles that interact are inside the same rectangle.

\subsection{QCD in Heavy-Ion Collisions: The Quark-Gluon Plasma}

As stated in the previous section, the QCD predicts a special behaviour for high energies, or short distances, that is known as asymptotic freedom [2, 14]. This means that, during the first stages of the universe evolution, when its temperatures are expected to be extremely high, and in the core of neutron stars, where the particle density would be high enough, quarks and gluons would be in a deconfined state, known as Quark-Gluon Plasma (QGP) [3, 4].

Figure 2.4 shows a theoretical QCD phase diagram for the temperature and the baryonic chemical potential [15]. For low temperatures and baryonic chemical potentials, the matter is expected to exist confined in hadrons, i.e. quarks and gluons are inside protons and neutrons. When these quantities exceed some threshold, a phase transition might occur so quarks and gluons are freed from the nucleon and become the QGP. 
Since neutron stars and the early universe are not accessible, the extreme conditions to explore the QGP can be achieved in high-energy nuclei collisions. The Relativistic HeavyIon Collider (RHIC) and the Large Hadron Collider (LHC) have been used to create the conditions where the QGP can be studied.

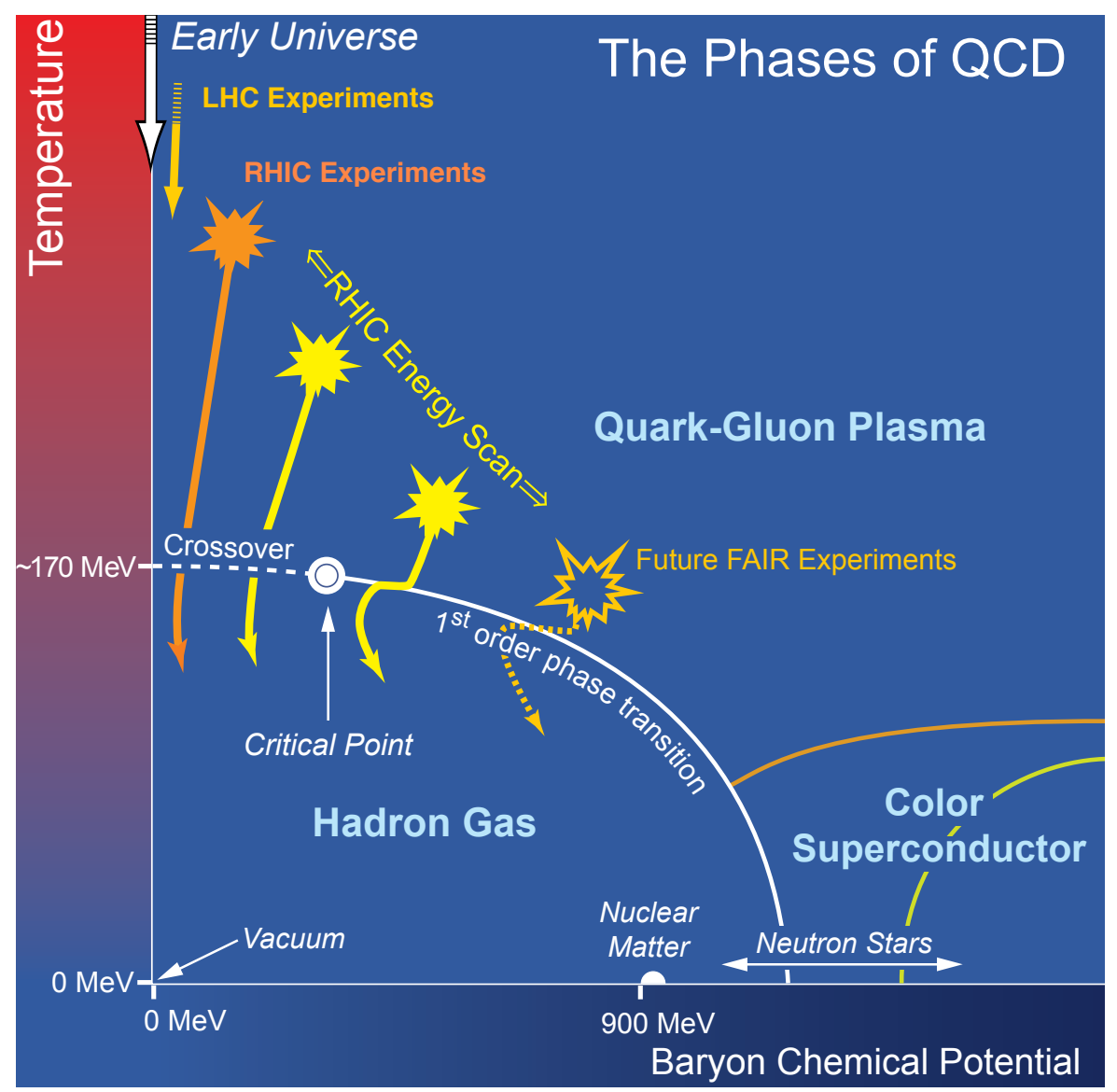

Figure 2.4: The QCD phase diagram (adapted from [15]) has: a hadronic state, where there are low temperatures and low baryonic chemical potential (leftmost, bottommost region); the QGP region, where there are high temperatures and baryonic potential (top region); and a neutron star region, where the baryonic potential is high and the temperatures are low (rightmost, bottommost region). The figure shows the state or evolution of the systems created in experiments (e.g. RHIC produces a system in high temperatures that evolves back to a hadronic state - orange arrow).

The phase diagram can be studied through an equation of state (EoS) that describes the relation between the temperature $T$ and the baryonic chemical potential. The EoS for an ideal gas of masseless quarks and gluons [3, 4, 16] (equation 2.6) relates the energy density $\epsilon_{S B}$ (and the pressure $p_{S B}$ ) to the degrees of freedom of the system, i.e. the number of colours $N_{c}$ and the number of flavours $N_{f}$, as does the massless pion gas equation of state [16] (equation 2.7, where $N_{\pi}=3$, for $\pi^{0}$ and $\pi^{ \pm}$).

$$
\begin{gathered}
\frac{\epsilon_{S B}}{3 T^{4}}=\frac{p_{S B}}{T^{4}}=\frac{\pi^{2}}{90}\left[2\left(N_{c}^{2}-1\right)+\frac{7}{2} N_{c} N_{f}\right] \\
\frac{\epsilon_{S B}}{3 T^{3}}=\frac{p_{S B}}{T^{4}}=\frac{\pi^{2}}{90} N_{\pi}
\end{gathered}
$$


Both EoS are extremely simplified and their absolute values are not comparable to the reality. Lattice QCD (1QCD) calculations show a transition that links them, and a transition temperature of $\approx 170 \mathrm{MeV}$ [17], for zero baryon density. This is shown in figure 2.4. where one finds the distribution of energy density-temperature ratio $\frac{\epsilon}{T^{4}}$ as a function of the temperature divided by the transition temperature $\frac{T}{T_{c}}$. It has three different behaviours that depend on the temperature values:

- $T<T_{c}$ : In this region, the system would behave as a hadron gas, or similar to the massless pion ideal gas (equation 2.7).

- $T \approx T_{c}$ : In this region, the system passes through the phase transition from the hadronic gas to the QGP.

- $T>T_{c}$ : In this region, the system would behave as the QGP, or similar to the massless quarks and gluons ideal gas (equation 2.6.

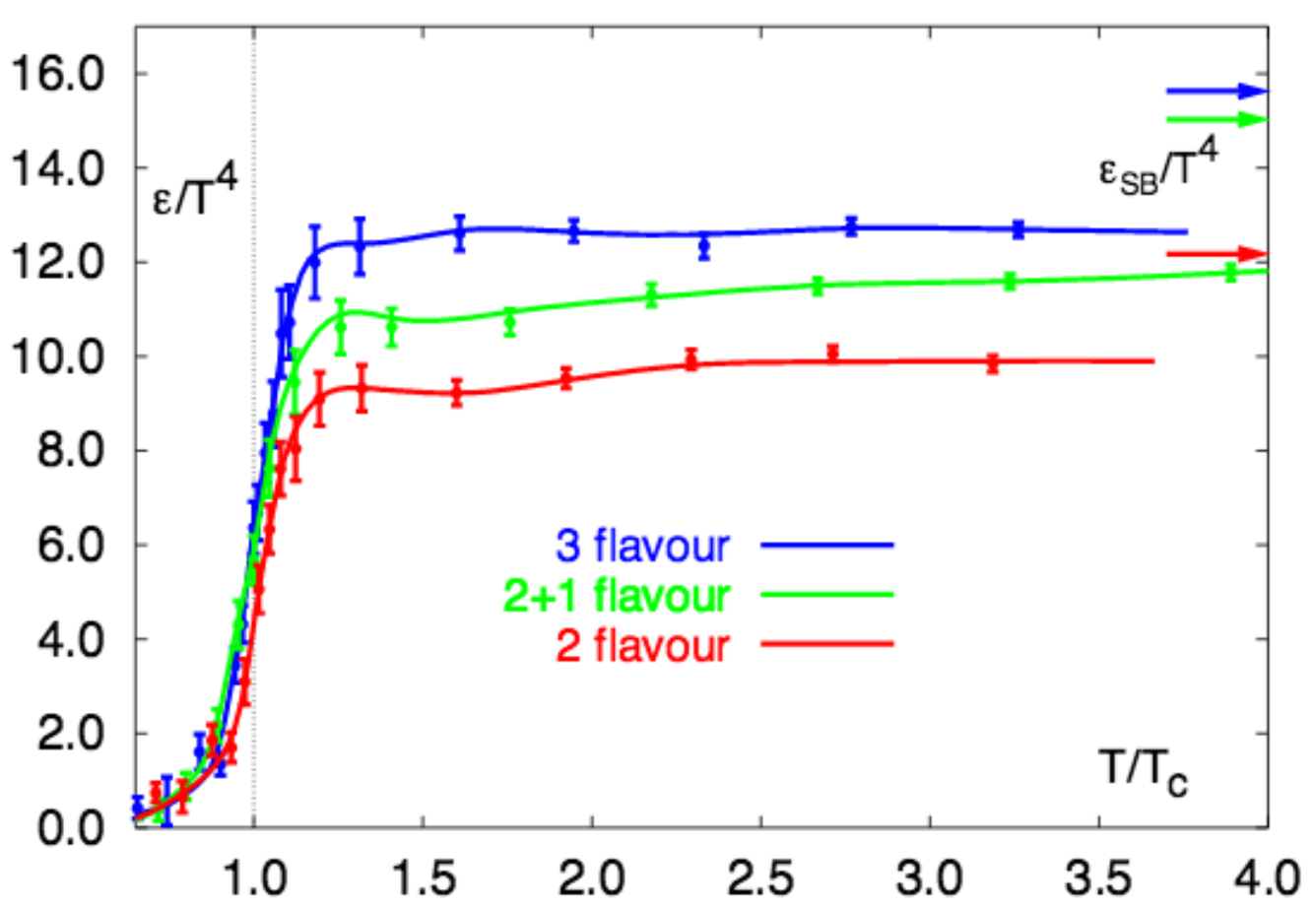

Figure 2.5: QGP phase transition as calculated in [18]. The different colours mean different number of flavours (degrees of freedom) and the arrows on the top right mark the values from the ideal, Stefan-Boltzmann gas calculations. Although the absolute values do not agree, they respect the hierarchy.

The predicted transition links, qualitatively, the perfect Stefan-Boltzman gas of pions $\left(\frac{\epsilon_{S B}}{T^{4}} \approx 1\right)$ to the perfect Stefan-Boltzman gas of quarks and gluons $\left(\frac{\epsilon_{S B}}{T^{4}}\right.$ values are indicated by arrows in figure 2.5). In the following section, the space-time evolution of the relativistic heavy ion collisions will be discussed. 


\section{Probing the QGP: Heavy-Ion Collisions}

The only way humans found to reproduce the necessary conditions to QGP formation is colliding particles at relativistic energies. In a certain way, these collisions mimic the early universe matter and allows assessing a high temperature and energy density system in the controlled environment of the laboratory. In figure 2.6 [19], one finds the standard model of the space-time evolution of a heavy ion collision, which can be divided in the following phases:

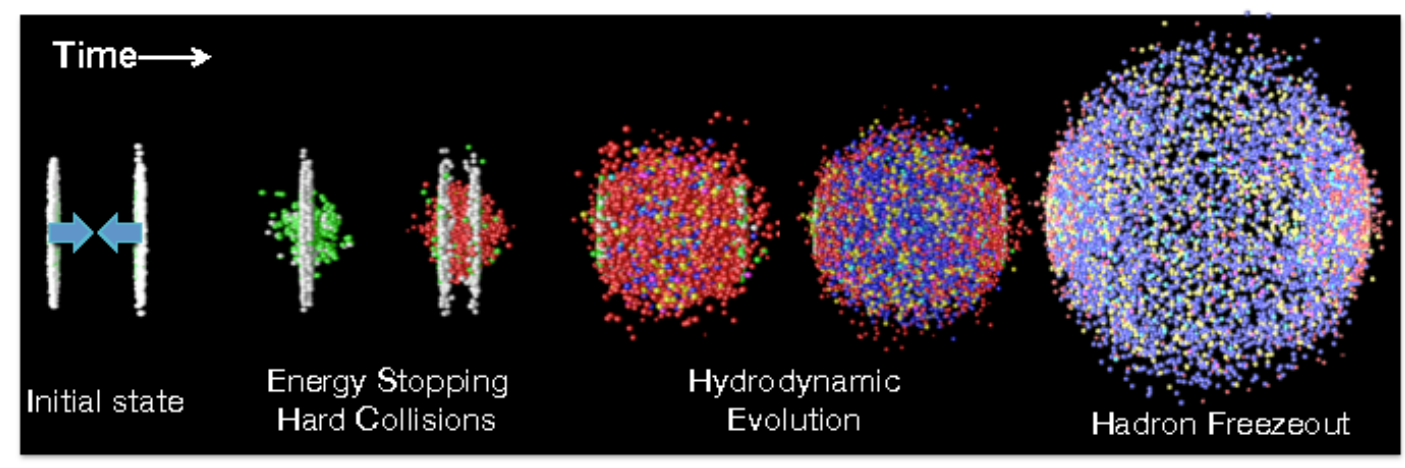

Figure 2.6: The time evolution in heavy ion collisions goes from an initial state (not interacting nuclei in relativistic velocities), passes through collisions (hard scatterings) that forms a medium, and evolves hydrodynamicaly until its freeze-out, when particles stop interacting [19].

- Initial State: Before the collision, the two nuclei are in ultra-relativistic speed, Lorentz-contracted, going in opposite directions. When they collide, hundreds of particles (quarks and gluons) are produced in a small volume and they interact in the asymptotic freedom regime;

- Pre-equilibrium: Just after the collision, the produced particles interact with each other without regrouping in baryons or mesons. At this stage, the increasing density favours the creation of more quark-antiquark pairs $(q \bar{q})$;

- Formation of the QGP: When the matter reaches a (local) equilibrium, the particles behave like a fluid, in what is called QGP state. Hydrodynamics models can be used to explain this stage;

- Hadronization: The gradient of the pressure inside the QGP causes an expansion of the medium, which decreases the (energy and particle) density. In these conditions, the quarks and gluons are confined in hadrons and mesons again (Chemical Freezeout);

- Kinetic Freeze-out: The hadron density continues to decrease until their elastic collisions cease and they fly through the detectors. At this moment, their momenta are fixed until interacting with the detectors and electromagnetic fields. 
Heavy-ion collisions is one way of bringing the matter to the QGP state (top left part of figure 2.4 , and its evolution in time would be the way back to the ordinary conditions (bottom left part of the figure 2.4). In terms of temperature, the matter is put in the right-hand region of the figure 2.5 and brought back to the left-hand region, passing through the transition temperature $T_{c}$, when the chemical freeze-out occurs.

After the freeze-out, the particles no longer interact and the medium is not the QGP, but it is, instead, a perfect-like gas made of hadrons. Hydrodynamics cannot be used and other models [20, 21, 14] can describe the system, assuming the momentum distribution is the same in both the QGP and the hadron gas.

\subsection{Experimental Evidences of the QGP}

After decades of data taking, some evidences of the QGP formation were acquired. In this section, some of them are summarized so one can have an overview of the well stablished ones. Namely they are: the energy density achieved in experiments; the indications of thermalization of the medium; the particle suppression; and the azimutal anisotropy dependence on $p_{T}$.

\section{Energy Density}

According to Bjorken [16], the transverse energy density of the medium can be estimated by using the transverse energy distribution, the time of formation of the QGP and the overlap area of the nuclei. He considers the following hypotheses:

- The medium is formed in a really thin region $d z$ in the longitudinal axis;

- The time of formation of the medium is $\tau_{f}$;

- The maximum longitudinal speed is $\frac{d z}{\tau_{f}}$, i.e. particles cannot escape the medium region before its complete formation at $\tau_{f}$;

- The number of created particles is $d N=\left(\frac{d z}{\tau_{f}}\right) \frac{d N}{d \beta_{\|}}$. Since the change in $z$ is so small that the particle production is uniform, the total production, for all $\beta_{\|}$velocities, is simply their product;

- For $y=\beta_{\|}=0$, the differentials are equal: $\frac{d N}{d \beta_{\|}}=\frac{d N}{d y}$; 
The energy density would be the ratio between the number of produced particles, times their mean transverse energy (their longitudinal energies are negligible) divided by the volume ( $d z$ times the overlap area $A_{o}$ ) of the medium. Equation 2.8 uses these hypotheses in order to obtain a simple relation.

$$
\begin{aligned}
\left\langle\epsilon\left(\tau_{f}\right)\right\rangle & =\frac{d N\left(\tau_{f}\right)}{d z} \frac{\left\langle m_{T}\right\rangle}{A_{o}} \\
& =\frac{d E_{T}\left(\tau_{f}\right)}{d y} \frac{1}{\tau_{f} A_{o}}
\end{aligned}
$$

PHENIX measurements [4] for $\frac{d N}{d \eta}, \frac{d E_{T}}{d \eta}$ and $\left\langle m_{T}\right\rangle$ are used to obtain an energy density estimates of at least $5 \mathrm{GeV} / \mathrm{fm}^{3}$. Lattice QCD calculations predicts a hadron-to-QGP transition energy $\epsilon \approx 1 \mathrm{GeV} / \mathrm{fm}^{3}$, which means that the formation of the QGP is expected to happen in RHIC and LHC conditions.

\section{Dihadron Azimuthal Correlations}

Figure 2.7 [3] shows the distribution of the azimuthal angular difference between high$p_{\mathrm{T}}$ hadrons, in $\mathrm{pp}, \mathrm{d}-\mathrm{Au}$ and $\mathrm{Au}-\mathrm{Au}$ collisions. For a hadron trigger with $p_{\mathrm{T}}^{\text {trigger }}>4$ $\mathrm{GeV} / \mathrm{c}$, one expects a peak at $\Delta \varphi \approx 0$ (near side) that relates to all the particles produced by the same hard process that originated the trigger particle. Thus, by the conservation of momentum, a peak in the opposite direction of the hight- $p_{\mathrm{T}}$ hadron, at $\Delta \varphi \approx \pi$ (away side), is also expected. This scenario is observed for pp and d-Au data, but for Au-Au the distribution is rather flat in the away side. This could be explained by the original away side jet of particles interacting with a high density medium and it is considered as another evidence of the QGP existence.

\section{$R_{A A}$ and Particle Suppression}

The charged hadron spectra measured at RHIC exhibit a (decreasing) power law behaviour with increasing $p_{\mathrm{T}}$, which is related to hard scattering process in QCD. Therefore, these can be used to evaluate the hadronic interaction with the medium through the comparison of the measurements performed with different systems, namely pp, d-Au and $\mathrm{Au}-\mathrm{Au}$ (or $\mathrm{p}-\mathrm{Pb}$ and $\mathrm{Pb}-\mathrm{Pb}$ ).

The charged hadron spectrum of the $\mathrm{AB}$ system, divided by the one from $\mathrm{pp}$ and normalized by the number of binnary collisions $N_{\text {Coll }}$, i.e. the number of collisions between two nucleons, is called Nuclear Modification Factor, $R_{A B}$ (equation 2.9, where $\mathrm{AB}$ can be either d-Au or $\mathrm{Au}-\mathrm{Au}$ - $\mathrm{p}-\mathrm{Pb}$ or $\mathrm{Pb}-\mathrm{Pb})$ :

$$
R_{\mathrm{AB}}=\frac{1}{N_{\text {Coll }}} \frac{d \mathrm{~N}^{\mathrm{AB}} / d p_{\mathrm{T}}}{d \mathrm{~N}^{p p} / d p_{\mathrm{T}}}
$$




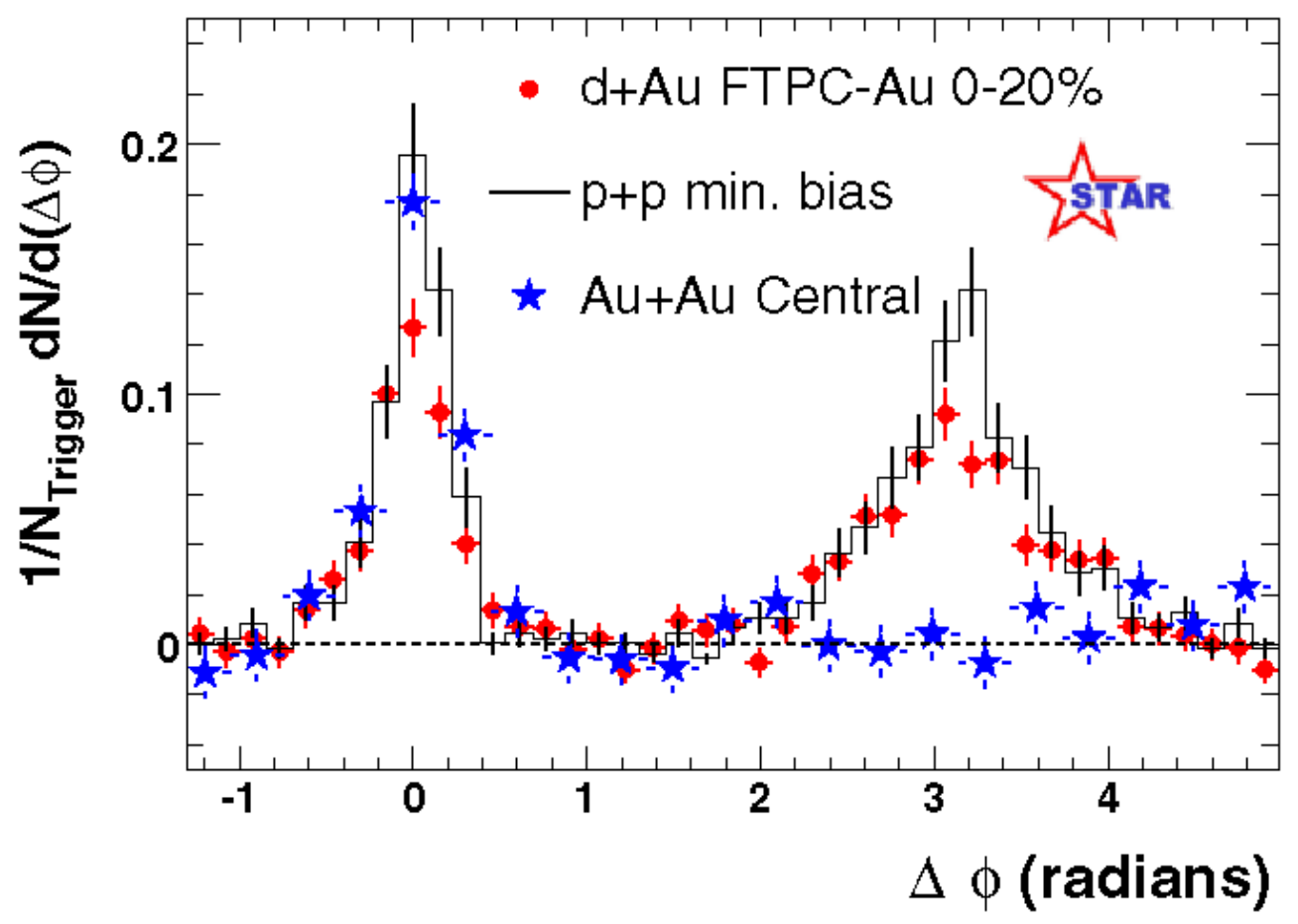

Figure 2.7: The angular correlation between hadrons, measured at STAR [3], shows that, in comparison with pp and $\mathrm{d}-\mathrm{Au}$, the $\mathrm{Au}-\mathrm{Au}$ result shows a Jet Suppression (quenching) in the away side region $(\Delta \varphi \approx \pi)$.

where $\frac{d N}{d p_{\mathrm{T}}}$ is the particle $p_{\mathrm{T}}$ spectrum; the $\mathrm{AB}$ and $p p$ indicate the collision system; and $N_{C o l l}$ is the number of (binary) nucleon-nucleon collisions.

Figure 2.8 3] shows these distributions obtained by the STAR collaboration, where one sees that, for $\mathrm{Au}-\mathrm{Au}$ central collisions (i.e. Au-Au with a greater number of participant nucleons), the hadrons are produced in a smaller quantity with respect to pp collisions. For $\mathrm{d}$-Au collisions, the hadronic production is enhanced for a large $p_{\mathrm{T}}$ range. The effect on $\mathrm{d}-\mathrm{Au}$ data is generaly atributed to the influence of multiple parton scattering through the cold nuclear matter (CNM, discussed in section 2.4) prior to the hard processes that generates the high- $p_{T}$ hadrons. Thus the suppression observed in Au-Au data must not be due to initial state nuclear effects, but due to interactions with the dense medium formed in these collisions.

\section{Azimutal Anisotropy}

When two nuclei collide, the overlap region for non-central collisions has approximately an ellipsoidal shape that results in the same spatial distribution for the produced particles. While the spatial distribution is anisotropic, the transverse momentum distribution must be isotropic in the initial stages. Therefore, when the QGP is created, the gradient of pressure is larger along the impact parameter direction than in the perpendicular 


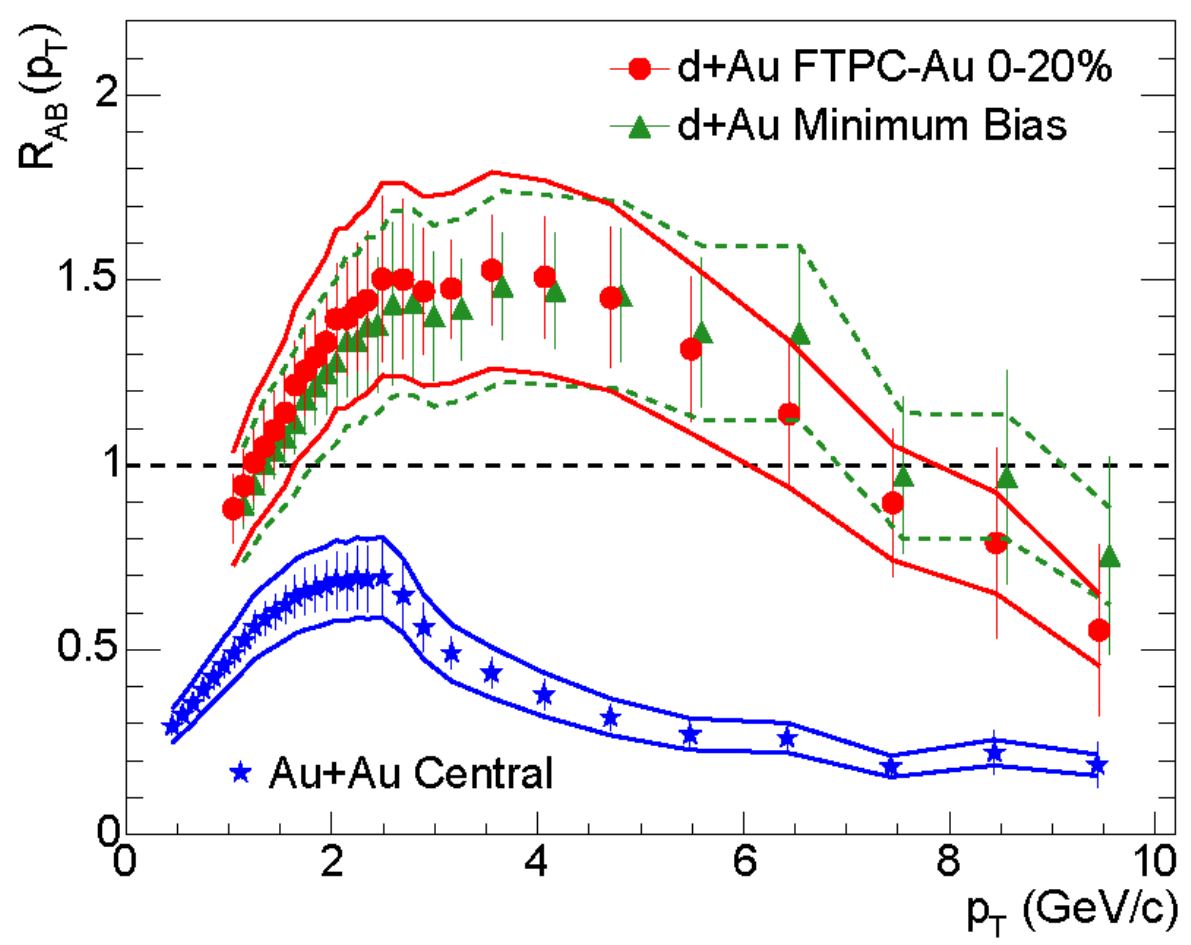

Figure 2.8: Hadrons and $\pi^{0} \mathrm{~S} R_{A A}$ measured at STAR [3] show a modification of the $p_{\mathrm{T}}$ distribution. They are due to CNM and QGP effects.

direction. As the medium expands, the momentum distribution becomes anisotropic too: along the impact parameter direction, the transverse momenta increase more than in the perpendicular direction due to the presence of more medium in this direction [4].

The angular distribution of particles can be decomposed in a Fourrier series and truncated in the second term, equation 2.10 ,

$$
\frac{d \mathrm{~N}}{d p_{\mathrm{T}} d \varphi}\left(p_{\mathrm{T}}\right)=\mathrm{N}_{0}\left[1+2 v_{2}\left(p_{\mathrm{T}}\right) \cos (2 \varphi)\right]
$$

where $N_{0}$ is a normalization constant, $\varphi$ is the angle and $\nu_{2}$ is the Fourrier coefficient that depends on $p_{\mathrm{T}}$. This function can be fit to the measured angular distributions of particles, for different $p_{\mathrm{T}}$ ranges, in order to obtain the $\nu_{2}$ values.

A non-vanishing $\nu_{2}$ indicates the presence of momentum anisotropy and, thus, the existence of a interacting medium. Its dependence on the momentum can be a sign of the different strengths of the interaction. This was shown for different particle species by STAR (figure 2.9 [3]) and PHENIX [4]. 


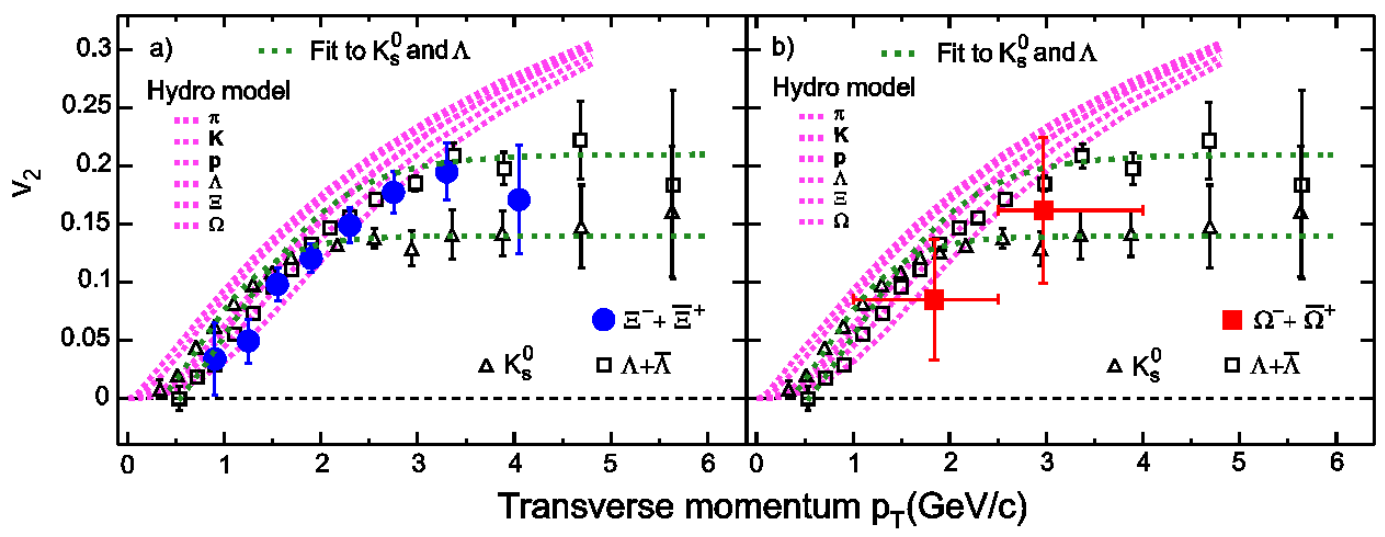

Figure 2.9: $\nu_{2}$, measured at STAR [3], as a function of their $p_{\mathrm{T}}$. In all cases, the anisotropy increases with the $p_{\mathrm{T}}$, what is expected from interacting with an spatially anisotropic medium.

\subsection{QGP in Small Systems}

Although several observables measured in $\mathrm{Pb}-\mathrm{Pb}$ with respect to $\mathrm{pp}$ collisions were reported, they might be unrelated to QGP or a colour-deconfined medium. One can use proton-nucleus collisions $(\mathrm{p}-\mathrm{Pb})$ as a benchmark [22] in order to probe sources of modifications, like the nuclei structure of the colliding ions, known generally as Cold Nuclear Matter (CNM) effect. It can affect initial state Parton Distribution Functions (assessed by modified PDFs or a Colour Glass Condensate (CGC) effective theory), initial and final state energy loss and the Cronin-like enhancement, due to multiple scattering (MPS).

In order to study these effects, a modified version of the $R_{A B}$ can be measured. Instead of calculating the ratio between $\mathrm{Au}-\mathrm{Au}(\mathrm{Pb}-\mathrm{Pb})$ and pp distributions ( $R_{A u A u}$ or $R_{P b P b}$ ), one can do the $\mathrm{p}-\mathrm{Pb}$ to $\mathrm{pp}$ one $\left(R_{p P b}\right)$, according to equation 2.11 , where $N_{\text {Coll }}^{p P b}$ is the number of binary collisions estimated by Glauber model calculations [23].

$$
R_{p P b}=\frac{1}{N_{\text {Coll }}^{p P b}} \frac{d N^{p P b} / d p_{\mathrm{T}}}{d N^{p p} / d p_{\mathrm{T}}}
$$

Since hard processes, which are related to high- $p_{\mathrm{T}}$ particles and jets, are expected to scale with the number of binary collisions, their $R_{p P b}$ must be around the unity. In figures 2.10 and 2.11, one can see that, for high- $p_{\mathrm{T}}$ charged particles and jets [24, 25], $R_{p P b} \approx 1$, an indication that the quenching observed in $\mathrm{Pb}-\mathrm{Pb}[3]$, related to the QGP, is not present in $\mathrm{p}-\mathrm{Pb}$.

Nevertheless, some unexpected effects were observed in pp and $\mathrm{p}-\mathrm{Pb}$ high multiplicity events. The CMS collaboration [26, 27] observed, in the $\Delta \eta \times \Delta \varphi$ two particle correlation function (figure 2.12), a near side ridge-like structure. They are similar to what was observed for heavy-ion collisions [28], that may indicate a collective behaviour of the particles also in small systems like pp and $\mathrm{p}-\mathrm{Pb}$. 


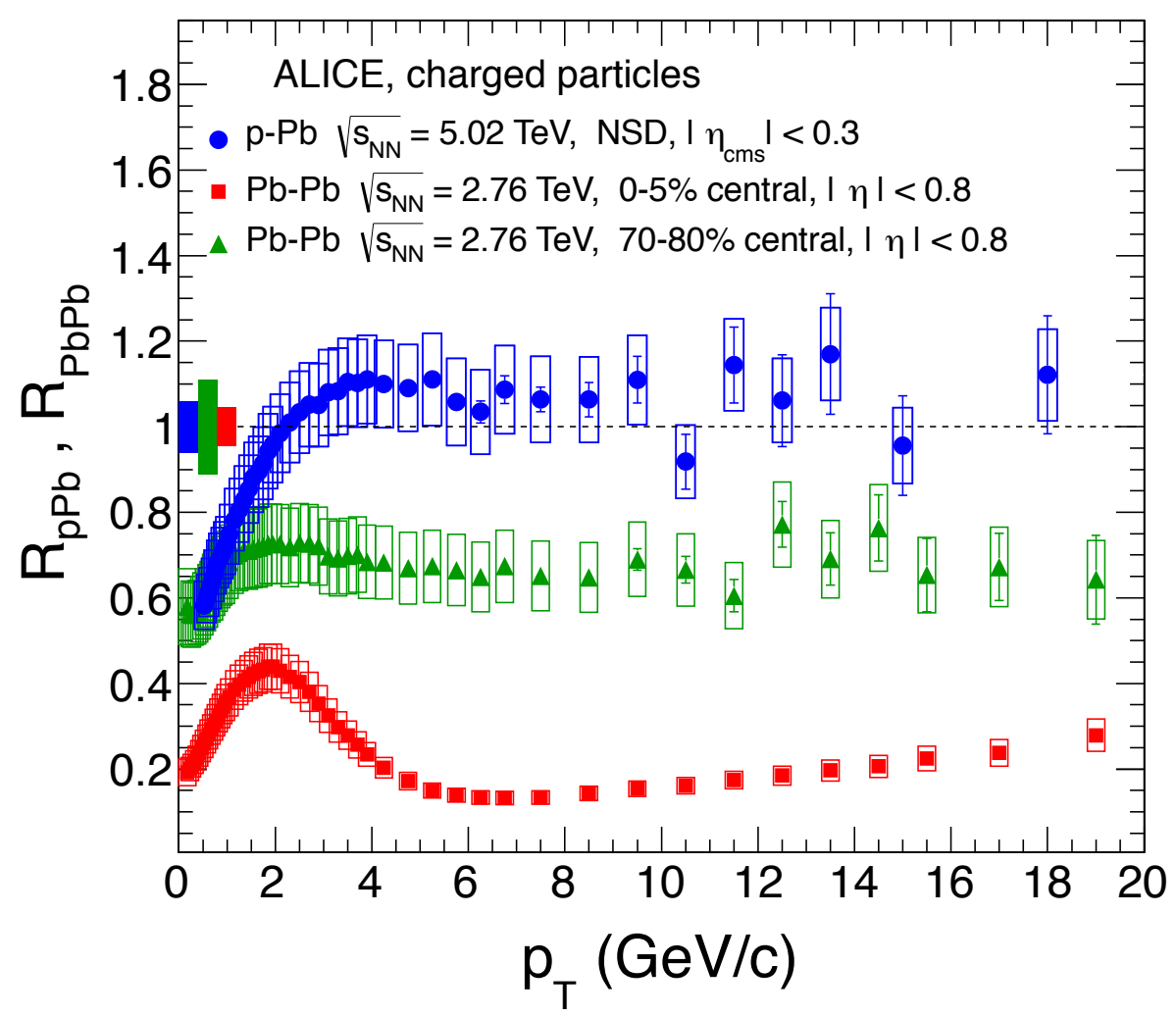

Figure 2.10: Charged particles $R_{p P b}$ (blue points) measured at ALICE [24]. They are compared to $R_{P b P b}$ results for two different multiplicity bins (red and green points), which indicates that, in $\mathrm{p}-\mathrm{Pb}$ collisions, the particles do not lose energy as in $\mathrm{Pb}-\mathrm{Pb}$ collisions.
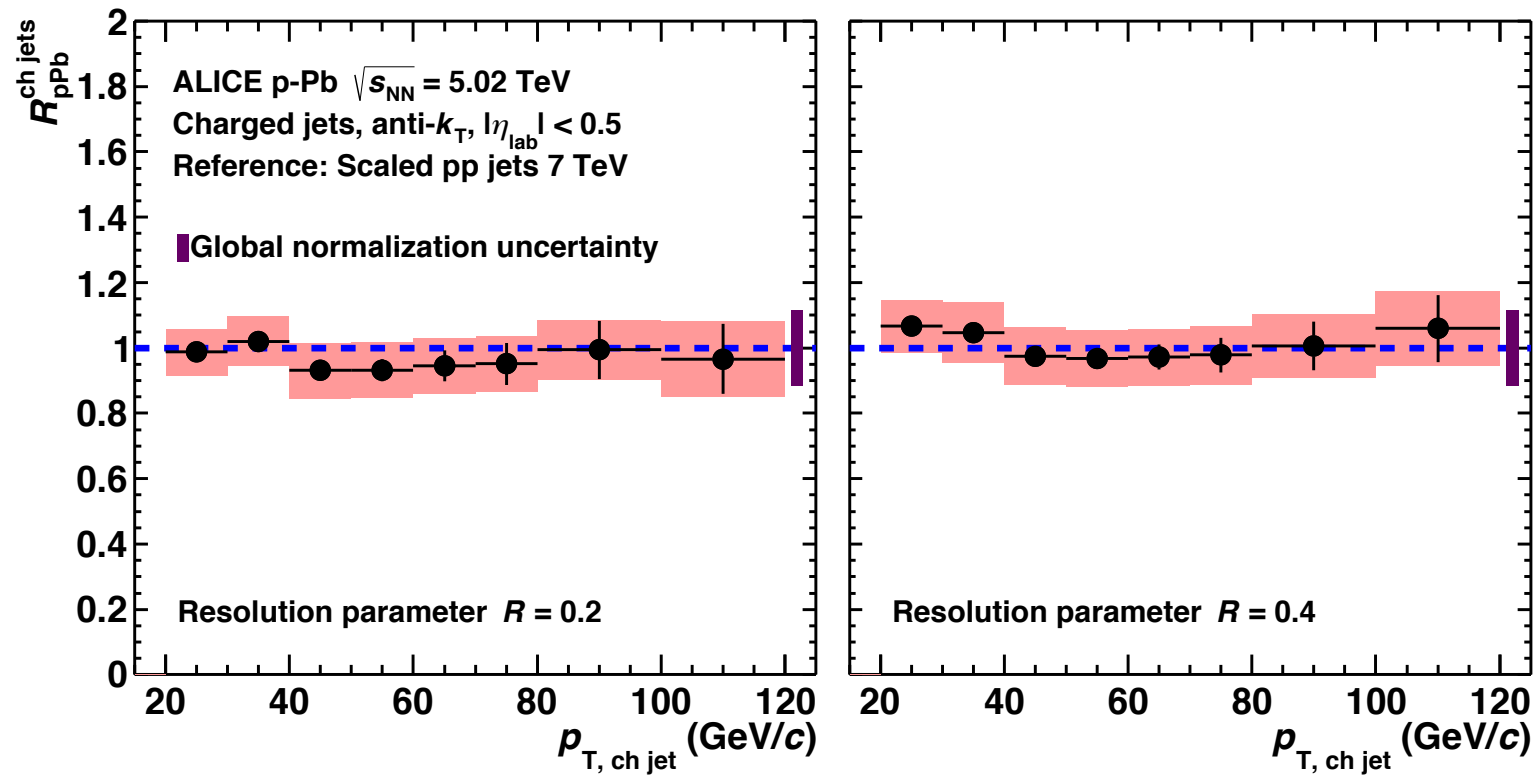

Figure 2.11: Jets $R_{p P b}$ measured at ALICE [25]. The values compatible with the unity indicate that jets lose energy similarly in $\mathrm{pp}$ and in $\mathrm{p}-\mathrm{Pb}$ collisions. 

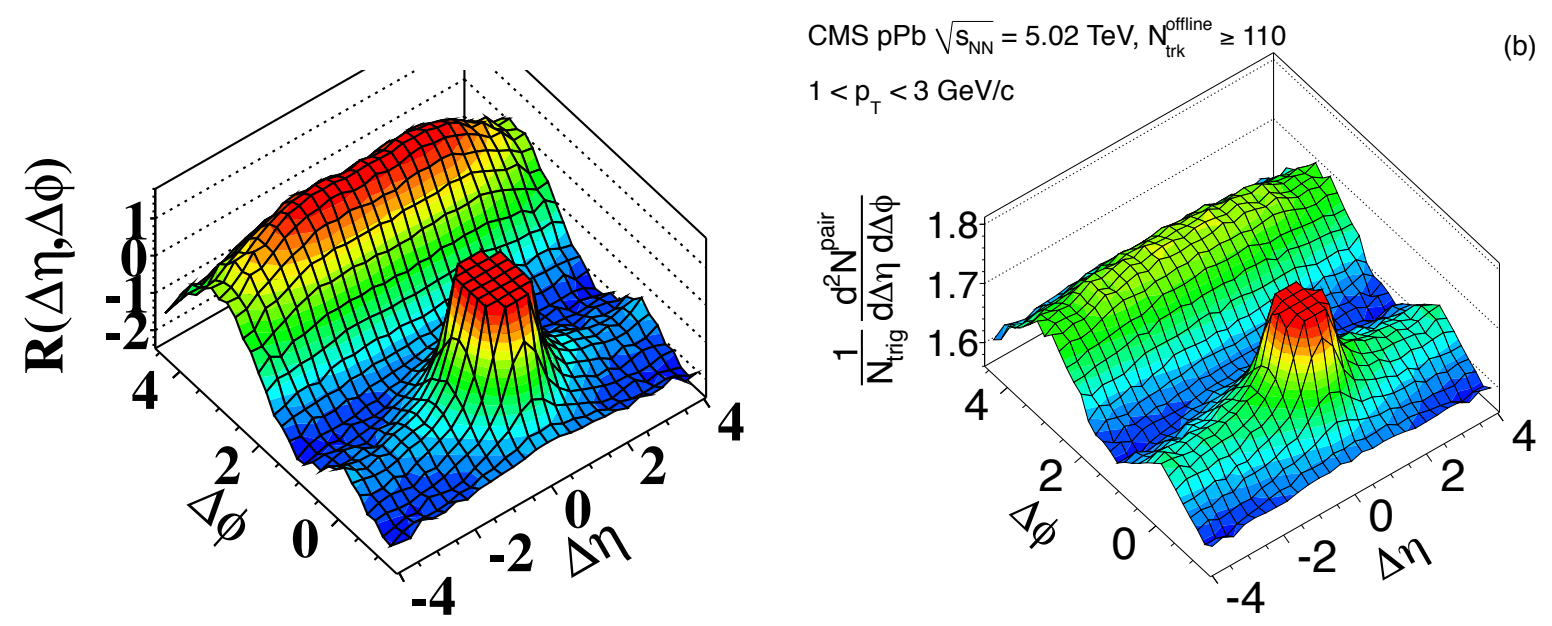

Figure 2.12: 2-D $(\Delta \eta \times \Delta \varphi)$ two particle correlation function, measured in pp collisions at $\sqrt{s}=7 \mathrm{TeV}$ (left-hand panel) [26], and in p-Pb events at $\sqrt{s}=5.02 \mathrm{TeV}$ (right-hand panel) [27], by CMS collaboration. In both cases, a ridge-like structure is visible in the near side region $(\Delta \varphi \approx 0)$, indicating a collective behaviour in those systems.

Besides being a reference to $\mathrm{Pb}-\mathrm{Pb}, \mathrm{p}-\mathrm{Pb}$ measurements may provide additional information on QGP properties and indicate other possible origins for the observed effects in all systems. Therefore, exploring more and new observables in $\mathrm{p}-\mathrm{Pb}$ may improve the understanding of the differences of the different collision systems. 


\section{Heavy Flavours and Jets}

In this chapter, two of the main probes to study the QGP are discussed: Heavy Quarks and Jets. Both probes are the result of hard scattering (high transfered momentum $Q^{2}$ ) and can be assessed in the experiment. The following sections will describe them, their possible observables and some results. The last section 3.5 will introduce the proposed observables that combine heavy quarks and jets, the subject of this thesis.

\subsection{Heavy Flavour Production}

The top (t), bottom (b) and charm (c) quarks are called heavy-flavour quarks because their masses are much larger than the up $(\mathrm{u})$, down $(\mathrm{d})$ and strange $(\mathrm{s})$ ones. This allows a different interpretation of their behaviour and the observables related to them.

Heavy-flavour quarks production must occur in the begining of the collision, when there is enough energy to be converted in their large masses. This makes them a good probe of the medium created by the collision: heavy flavour quarks interact with the medium during its complete existence since their lifetime $\left(10^{-11} \mathrm{~s}\right)$ is longer than the medium's one $\left(10^{-23} \mathrm{~s}\right)$ [29]. Their fragmentation is hard, i.e. they fragment in a heavyflavour meson that carries almost its total momentum, which facilitates the detection and selection of their products by the detectors. For the top quark, specifically, its lifetime is so short that it decays before fragmentating into mesons, which excludes it from the general heavy-flavour measurements.

Since their masses (and also the $Q^{2}$ ) are larger than the QCD scale $\Lambda_{Q C D}$, the process that generates the heavy-flavour quarks can be calculated using pQCD. The main and lowest-order Feynman diagrams, shown in figure 3.1, can be divided in three types of processes [30]: flavour creation (or pair creation), flavour excitation, and gluon splitting:

- Gluon Splitting: The $(Q \bar{Q})$ pair is created by a gluon, either in the initial or final state, and any of the quarks participate in the hard scattering, like in figure 3.1 ,f. The (transverse) momentum conservation favours the pair to be produced angularly close to each other.

- Flavour Excitation: When a non-valence (i.e. not in the mass shell) heavy-flavour quark in one beam interacts (hard scatters) with a parton from the other beam, it 


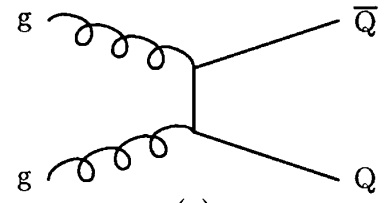

(a)

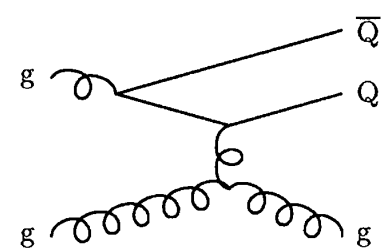

(d)

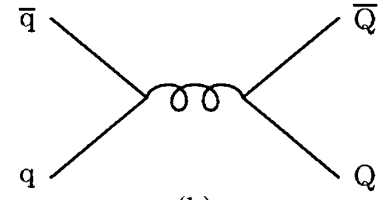

(b)

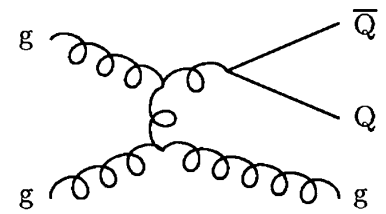

(e)

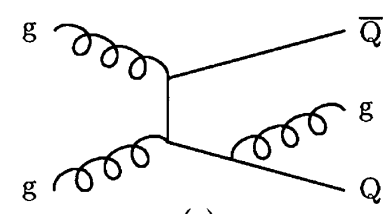

(c)

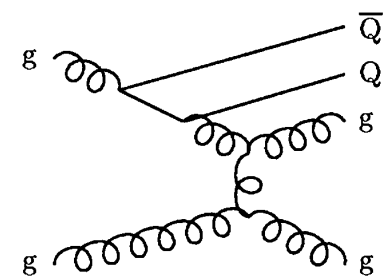

(f)

Figure 3.1: Heavy-flavour production Feynman diagrams [30]: (a) and (b) are flavour creation processes; (d) is a flavour excitation process; and (e) and (f) are gluon spliting processes.

can become a valence one. When it happens, the process is a flavour excitation. The non-valence quark can be created by a gluon splitting in the beam (figure $3.1 \mathrm{~d}$ ) before the hard scattering.

- Flavour Creation: The heavy-flavour quark-antiquark $(Q \bar{Q})$ pair is produced by leading order processes, like gluon fusion $(3.1 \mathrm{a}, \mathrm{c})$ and pair annihilation $(3.1 \mathrm{~b})$. The momentum conservation favours the pair to be produced in $\varphi$ opposition (back-toback);

The total production of heavy-flavour quarks is composed of all the three processes, in different fractions. According to the model in [30, at LHC energies, the flavour excitation, flavour creation and gluon splitting have similar contribution in the final cross section (figure 3.2). Since the angular distribution between the quark-anti-quark pair differs for each process, the back-to-back-like events and collinear-like events are mixed.

The fragmentation functions of charm and bottom quarks into mesons can be measured. Some examples of these values for charm, from HERA [31, can be seen in figure 3.3 , where it is shown that charm fragments in $D^{0}$ and $D^{+}$in more than $80 \%$ of the cases. The main heavy mesons $\left(D^{0}, D^{ \pm}, B^{0}, B^{ \pm}\right)$are the major products of the heavy-flavour quarks fragmentation and any of their decay channel can be used in a heavy-flavour measurement. In special, their semi-electronic decay, i.e. a decay with at least one electron, represents $\approx 10 \%$ of the cases, as shown in table 3.1, and is used in many analysis. 

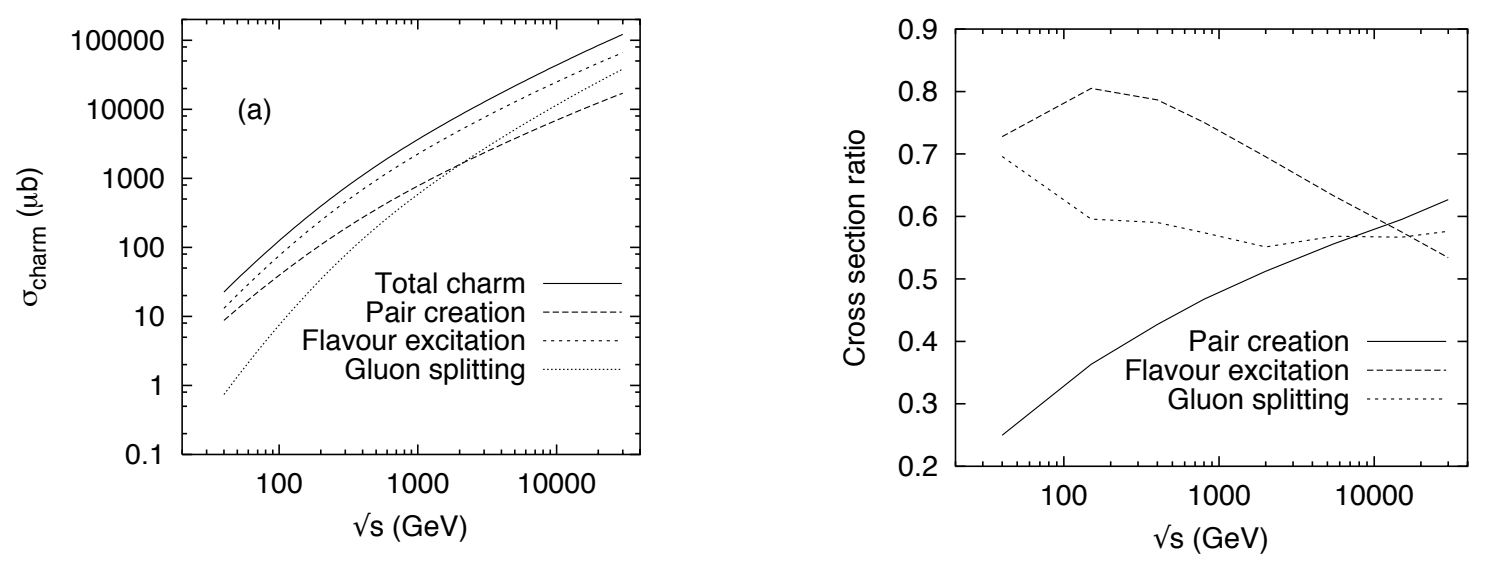

Figure 3.2: The charm production cross sections, for different processes, are compared (model from [30]): for LHC energies $(\approx 8 \mathrm{TeV})$, its production has similar contributions from flavour excitation, gluon splitting and flavour creation.

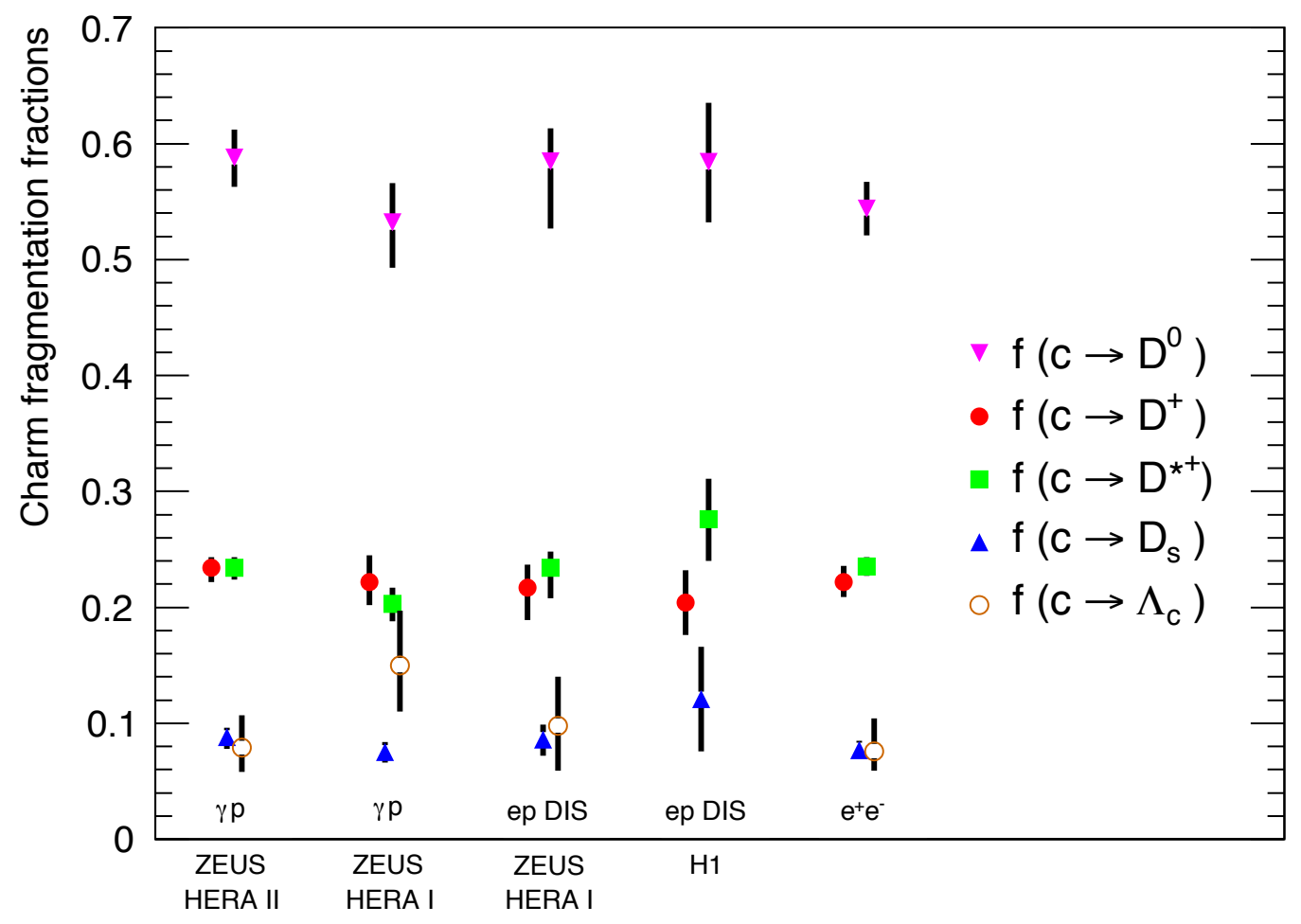

Figure 3.3: Charm fragmentation measured by HERA and other experiments [31]. The $D^{0}+D^{+}$ fraction represents more than $80 \%$ of the fragmentation cases. 
Table 3.1: Branching Ratio for Heavy Flavour Quarks [11]

\begin{tabular}{|c|ccc|}
\hline \hline Heavy Quark & Meson & Decay Channel & Branch Ratio \\
\hline \hline \multirow{4}{*}{$\mathrm{c}$} & \multirow{3}{*}{$\mathrm{D}^{+}$} & $\mathrm{e}^{+}+X$ & 0.1607 \\
& & $K^{-} \pi^{+} \pi^{-}$ & 0.257 \\
& & $e^{+}+X$ & 0.0904 \\
\cline { 2 - 4 } & \multirow{3}{*}{$D^{0}$} & $K^{-}+X$ & 0.0649 \\
& & $K^{-} \pi^{+}$ & 0.547 \\
& & $D^{0}+X$ & 0.0389 \\
\hline \multirow{4}{*}{$\mathrm{b}$} & \multirow{3}{*}{$B^{+}$} & $\bar{D}^{0}+X$ & 0.086 \\
& & $D^{+}+X$ & 0.79 \\
& & $D^{-}+X$ & 0.025 \\
& & $K^{ \pm}+X$ & 0.099 \\
& & $D^{0}+X$ & 0.78 \\
& $B^{0}$ & $\bar{D}^{0}+X$ & 0.081 \\
& & $D^{+}+X$ & $<0.474$ \\
& & $D^{-}+X$ & 0.369 \\
\hline \hline
\end{tabular}




\subsection{Heavy-flavour Measurements}

Several measurements were performed on correlations using either heavy-flavour observables or jets. In this section, one finds the $R_{A A}$ and $\nu_{2}$ results for heavy-flavour decay electron distributions, and jet spectra and jet-hadron observables results.

ALICE has measured heavy-flavour decay electrons observables as the azimuthal anisotropy 32 (figure 3.4). It shows a non-vanishing $\nu_{2}$, for a wide range of $p_{\mathrm{T}}$, which indicates that heavy-flavour decay electrons and their mother quarks present a collective behaviour in $\mathrm{Pb}-\mathrm{Pb}$ collisions.

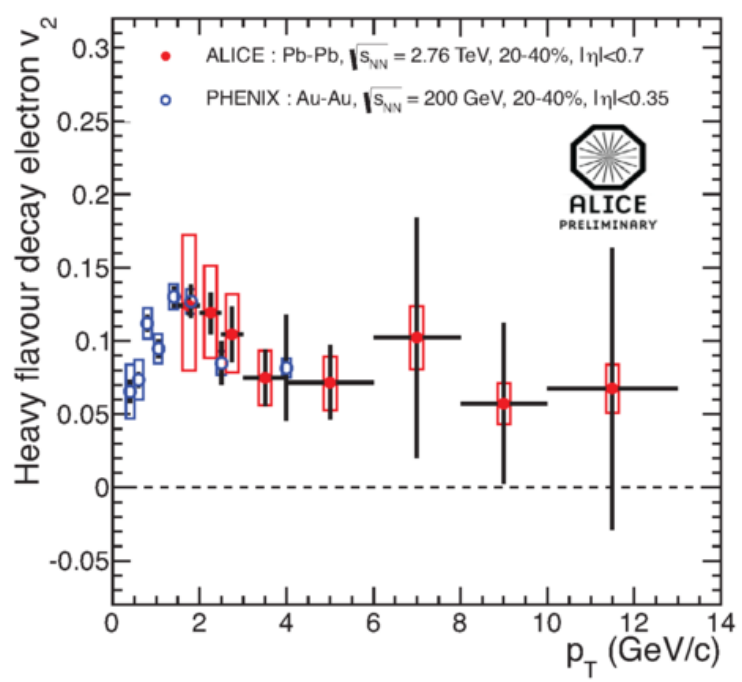

Figure 3.4: Heavy-flavour decay electrons $\nu_{2}$ measured at ALICE [32], for $\mathrm{Pb}-\mathrm{Pb}$ collisions at $\sqrt{s}=2.76 \mathrm{GeV}$. They are compared to PHENIX results (Au-Au at $\sqrt{s}=0.200 \mathrm{GeV}$, blue points). Both show non zero values, specially for the low momentum region.

The Nuclear Modification Factor $\left(R_{A B}\right)$ for the heavy-flavour decay electrons was measured for $\mathrm{p}-\mathrm{Pb}$ and $\mathrm{Pb}-\mathrm{Pb}$ data. ALICE [32] showed (figure 3.5, left panel) that there is a suppression for high $p_{\mathrm{T}}$ in $\mathrm{Pb}-\mathrm{Pb}$ data that indicates a energy loss process related to medium interaction for heavy-flavour quarks. Nevetheless, for p- $\mathrm{Pb}$ [33] this suppression is not observed (figure 3.5, right panel), indicating that there is no energy loss process in these collisions for heavy flavor quarks. 

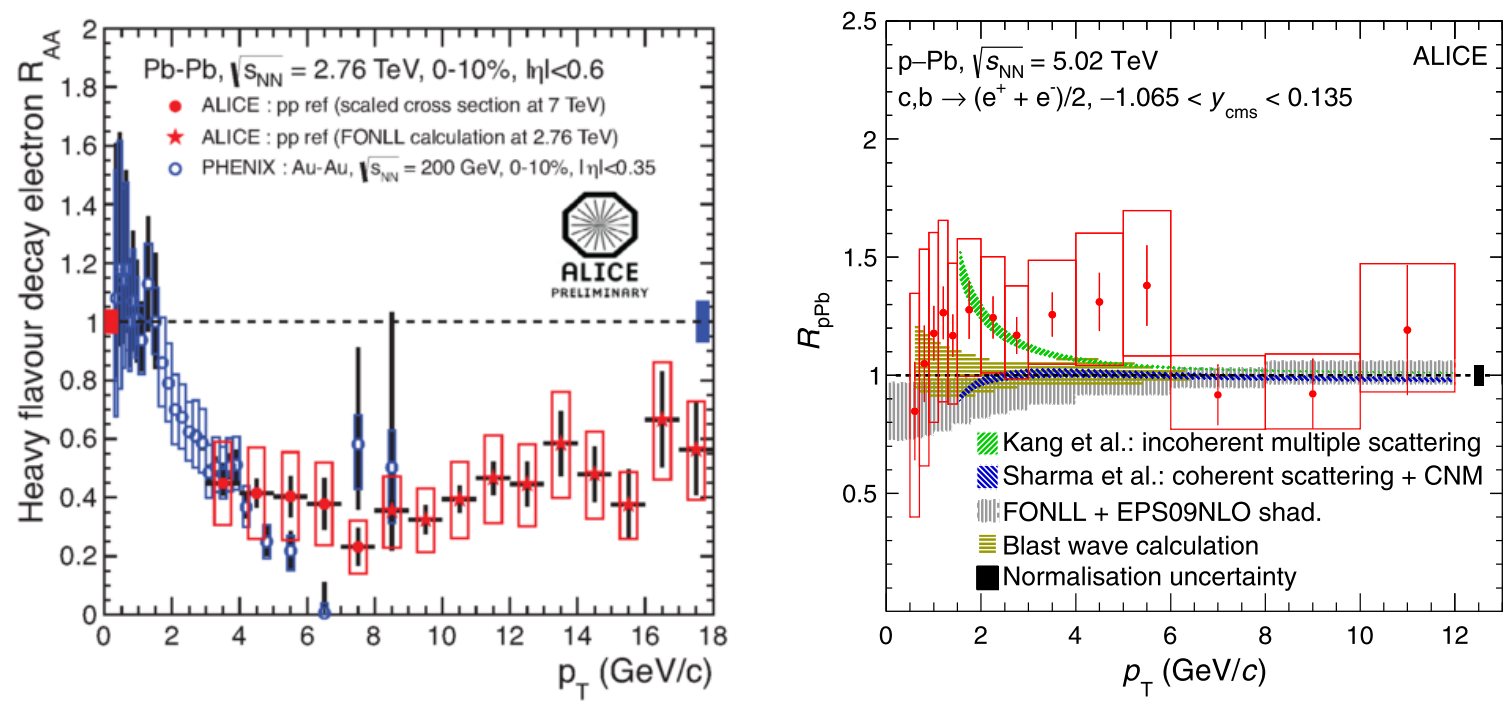

Figure 3.5: In the left panel, the heavy-flavour decay electrons $R_{A A}$, measured at ALICE [32] for $\mathrm{Pb}-\mathrm{Pb}$ collisions at $\sqrt{s}=2.76 \mathrm{GeV}$. They are compared to PHENIX results $(\sqrt{s}=0.200 \mathrm{GeV}$, blue points). For higher transverse momenta $(8-18 \mathrm{GeV} / c$ region), a FONLL pp reference is used instead the pp ALICE measurement $(3-8 \mathrm{GeV} / c$ region). In the right panel, the heavy-flavour decay electrons $R_{p P b}$ (red points), measured at ALICE [33], for p-Pb collisions at $\sqrt{s}=5.02$ $\mathrm{GeV}$.

\subsection{Jets}

In the hard scattering processes, the conservation of the four-momentum leads to a geometrical correlation between the produced particles. The first ones to be produced define some independent regions where all their fragmentation and decays, the so called parton shower, are collimated. These products, after hadronizing, can be grouped in what is called a jet. In this picture, the jet would represent the parton, i.e. the first significant product of the hard scattering, and could be used to study the collision. However, the definitions of partons and jets are ambiguous.

The usage of jets can be classified according to the origin of the parton that generates them [6].

In hadronic collisions, original partons, i.e. the partons inside the hadrons before they collide, scatter with one another $(2 \rightarrow 2)$ and the products are related to the inclusive jet spectrum. This kind of observable can be used to study the original parton distribution and the strengh of the interaction between them.

Another kind of parton that can generate a jet is a heavy quark. Massive particles, like top, bottom and charm quarks, produce a collimated spray after fragmenting and decaying, and these can be grouped into jets. An undiscovered massive particle, for instance, can be assessed by using jet momentum in invariant mass or kinematic missing energy calculations [6, 34].

The definition of jets can be done by choosing an algorithm for grouping (clusterizing) 
and another for combining the particles (recombination scheme). In the following sections, these two are discussed in hadronic collisions.

\section{Recombination Schemes}

The particles to be summed as a jet are defined by the clusterizing algorithm and, after that, a rule for merging two particles into a single object has to be defined. After the summation, the resulting object is called a pseudojet, that can be used again as an input to a new summation procedure or be classified as a final jet. In the following, two summation schemes are discussed: the Energy and the $p_{\mathrm{T}}$ schemes [6].

\section{Energy Scheme}

In this scheme, the four-momenta of particles are summed (equation 3.1) thus making the resulting four-momenta Lorentz invariant. In this case, the jets have mass $M=\sqrt{p^{\mu} p_{\mu}}$.

$$
\begin{gathered}
p_{r}^{\mu}=p_{1}^{\mu}+p_{2}^{\mu} \\
M_{r}^{2}=p_{r}^{\mu} p_{\mu, r}
\end{gathered}
$$

\section{$p_{\mathrm{T}}$ Scheme}

One can also dismiss the energy of the particles, i.e. ignore that they have masses, and use their geometrical information to compensate for it. In this case, $p_{\mathrm{T}}, \varphi$ and $y$ are used in the calculation of the resulting four-momentum (equation 3.2),

$$
\begin{aligned}
\overrightarrow{p_{\mathrm{T}, r}} & =\overrightarrow{p_{\mathrm{T}, 1}}+\overrightarrow{p_{\mathrm{T}, 2}} \\
\varphi_{r} & =\frac{\omega_{1} \varphi_{1}+\omega_{2} \varphi_{2}}{\omega_{1}+\omega_{2}} \\
y_{r} & =\frac{\omega_{1} y_{1}+\omega_{2} y_{2}}{\omega_{1}+\omega_{2}} \\
p_{r} & =\left\|\overrightarrow{p_{\mathrm{T}, r}}\right\|\left(\cosh \left(y_{r}\right), \cos \left(\varphi_{r}\right), \sin \left(\varphi_{r}\right), \sinh \left(y_{r}\right)\right)
\end{aligned}
$$

where a weight $\omega_{i}$ is used for summing the $i$-th particle variables to the $r$-th ones. The choice of $\omega_{i}$ is related to the clustering algorithm: it can be $\omega_{i}=\left\|\overrightarrow{p_{\mathrm{T}, i}}\right\|, \omega_{i}=\left\|\overrightarrow{p_{\mathrm{T}, i}^{2}}\right\|$, $\omega_{i}=E_{i}$, etc. In any case, the resulting jet mass is zero by construction.

\section{Clusterization Schemes}

A rule for grouping particles can deeply affect all jet measurements and observables. Thus, it is important to choose one according to the needs and the physical aim of a 
particular analysis. This was approached, in 1990, by some theorist and experimentalists that decided, in what is known as the Snowmass Accord, that any jet definition should include the following properties [35]:

1. Simple to implement in an experimental analysis;

2. Simple to implement in the theoretical calculation;

3. Defined at any order of perturbation theory;

4. Yields finite cross sections at any order of perturbation theory;

5. Yields a cross section that is relatively insensitive to hadronisation.

One can read, from these, two more practical properties: the infrared safety and collinear safety (figures 3.6 and 3.7). Being Infrared Safe means that the presence of low energy particles does not change the jet clusterization results, i.e. adding more low energy particles into an event does not change much the jets energies or positions. This is related to radiation that produces several particles with low momenta, but are still part of the jet. Being Collinear Safe means that the algorithm considers, in a similar way, one particle with a given energy and several particles with the same summed energy, when they are close in space ("collinear"). This relates to fragmentation, decays and hadronization, that changes the number of particles but their four-momentum is conserved in a given region. In addition, an infrared and collinear safe algorithm satisfies points 3,4 and 5 in the Snowmass Accord [6].
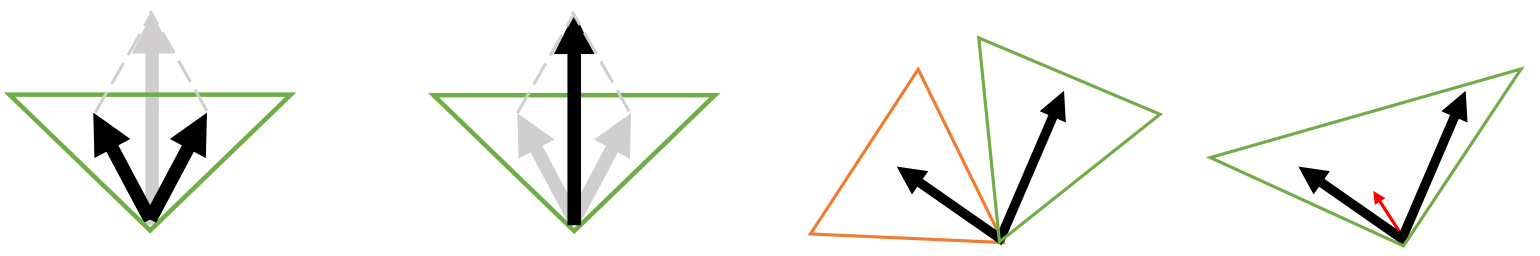

Figure 3.6: Collinear-safe algorithms in a pictoric scheme. Two collinear particles can be substituted by their sum, i.e. a particle whose momentum is the sum of the first two, without changing the reconstruction result.

Figure 3.7: Infrared-unsafe algorithms in a pictoric scheme. The inclusion of a low momentum particle (in red) changes the jet reconstruction result.

In the next section, some examples of algorithms will be explained, as well as some of their possible problems and solutions.

\section{Cone Algorithm}

The simplest idea for an algorithm consists in defining a region around a point and grouping all the particles inside it (equation 3.3). 


$$
\Delta R_{i}^{2}=\left(y_{i}-y_{\text {cone }}\right)^{2}+\left(\varphi_{i}-\varphi_{\text {cone }}\right)^{2}<R^{2}
$$

When one considers an event where all particles originate approximately from the same point, grouping particles around an angle looks like drawing a cone, thus the name cone algorithm. The first use of this kind of clustering was in electron-positron collisions [6], where one can clearly see few jets (mostly two) and there was almost no ambiguity. For hadron colliders, the event becomes more complex and dense in particles, revealing the problems in clusterizing using cone geometry only.

First of all, choosing the "radius" $R$ of the cone is arbitrary and can lead to partialy reconstructed jet or two jets reconstructed as one (overlaped jets), shown in fugure 3.8. The second problem is choosing the "seeds", the points where the clusterization begins: starting by a different point may lead to a completely different set of jets.
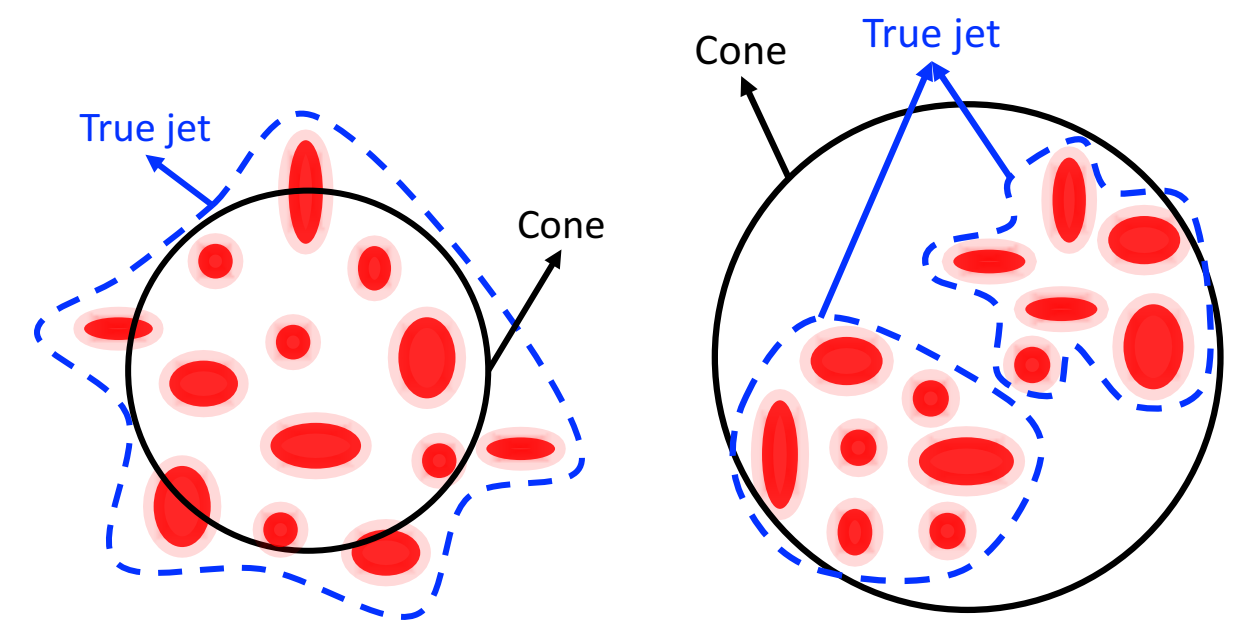

Figure 3.8: This pictoric jet reconstruction shows that a jet can be partially reconstructed (left-hand side) or two jets can be reconstructed as one (right-hand side).

In order to mitigate the effect of random seeds, one can iterate the clusterization. After grouping the particles and calculating the jet, the clusterization is re-done using the jet position as seed. This procedure can be done until the jet position is stable, when the next group of particles is clusterized in the same way until no particle is left ungrouped.

One approach to the overlapping problem is modifying it so the seed is the hardest particle that is still to be grouped and, after clusterizing, the particles in the new jet are excluded in the next iteration. From the remaining particles, one starts the algorithm with the hardest one and repeat the procedure until there are no particles to be clusterized. This progressive removal approach makes the cone algorithm colienar unsafe, since splitting a hard particle into two may lead to a different seed order and, thus, to a different set of jets.

Instead of removing the grouped particles, one can compare the jets that overlap and 
split the constituents between them or merge them. For example, if the fraction of $p_{\mathrm{T}}$ that is shared is greater than a certain value $f$, the jets must be merged; if it is not, the particles are associated to the closest jet. This split-merge approach is infrared unsafe because adding a soft particle in between two overlapping jets can lead to a single, merged jet instead of two, split jets.

There are several different implementations of a cone algorithm, using more diverse criteria for grouping, splitting and merging. Some may be collinear and infrared safe, like the SISCone algorithm [6], but the majority is not.

\section{$k_{T}$ and anti $-k_{T}$ Algorithms}

Another type of clustering algorithm is the sequential combination. In this case, the particles are sequentially combined in pairs and it is the algorithm that determines the next pair of particles (or pseudo-jets) to be combined into a new pseudo-jet. These combinations are iteratively done until no pseudo-jet satisfies the requirements to be merged, leaving a set of (final) jets. The rules followed in clusterization can be geometrical, kinematical, or both. The two most relevant algorithms of this kind are described in the following paragraphs: the (inclusive) $k_{T}$ and anti $-k_{T}$ algorithms [6].

Both algorithms use the distance in the phase space $y \times \varphi$ as a sequence criterion. This distance between the particles (or pseudo jets) $d_{i, j}$ (equation 3.4 ) is

$$
d_{i, j}=\min \left\{p_{\mathrm{T}, i}^{2 k}, p_{\mathrm{T}, i}^{2 k}\right\} \frac{\left[\left(y_{i}-y_{j}\right)^{2}+\left(\varphi_{i}-\varphi_{j}\right)^{2}\right]}{R}
$$

where $i$ and $j$ are two different particles (or pseudojets); and $k= \pm 1$ depending on the algorithm.

This distance is calculated for all particles in each iteration. If the smallest value $d_{r, s}$ is less than $d_{r, B}=p_{\mathrm{T}, r}^{2 k}$, the particles $r$ and $s$ are merged into a pseudojet. If it is not, the pseudojet $r$ is excluded from the clustering list and considered a final jet. The procedure is repeated for the new pseudojets and particles until no pseudojet is left. The parameter $k$ is 1 for $k T$ algorithm and -1 for anti $-k T$.

The only difference between (inclusive) $k_{T}$ and ant $i-k_{T}$ algorithms is in the calculation of $d_{i, j}: k_{T}$ favours grouping around the low $p_{\mathrm{T}}$ pseudojets because the distance is proportional to the transverse momentum. On the other hand, anti $-k_{T}$ favours grouping around high $p_{\mathrm{T}}$ since the distance is inversely proportional to the transverse momentum.

These two algorithms are infrared and collinear safe and have simple implementation, which grants them a wide use in the jet field. This analysis uses the $k T$ and anti $-k T$ algorithms and the $p_{\mathrm{T}}$ recombination scheme with weight $\omega=p_{\mathrm{T}}$, as it will be explained later. 


\subsection{Unfolding}

The measurement of any observable has always effects from the detectors and other phenomena. For a functional observable, i.e. an observable that is composed of a continuous function, one can describe (equation 3.5) the measured observable $m$ by a convolution of a true observable $t$ and a function of effects $A$, the response matrix. [6, 36, 37].

$$
m(x)=\int A(x, \mu) t(x) d \mu
$$

In order to obtain the "true" measured observable, one has to invert equation 3.5 , the so called unfolding procedure. This is important to compare the measured result with measurements from other experiments, since the unfolded distribution does not include detector effects. Presenting unfolded results is also better when comparing to theory because no extra information is needed but the result itself.

For the jet spectra case, the measured observable $m$ is a N-bins histogram, i.e. a vector with $\mathrm{N}$ entries. Therefore, one can rewrite equation 3.5 using a discrete formulation as in equation 3.6 .

$$
m_{i}=A(\mu)_{i, j} t_{j}
$$

The inversion of the matrix equation is simpler (equation 3.7), being the most difficult part the matrix $A$ inversion.

$$
t_{j}=A(\mu)_{i, j}^{-1} m_{i}
$$

Specially for measurements with large uncertainties, the process of inverting matrices can lead to great variation and increase background and noise effects. For minimizing these fluctuations in the unfolded spectra, a regularization procedure is used [36, 37], although a bias must be introduced by choosing an estimate of the true spectrum.

In this thesis, two unfolding methods are used: Iterative Bayesian and Single Value Decomposition (SVD) [6].

\subsection{Track-Jet Analysis}

Reconstructed jets, due to their algorithm nature, can be a product of a hard process (a real, signal jet) but can be also related to non-hard process, i.e. simply a bunch of particles, created by low momentum transfer interaction, that were grouped together. A mix of jets from different origins can lead to wrong conclusions and, thus, some selection must be performed.

One can, for example, require a high $p_{\mathrm{T}}$ track inside the jets to consider them for the 
measurement or apply a cut in the jet $p_{\mathrm{T}}$. One drawback of such procedure is favouring harder jets that can bias the measurement towards less medium interaction and excluding low energy jets. In order to avoid undesirable bias on jets, one can require a high $p_{\mathrm{T}}$ track in opposition to the jet, i.e. in the away side.

\section{Hadron-Jet Coincidence Analysis}

High $p_{\mathrm{T}}$ hadrons are products of hard scattering events. The conservation of the (transverse) momentum favours the occurence and reconstruction of jets in a back-toback situation. For this reason, a cut in the hadron $p_{\mathrm{T}}$ induces a bias in the jet $p_{\mathrm{T}}$ that can be explored to select a specific set of jets.

Therefore, hadrons and jets in geometric opposition can be paired, i.e. the the condition $\Delta \varphi_{h, j e t}-\pi \mid \approx 0$ can be required, in order to obtain a semi-inclusive jet spectrum [38, 39], that is called hadron-jet coincidence measurement.

In the hadron-jet coincidence measurement, the bias would not affect the jet energy or momentum directly. This kind of selection can even favor the jets that go through the medium, since high- $p_{\mathrm{T}}$ hadrons are very likely to be produced in the medium boundaries, so it has a small energy loss.

Although this observable should have a lower background of combinatorial jets, they are still present and must be subtracted. An approach to this is to use two different $p_{\mathrm{T}}$ intervals $I_{1}, I_{2}$ for the away side hadron [39] thus measuring two different observables $\left(\frac{d^{2} N^{h \in I_{1}}}{d \eta d p_{\mathrm{T}}}\right.$ and $\left.\frac{d^{2} N^{h \in I_{2}}}{d \eta d p_{\mathrm{T}}}\right)$ with the same background $\frac{d^{2} N^{b k g}}{d \eta d p_{\mathrm{T}}}$. The difference between these two quantities (equation 3.8) represents a signal-only observable. This observable is shown in figure 3.9 [39], where the intervals are $I_{1}=\left[8,9\left[\mathrm{GeV}\right.\right.$ and $I_{2}=[20,50[\mathrm{GeV}$.

$$
\begin{aligned}
\frac{d^{2} N^{h \in I_{1}}}{d \eta d p_{\mathrm{T}}} & =\frac{d N^{\text {signal } 1}}{d p_{\mathrm{T}}}+\frac{d^{2} N^{b k g}}{d \eta d p_{\mathrm{T}}} \\
\frac{d^{2} N^{h \in I_{2}}}{d \eta d p_{\mathrm{T}}} & =\frac{d N^{\text {signal 2 }}}{d p_{\mathrm{T}}}+\frac{d^{2} N^{b k g}}{d \eta d p_{\mathrm{T}}} \\
\Delta_{\text {recoil }} & =\frac{d^{2} N^{h \in I_{2}}}{d \eta d p_{\mathrm{T}}}-\frac{d^{2} N^{h \in I_{2}}}{d \eta d p_{\mathrm{T}}}
\end{aligned}
$$

Another approach to subtracting the combinatorial jets is the mixing event technique [39, 40]. One creates a completely fake event by combining tracks from different real events, and analyses it with the same procedures in order to find a combinatorial only spectrum. This mixed event spectrum can be subtracted from any $\frac{d^{2} N^{h \in I}}{d \eta d p_{\mathrm{T}}}$ resulting in a signal-only observable (figure 3.10 [40]). 

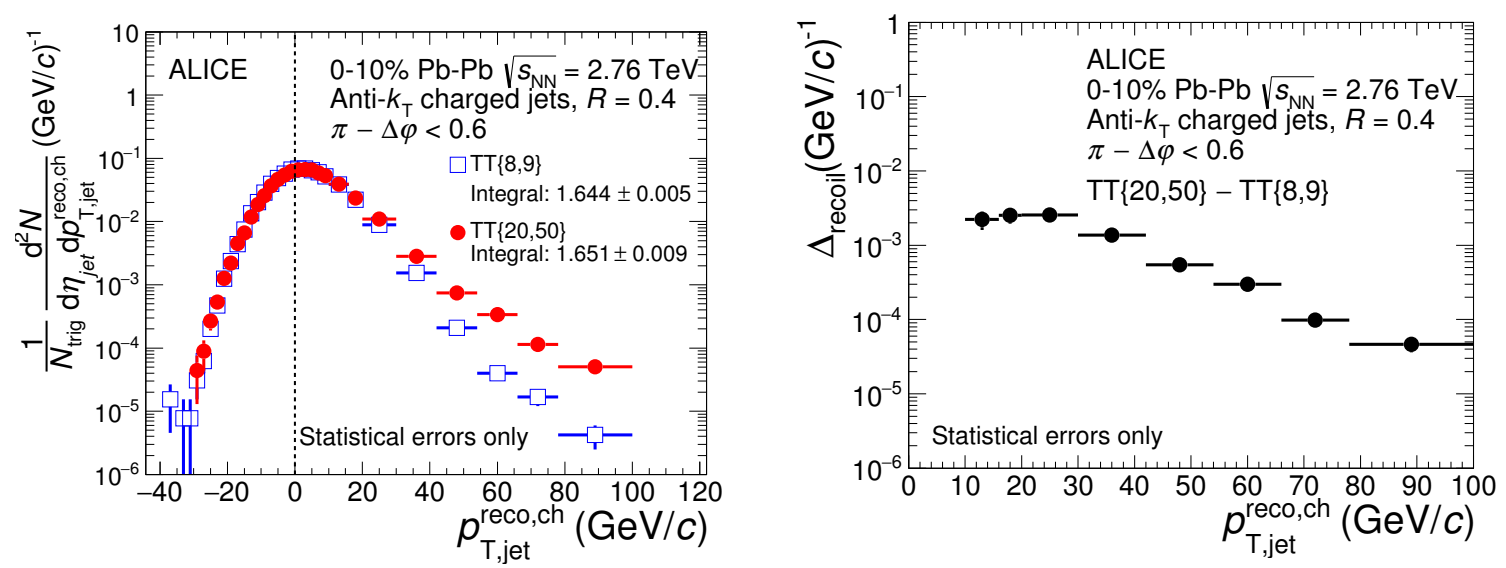

Figure 3.9: In the left panel: jet spectra for different $p_{\mathrm{T}}^{\text {trigger }}$ intervals [39]. In the right panel, the difference between the two spectra $\Delta_{\text {Recoil }}$.

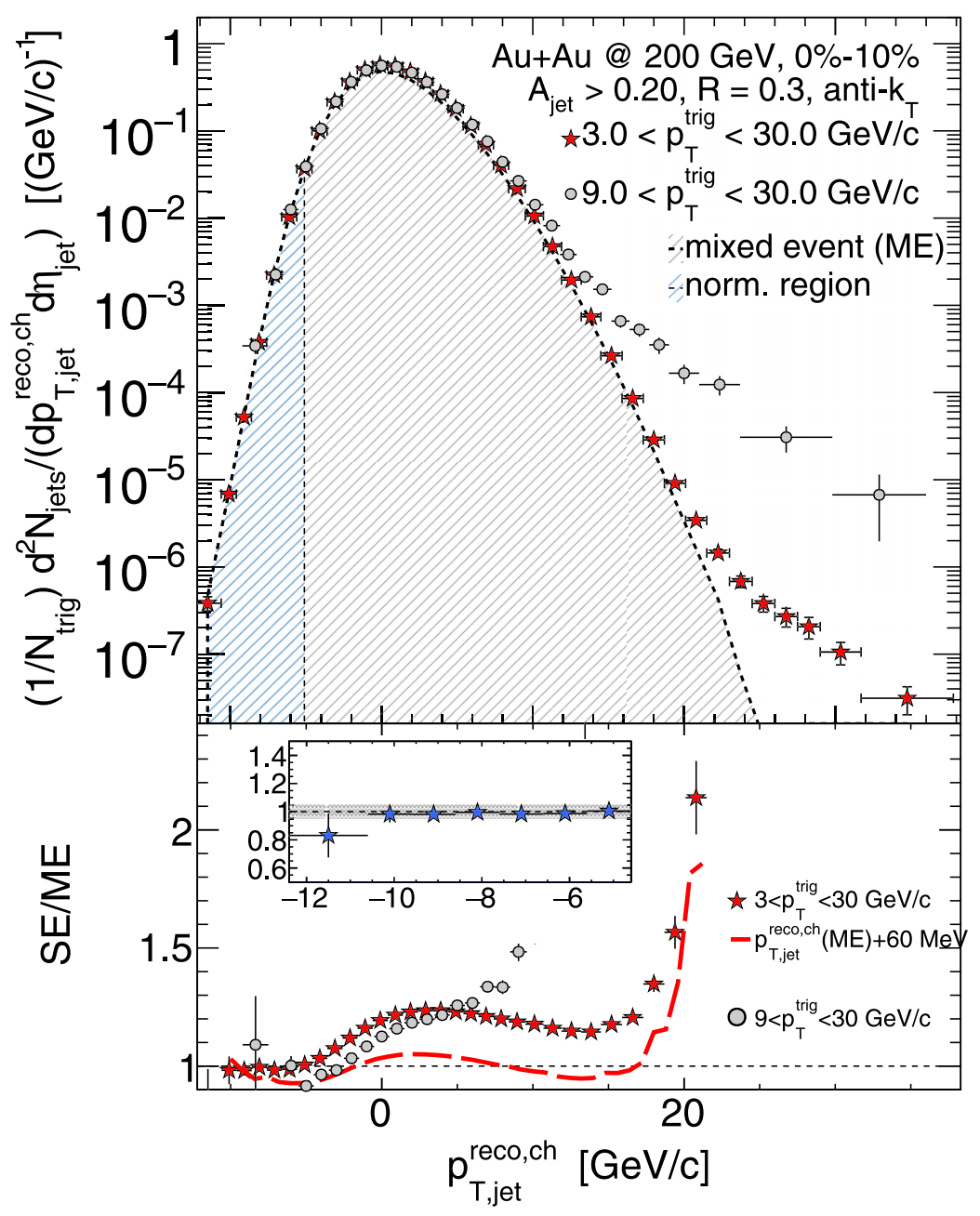

Figure 3.10: In the top panel, semi-inclusive jet spectrum for two trigger intervals (gray and red points) and the event mixing correction (gray shaded area) measured by STAR [40]. The bottom panel show the ratio between the spectra and the mixed event correction. The event mixing contribution can be subtracted in order to obtain a signal-only observable. 
From the hadron-jet pairs, one can obtain an angular distance $\Delta \varphi=\left\|\varphi_{\text {hadron }}-\varphi_{\text {Jet }}\right\|$ distribution also for different $p_{\mathrm{T}}$ intervals. The result is in figure 3.11 , where a signal only, angular distribution is shown. This kind of analysis may be used to measure semi-inclusive jet suppression for $\Delta \varphi$ intervals.

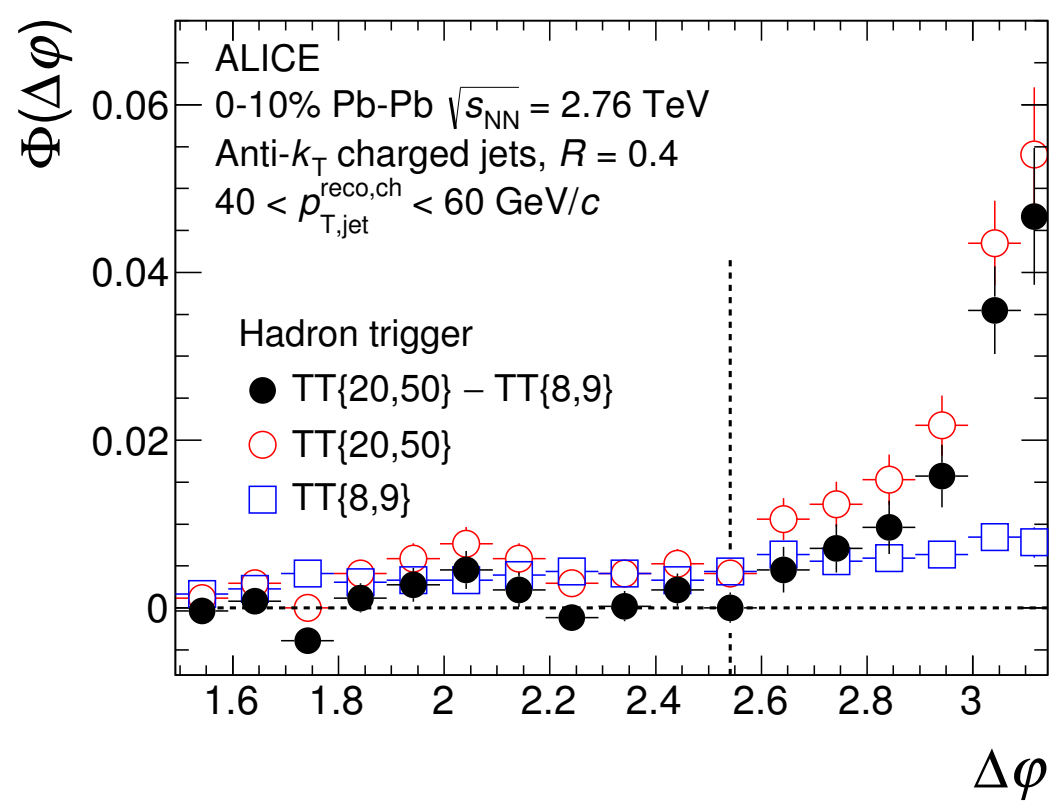

Figure 3.11: Hadron-Jet $\Delta \varphi$ [39] for different $p_{\mathrm{T}}^{\text {trigger }}$ ranges can be used to study the hadron-jet distribution shape or the azimuthal anistropy.

A similar jet-hadron correlation measurement was performed by STAR [41], comparing pp and $\mathrm{Au}-\mathrm{Au}$ results. Figure 3.12 shows their angular distribution, for two different associated hadron transverse momenta $p_{\mathrm{T}}^{a s s o c}$ intervals, to which two Gaussian curves (near and away side peaks) and a modulated background were fitted.

The width of the Gaussians and their area (Yield) can quantify the medium induced modifications. Figure 3.13 shows: in the top panel, the distribution of the away side widths as a function of hadron $p_{\mathrm{T}}^{a s s o c}$; and in the bottom panel, the difference between $\mathrm{pp}$ and $\mathrm{Pb}-\mathrm{Pb}$ away side yields $D_{A A}$ as a function of hadron $p_{\mathrm{T}}^{\text {assoc }}$. The widths values indicate that for low $p_{\mathrm{T}}^{a s s o c}$, there is a broadening for the distribution, that is not observed for higher $p_{\mathrm{T}}^{a s s o c}$. The $D_{A A}$ values indicate that jets in $\mathrm{Pb}-\mathrm{Pb}$ are significantly softer than in $\mathrm{pp}$ (enhancement in the low $p_{\mathrm{T}}^{a s s o c}$ region).

In this work, a similar approach to select jets is adopted. As discussed in the next section, the proposition is to substitute the high- $p_{\mathrm{T}}$ hadron by a heavy-flavour decay electron. 


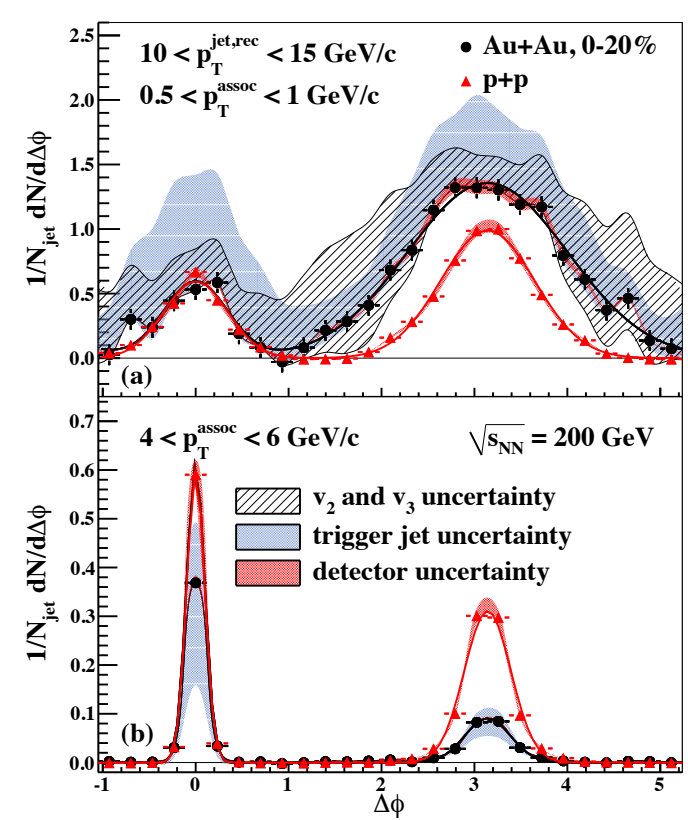

Figure 3.12: Jet-hadron $\Delta \varphi$, measured at STAR 41. Two Gaussians and a modulated background are fit to the data. In the top panel, the associated particle have $p_{\mathrm{T}}^{\text {assoc }} \in[0.5,1.0] \mathrm{GeV} / c$. In the bottom panel, $p_{\mathrm{T}}^{\text {assoc }} \in[4,6] \mathrm{GeV} / c$

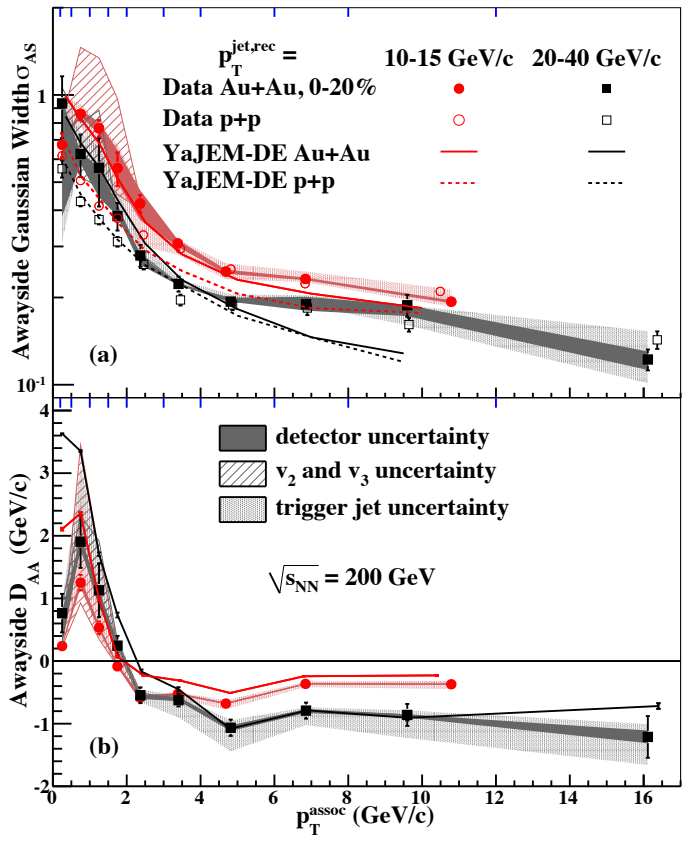

Figure 3.13: Jet-hadron medium modification observables, measured at STAR [41. In the top panel, the distribution of the away side widths as a function of hadron $p_{\mathrm{T}}^{a s s o c}$ is shown. In the bottom panel, the difference between pp and $\mathrm{Pb}-\mathrm{Pb}$ away side yields $D_{A A}$ as a function of hadron $p_{\mathrm{T}}^{a s s o c}$.

\section{Electron-Jet Correlations}

Similarly to high- $p_{\mathrm{T}}$ hadrons, heavy-flavour quarks are originated in hard scattering processes and can be used to obtain a semi-inclusive jet spectrum and an angular correlation distribution. Their presence can be infered via heavy-flavour decay electrons, which can be paired to jets. These pairs have an additional bias towards selecting heavy-flavour jets, in the near and away side regions. The aim of this analysis is to study the electron-jet observables, and the proposed methods are described in this section.

As shown in table 3.1 , the study of heavy flavor quark production through the electron channel is specially relevant, since $\approx 10 \%$ of charm quarks have an electron as a decay product. In addition, the high- $p_{\mathrm{T}}\left(p_{\mathrm{T}}>1 \mathrm{GeV} / c\right)$ electron production is completely dominated by heavy-flavour decays, with a small fraction of light-flavour origin background and electrons from conversions in the detectors materials. Besides that, ALICE has a good electron identification purity [42] which makes it a good choice as the trigger for a hard scattering process and substitute for the high- $p_{\mathrm{T}}$ hadron.

There are two main sources of background in the electron production measurement: electrons misidentified as hadrons (hadronic contamination); and the low invariant mass electron-positron pairs (conversion and Dalitz decay contamination). 
- Hadronic Contamination: The particle identification with energy loss measurements in the TPC has ambiguities that must be considered. In order to take this contamination into account, the EMCal energy information is used as a second constraint;

- Conversions and Dalitz decay contamination: The interaction of photons mainly coming from $\pi^{0}$ decays with any material of the detector can result in a electronpositron pair creation. These electron-positron pairs have a small invariant mass $\left(m_{e^{+} e^{-}}^{\gamma}=0\right.$ and $\left.m_{e^{+} e^{-}}^{\pi^{0}}<m_{\pi} \approx 0.135 \mathrm{GeV} / c\right)$, and can be statistically removed using a cut in the mass value. A similar procedure can be applied for electrons coming from light-flavour mesons $\left(\pi^{0, \pm}\right)$ Dalitz decay;

The heavy-flavour decay electrons are paired to the reconstructed jets in the event so their angular distance distribution $\frac{d^{2} N^{H F e-j e t}}{d \eta d \Delta \varphi}$ can be measured. This distribution is obtained for different electron $p_{\mathrm{T}}^{e}$ intervals, namely $[3,4[$, $[4,6[$, and $[6,18[\mathrm{GeV} / c$, and inclusive charged jet $p_{\mathrm{T}, \text { chjet }}\left(p_{\mathrm{T}, \text { chjet }} \in\left[10,80[\mathrm{GeV} / c)\right.\right.$. A jet $p_{\mathrm{T} \text {,chjet }}$ dependence can be assessed with the semi-inclusive jet spectrum, that is a selection of jets for a $\Delta \varphi$ range (near or away side) and inclusive electron $p_{\mathrm{T}}\left(p_{\mathrm{T}}^{e} \in[3,9[\right.$ or $[3,18[\mathrm{GeV} / c)$.

The correlation between the heavy-flavour meson and its decay electron momenta are minimum, due to the multiple $(>2)$ particle decay. For any given range of the electron $p_{\mathrm{T}}^{e}$, the meson $p_{\mathrm{T}}^{\text {meson }}$ spams throughout all possibilities $\left(p_{\mathrm{T}}^{\text {meson }} \geq p_{\mathrm{T}}^{e}\right)$. This is not observed when using hadrons, which have a clear $p_{\mathrm{T}}$ correlation with mesons. Therefore, the use of different electron $p_{\mathrm{T}}^{e}$ ranges for selecting different signal jets (like the $I_{1}$ and $I_{2}$ intervals used in the hadron-jet coincidence analysis) is not feasible and other background subtraction method is needed.

The proposed observable $\frac{d^{2} N^{\text {jet, side }}}{d \eta d p_{\text {T,chjet }}}$ selects the electron-jet (HFe-jet) pairs that have, at least, a $\Delta \varphi=\pi / 2$ away side signal spectrum (side = away), or $\Delta \varphi<\pi / 10$ near side signal spectrum (side = near). An approach to estimate the non-signal contribution is to calculate the spectrum of HFe-jet pairs that are "perpendicular" to each other or in the middle between the near and the away sides. So, instead of $\Delta \varphi>\pi / 2$, the background spectrum $\frac{d^{2} N^{\text {jet }, \text { middle }}}{d \eta d p_{\mathrm{T}}}$ is composed of selected pairs with $\pi / 10<\Delta \varphi<\pi / 2$. Their subtraction $\Delta_{\text {side }}$ is in equation 3.9 , where the angular normalization $A_{\varangle}$ accounts for the different selection regions in $\varphi$.

$$
\Delta_{\text {side }}=\frac{1}{N_{H F e}^{\text {side }}} \frac{d^{2} N^{\text {jet,side }}}{d \eta d p_{\mathrm{T}, \mathrm{chjet}}}-\frac{A_{\varangle}}{N_{H F e}^{\text {middle }}} \frac{d^{2} N^{\text {jet,middle }}}{d \eta d p_{\mathrm{T}, \mathrm{chjet}}}
$$

In chapter 6, the details of the measurement of the two observables, namely the semiinclusive HFe-jet spectrum and the HFe-jet angular difference distribution, will be discussed for pp data. In chapter 7, the details for $\mathrm{p}-\mathrm{Pb}$ data are discussed. Some discussion on comparing pp and $\mathrm{p}-\mathrm{Pb}$ results is in chapter 8 . 


\section{A Large Ion Collider Experiment}

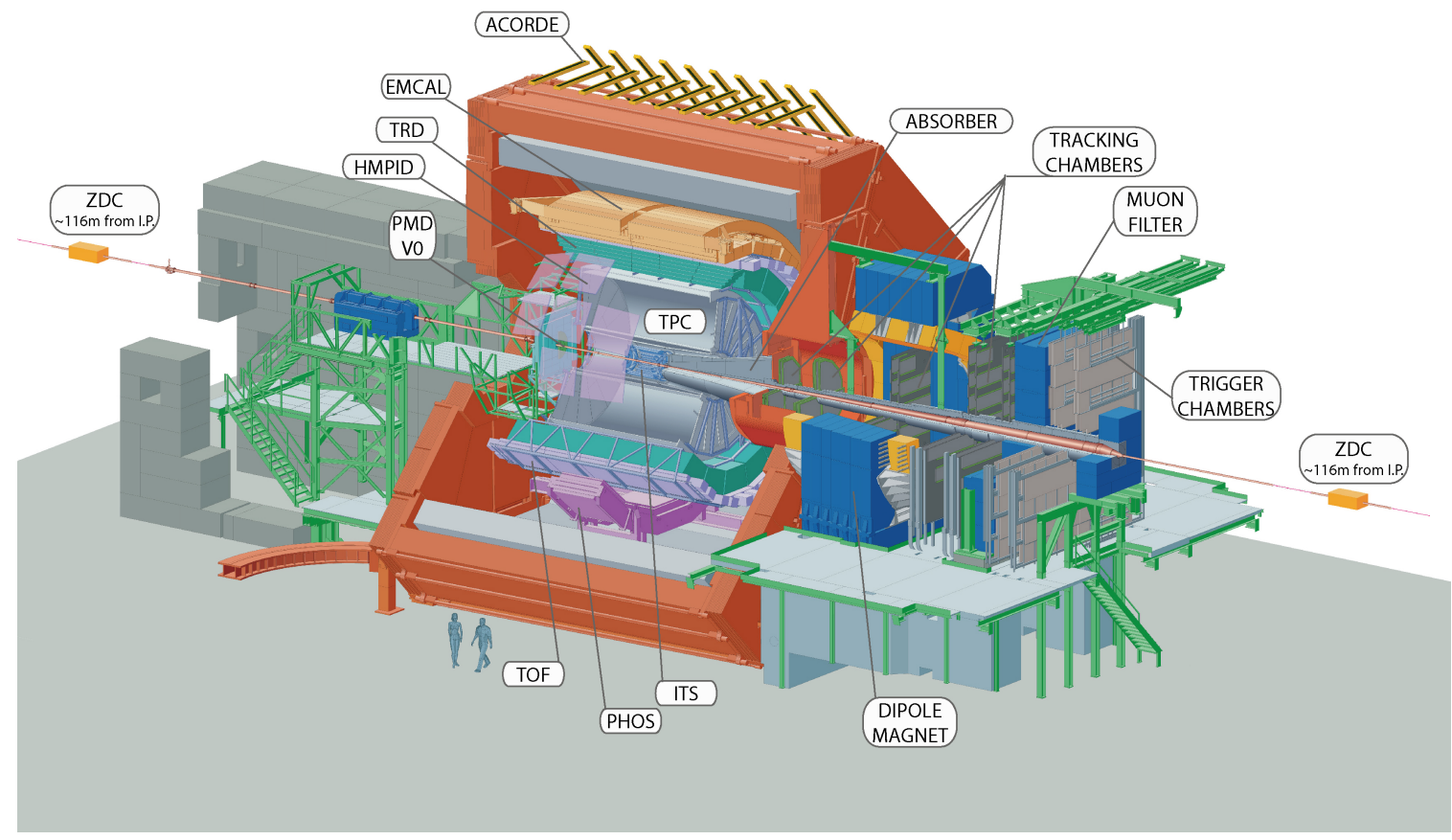

Figure 4.1: Scheme of the ALICE detectors [43]. The main systems have their names indicated in the figure. Humans were drawn in the bottom, left part as a reference to the size scale.

A Large Ion Collider Experiment (ALICE) is one of the experimental collaborations at the Large Hadron Collider (LHC), in the European Organization for the Nuclear Research (Organisation Européen pour la Recherche Nucléaire, CERN). It was designed to measure lead nuclei collisions in an optimal way and its subsystems, or detectors, are projected to detect the signals, process and record a huge amount of data collected in each event. Figure 4.1 shows a scheme of ALICE detectors.

All the detectors in the central barrel region, i.e. the region for $|\eta| \lesssim 1$, namely the Inner Tracking System (ITS), the Time-Projection Chamber (TPC), the Electromagnetic Calorimeter (EMCal) and the Time of Flight (ToF), are immerse in a magnetic field [44] of $0.5 \mathrm{~T}$ in the longitudinal direction. It has an important role in calculating the charged particles momenta. 
A standard track is reconstructed using only TPC clusters, since it is the most common and probable situation. This includes the tracks used in jet reconstruction, although it does not exclude other possibilities. The main particle identification technique uses the energy loss pattern in the TPC. For the electron case, one can use the energy deposited in the EMCal or the ToF signal in order to improve the selection, thus requiring clusters in these detectors.

In the following section, some details about the LHC operation will be shown. In the next sections, one finds some specific characteristics of the ITS, TPC, V0 and EMcal detectors since they are the most relevant ones for this analysis. For more details on all the detectors, see [44], [45] and [46].

\subsection{Large Hadron Collider}

The Large Hadron Collider (LHC) is a 27-kilometer-long ring that accelarates protons and lead nuclei $(\mathrm{Pb})$. This structure is located in the France-Switzerland border (swiss canton of Geneva and the french department of Ain), partially inside the European Organization for Nuclear Ressearch's (CERN's) site.

Proton beams are accelerated in stages [47, 48]. The first one corresponds to the linear accelerator (Linac) where the beams reach the energy of $50 \mathrm{MeV}$. This beam is, then, injected in the Proton Syncrotron Booster, that increases its energy to $1.4 \mathrm{GeV}$, and, later, in the Proton Syncroton itself, where the beam energy reaches $25 \mathrm{GeV}$. The next stage is in the Super Proton Syncrotron, that pushes the beam energy to $450 \mathrm{GeV}$.

The final acceleration in done inside the LHC, where the beam energy goes up to 6.5 GeV. Two beams are injected in the LHC, one in each direction, clockwise and anticlockwise. A similar procedure for lead nuclei is performed, so the collisions between protons, proton and lead, and between lead nuclei can be recorded. All the complex is shown in figure 4.2 .

In 2010, the LHC became operational and the experiments started recording protonproton (pp) collisions at $\sqrt{s}=7 \mathrm{TeV}$. Lead-lead $(\mathrm{Pb}-\mathrm{Pb})$ collisions were recorded at $\sqrt{s}=2.76 \mathrm{TeV}$ in 2010 and 2011, at $\sqrt{s}=5.02 \mathrm{TeV}$ in 2015. Collisions at $\sqrt{s}=8 \mathrm{TeV}$ between protons (pp) were collected in 2012. In 2013 and in 2016, Proton-Lead (p-Pb) collisions were recorded at $\sqrt{s}=5.02 \mathrm{TeV}$ and $\sqrt{s}=8 \mathrm{TeV}$, respectively.

There are some specific points in the LHC ring where the two beams can interact. These are called interaction points, and ALICE in located in the interaction point 2. 


\section{CERN's accelerator complex}

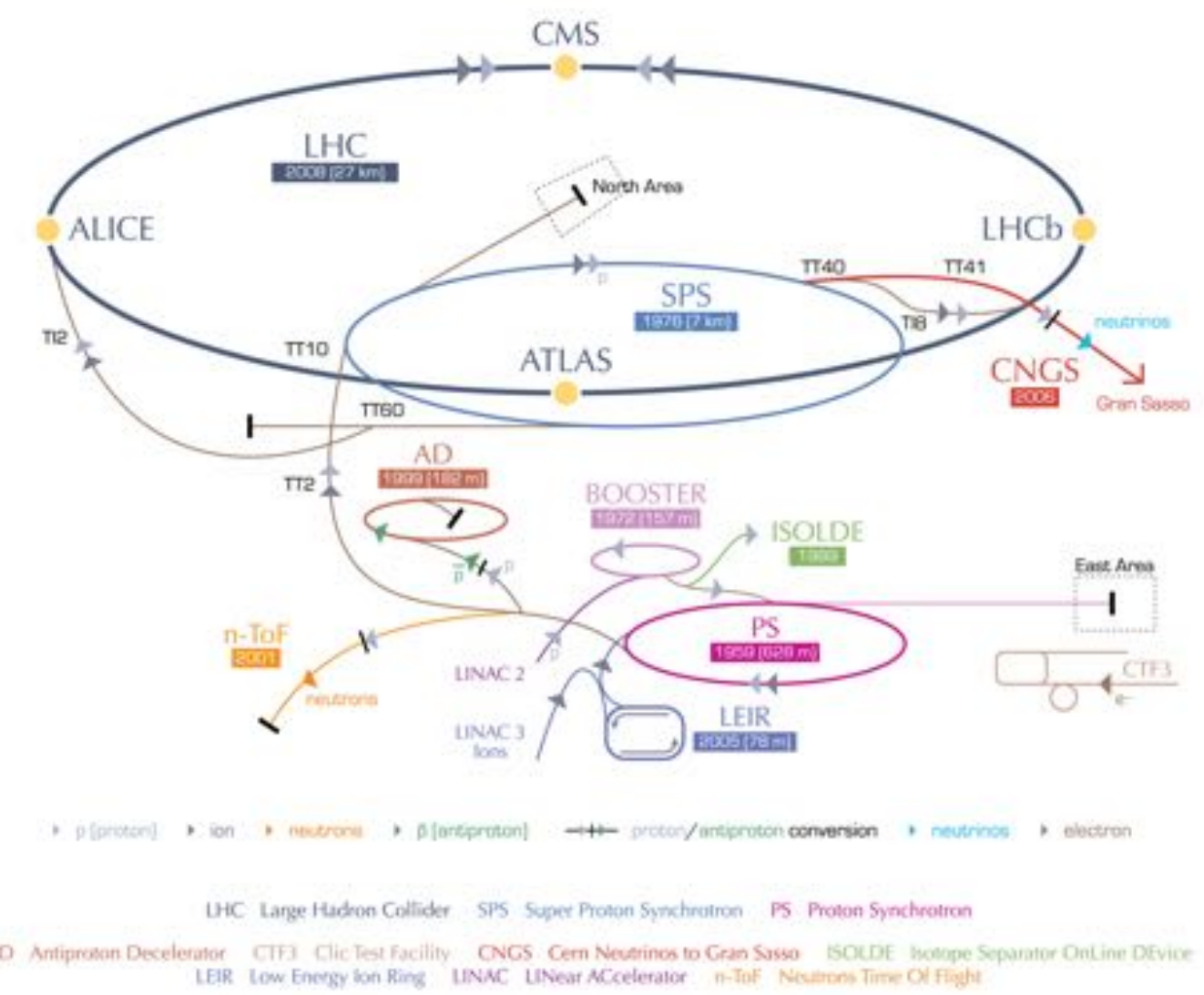

Figure 4.2: LHC complex scheme (adapted from [49]), where all the acceleration stages are visible.

\subsection{Inner Tracking System}

Table 4.1: ITS layers' geometry

\begin{tabular}{cccc}
\hline \hline Layer & Tecnology & radius $(\mathrm{cm})$ & $\min \mathrm{z} / \max \mathrm{z}$ \\
\hline 1 & Pixel & 3.9 & $-14.1 / 14.1$ \\
2 & Pixel & 7.6 & $-14.1 / 14.1$ \\
3 & Drift & 15.0 & $-22.2 / 22.2$ \\
4 & Drift & 23.9 & $-29.7 / 29.7$ \\
5 & Strip & 38.0 & $-43.1 / 43.1$ \\
6 & Strip & 43.0 & $-48.9 / 48.9$ \\
\hline \hline
\end{tabular}

The Inner Tracking System (ITS) is compose by six coaxial, cilindric layers surrounding the beam axis. It was projected to measure the interaction point of the collision (primary vertex) and also the vertices of some particle decays (secondary vertices), as well to improve the track reconstruction performed by the TPC.

The layers are located at different distances from the beam axis and have distinct technologies in them (table 4.1 summarizes this information). The main difference is 
their spatial precision: the inner, the more precise they are. This is done so the particle density, that is higher close to the interaction point than further from the collision region, does not affect much the measurement. Figure 4.3 shows a scheme of the detector layers.

- The first two layers are the Silicon Pixel Detector (SPD), the most precise;

- The following two are the Silicon Drift Detector (SDD);

- The most external two are the Silicon Strip Detector (SSD) (less precise).

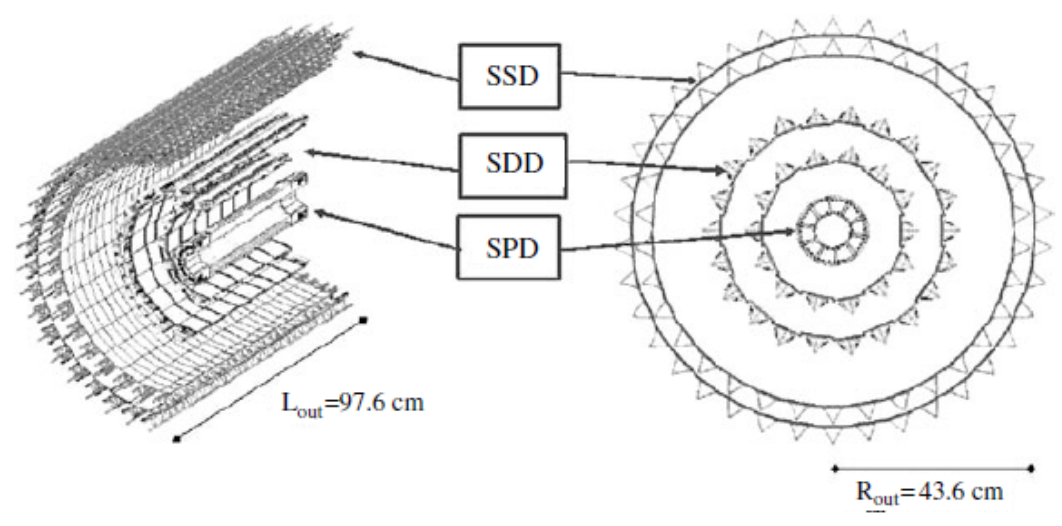

Figure 4.3: ITS Structure Scheme [44]: all the six layers are shown (SPD, SDD and SSD), as do their dimensions.

A very important use for the ITS is to select the particles created during the collision or close to the interaction point (primary particles). While primary particles have, in general, a hit in one of the ITS layers, the non-primary particles do not.

\subsection{Time Projection Chamber}

The Time Projection Chamber (TPC) is a gaseous detector that allows tracking and identification of particles through the measurement of their energy loss in the gas. It is a thick, cylindrical shell container whose inner radius is $\approx 85 \mathrm{~cm}$ and the outer one is $\approx 250 \mathrm{~cm}$. It is filled with a gaseous mixture of $\mathrm{CO}_{2} / \mathrm{Ne} / \mathrm{N}_{2}(10 \% / 85 \% / 5 \%)$ and it has three electrical plates, in both ends and one in the middle, that generate an electrostatic field parallel to the longitudinal axis, as shown in figure 4.4. In the cylinder ends, the readout is composed of 159 radial rows of trapezoidal pads, each row having a different number of them, making a total of more than 15.000 pads. When a particle passes through the gaseous mixture, ionizing it, the electrons that result from this interaction are driven by the electric forces to the detection pads. The collected charge allows for spatial and energy deposition information. 


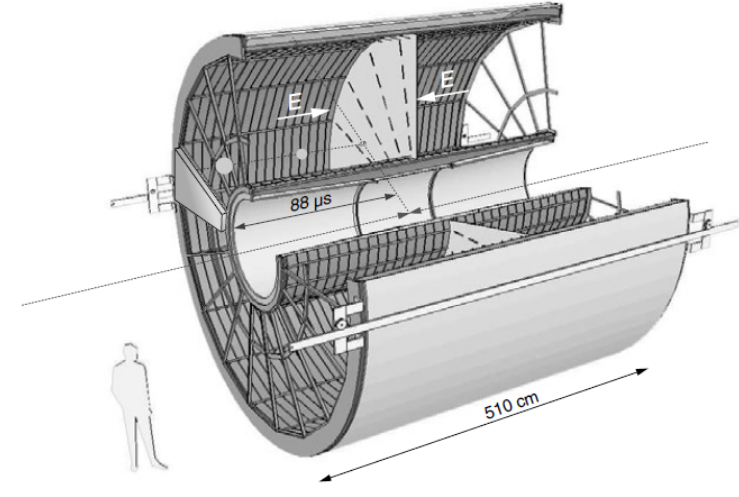

Figure 4.4: TPC scheme 44]. The gas cylindrical thick shell is visible, with its dimensions and the electric field direction. A human was drawn as a size reference.

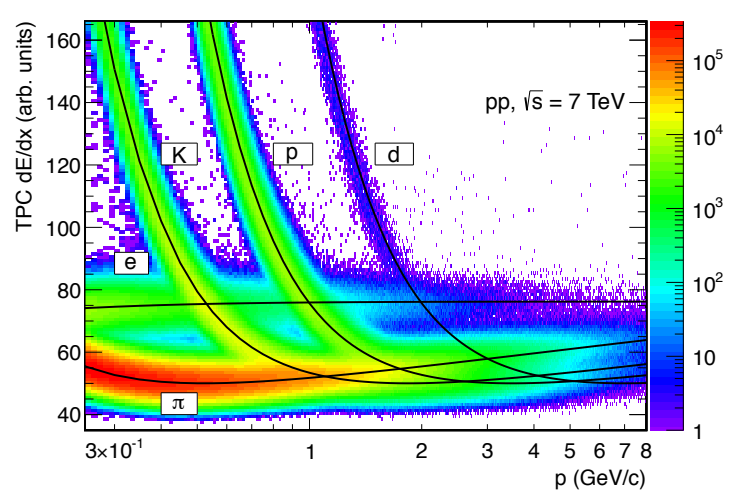

Figure 4.5: The TPC energy loss [50] curves for each particle. The black lines show the expected mean values for some species drawn over the ALICE pp at $\sqrt{s}=7 \mathrm{TeV}$ data.

The XY position of the track is easily associated to the pad's. Since the velocities of the particles from the collision is much greater than the one of the electrons from the ionization in the gas, one can calculate the position in the Z-axis from the time interval between the collision and the signal arrival in the pads. The position information is used, together with the position provided by the ITS and the electromagnetic field values, in a fitting algorithm to reconstruct the particle trajectory through the barrel and calculate its momentum.

The amplitude of the signal, i.e. the quantity of electrons that arrives at the pads, can be associated to the energy loss in the gases. The expected energy loss [51] $\langle d E / d x\rangle$ for each particle can be used to determine the particle species that generated each track, and can be parametrized by equation 4.1 .

$$
\langle d E / d x\rangle=\frac{C_{1}}{\beta^{2}}\left[\ln \left(C_{2} \beta^{2} \gamma^{2}\right)-\beta^{2}+C_{3}\right]
$$

where $C_{i}$ are detector specific constants, and $\beta$ and $\gamma$ are defined as in equation 4.2 .

$$
\beta=v / c, \quad \gamma=\frac{1}{\sqrt{1-\beta^{2}}}
$$

Figure 4.5 illustrates this procedure. An important variable for identification analysis is the $N_{\sigma, T P C}$ for a given species, as in equation 4.3. which is the measured minus the expected value of the energy loss for the species, normalized by the $\langle d E / d x\rangle$ measurement resolution.

$$
N_{\text {sigma }, T P C}=\frac{\langle d E / d x\rangle_{\text {measured }}-\langle d E / d x\rangle_{\text {species }}}{\sigma_{\text {species }}}
$$




\subsection{Electromagnetic Calorimeter}

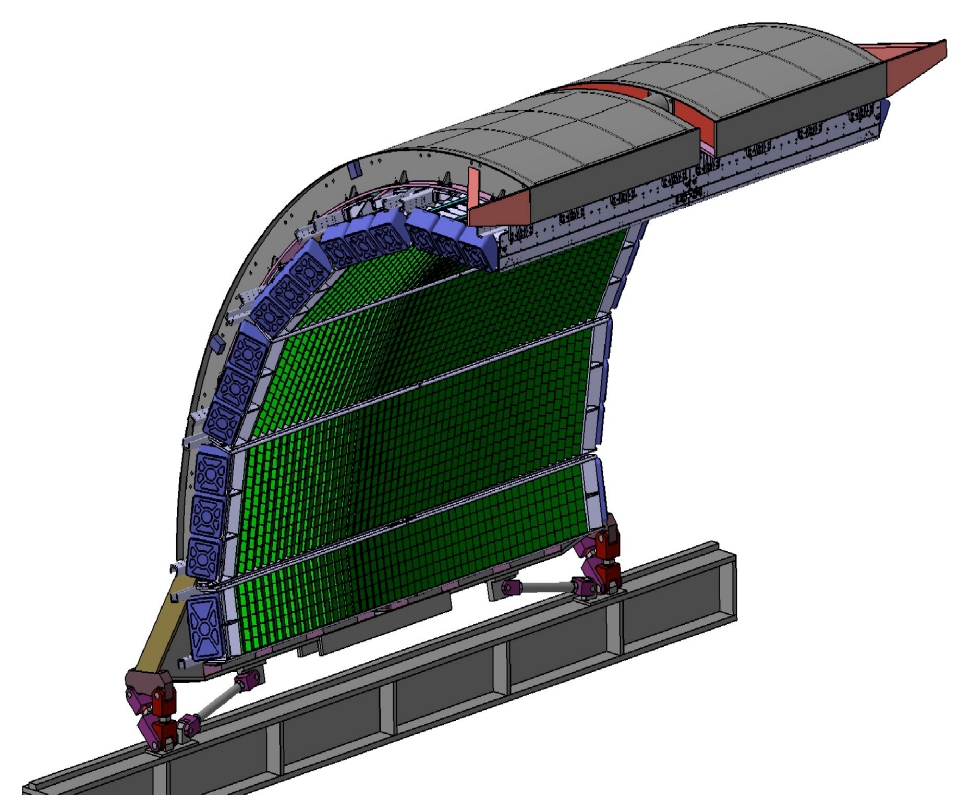

Figure 4.6: EMCal scheme [45], where one can see its $10+2 / 3$ modules in cylindric strucutre, covering approximately $100^{\circ}$.

The Electromagnetic Calorimeter (EMCal) was projected to detect the particles energies. Its basic component is a $6 \times 6 \mathrm{~cm}^{2}$ tower, which has 76 lead layers of $1.44 \mathrm{~mm}$ thick each and 77 scintilator layers of $1.76 \mathrm{~mm}$ thick each (Shashlik tecnology [45]). For this design, photons and electrons deposit its total energy, while hadrons typically deposit only a small fraction [45]. The readout is done by wavelength shifting fibres (WSF) placed perpendicularly to the lead-scintilator tiles.

The detector is composed of 12288 towers. Each group of $2 \times 2=4$ towers is clustered in a module. The set of $4 \times 24=288$ modules is a Trigger Region Unity (TRU) and 3 TRU compose a supermodule. There are ten complete supermodules and two thirds of a supermodule (in two columns of $5+\frac{1}{3}$ ), that covers $\Delta \varphi \approx 100^{0}$ and $-0.7<\eta<0.7$.

The EMCal main uses include:

- Measurement of the energy deposited by photons and electrons, including those coming from hadrons that decay through these particles;

- Trigger events with high transverse energy (Level-0 and Level-1);

- Electron identification via $E / p$ ratio. 


\subsection{V0}

V0 [52, 53 is an almost zero-degree detector, i.e. a detector close to the beam direction, that can be used to estimate the intensity of particle production of the collision. This means that one can infer from V0 signal whether there was a collision between hadrons, i.e. it can be used as a Minimum Bias (MB) trigger detector, that will be discussed in chapter 5 .

The detector is compose of two assymetric parts: V0A and V0C. Both are segmented discs, with four concentric rings and eight $\pi / 4$ sectors. V0A is in the forward region, at $340 \mathrm{~cm}$ from the nominal collision point. Its rings pseudorapidity interval are $[5.1,4.5,3.9,3.4,2.8]$. Similarly, V0C is in the backward region, at $-90 \mathrm{~cm}$ from the nominal collision point. Its rings pseudorapidity interval are $[-3.7,-3.2,-2.7,-2.2,-1.7]$. All $4 \times 8=32$ segments are visible in figure 4.7

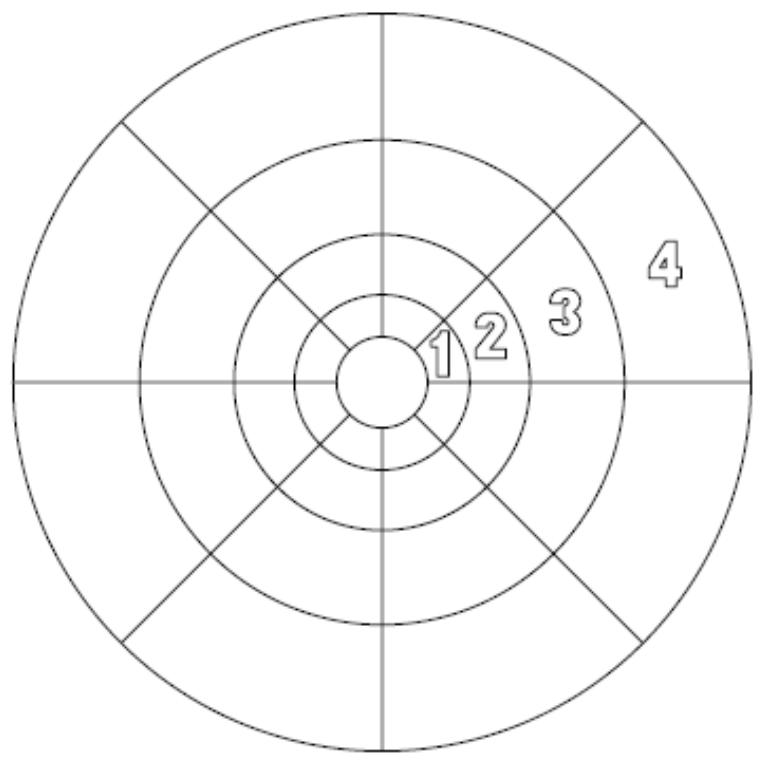

Figure 4.7: V0 Scheme, where one can see the 4 rings and 8 sectors [44. Each ring covers a different pseudorapidity region, as described in the text. 


\section{Data Analysis}

In this chapter, the general procedure of the analysis will be described. The event triggering and selection process is shown in section 5.1. The track reconstruction using TPC information, for both jet reconstruction and particle identification, is detailed in section 5.3. The selection of tracks that are used in the electron identification analysis, including TPC + EMCal information, and the main corrections to be applied, can be found in section 5.5 .

\subsection{Event Selection}

Due to the large amout of information produced in each collision, one has to select the events in order to save processing and analysing time. The triggering system is projected to do this selection and to record the events that are of interest. The first selection corresponds to the bunch crossing with interactions. A possible second selection corresponds to events that generate a signal in a specific detector, which can favour certain physical processes.

The trigger is the result of a specific detector or detectors response that allows all ALICE systems to measure and record an event. In the following sections, two of the triggers will be detailed: the Minimum Bias (MB) trigger, that is related to bunch crossing with collisions; and the EMCal $(E M C)$ trigger, that is related to events with a minimum energy deposition in the EMCal.

\section{Minimum Bias Trigger}

In order to reject noise-only events, the V0 detectors signals are used. When there is a collision, scattered particles reach both V0A and V0C detectors, but due to the different distances between V0A and V0C and the interaction point, the signals must differ by approxiamtely $8 \mathrm{~ns}$ (figure 5.1). This is not true for a bunch-residual gas interaction, which can generate signal in both V0 detectors any time that one of the bunches of the beam passes the ALICE detectors. Hence, both detectors must measure some activity inside time windows, clearly visible in figure 5.2 [53] $t_{V 0 A}=t_{0}+11$ ns and $t_{V 0 C}=t_{0}+3$ $\mathrm{ns}$, where $t_{0}$ is the instant of a bunch crossing. If these conditions are satisfied, the event 
is tagged as Minimum Bias (MB).

V0 detector signal can be combined with SPD signal, since both cover different acceptance regions in $\eta$, in order to select $\mathrm{MB}$ events. In this case, besides the coincident V0 signals, there must be at least one hit in each layer of the SPD (first two layers of ITS).

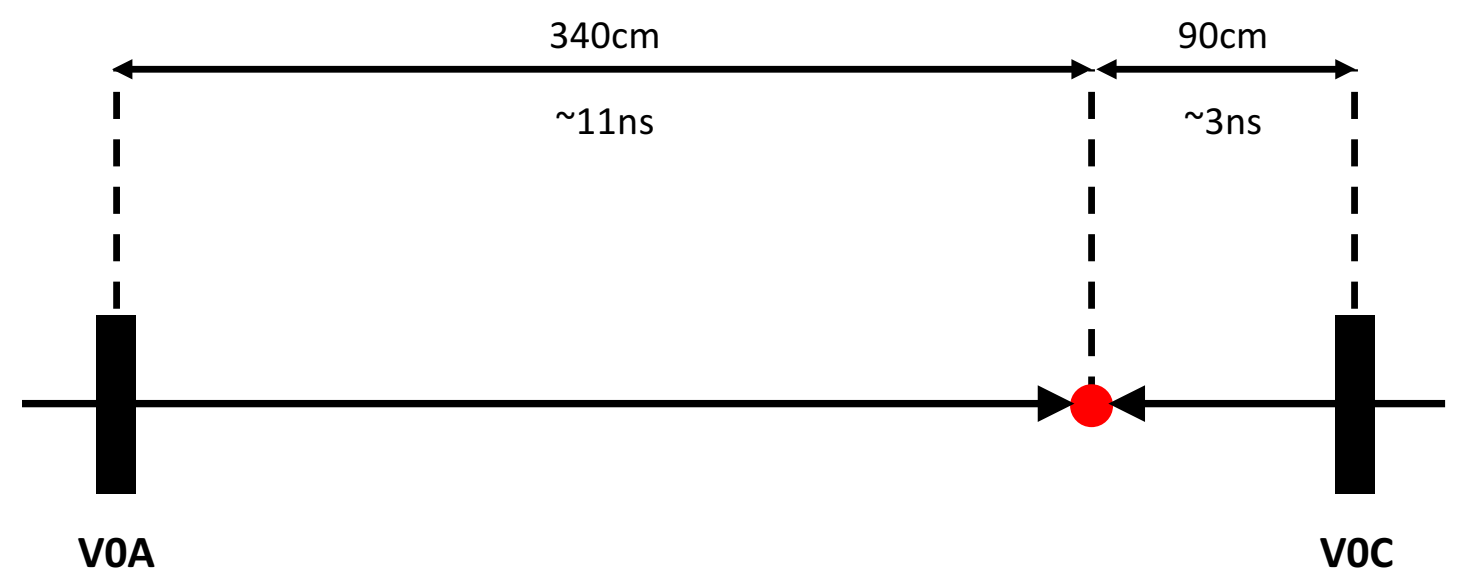

Figure 5.1: V0 in MB Trigger Scheme: the different distances of V0A and V0C are taken into account for triggering, i.e. deciding to record the event.

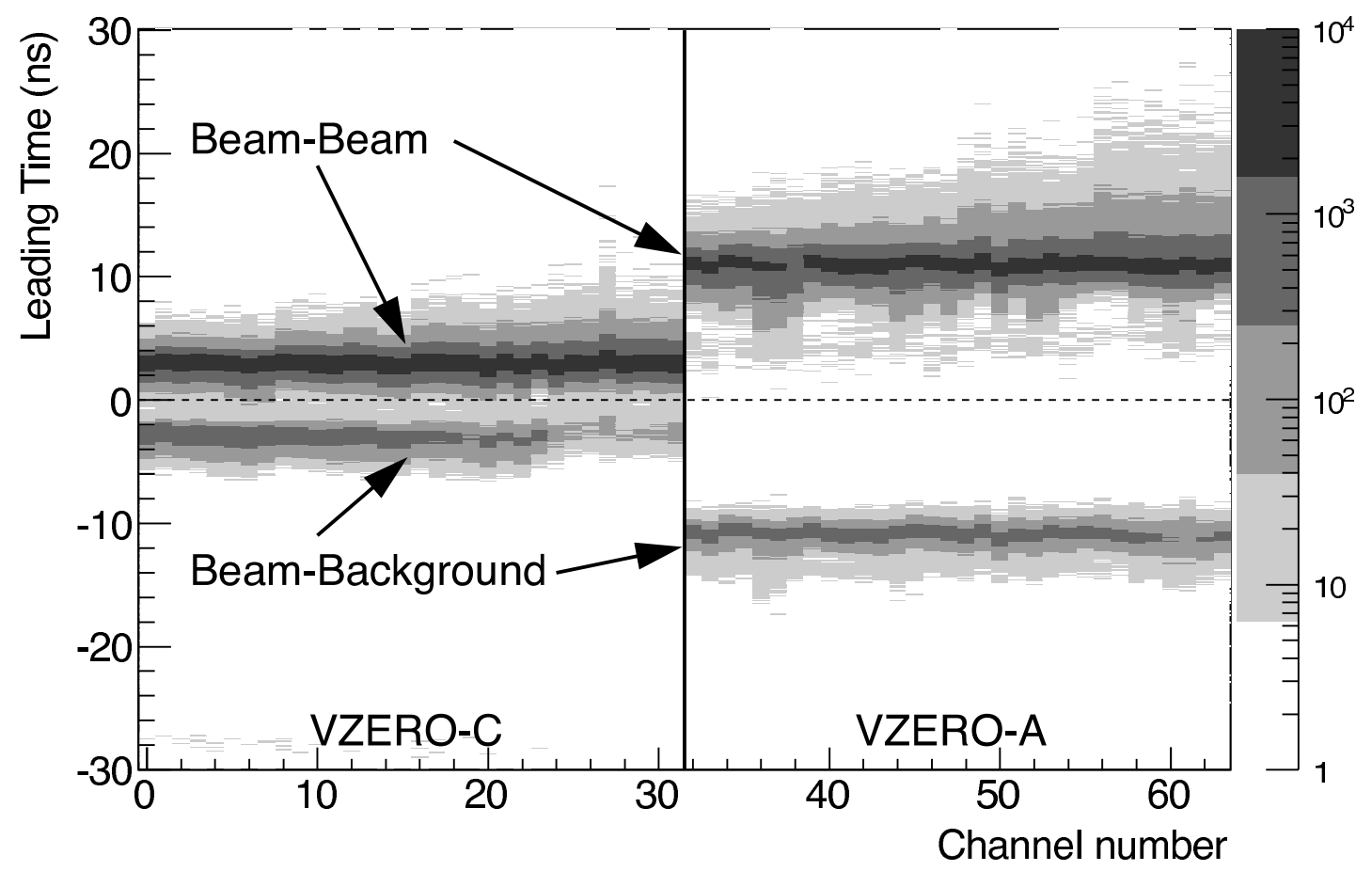

Figure 5.2: V0 times in MB trigger [53]. The beam-gas interaction signals are highlighted as beam-background. The beam-beam signal are around the time difference expected because of the different distances from the interaction point. 


\section{EMCal Triggers}

The EMCal is used as a triggering detector in two ways:

- Level-0 (L0): The trigger calculation are done by hardware only;

- Level-1 (L1): The trigger calculation are done by hardware and software;

The L0 is known as EMC7 trigger and the two L1 triggers are EMCEGA and EMCEJE [45, 51].

- L0 - EMC7: At this level, the energy of a patch, i.e. a $2 \times 2$-module region, is summed and compared to a threshold value $(\approx 2 \mathrm{GeV})$. The patches cannot be split between two TRU. If the energy is above the threshold, the event is tagged as EMC7.

- L1 - EMCEGA: At this level, the energy of $2 \times 2$-modules patch is summed and compared to a V0-dependent threshold value $(\approx 7$ or $\approx 11 \mathrm{GeV})$. The patches can be split between two TRU. If the energy is above the threshold, the event is tagged as EMCEGA.

- L1 - EMCEJE: At this level, the energy of $4 \times 4$-module patch is summed and compared to a V0-dependent threshold value $(\approx 20 \mathrm{GeV})$. The patches can be split between two TRU. If the energy is above the threshold, the event is tagged as EMCEJE.

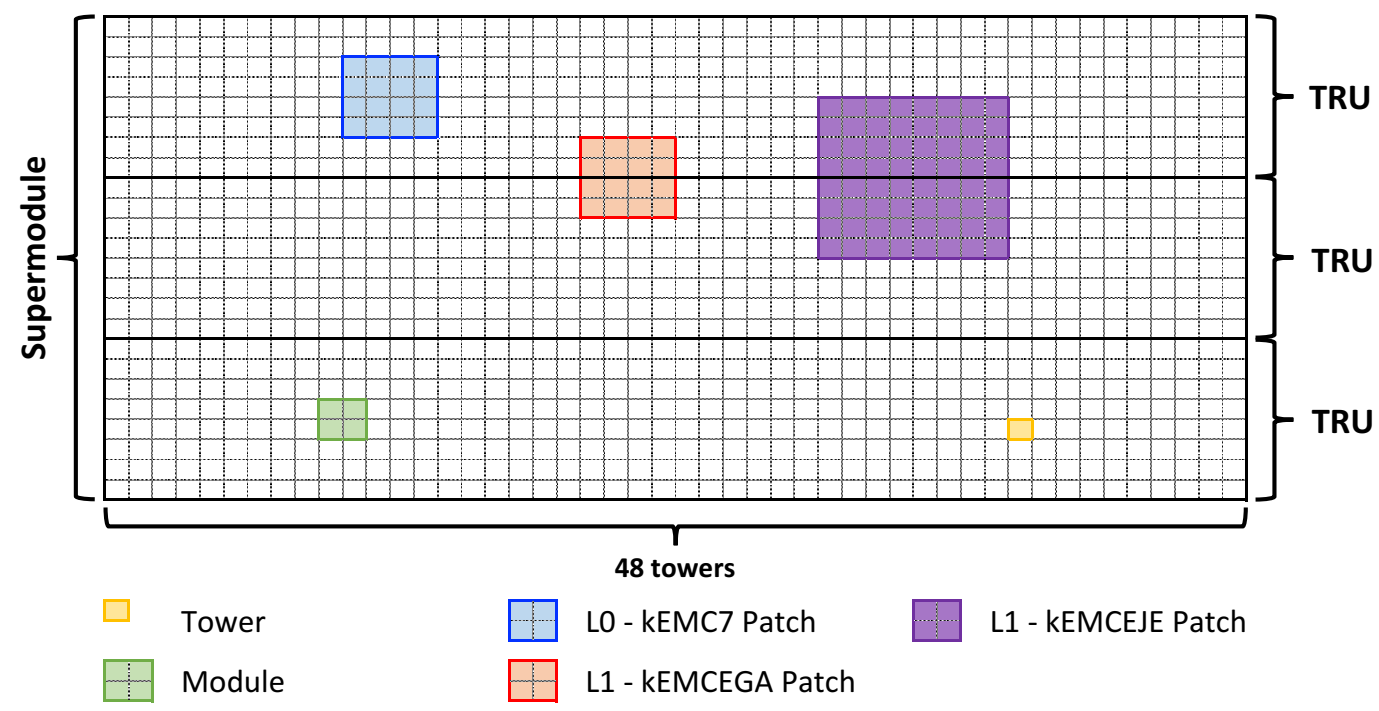

Figure 5.3: EMCal trigger patches for different triggers, represented in a supermodule scheme: in blue, is the patch of the L0 trigger, that must be inside a TRU; in red, is the L1-kEMCEGA trigger patch, that may be in two different TRUs; the purple one is the L1-kEMCEJE trigger patch, that may be in two different TRUs.

Figure 5.3 shows the EMCal patches for each trigger. In this analysis, only kEMC7 events were used. 


\subsection{Cluster Reconstruction}

As the particles pass through the EMCal layers, they produce photons that convert into electron-positron pairs, which also produce more photons. This process, known as electromagnetic shower, can spread to adjacent towers that must be grouped into clusters.

The clusterization of a shower is similar to the jet clusterization shown in section 3.3 . The used clusterizer V1 [45] is a simple geometrical algorithm (figure 5.4 shows it in a schematic way):

- the towers are listed in the EMCal internal identification order, i.e. number of the row and number of the column;

- the first one with energy greater than $100 \mathrm{MeV}$ is chosen as a seed;

- the seed's (first) neighbours are identified as the towers in the next and previous line, or in the next and previous column;

- the neighbours are included in the cluster with the seed if its energy is greater than $10 \mathrm{MeV}$;

- the procedure is repeated until no neighbour can be added to the cluster.

- the next seed is the tower with energy greater than $100 \mathrm{MeV}$ that has not been clusterized yet;

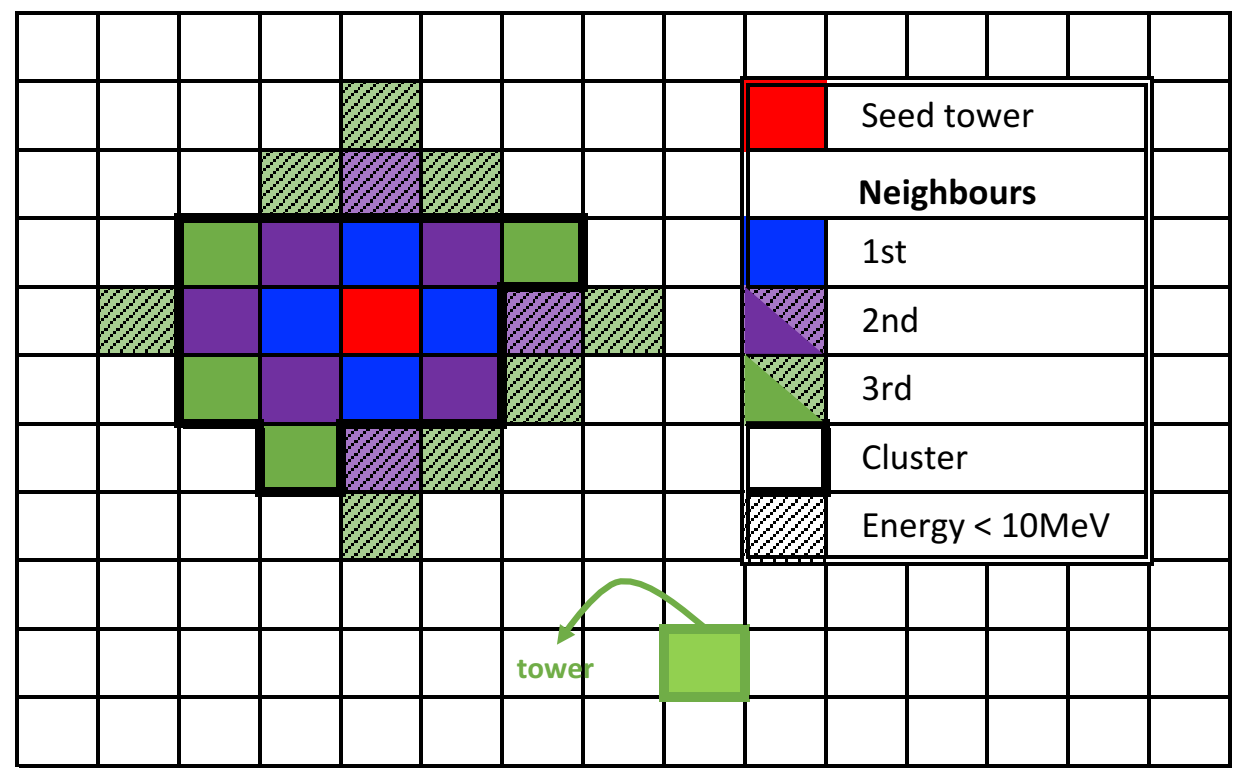

Figure 5.4: The cluster reconstruction, according to the clusterizer V1, pictoricaly drawn for EMCal towers. 
The recombination scheme (as detailed in 3.3) for merging towers in one cluster (equations 5.1 and 5.2] is a weighted $p_{T}$-scheme [54, 51]: the cluster position is a weighted sum of the tower's position; the cluster energy is simply the energy sum.

$$
\begin{gathered}
E_{\text {cluster }}=\sum_{i} E_{i} \\
\omega_{i}=\max \left\{0, \omega_{0}+\ln \left(E_{i} / E_{T}\right)\right\} \\
\left\langle\varphi_{\text {cluster }}\right\rangle=\sum_{i} \frac{\omega_{i} \varphi_{i}}{\sum_{j} \omega_{j}},\left\langle\eta_{\text {cluster }}\right\rangle=\sum_{i} \frac{\omega_{i} \eta_{i}}{\sum_{j} \omega_{j}} \\
\left\langle\varphi^{2}\right\rangle=\sum_{i} \frac{\omega_{i} \varphi_{i}^{2}}{\sum_{j} \omega_{j}}, \quad\left\langle\eta^{2}\right\rangle=\sum_{i} \frac{\omega_{i} \eta_{i}^{2}}{\sum_{j} \omega_{j}}, \quad\langle\eta \varphi\rangle=\sum_{i} \frac{\omega_{i} \eta_{i} \varphi_{i}}{\sum_{j} \omega_{j}}
\end{gathered}
$$

The cluster shape is approximated to an ellipse (figure 5.5). The semi-minor and semimajor axes can be calculated using the variables in equation 5.3, known as shower shape variables, as shown in equations 5.4 and 5.5 [54, 55]. Both M02 and M20 distributions are different depending on the particle species that generated the signal. Therefore, they can be used in particle identification. In this analysis, $M 20<0.3$ selection was used due to its improvement to the electron identification [56].

$$
\begin{gathered}
\sigma_{\varphi^{2}}=\left\langle\varphi^{2}\right\rangle-\left\langle\varphi_{\text {cluster }}\right\rangle \\
\sigma_{\eta^{2}}=\left\langle\eta^{2}\right\rangle-\left\langle\eta_{\text {cluster }}\right\rangle \\
\sigma_{\eta \varphi}=\langle\eta \varphi\rangle-\left\langle\eta_{\text {cluster }}\right\rangle\left\langle\varphi_{\text {cluster }}\right\rangle \\
M 02 \equiv \lambda_{0}^{2}=\frac{\left\langle\eta_{\text {cluster }}\right\rangle^{2}+\left\langle\varphi_{\text {cluster }}\right\rangle^{2}}{2}+\sqrt{\frac{1}{4}\left(\sigma_{\eta^{2}}-\sigma_{\varphi^{2}}\right)^{2}+\sigma_{\eta \varphi}^{2}} \\
M 20 \equiv \lambda_{1}^{2}=\frac{\left\langle\eta_{\text {cluster }}\right\rangle^{2}+\left\langle\varphi_{\text {cluster }}\right\rangle^{2}}{2}-\sqrt{\frac{1}{4}\left(\sigma_{\eta^{2}}-\sigma_{\varphi^{2}}\right)^{2}+\sigma_{\eta \varphi}^{2}}
\end{gathered}
$$




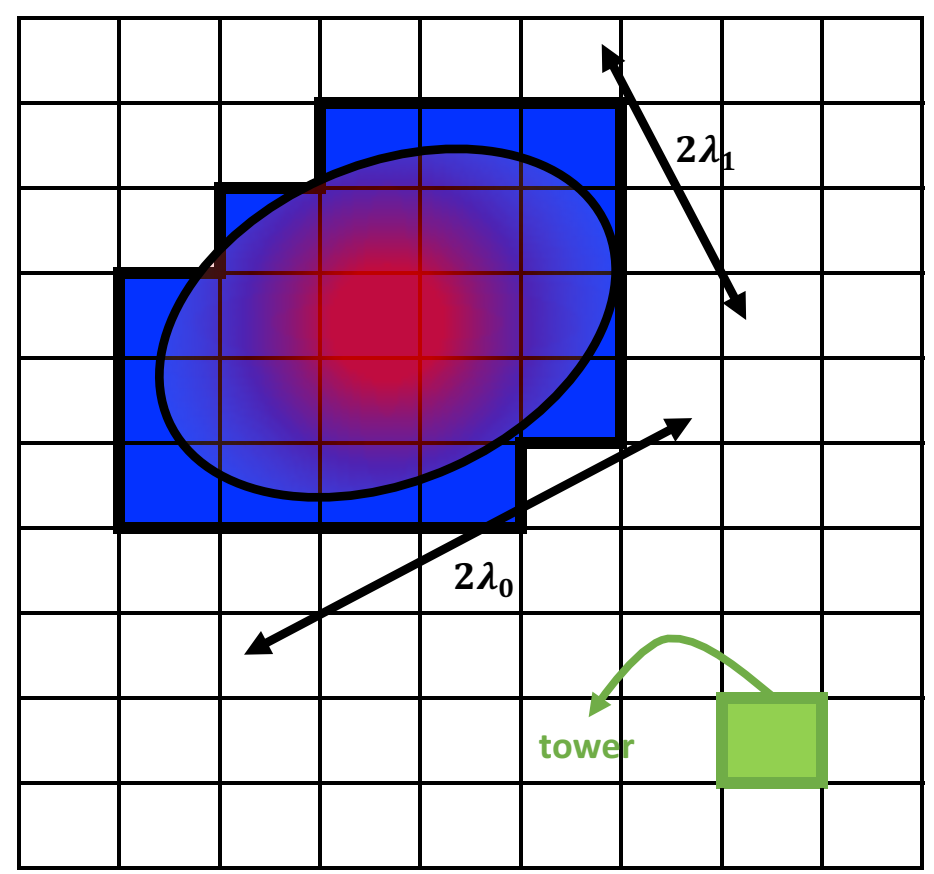

Figure 5.5: EMCal shower shape variables from an ellipse approach. $\lambda_{0}$ and $\lambda_{1}$ formulas are in the text.

\subsection{Track Reconstruction}

As described in chapter 4, the detectors in the central barrel region, i.e. ITS, TPC, EMCal, etc, are immerse in a longitudinal, 0.5 T-magnetic field that causes the bending of the charged particles trajectories.

The charged particles ionize the gas through their path, producing electrons that induce charge in the TPC pads. The projection of the trajectories in the transverse $x \times y$ plane is obtained from the combination of the pads positions. The trajectory projection in the longitudinal direction ( $z$ axis) is obtained from the time it takes for the signal to reach the readout chambers. This can be done because the speed of the particles produced in the collision is much greater than the ionized electrons drift speed in the gas: the whole particle path is ionized almost at the same time, so any different position in $z$ can be accounted as the drift speed times the duration of the drift.

The clustering algorithm for the tracks combines five pads in the same row (radius bin) and five bins in the $z$ direction (time bins), around the maximum signal amplitude [51. The cluster position is the center of gravity of these $5+5$ bins, and it is associated to a point of the particle trajectory. The clusters must be combined in order to reconstruct a track. In addition, the ITS hits are added to this set of clusters and are combined based in a Kalman filter algorithm [44, 46].

The tracks can be parameterized by a helix function [57, 58] (figure 5.6) from which the charge and momentum of the particles can be retrieved. A helix is fitted to the TPC clusters according to equation 5.6 , 


$$
\begin{aligned}
& x(s)=x_{0}+\frac{1}{k}\left[\cos \left(\Phi_{0}+h s k \cdot \cos \lambda\right)-\cos \Phi_{0}\right] \\
& y(s)=y_{0}+\frac{1}{k}\left[\sin \left(\Phi_{0}+h s k \cdot \cos \lambda\right)-\sin \Phi_{0}\right] \\
& z(s)=z_{0}+s \cdot \sin \lambda
\end{aligned}
$$

where $x_{0}, y_{0}, z_{0}$ are the first point of the track; $k=1 / R$ is the helix curvature $(R$ is the helix radius); $\lambda$ is the dip angle; $h= \pm 1$ is the sense of the rotation around the z-axis (related to the particle charge); and $\Psi_{0}=\Psi-h \pi / 2$, where $\Psi$ is the azimutal angle of the track in the initial position.

From the parameters, one can calculate the kinematic variables, in $\mathrm{GeV} / c$ as following:

$$
\begin{aligned}
p_{T} & =c q B / k \\
\tan \lambda & =p_{T} / p_{z} \\
p & =\sqrt{p_{T}^{2}+p_{z}^{2}}
\end{aligned}
$$

where $c$ is the speed of light in $[\mathrm{m} / \mathrm{ns}]$; $\mathrm{q}$ is the electric charge (in multiples of $e$ ); $B$ is the magnetic field in $[\mathrm{T}]$.

After the track is reconstructed, it can be associated to possible EMCal clusters, whose reconstruction was discussed in section 5.2. The trajectory is extrapolated until the EMCal surface and a geometrical condition for $\eta \times \varphi$ is used as a matching parameter: if $\left\|\eta^{\text {cluster }}-\eta^{\text {track }}\right\|<0.05$ and $\left\|\varphi^{\text {cluster }}-\varphi^{\text {track }}\right\|<0.05$, track and cluster are matched and associated.

\section{Tracks in Jets}

After the reconstruction, the tracks still have to be selected according to the needs of the analysis. For the charged jet reconstruction, a uniform distribution of tracks (charged particles) is needed, so the jets are not geometrically biased. Therefore, only the tracks inside the TPC acceptance $[-0.9,0.9]$ are used.

Table 5.1 summarizes the selection criteria applied to tracks used in the jet reconstruction. All the jets in this analysis are charged jets.

Table 5.1: Tracks used in charged jet reconstruction

\begin{tabular}{cc}
\hline \hline Variable & Cut \\
\hline$p_{\mathrm{T}}$ & $>0.15 \mathrm{GeV} / c$ \\
$\eta$ & $\in[-0.9,0.9]$ \\
\hline \hline
\end{tabular}




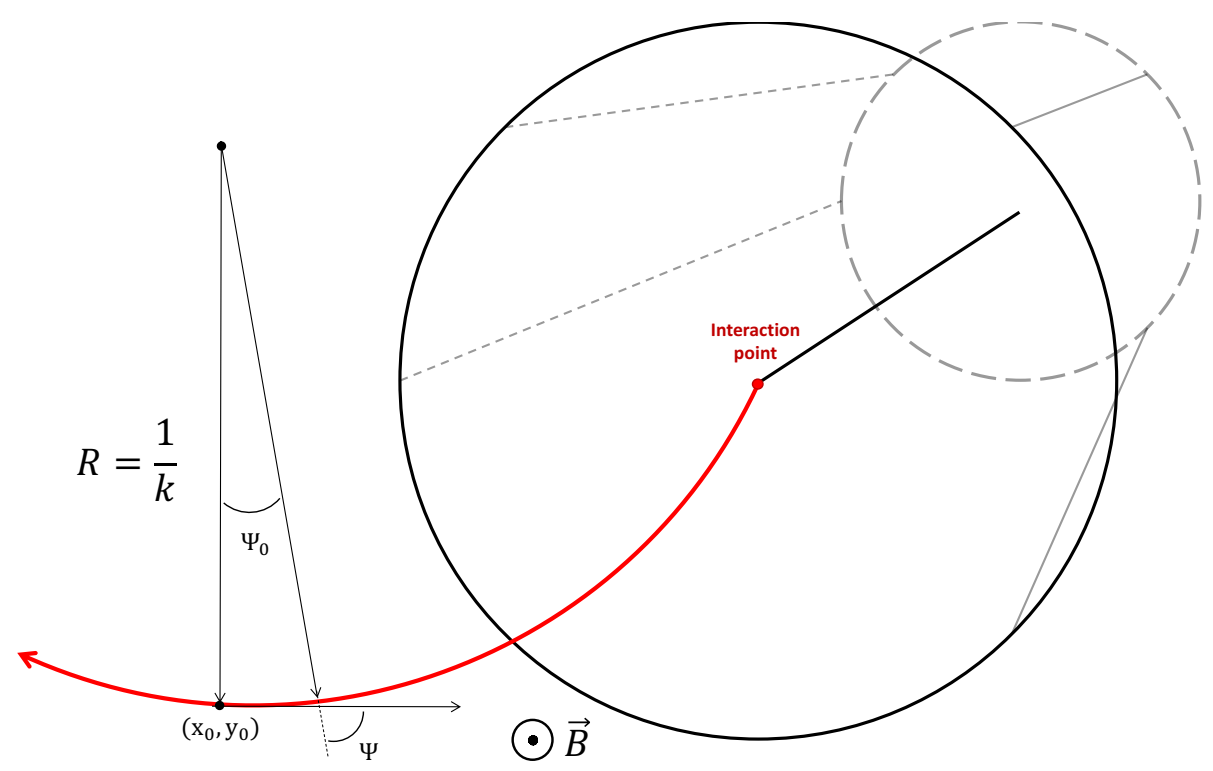

Figure 5.6: Track helix reconstruction scheme, where the variables defined in the text are drawn. The cylinder represents the TPC region and the red line the clusterized TPC points. In general, tracks have its origin close to the interaction point, as drawn in the figure.

\section{Tracks for Particle Identification}

Since the analysis requires a heavy-flavour decay electron and the chosen electron identification method uses energy information, these tracks must have a cluster in the EMCal. Table 5.2 summarizes the selection criteria applied to them. This set of tracks is the one used in particle identification.

Table 5.2: Tracks used in particle identification

\begin{tabular}{cc}
\hline \hline Variable & Cut \\
\hline DCA $(x \times y$ plane $)$ & $<2.4 \mathrm{~cm}$ \\
DCA $(z$ direction $)$ & $<3.2 \mathrm{~cm}$ \\
ITS Clusters & $>1 \mathrm{Hit}$ \\
$p_{\mathrm{T}}$ & $>0.15 \mathrm{GeV} / c$ \\
$\eta$ & $\in[-0.7,0.7]$ \\
TPC Clusters & $>70$ \\
$\mathrm{M} 20$ & $<0.3$ \\
$E / p$ & $\in[0.0,1.5]$ \\
\hline \hline
\end{tabular}

\subsection{Jet Reconstruction}

Jets are reconstructed using the Fastjet package [8]. The signal jets, i.e. the ones that will be used in the analysis and have physical meaning, where reconstructed by the anti $-k T$ algorithm, with parameter $R=0.4$, and the $p_{\mathrm{T}}$-scheme for the recombination. 
These jet sets may include some background jets, i.e. jets whose components are at least partially background. This is relevant only in $\mathrm{p}-\mathrm{Pb}$ collisions, where the particle density is higher, and so is the background. In order to correct this contribution, one can calculate an average background density $\rho$ and subtract it according to the jet area.

\section{Jet Area}

Fastjet uses a ghost technique [59] to calculate the jet area. Ghosts are defined as extremely low momentum fake particles, that are added to the event. Each of them has an area equivalence of $\Delta \eta \times \Delta \varphi=0.05=A_{\text {ghost }}$ and their position is such that the density of ghosts is uniform.

After the jet reconstruction, each jet will have a number of ghosts inside them. The jet area is taken as the number of ghosts $N_{\text {ghosts }}$ times the ghost area (equation 5.8). Jets with an area less than $0.6 \cdot \pi R^{2}$ were excluded from this analysis.

$$
A_{\text {jet }}=N_{\text {ghosts }} \times A_{\text {ghost }}
$$

\section{Background Density $\rho$}

The background density is calculated for $\mathrm{p}-\mathrm{Pb}$ events only, in a different procedure [60] when compared to the one widely used in $\mathrm{Pb}-\mathrm{Pb}$. The $p_{\mathrm{T}, \text { chjet }}$ density is taken as the median of the distribution of jet $p_{\mathrm{T} \text {,chjet }}$ over $A_{\text {jet }}$ ratio in the event, excluding the two most energetic ones. This density median $\left\{\frac{p_{\mathrm{T}, \text { chjet }}}{A_{\text {jet }}}\right\}$ is multiplied by an occupancy factor $C$, as shown in equation 5.9 .

$$
\rho_{p P b}=\operatorname{median}\left\{\frac{p_{\mathrm{T}, \mathrm{chjet}}}{A_{\text {jet }}}\right\} \cdot C
$$

where $C$ is the ratio between the area occupied by all physical (at least one track that is not a ghost) and the total phase space (ghosts area) (equation 5.10).

$$
C=\frac{\sum_{\text {phys.jets }} A_{\text {jet }}}{A_{\text {total }}}
$$

The corrected jet $p_{\mathrm{T}, \text { chjet }}^{\text {corr. }}$ is calculated according to equation 5.11 .

$$
p_{\mathrm{T}, \mathrm{chjet}}^{\text {corr }}=p_{\mathrm{T}, \mathrm{chjet}}-\rho_{p P b} \times A_{\text {jet }}
$$

\subsection{Electron Identification}

The set of tracks for particle identification (table 5.2) is used in the electron identification procedure. In the following sections, the heavy-flavour decay electrons identification 
procedure details will be described: TPC energy loss identification; $E / p$ selection and hadronic contamination; and non-heavy-flavour electron (non-HFe)contamination.

\section{TPC Identification}

In order to select electrons, one can constrain the tracks $N_{\sigma T P C}^{e}$, by defining a interval of acceptance. Due to the overlap of the hadron $d E / d x$ band to the electron one, the chosen non-symmetric range $-1<N_{\sigma T P C}^{e}<3$ results in a purer electron sample (figure 5.7).

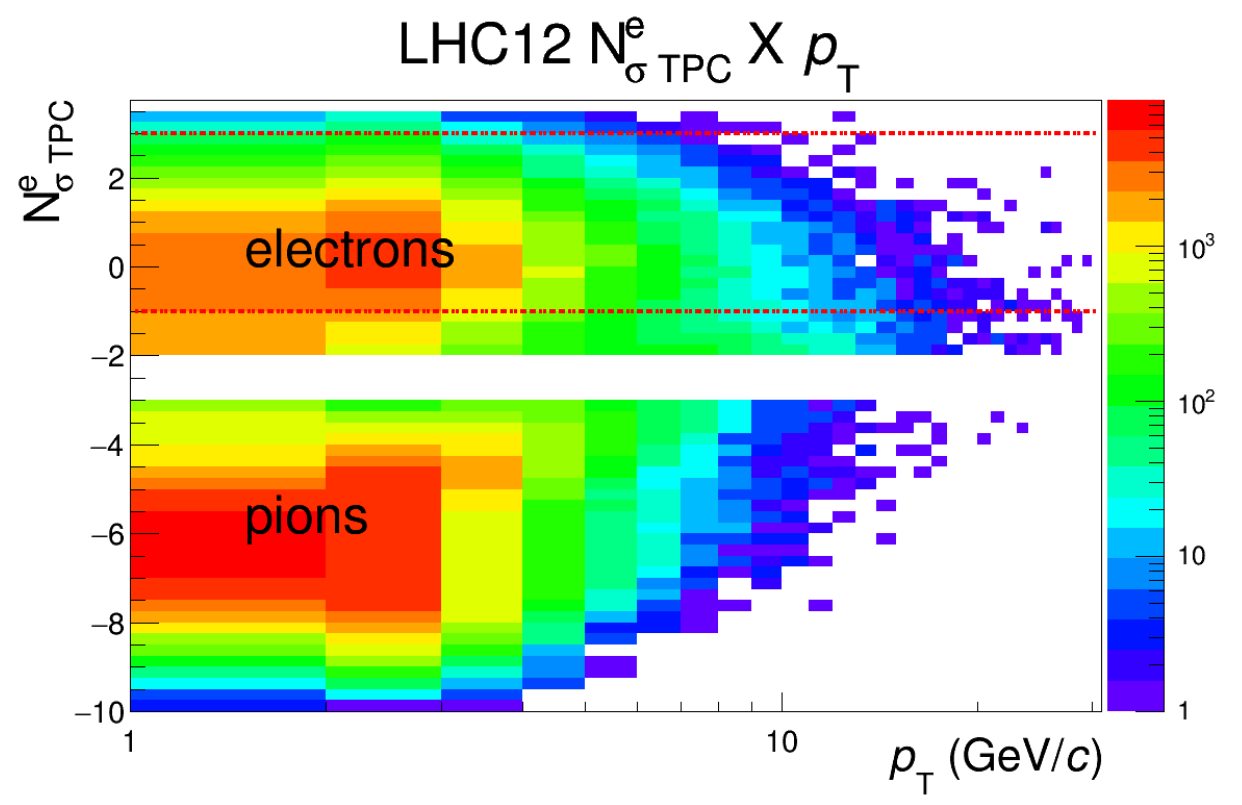

Figure 5.7: TPC energy loss distribution $\left(N_{\sigma T P C}^{e}\right)$ as fuction of transversal momentum $p_{T}^{e}$. The empty region delimitates two different statistics regions (see appendix A.3): the bottom part has $80 \%$ of its total statistics discarded randomly, while the top one does not. The electron and the pion region are highlighted. The data are from pp collisions, taken in 2012 by ALICE.

\section{$E / p$ selection}

The hadron-electron overlap must be statistically estimated so one can correct for misidentified particles (hadrons as electrons). This can be done by using the energymomentum ratio $E / p$ of the particles. Electrons deposit almost their whole energy in the EMCal and, thus, due to their small mass, will often produce a distribution $E / p \approx 1$. This is not true for hadrons, that do not deposit a great fraction of their energy in the EMCal.

In order to improve the EMCal selection, one can apply a cut in the M20 parameter. For M20 < 0.4, there is an electron dominance and, thus, a better purity in the sample.

One can assume that the shape of the $E / p$ distribution does not depend on $N_{\sigma T P C}$, i.e. misidentified pions and correctly identified pions produce an equivalent $E / p$ curve. 
This means that an electron TPC selection leads to a $E / p$ distribution with two contributions, according to equation 5.12 , where the misidentified hadrons distribution $E / p^{h}$ can be substituted by a distribution for correctly identified hadrons $\left(-10<N_{\sigma T P C}^{e}<-3\right)$ renormalized by a factor $f$.

$$
\begin{aligned}
& E / p\left(-1<N_{\sigma T P C}^{e}<3\right)=E / p^{e}\left(-1<N_{\sigma T P C}^{e}<3\right)+E / p^{h}\left(-1<N_{\sigma T P C}^{e}<3\right) \\
& E / p\left(-1<N_{\sigma T P C}^{e}<3\right)=E / p^{e}\left(-1<N_{\sigma T P C}^{e}<3\right)+f \cdot E / p\left(-10<N_{\sigma T P C}^{e}<-3\right)
\end{aligned}
$$

The factor $f$ can be calculated so that the $E / p\left(-1<N_{\sigma T P C}^{e}<3\right)$ and $E / p\left(-10<N_{\sigma T P C}^{e}<-3\right)$ distributions have the same integral in the range where they are expected to have only hadrons, i.e. for low $E / p$ values.

\section{Non-heavy-flavour electron contamination}

After the identification procedure, the set of remaining tracks is a highly pure electron sample. However, one still needs to estimate the fraction of non-heavy-flavour electrons in this sample. The relevant sources of non-heavy-flavour electrons (non-HFe) are photonic conversions in the detector materials, i.e. electron-positron pair production, and neutral hadrons decays (like pions Dalitz decay that can result in $2 e^{+} e^{-}$pairs). In any case, the invariant mass of the pair has as upper limit the mass of the mother particle, mainly photons $\left(m_{\gamma}=0 \mathrm{GeV}\right)$ and pions $\left(m_{\pi} \approx 0.135 \mathrm{GeV}\right)$, that can be used as a selection criterion for these contributions.

The selected electron tracks are combined to other electron tracks, that are called partner electrons. The partners are selected with looser cuts in order to obtain a statistically valid invariant mass distribution. The partner cuts are disposed in table 5.3 .

Table 5.3: Tracks used in Invariant Mass Method

\begin{tabular}{cc}
\hline \hline Variable & Cut \\
\hline$p_{\mathrm{T}}$ & $>0.15 \mathrm{GeV} / c$ \\
$\eta$ & $\in[-0.9,0.9]$ \\
$N_{\sigma T P C}^{e}$ & $\in[-3,3]$ \\
\hline \hline
\end{tabular}

One can calculate the e-jet spectra $(J)$ associated to partner electrons of the same charge (electron-electron or positron-positron) $J_{L S}$ and oposite charges (electron-positron) $J_{U L S}$. For an invariant mass cut, both have similar background contributions, since they are charge-independent, combinatorial-only: $J_{X, H F e-C o m b .}^{m<C u t}$ and $J_{X, C o m b-C o m b .}^{m<C u t}$, where $X$ is either like-sign $L S$ or unlike-sign $U L S$. The difference $J_{U L S}^{m<C u t}-J_{L S}^{m<C u t}$ would include physical sources only: same or opposite charge electron pair physical production $J_{L S, P h y s}^{m<C u t}$ and $J_{U L S, P h y s}^{m<C u t}$. LS and ULS, both from low invariant mass particles. Since the latter is neglible, one can use the subtraction as the non-HFe contribution $J^{c o n v}$. (equation 5.13). 


$$
\begin{gathered}
J_{U L S}^{m<C u t}=J_{U L S, P h y s .}^{m<C u t}+J_{U L S, H F e-C o m b .}^{m<C u t}+J_{U L S, \text { Comb. }-C o m b .}^{m<C u t} . \\
J_{L S}^{m<C u t}=J_{L S, \text { Phys. }}^{m<C u t}+J_{L S, H F e-C o m b .}^{m<C u t}+J_{L S, \text { Comb. }-C o m b .}^{m<C u t} \\
J^{\text {conv. }}=J_{U L S, \text { Phys. }}^{m<C u t}=J_{U L S}^{m<C u t}\left(p_{\mathrm{T}}^{\text {track }}\right)-J_{L S}^{m<C u t}\left(p_{\mathrm{T}}^{\text {track }}\right)
\end{gathered}
$$

All the details and the peculiarities of the analysis will be presented in chapter 6 , for pp data, and in chapter 7, for p-Pb. There, the detection and method efficiencies will be explained, and the final observables and results will be shown. 


\section{Analysis in Proton-Proton Data}

In this chapter, the procedure for the analysis of the pp data will be described in details. In section 6.1, the event selection criteria is discussed. Section 6.3 describes the electron identification and selection of inclusive e-jet pairs. Sections 6.4 and 6.5 describe the background estimation and subtraction. Section 6.6 details the efficiency calculation for the electron selection. The unfolding technique procedure is discussed in section 6.8. The final observables are in section 6.9 and the systematic errors are shown in section 6.10 .

\subsection{Event Selection}

The proton-proton data used in this analysis ( $\mathrm{LHC12c}, \mathrm{d}, \mathrm{e}, \mathrm{f}, \mathrm{g}, \mathrm{h}, \mathrm{i}$ periods, whose run numbers are in appendix A.2 were recorded by ALICE in 2012, at $\sqrt{s}=8 \mathrm{TeV}$. Since the chosen electron identification method uses EMCal energy information, and requiring EMCal to have triggered the event increases the probability of electrons being detected, $E M C 7$ triggered events were selected. Only events with primary vertex position $\left|z_{v t x}\right|<$ $10 \mathrm{~cm}$ were used in this analysis in order to optimize the track reconstruction.

\subsection{Electron-Jet Pairs Selection}

The pre-selected tracks (cuts shown in table 5.2 are paired to all the jets in the event, so the electron identification procedure is applied to particle-jet pairs. They are kept separated in different sets that correspond to different $p_{\mathrm{T}}^{e}$ ranges $I_{p_{\mathrm{T}}^{e}}$, namely $[3,4[\mathrm{GeV} / c$, $[4,6[\mathrm{GeV} / c,[6,9[\mathrm{GeV} / c$ and $[9,18[\mathrm{GeV} / c$. The equation 6.1] summarizes the calculation for obtaining the heavy-flavour decay electron-jet (HFe-jet) spectra $\frac{d^{2} N^{H F e}}{d \eta d p_{\mathrm{T}, \mathrm{chjet}}}$.

$$
{\frac{d^{2} N\left(I_{p_{\mathrm{T}}^{e}}\right)}{d \eta d p_{\mathrm{T}, \mathrm{chjet}}}}^{H F e}=\frac{1}{\epsilon_{H F e I D}\left(I_{p_{\mathrm{T}}^{e}}\right)}\left\{J^{\text {incl. }}\left(I_{p_{\mathrm{T}}^{e}}\right)-J^{\text {had. }}\left(I_{p_{\mathrm{T}}^{e}}\right)-\frac{J^{\text {conv. }}\left(I_{p_{\mathrm{T}}^{e}}\right)}{\epsilon_{i n v}\left(I_{p_{\mathrm{T}}^{e}}\right)}\right\}
$$

One must select the inclusive electron-jet pairs $J^{\text {incl. }}$ and subtract from them the hadronic contamination $J^{\text {had. }}$ and the non-HF electron contamination $J^{c o n v}$. The efficiency of the invariant mass method $\epsilon_{i n v}$ and efficiency of the heavy-flavour identification method 
$\epsilon_{H F e I D}$ must be taken into account according to the equation. $\frac{d^{2} N}{d \eta d p_{\mathrm{T}, \text { chjet }}}$ is the number of e-jet pairs per unity of jet pseudo rapidity $\eta$ and per $p_{\text {T,chjet }}$. The contributions are calculated for each $I_{p_{\mathrm{T}, \mathrm{chjet}}}$ and summed (equation 6.2).

$$
\frac{d^{2} N^{H F e}}{d \eta d p_{\mathrm{T}, \text { chjet }}}=\sum_{I_{p_{\mathrm{T}}^{e}}} \frac{d^{2} N\left(I_{p_{\mathrm{T}}^{e}}\right)^{H F e}}{d \eta d p_{\mathrm{T}, \text { chjet }}}
$$

The inclusive electron contribution $J^{\text {incl. }}$ will be discussed in section 6.3. The hadronic contribution $J^{\text {had. }}$ calculation (equation 6.4) will be discussed in section 6.4. The conversion contribution $J^{c o n v}$. calculation and its method efficiency $\epsilon_{i n v}$. are discussed in section 6.5. Section 6.6 describes the heavy-flavour decay electron identification efficiency $\epsilon_{H F e I D}$.

The e-jet pairs were also divided in different $\Delta \varphi=\varphi_{\text {jet }}-\varphi_{e}$ regions, as shown in figure 6.1. $\Delta \varphi \in[0, \pi / 10[$ (near side), $\Delta \varphi \in[\pi / 10, \pi / 2[$ (middle) and $\Delta \varphi \in[\pi / 2, \pi[$ (away side). For each of them, the analysis procedure is exactly the same, being the only difference the $\Delta \varphi$ projection.

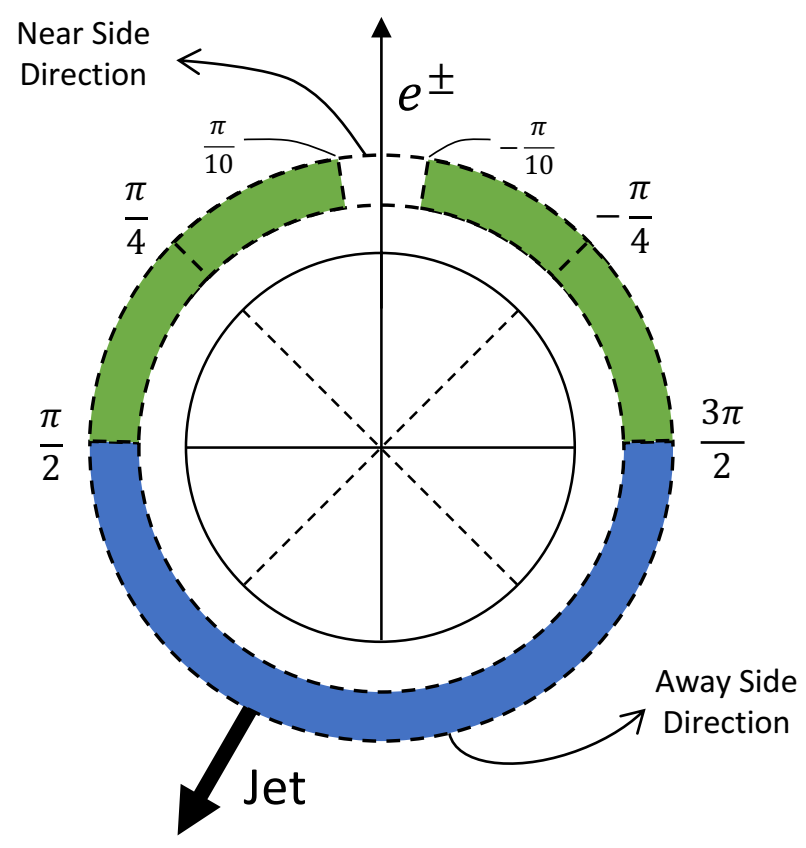

Figure 6.1: Electron-jet angles scheme. The blue region represents the away side region; the white one, the near side; the green one is the middle region. Note that the distances can be reduced to a $[0, \pi]$ range without losing information.

\subsection{Inclusive Electron-Jet Selection}

The first selection of the electron-jet pairs consists in constraining the $N_{\sigma T P C}^{e}$ to the electron region $[-1.5,3.0]$, where other species contamination is not dominant. The second step consists in selecting tracks with $E / p$ signal in the region $[0.8,1.3]$, where the electron purity is higher. Both selections are shown in figure 6.2 . 

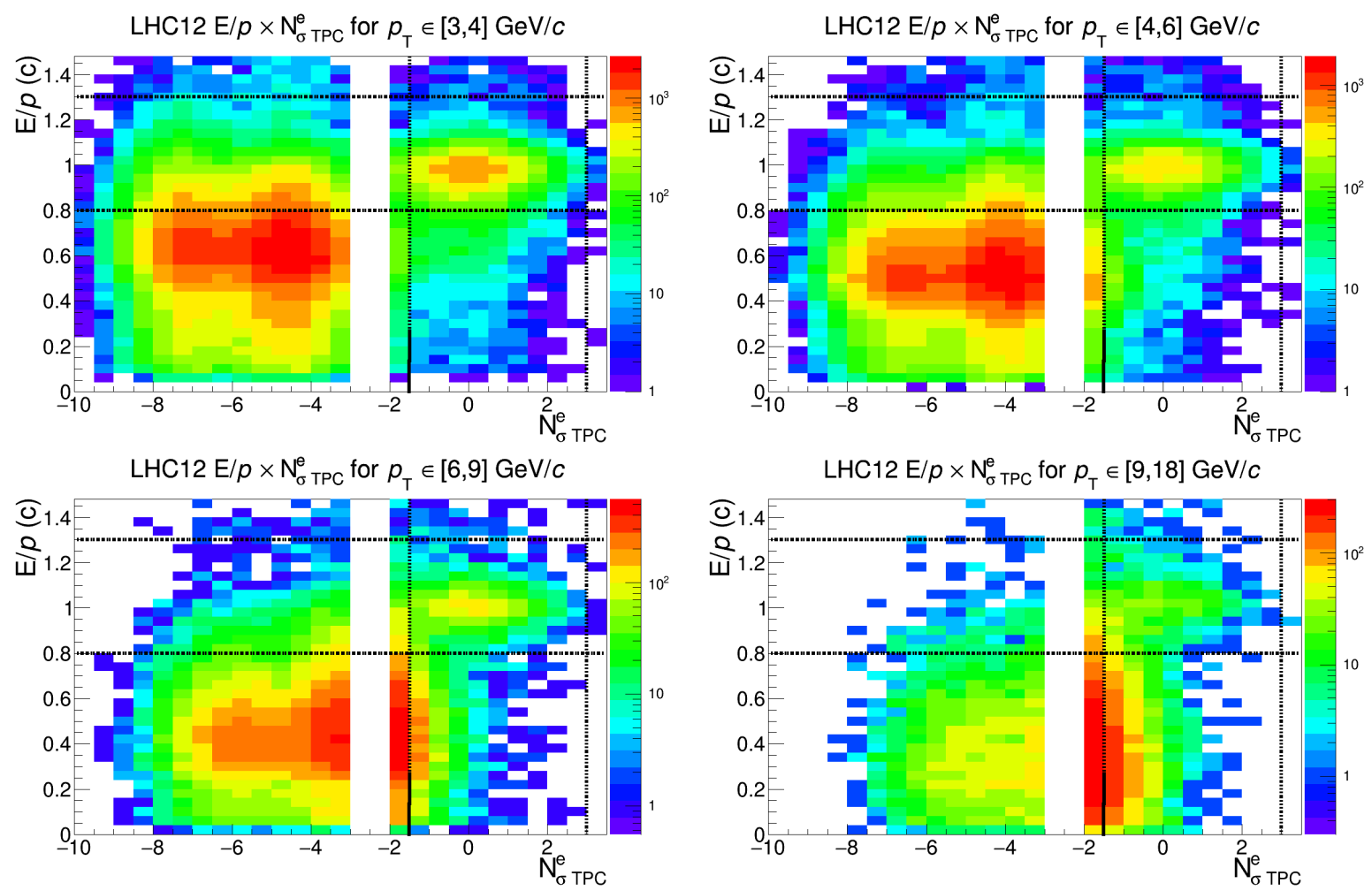

Figure 6.2: Electron-jet inclusive selection (TPC + EMCal) (pp). The black, dashed lines show the selection region. Each panel contains a different $p_{\mathrm{T}}^{e}$ range: top left $[3,4] \mathrm{GeV} / c$, top right $[4,6] \mathrm{GeV} / c$, bottom left $[6,9] \mathrm{GeV} / c$, bottom right $[9,18] \mathrm{GeV} / c$. The empty region delimitates two different statistics regions (see appendix A.3): the lefthand side has $80 \%$ of its total statistics discarded randomly, while the righthand side does not. The data are from pp collisions, taken in 2012 by ALICE.

After this inclusive electron selection, one gets the heavy-flavour electron candidates-

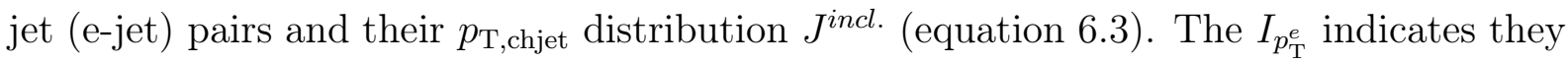
are separated in different $p_{\mathrm{T}}^{e}$ intervals.

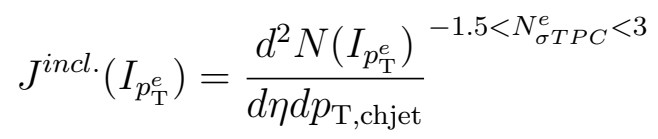

\subsection{Hadronic Contamination}

The inclusive set of e-jet pairs still includes some non-electron particles, mainly hadrons that are misidentified, that must be estimated and subtracted from the inclusive set. The estimate is done by comparing the $E / p$ distribution of hadrons and inclusive electrons.

In order to subtract the hadronic contamination from the e-jet spectra, hadron-jet spectra are generated applying the same set of cuts as previously done, except for the $N_{\sigma T P C}^{e}$ cut that is applied in the hadronic region $[-10,-3]$ resulting in a similar distribution (equation 6.4): 


$$
J^{\text {had. }}\left(I_{p_{\mathrm{T}}^{e}}\right)=\frac{f_{h}\left(I_{p_{\mathrm{T}}^{e}}\right)}{A_{\text {norm }}\left(I_{p_{\mathrm{T}}^{e}}\right)} \frac{d^{2} N\left(I_{p_{\mathrm{T}}^{e}}\right)^{-10<N_{\sigma T P C}^{e}<-3}}{d \eta d p_{\mathrm{T}, \mathrm{chjet}}}
$$

The hadronic $E / p$ distribution is rescaled (by the constant $A_{\text {norm. }}$ ) in order to have its integral, in the range $E / p \in[0.24,0.74]$, normalized to the electron distribution, in the same range, since no electrons should be found in this region of the $E / p$ distribution. Once the two distributions are normalized, one can calculate the relative area of the hadronic $E / p$ distribution to the electron one, in the region $E / p \in[0.8,1.3]$, to obtain the value of $f_{h}$.

The values $A_{\text {norm. }}$ and $f_{h}$ are calculated for each $p_{\mathrm{T}}^{e}$ bin (as shown in figures 6.3 6.4 and 6.5. The hadron-jet $p_{T, c h j e t}$ distribution $\frac{d^{2} N\left(I_{p_{T}^{e}}\right)}{d \eta d p_{T, \text { chjet }}}$ is normalized and subtracted from the inclusive $J^{\text {incl. }}$ distribution.

LHC12 E/p for $p_{\mathrm{T}} \in[3.0,4.0] \mathrm{GeV} / c$ and $\Delta \varphi \in[\pi / 2, \pi]$

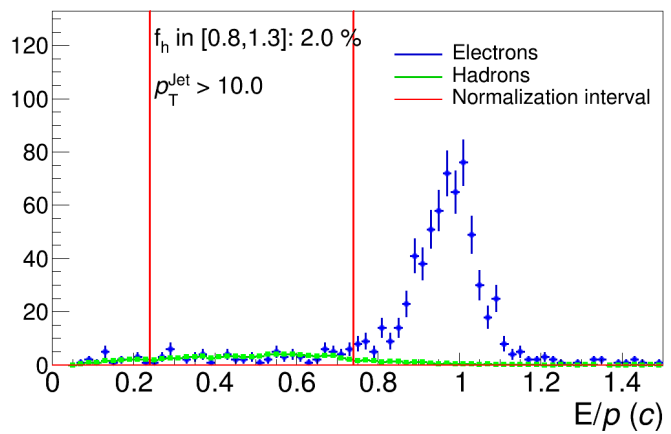

$\mathrm{LHC} 12 \mathrm{E} / p$ for $p_{\mathrm{T}} \in[6.0,9.0] \mathrm{GeV} / c$ and $\Delta \varphi \in[\pi / 2, \pi]$

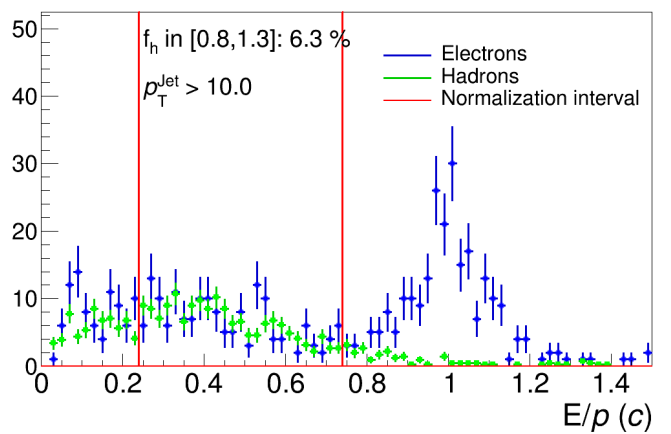

LHC12 E/p for $p_{T} \in[4.0,6.0] \mathrm{GeV} / c$ and $\Delta \varphi \in[\pi / 2, \pi]$

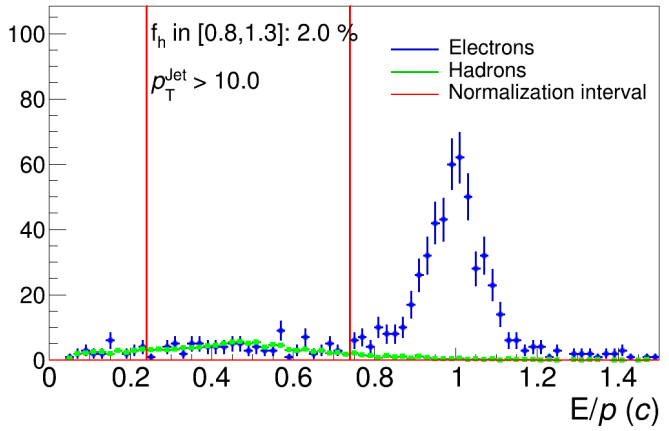

LHC12 $\mathrm{E} / p$ for $p_{\mathrm{T}} \in[9.0,18.0] \mathrm{GeV} / c$ and $\Delta \varphi \in[\pi / 2, \pi]$

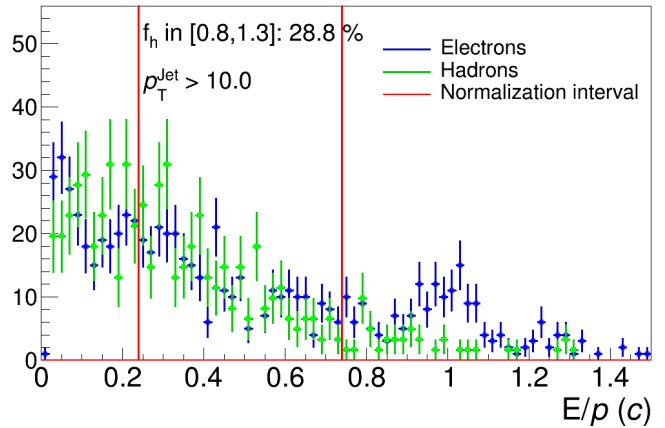

Figure 6.3: Away side Electron-jet $E / p$ hadronic contamination: In blue, the inclusive electron distribution; in green, the hadronic contribution. The red bars show the normalization interval used in the $A_{\text {norm. }}$. calculation. The data are from pp collisions, taken in 2012 by ALICE. 
LHC12 E/p for $p_{\mathrm{T}} \in[3.0,4.0] \mathrm{GeV} / c$ and $\Delta \varphi \in[0, \pi / 10]$

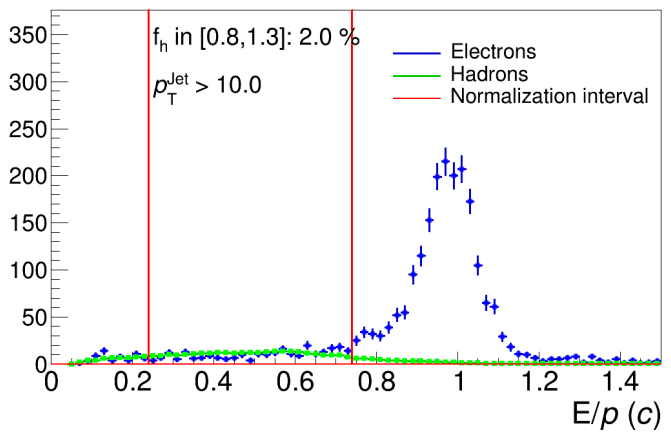

LHC12 $\mathrm{E} / p$ for $p_{\mathrm{T}} \in[6.0,9.0] \mathrm{GeV} / c$ and $\Delta \varphi \in[0, \pi / 10]$

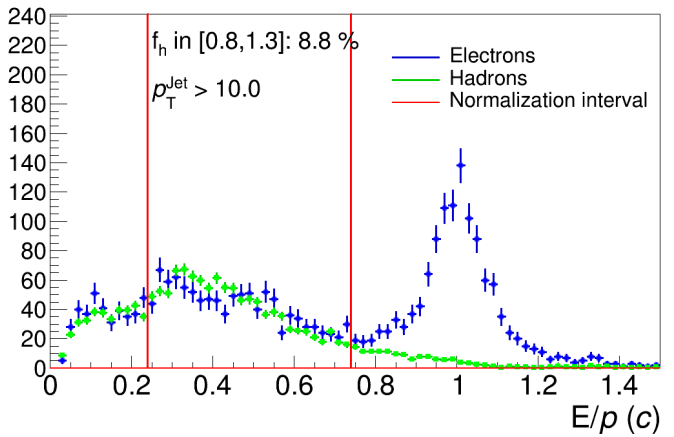

LHC12 $\mathrm{E} / p$ for $p_{\mathrm{T}} \in[4.0,6.0] \mathrm{GeV} / c$ and $\Delta \varphi \in[0, \pi / 10]$

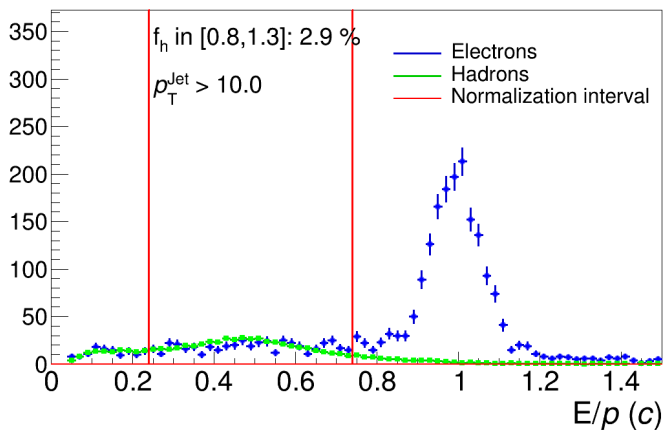

LHC12 E/p for $p_{\mathrm{T}} \in[9.0,18.0] \mathrm{GeV} / c$ and $\Delta \varphi \in[0, \pi / 10]$

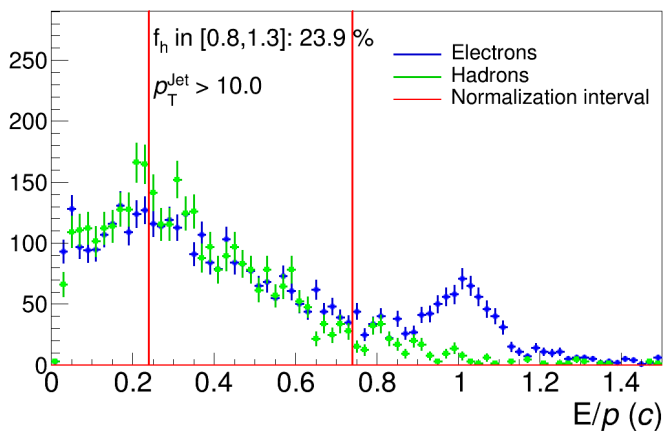

Figure 6.4: Near side Electron-jet $E / p$ hadronic contamination: In blue, the inclusive electron distribution; in green, the hadronic contribution. The red bars show the normalization interval used in the $A_{\text {norm. }}$ calculation. The data are from pp collisions, taken in 2012 by ALICE.

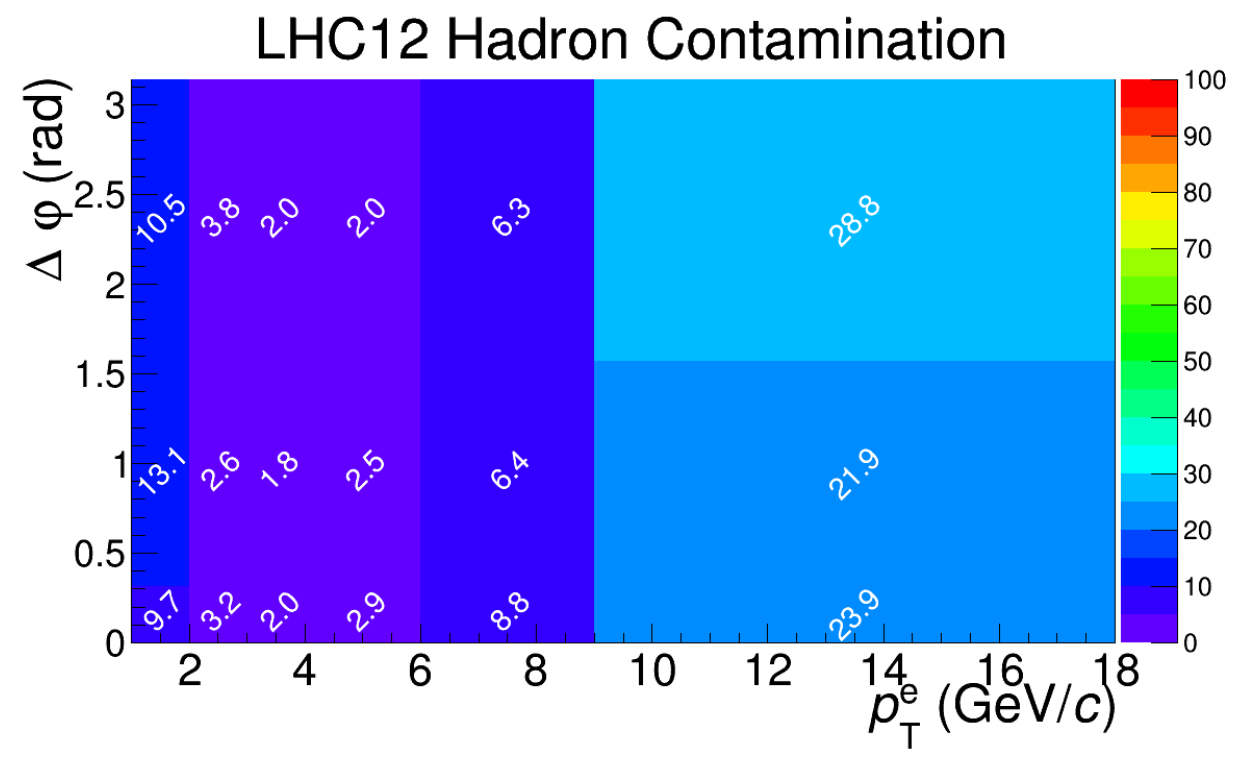

Figure 6.5: Hadronic contamination percentages $f_{h}$ for each $p_{\mathrm{T}}^{e} \times \Delta \varphi$ bin. The data are from pp collisions, taken in 2012 by ALICE. 


\subsection{Non-HF Electron Contamination}

After the subtraction of the hadronic contribution, the non heavy-flavour electron contribution $J^{c o n v}$, that is mainly produced via photonic and pionic contributions, remains in the resulting sample. As explained in section 5.5, this contribution can be estimated using the invariant mass technique. Again, the estimation must be obtained for each $p_{\mathrm{T}}^{e}$ bin so the efficiencies can be taken into account.

Figure 6.6 shows the low invariant mass distribution, where a clear peak from nonHF decay electrons is visible. The $p_{T, \text { chjet }}$ projections for the mass cut $m_{e^{+} e^{-}}<0.135$ $\mathrm{GeV}$, namely $J_{L S}^{m<C u t}$ and $J_{U L S}^{m<C u t}$, are used in the calculation of the non-HF electron contribution $J^{\text {conv. }}$ (equation 6.5):

$$
J^{\text {conv. }}\left(I_{p_{\mathrm{T}}^{e}}\right)=\frac{J_{U L S}^{m<C u t}\left(I_{p_{\mathrm{T}}^{e}}\right)-J_{L S}^{m<C u t}\left(I_{p_{\mathrm{T}}^{e}}\right)}{\epsilon_{i n v}\left(I_{p_{\mathrm{T}}^{e}}\right)}
$$
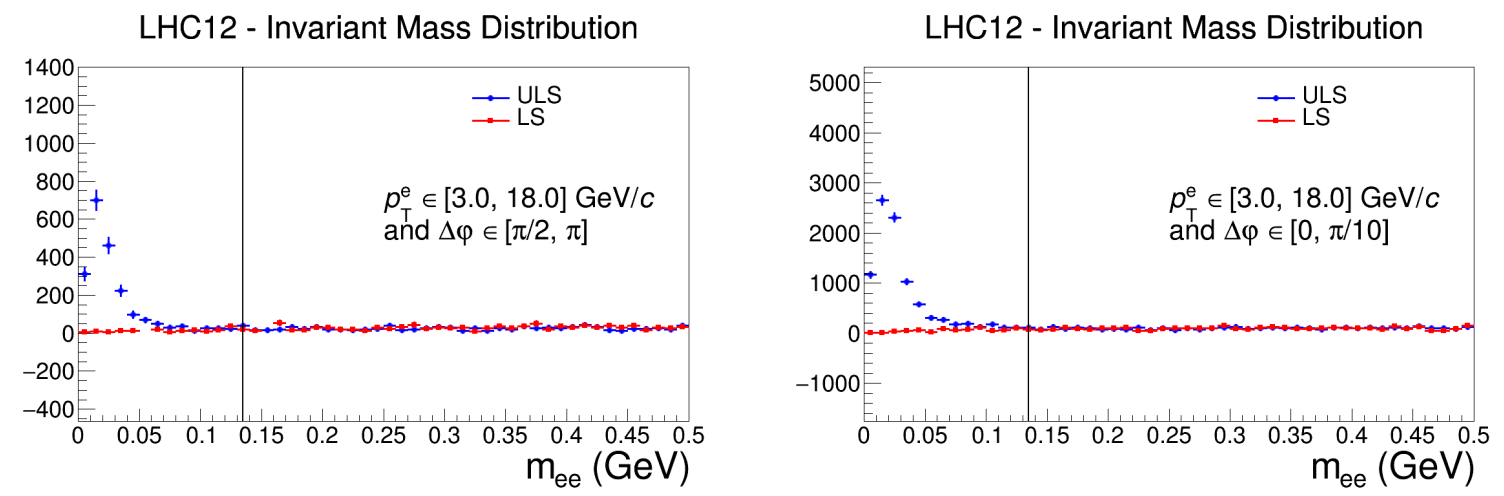

Figure 6.6: Invariant mass distribution for like-sign $e^{+} e^{-}$pairs (in red) and for unlike-sign pairs (in blue). The black bar shows the mass cut $0.135 \mathrm{GeV}$ : only the region to its left is used in the non-HF electron contribution. The left panel shows the away side and the right panel shows the near side. The data are from ALICE pp collisions.

The efficiency of the invariant mass method $\epsilon_{\text {inv }}$ was calculated from a Monte Carlo simulation (LHC1512b2 period). The exact same selections and cuts were applied to the generated events and particles in order to obtain the non-HFe $p_{\mathrm{T}}^{e}$ distribution $d^{2} N / d \eta d p_{\mathrm{T}}^{e}$ conv. from the invariant mass method, like in data. In addition, the generator information about

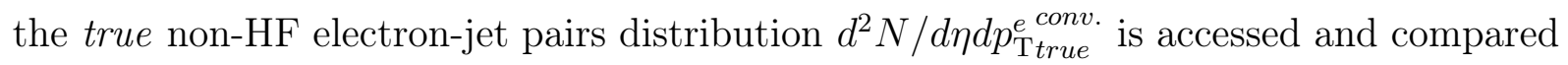
to the latter. The efficiency $\epsilon_{i n v}$. (equation 6.6) is taken as their ratio, as shown in figure 6.7 .

$$
\epsilon_{\text {inv. }}=\frac{{\frac{d^{2} N}{d \eta d p_{\mathrm{T}}^{e}}}^{\text {conv. }}}{\frac{d^{2} N}{d \eta d p_{\mathrm{T}}^{e}} \text { true }}
$$




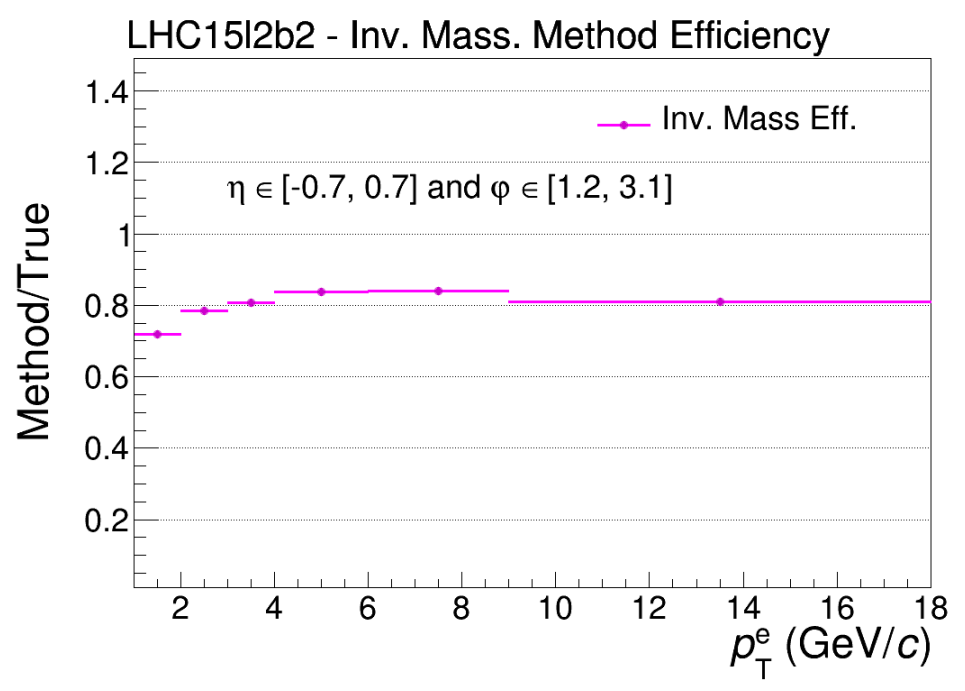

Figure 6.7: Invariant mass method efficiency. The data are from ALICE pp simulations.

\subsection{Electron Identification Efficiency}

Although the electron sample is highly pure, not all the heavy-flavour decay electrons are detected. This must be corrected by a detection efficiency, that can be calculated using a Monte Carlo simulation.

In the simulation (LHC16a1c period), one can compare the number of generated heavyflavour decay electrons $d N / d p_{\mathrm{T}}^{\text {etrue } H F}$ to the number of the electrons that passed all the selection and identification steps, $d N / d p_{\mathrm{T}}^{e^{I D} H F}$. Their ratio (equation 6.7)

$$
\epsilon_{H F e I D}=\frac{d^{2} N / d \eta d p_{\mathrm{T}}^{e^{I D} H F}}{d^{2} N / d \eta d p_{\mathrm{T}}^{e^{\text {true } H F}}}
$$

is the efficiency $\epsilon_{H F e I D}$ shown in figure 6.8, that can be applied to the detected e-jet pairs in the real data analysis. The analysis in simulation was performed exactly as in data, but including also the generator information.

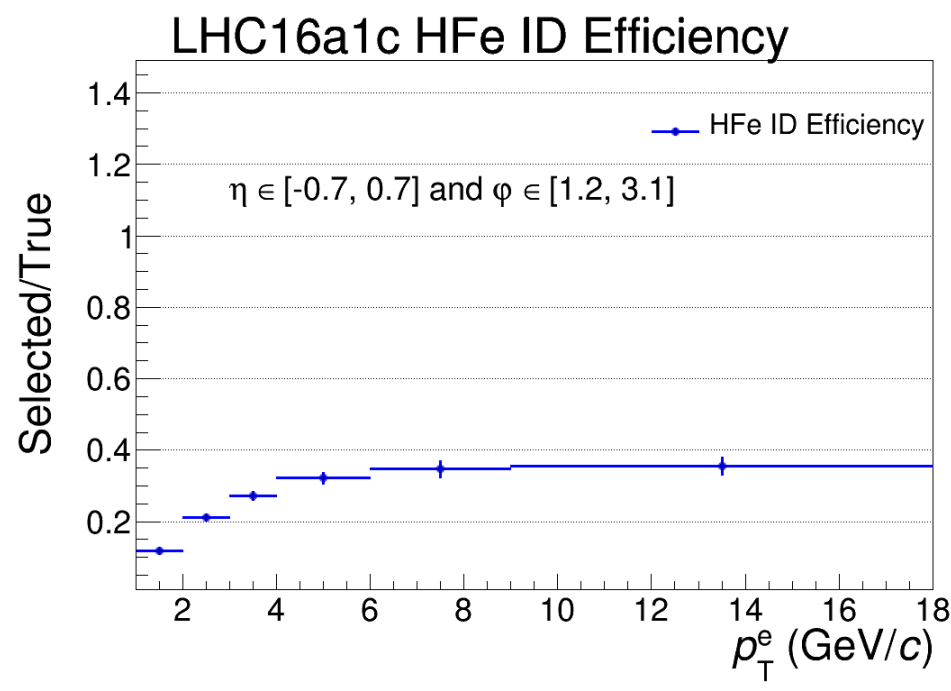

Figure 6.8: Heavy-flavour decay electron identification efficiency. The data is from an ALICE simulation (LHC16a1c period) for pp collisions. 


\subsection{HF Electron-Jet $\Delta \varphi$}

Figure 6.9 shows the inclusive, hadronic and non-HF electron $\Delta \varphi$ distributions, for the $p_{\mathrm{T}}^{e} \in[3,4] \mathrm{GeV}$ interval. This is an alternative way to analyze the data besides the $p_{\mathrm{T}, \mathrm{chjet}}$ spectra. Equation 6.8 shows the calculation of this observable (similar to 6.1):

$$
\begin{aligned}
\frac{d^{2} N\left(I_{p_{\mathrm{T}}^{e}}\right)^{H F e}}{d \eta d \Delta \varphi}=\frac{1}{\epsilon_{H F e I D}\left(I_{p_{\mathrm{T}}^{e}}\right)} & \left\{\frac{d^{2} N\left(I_{p_{\mathrm{T}}^{e}}\right)^{-1.5<N_{\sigma T P C}^{e}<3}}{d \eta d \Delta \varphi}\right. \\
& -\frac{f_{h}\left(I_{p_{\mathrm{T}}^{e}}\right)}{A_{n o r m}\left(I_{p_{\mathrm{T}}^{e}}\right)} \frac{d^{2} N\left(I_{p_{\mathrm{T}}^{e}}\right)^{-10<N_{\sigma T P C}^{e}<-3}}{d \eta d \Delta \varphi} \\
& \left.-\frac{\mathcal{J}_{U L S}^{m<C u t}\left(I_{p_{\mathrm{T}}^{e}}\right)-\mathcal{J}_{L S}^{m<C u t}\left(I_{p_{\mathrm{T}}^{e}}\right)}{\epsilon_{i n v}\left(I_{p_{\mathrm{T}}^{e}}\right)}\right\}
\end{aligned}
$$

where the first summand is the inclusive e-jet contribution; the second, is the hadronic contribution; the $\mathcal{J}$ are the projection of the $\Delta \varphi$ distribution for like-sign (LS) and unlikesign (ULS) electron-electron pairs, for the invariant mass $m_{e e}<0.135 \mathrm{GeV} ; \epsilon_{H F e I D}$ is the heavy-flavour decay electron identification efficiency and $\epsilon_{i n v}$ is the invariant mass method efficiency. Since this calculation is performed for the different electron $p_{\mathrm{T}}^{e}$ intervals $I_{p_{\mathrm{T}}^{e}}$, the final formula is the summation 6.9 for all of them.

$$
\frac{d^{2} N^{H F e}}{d \eta d \Delta \varphi}=\sum_{I_{p_{\mathrm{T}}^{e}}} \frac{d^{2} N\left(I_{p_{\mathrm{T}}^{e}}\right)^{H F e}}{d \eta d \Delta \varphi}
$$

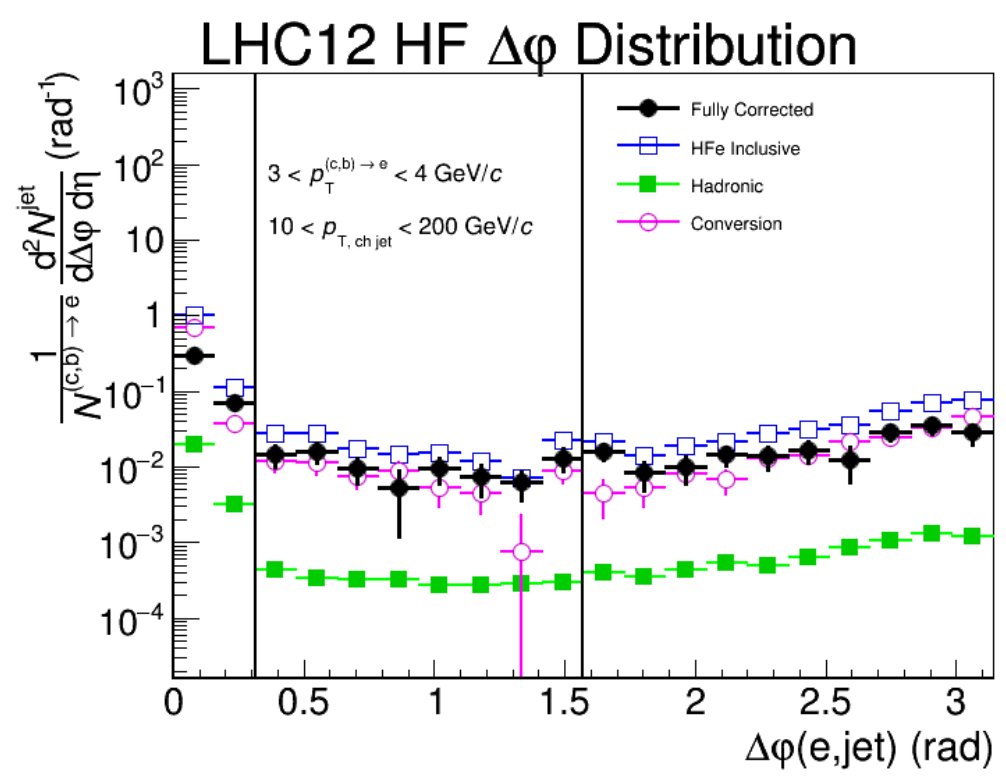

Figure 6.9: HFe-jet $\Delta \varphi$ normalized distributions: in black, the corrected one; in blue, the inclusive; the hadronic contribution is in green; and the non-HF electron contribution is in magenta. Vertical lines are the divisions between away side, near side and middle. The data are from 2012 pp collisions. 


\subsection{Unfolding}

The unfolding procedure was performed using the RooUnfold [61] libraries, with the Iterative Bayesian method. This framework has as input the measured spectrum $\frac{d^{2} N^{H F e}}{d \eta d p_{\text {T,chjet }}}$, after the FONLL energy scaling (to be explained in section 8.1 and also in appendix A.8, and the response matrix.

The response matrix corresponds to the detector response matrix for jet measurements (section 3.4). The unfolded spectrum is the output after 3 iterations (parameter $K$ was set to 3). Appendix A.7 discusses a study on the uncertainties that are related to the unfolding procedure.

\section{Detector Response}

Using a Monte Carlo simulation information (LHC16a1c period from ALICE), one can associate two sets of jets:

- the generator level jets, which were reconstructed from the set of particles directly obtained from the generator, without any detector effects;

- the reconstructed level jets, which were reconstructed from the set of tracks that have detector effects accounted;

The association between generated and reconstructed jets was performed using the closest distance between these objects, considering that $\Delta R_{\text {jets }}=\sqrt{\Delta \varphi_{\text {jets }}^{2}+\Delta \eta_{\text {jets }}^{2}}<$ 0.2. The jet pairs were also associated to electrons candidates (with the looser cuts $N_{\sigma T P C}^{e} \in[-3.5,3.5]$ and $\left.E / p \in[0.7,1.3]\right)$ in the event so they could be divided in the three angular regions (away side, near side and middle) in order to account for possible bias in the jet selection. Figure 6.10 shows the away and near side response matrices for pp collisions.
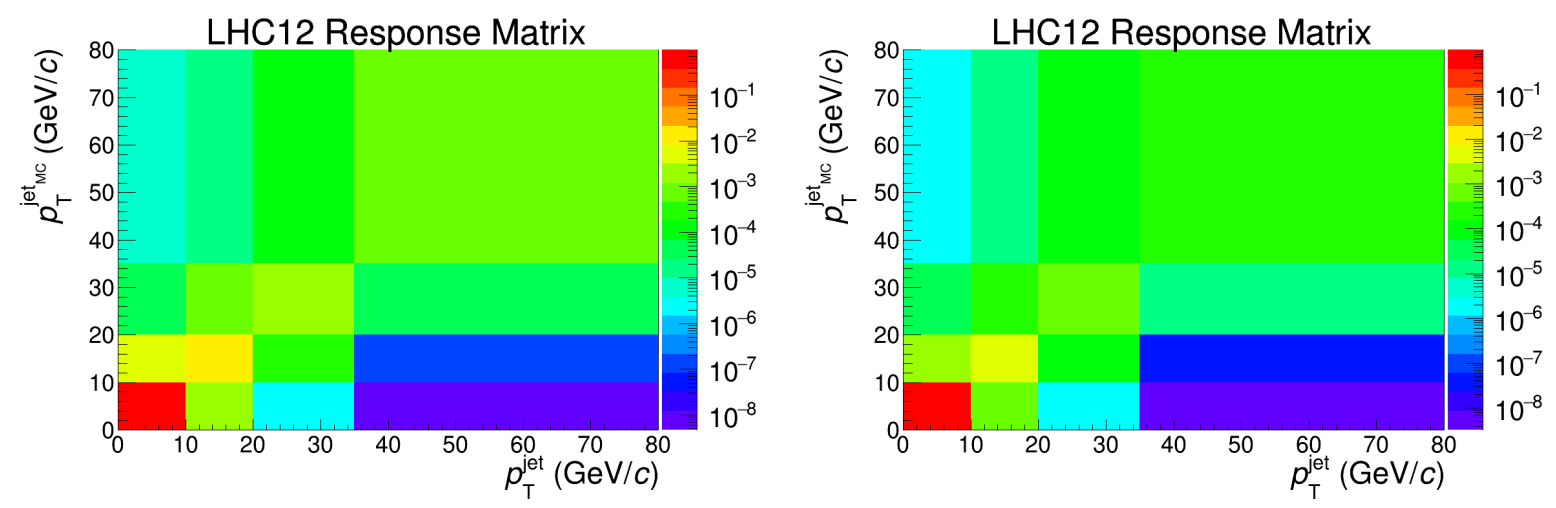

Figure 6.10: Away side response matrix is in the left-hand side. Near side response matrix is in the right-hand side. The data is from an ALICE simulation for pp collisions. 
The jets that were not paired were also included in the matrix, in a separated bin. Therefore, the projection into the reconstructed level axis can include or not these jets, making it possible to calculate the efficiency of finding a generator level jet, i.e. the ratio between the number of found jets and the number of total jets. RooUnfold objects deals with these information automatically during the unfolding procedure.

\subsection{HF Electron-Jet Spectrum}

All the calculated contributions - namely inclusive, hadronic and non-HF electrons are already corrected by the efficiencies but they still need to be normalized.

\section{Normalization}

In order to obtain an observable that is independent of the number of events that were analysed, it has to be normalized. The normalization of the $p_{\mathrm{T} \text {,chjet }}$ and the $\Delta \varphi$ distribution was taken as the number of heavy-flavour decay electrons $N_{H F e}$ used in the analysis. It is calculated according to equation 6.10 .

$$
N_{H F e}=\frac{1}{\epsilon_{H F e I D}}\left\{N^{-1.5<N_{\sigma T P C}<3.0}\left(1-f_{h}^{\text {tracks }}\right)-\frac{N_{U L S}-N_{L S}}{\epsilon_{\text {inv. }}}\right\}
$$

The main difference to the equations 6.1 and 6.10 is that a single track is used instead of a e-jet pair. $N^{-1.5<N_{\sigma T P C}<3.0}$ is the number of tracks in this $N_{\sigma T P C}^{e}$ region. The hadronic contamination fraction $f_{h}^{\text {tracks }}$ is calculated using the $E / p$ distribution (figure 6.11). The invariant mass method is used to calculate the non-HFe contribution $N_{U L S}-N_{L S}$ : the electron-electron, positron-positron and electron-positron pairs are used. The normalization was also rescaled according to FONLL (to be discussed in section 8.1).

Figure 6.12 shows the three contributions and the corrected spectrum. The normalization $N_{H F e}$ is the integral of the corrected distribution.

The final, corrected, scaled and unfolded jet $p_{\mathrm{T}, \text { chjet }}$ spectrum is shown, for the away and near side regions, in figures 6.13 and 6.14 . 

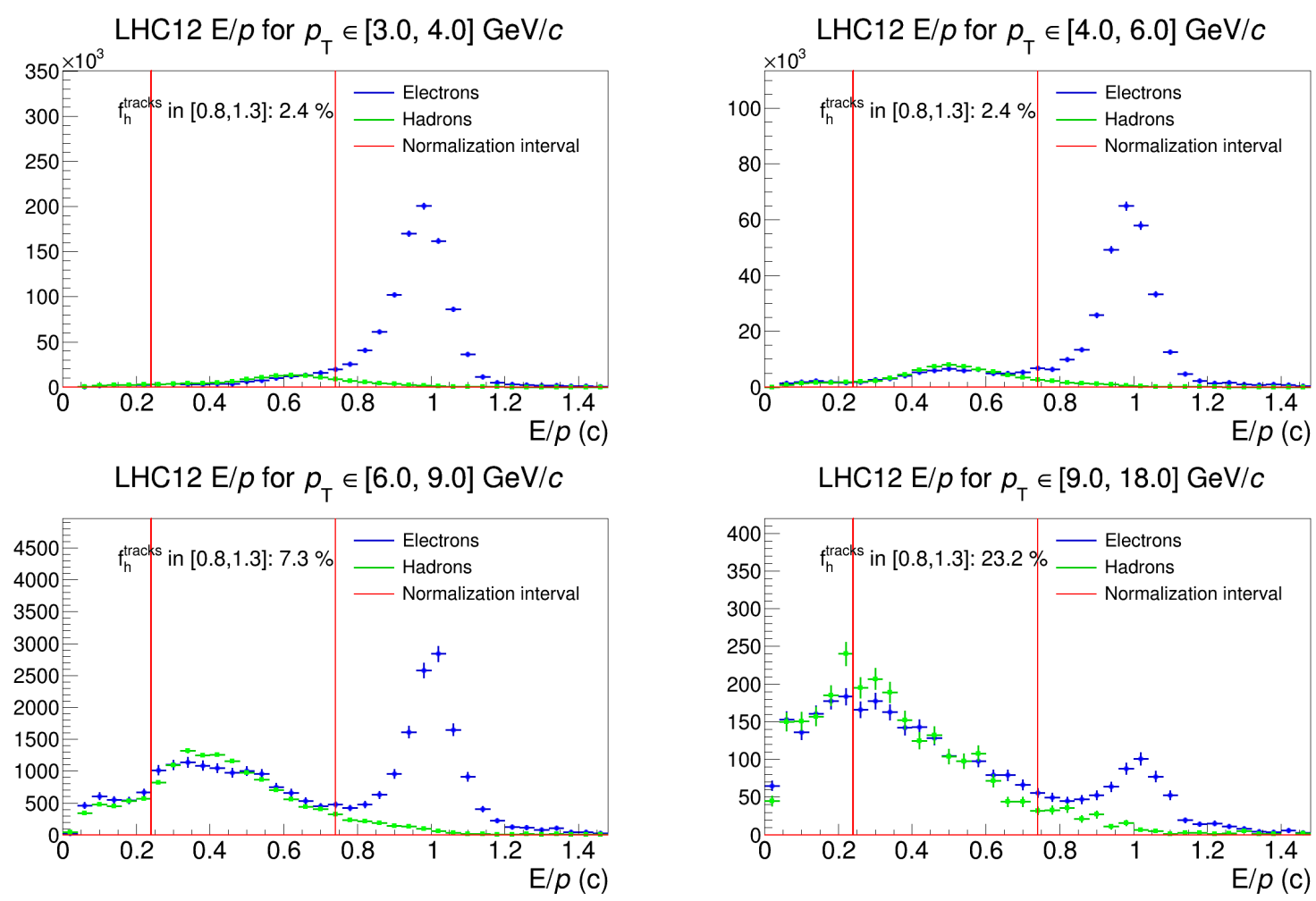

LHC12 E/p for $p_{\mathrm{T}} \in[9.0,18.0] \mathrm{GeV} / c$

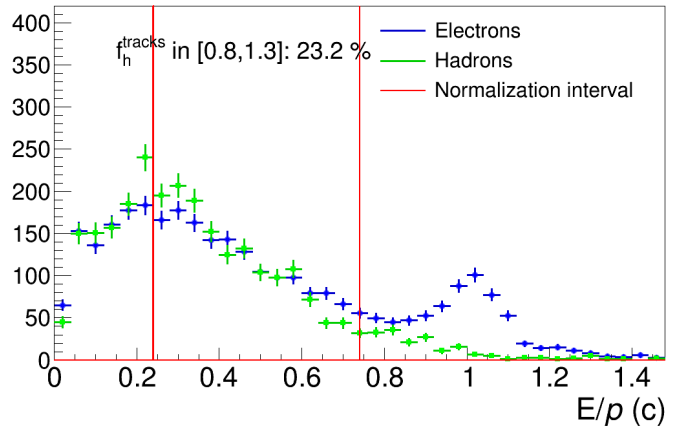

Figure 6.11: Hadronic contamination fractions for tracks only (not e-jet pairs). The blue points are the inclusive electron distribution, the green points are the estimated hadronic distribution and the bars highlight the normalization interval. Data from 2012 pp collisions.

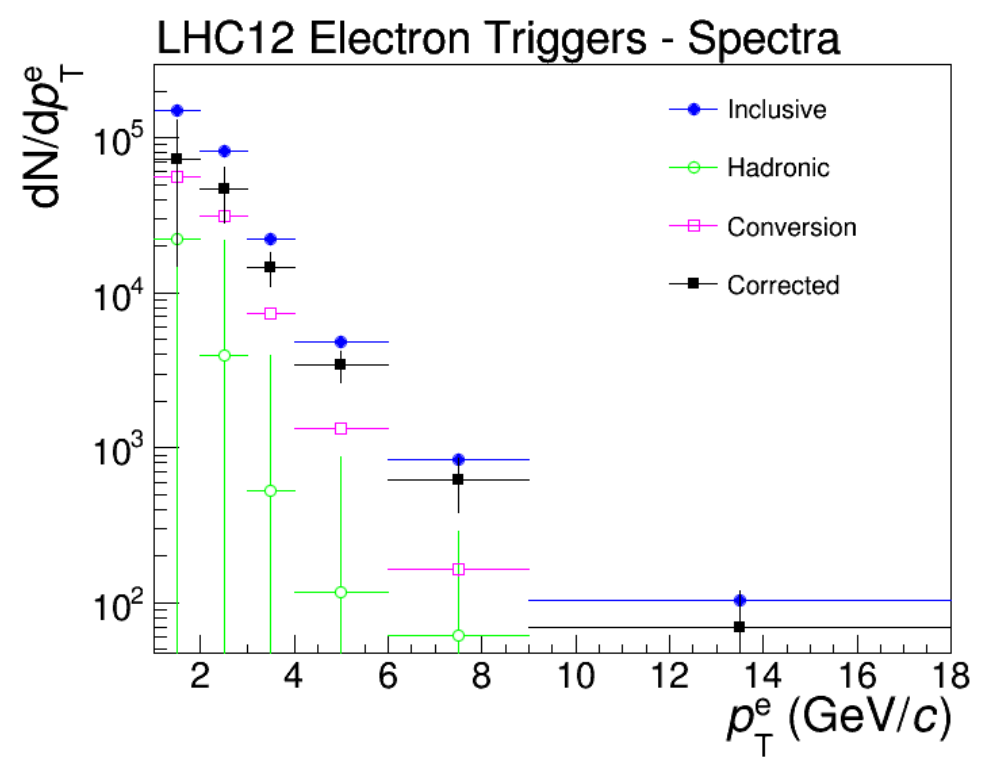

Figure 6.12: Heavyflavour electron $p_{\mathrm{T}}^{e}$ spectrum. The three contributions (not normalized) are shown separately: inclusive is in blue; hadronic is in green; non-HF electron is in magenta. The corrected spectra is in black and its integral, in the proper range, is the normalization for the e-jet observables. Data from pp collisions. 

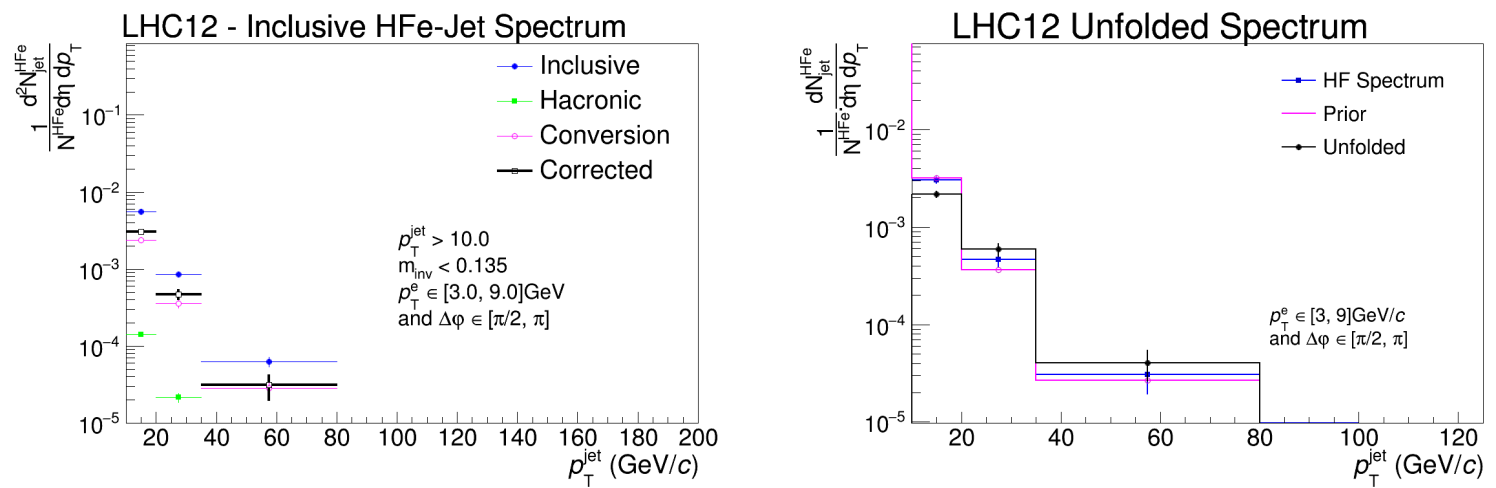

Figure 6.13: Away side: In the left panel, the contributions for the e-jet distribution are shown, namely inclusive (blue), hadronic (green) and non-HF electron (magenta). The corrected one is in black. In the right panel, the unfolded spectrum is shown. The prior used in the unfolding procedure is in magenta. Data from pp collisions.
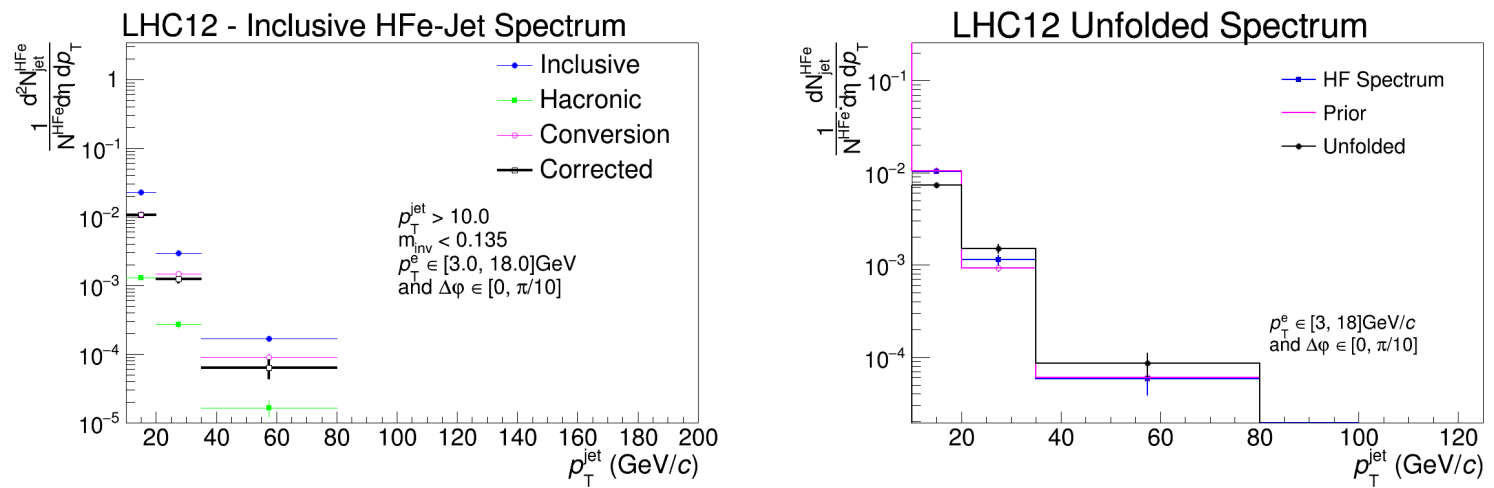

Figure 6.14: Near side: In the left panel, the contributions for the e-jet distribution are shown, namely inclusive (blue), hadronic (green) and conversion (magenta). The corrected one is in black. In the right panel, the unfolded spectrum is shown. The prior used in the unfolding procedure is in magenta. Data from pp collisions. 


\subsection{Systematic Uncertainties}

The systematic uncertainty evaluation was done by comparing the normalized resulting spectra $\frac{1}{N_{H F e}} \frac{d^{2} N}{d \eta d p_{\text {T,chjet }}}{ }^{H F e}$ from different variations of cuts and selections. Table 6.1 shows all the variations: each was changed while all the others were kept as the standard cut. The difference between the standard and the variation spectra $\Delta_{\text {Barlow }}$ thus given by equation 6.11.

$$
\Delta_{\text {Barlow }}=\frac{1}{N_{H F e}}{\frac{d^{2} N}{d \eta d p_{\mathrm{T}, \text { chjet }}}}^{H F e}-{\frac{1}{N_{H F e}^{v a r .}}}_{\frac{d^{2} N}{d \eta d p_{\mathrm{T}, \text { chjet }}}}^{H F e, \text { var }}
$$

If the difference $\Delta_{\text {Barlow }}$ between the standard and the varied spectra respected the Barlow's criterion [62] (equation 6.12, where $\sigma$ is the statistical uncertainty), $\Delta_{\text {Barlow was }}$ accounted in the systematic uncertainty calculation.

$$
\frac{\Delta_{\text {Barlow }}}{\sqrt{\sigma_{s}^{2}-\sigma_{v a r}^{2}}}>1.0
$$

For each group of variations, i.e. each line in the table, the square root of the mean square $\mathrm{RMS}_{\text {group }}$ was calculated. The final systematic uncertainty was chosen as the square sum of the RMS for each group (equation 6.13).

$$
\sigma_{\text {sys }}=\sqrt{\sum_{\text {group }} \mathrm{RMS}_{\text {group }}^{2}}
$$

This was done for each bin of the distribution. Figures 6.15 and 6.16 show the final spectra with the systematic uncertainties for the two $\Delta \varphi$ regions.

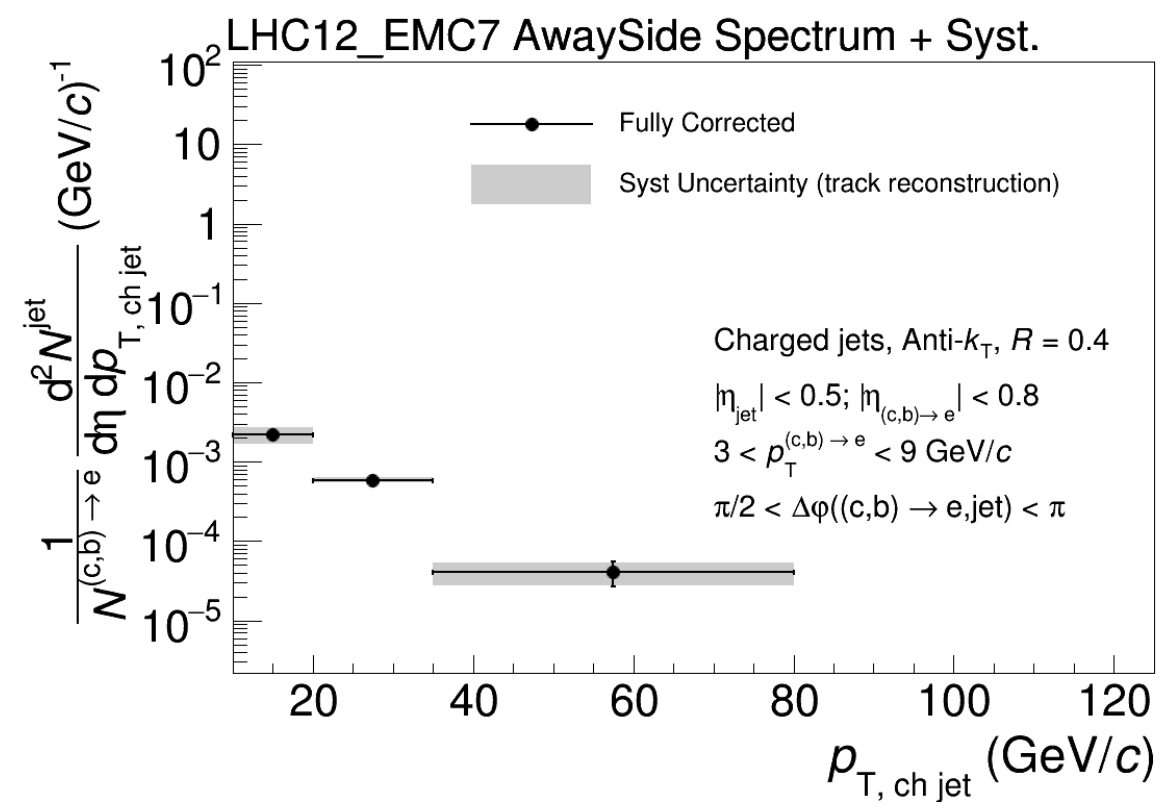

Figure 6.15: Away side: HFe-jet spectrum with systematic uncertainties. 


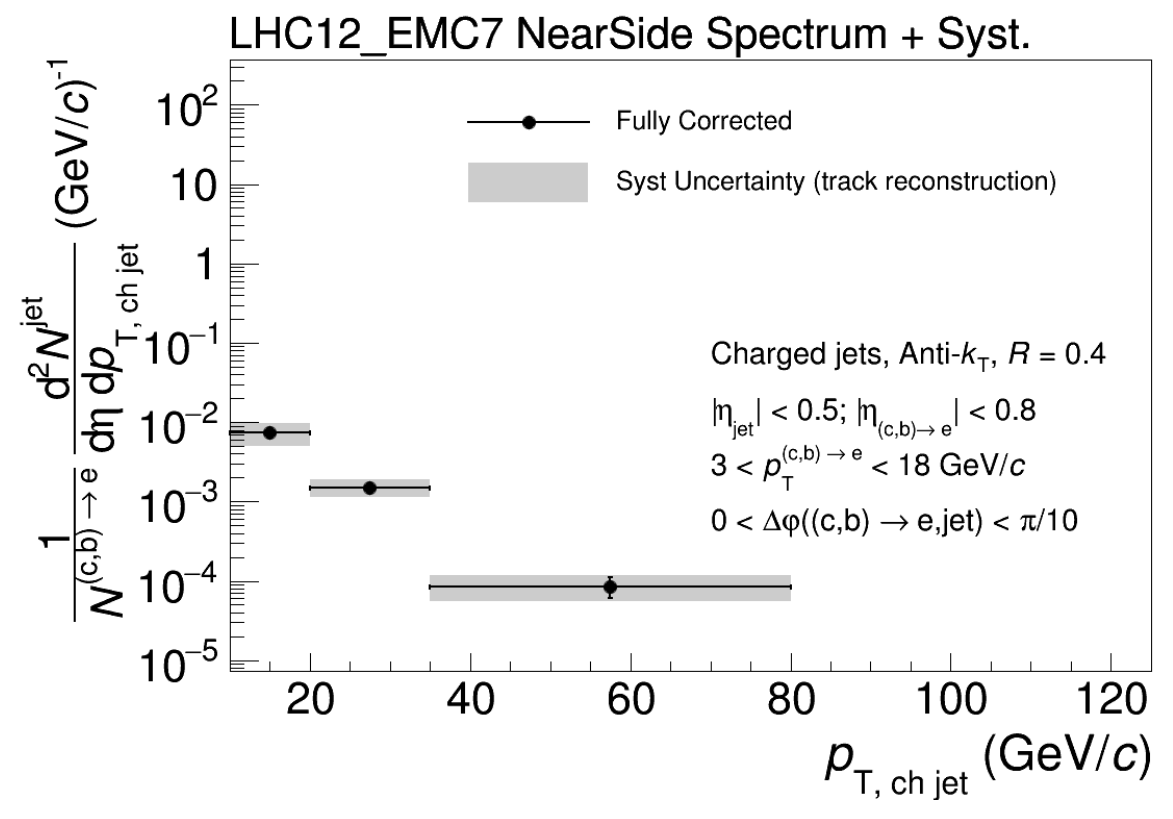

Figure 6.16: Near side: HFe-jet spectrum with systematic uncertainties. 
Table 6.1: Variation of cuts and selections for systematic uncertainty calculation in pp.

\begin{tabular}{|c|c|c|c|c|}
\hline Variable & Variation(s) & Standard & $\begin{array}{l}\text { Syst. Uncert. } \\
\text { Away side }\end{array}$ & $\begin{array}{l}\text { Syst. Uncert. } \\
\text { Near side }\end{array}$ \\
\hline Minimum TPC Clusters & 90,110 & 80 & & \\
\hline Min. TPC Clusters (PID) & 90,110 & 80 & $0 \%$ & \\
\hline$--\overline{\text { Min. }} \overline{\text { Hits }} \overline{\text { in }} \overline{\text { ITS }}$ & $-3,4$ & $-\overline{2}$ & -91 & $<\overline{9} \%$ \\
\hline $\begin{array}{l}-\cdots \overline{M i n i m u m} \\
N_{\sigma T P C}^{\bar{e}}\end{array}$ & $-1.0,-0.5,0.0,0 . \overline{2} 5,0.5$ & -1.5 & $24 \%$ & $10 \%$ \\
\hline Maximum $N_{\sigma}^{e} P_{-} C_{-}$ & $2.5,3.5$ & 3.0 & 2970 & $.10 / 0$ \\
\hline Minimum $\bar{E} / p$ & $0.76,0.78,0.82,0.84,0.86$ & 0.80 & $<2 \%$ & $<4 \%$ \\
\hline Maximum $E / p$ & $\ldots$ & -1.3 & & \\
\hline Maximum $M 20$ & $\begin{array}{r}0.2,0.4 \\
-\end{array}$ & 0.3 & $4 \%$ & $<14 \%$ \\
\hline Maximum $\left|\Delta \varphi_{\text {match }}\right|$ & 0.03 & 0.05 & $\approx 0 \%$ & $<$ \\
\hline Min. $\overline{\mathrm{T}} \overline{\mathrm{C}} \overline{\mathrm{C}}^{-} \mathrm{Clusters}$ (Partner) & 80 & 70 & $1 \%$ & $<$ \\
\hline Minimum $p_{\mathrm{T}}^{\bar{e}, \bar{p} a \bar{r} t \bar{n} e \bar{r}}$ & $1.0 \mathrm{G} \overline{\mathrm{GeV}} / c^{--}$ & $0.15 \mathrm{GeV} / \bar{c}$ & $<12 \%$ & $<29 \%$ \\
\hline Maximum $\bar{m}_{\text {inv. }}$ & $0 . \overline{100} \overline{\mathrm{G}} \overline{\mathrm{eV}}, 0.18 \overline{\mathrm{GeV}}$ & $0 . \overline{135} \overline{\mathrm{G}} \overline{\mathrm{eV}}$ & $-\overline{<} \overline{8} \overline{0}$ & $<\overline{5} \%$ \\
\hline Maximum $\left|N_{\sigma T P C}^{e}\right|{ }_{-}^{e}$ (Partner & $--\overline{2} . \overline{5}---$ & -5.0 & $\overline{1} \%$ & $\overline{1} \%$ \\
\hline 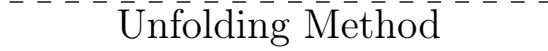 & $\overline{\mathrm{SVD}}$ & Iterative Bayesian & & \\
\hline Unfolding Parameter $K$ & $K+1$ & $K=3$ & $<20 \%$ & $<24 \%$ \\
\hline Unfolding Prior & Power Law & Response Y-Projection & & \\
\hline
\end{tabular}




\section{Analysis in Proton-Lead Data}

In this chapter, the $\mathrm{p}-\mathrm{Pb}$ analysis will be described in details. In section 7.1 , the event selection criteria are discussed. In section 7.3 , the electron identification and the selection of inclusive e-jet pairs are shown. Sections 7.4 and 7.5 describe the contamination estimation and their subtraction. Section 7.6 details the efficiency calculation for the electron selection. The unfolding technique procedure is described in 7.8 . The final observables are shown in section 7.9 and their systematic errors are shown in section 7.10 . Most of the procedures for $\mathrm{p}-\mathrm{Pb}$ and $\mathrm{pp}$ analysis are exactly the same, therefore ,only the differences are highlighted.

\subsection{Event Selection}

The proton-lead data used in this analysis are from the 2013 period (LHC13d,e,f, whose run numbers are in appendix A.2), at $\sqrt{s_{N N}}=5.02 \mathrm{TeV}$. As in pp, EMCal EMC7 triggered events were selected. Events were also required to have the primary vertex position within the interval $\left|z_{v t x}\right|<10 \mathrm{~cm}$.

\subsection{Electron-Jet Pairs Selection}

The pre-selected tracks (cuts shown in table 5.2 are paired to all the jets in the event, like in pp. The electron identification procedure is applied to these pairs, separated in the same $p_{\mathrm{T}}^{e}$ ranges $I_{p_{\mathrm{T}}^{e}}$, as in pp, namely $[3,4[\mathrm{GeV} / c,[4,6[\mathrm{GeV} / c,[6,9[\mathrm{GeV} / c$ and $[9,18[$ $\mathrm{GeV} / c$.

The obtainment of the HFe-jet $p_{\mathrm{T}, \mathrm{chjet}}$ spectra $\frac{d^{2} N^{H F e}}{d \eta d p_{\mathrm{T}, \mathrm{chjet}}}$, for away and near side $\Delta \varphi$ regions, is summarized in equation 6.1. Due to the dependence on the $p_{\mathrm{T}}^{e}$ interval $I_{p_{\mathrm{T}}^{e}}$, the HFe-jet $p_{\mathrm{T}, \text { chjet }}$ spectra must be summed for each of them (equation 6.2.

The inclusive electron contribution $J^{\text {incl. }}$ and the hadronic contribution $J^{\text {had. }}$ will be discussed in sections 7.3 and 7.4 , respectively. The non-HFe contribution $J^{\text {conv }}$. calculation and its method efficiency $\epsilon_{i n v}$. will be discussed in section 7.5. The heavy-flavour decay electron identification efficiency $\epsilon_{H F e I D}$ is described in section 7.6 . 


\subsection{Inclusive Electron-Jet Selection}

The inclusive electron-jet pairs are selected via the electron identification procedure described earlier. This procedure consists in the TPC $d E / d x$ cut in the electron region the $N_{\sigma T P C}^{e}$ region $[-1.5,3.0]$ and the selection on $E / p$ signal in the region $[0.8,1.3]$. Both selections are shown in figure 7.1, which are similiar to the ones from pp collisions.
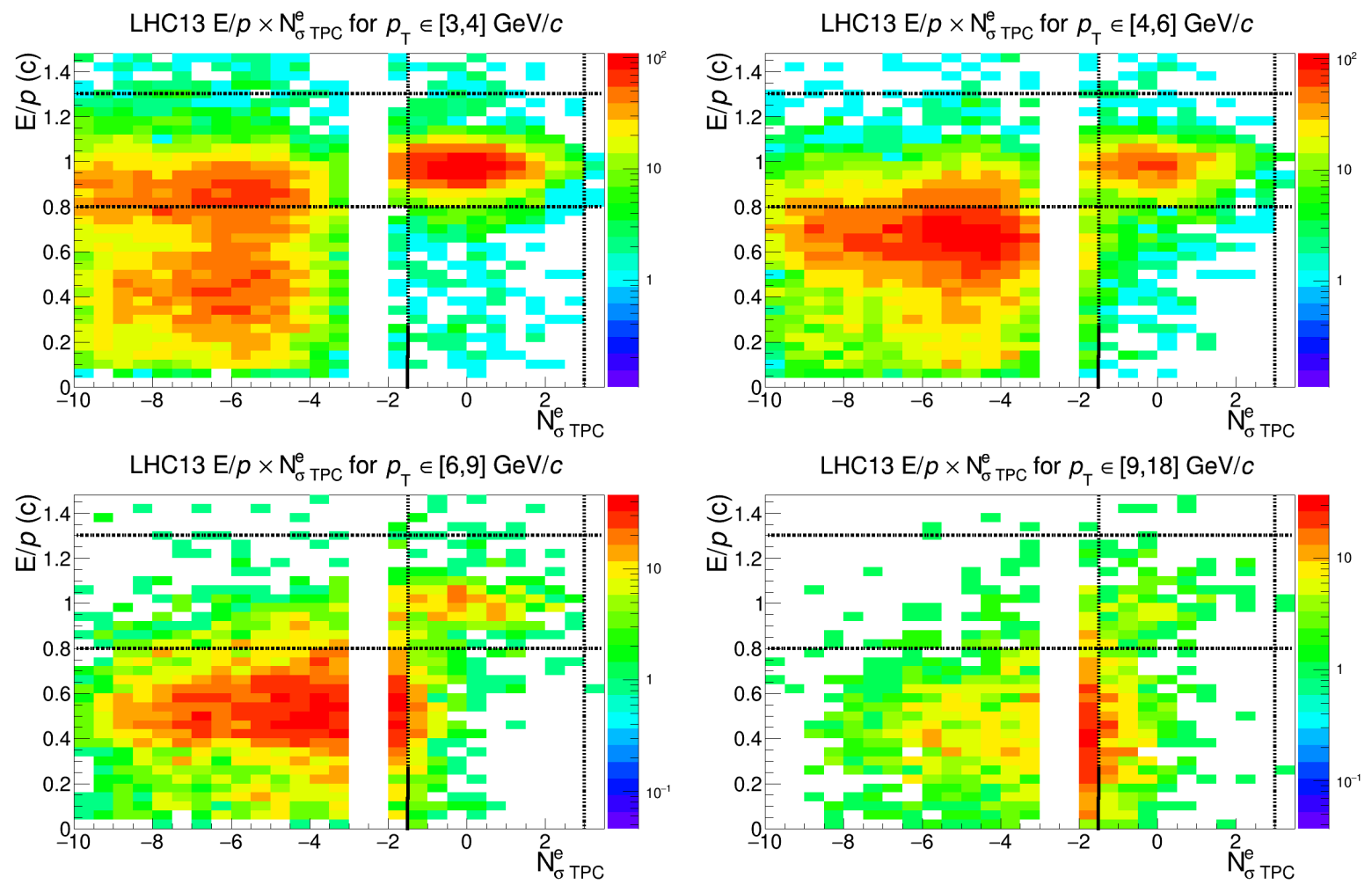

Figure 7.1: Electron-jet inclusive selection (TPC + EMCal) (p-Pb). The black, dashed lines show the selection region. Each pannel contains a different $p_{T}^{e}$ range: top left $[3,4] \mathrm{GeV} / c$, top right $[4,6] \mathrm{GeV} / c$, bottom left $[6,9] \mathrm{GeV} / c$, bottom right $[9,18] \mathrm{GeV} / c$. The empty region delimitates two different statistics regions (see appendix A.3): the left-hand side has $80 \%$ of its total statistics discarded randomly, while the right-hand side does not. The data are from $\mathrm{p}-\mathrm{Pb}$ collisions, taken in 2013 by ALICE.

The inclusive e-jet pairs must be corrected and the heavy-flavour decay electron identification efficiency $\epsilon_{H F e I D}$ taken into account. Like in pp, both corrections and efficiencies are applied in different $p_{T}^{e}$ ranges, namely $[3,4[\mathrm{GeV},[4,6[\mathrm{GeV},[6,9[\mathrm{GeV}$ and $[9,18[\mathrm{GeV}$.

\subsection{Hadronic Contamination}

The misidentified hadrons were estimated and subtracted by comparing the $E / p$ distribution of hadrons and inclusive electrons, as done in pp.

The same cuts used for e-jet pairs were applied to hadron-jet pairs except for the $N_{\sigma T P C}^{e}$, which is instead $N_{\sigma T P C}^{e} \in[-10,-3]$. The hadronic $E / p$ distribution is normalized 
so its integral in the range $E / p \in[0.24,0.74]$ is equal to the electron $E / p$ distribution in the same range. By doing so, one can calculate the relative contribution of hadrons to electrons in the region $E / p \in[0.8,1.3]$.

The values $A_{\text {norm }}$. and $f_{h}$ are calculated (according to equation 6.4 for each $p_{\mathrm{T}}^{e}$ bin (as shown in figures 7.2 and 7.4. The hadron-jet distribution $\frac{d^{2} N\left(I_{p_{\mathrm{T}}^{e}}\right)}{d \eta d p_{\mathrm{T}, \mathrm{chjet}}}$ is normalized so its integral is the correct fraction of the e-jet one. The $J^{\text {had. }}$ contribution is subtracted from the inclusive $J^{\text {incl. }}$ distribution.
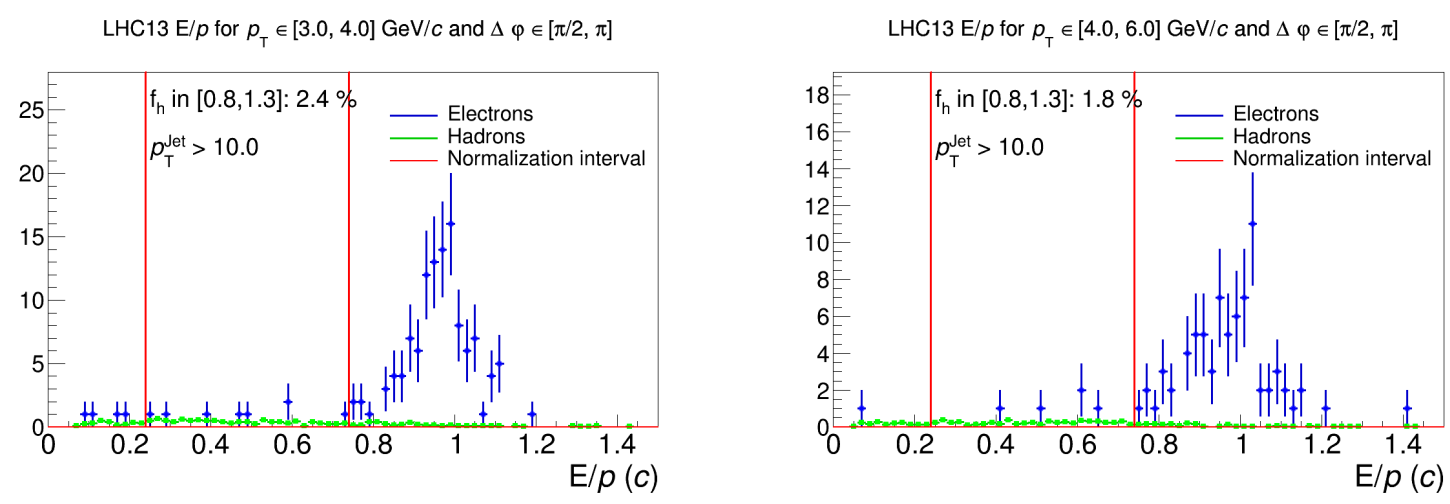

LHC13 E/p for $p_{\mathrm{T}} \in[6.0,9.0] \mathrm{GeV} / c$ and $\Delta \varphi \in[\pi / 2, \pi]$

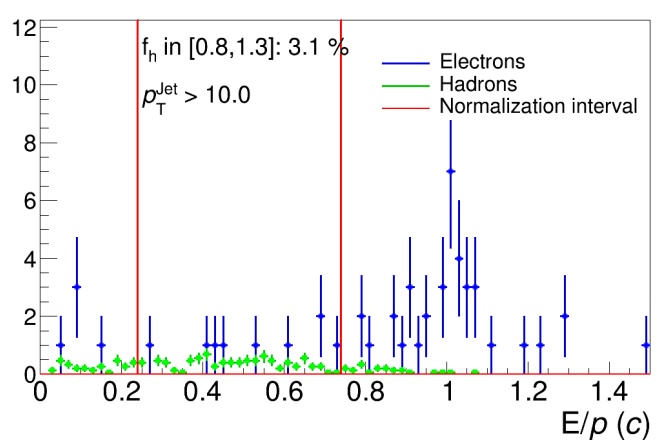

LHC13 E/p for $p_{\mathrm{T}} \in[9.0,18.0] \mathrm{GeV} / c$ and $\Delta \varphi \in[\pi / 2, \pi]$

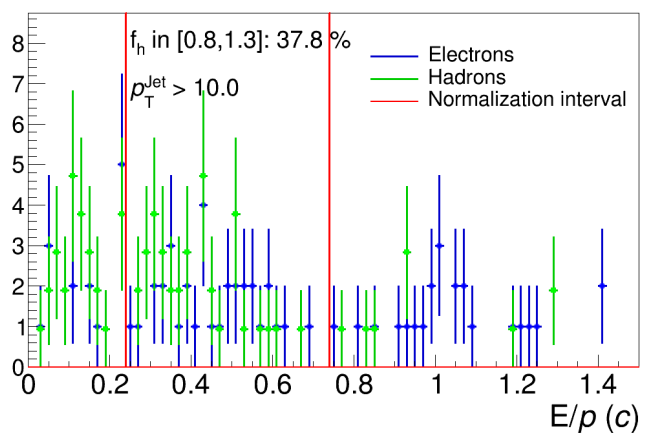

Figure 7.2: Away side Electron-Jet $E / p$ hadronic contamination. The red bars show the normalization interval used in the $A_{\text {norm }}$. calculation. The data are from $\mathrm{p}-\mathrm{Pb}$ collisions, taken in 2013 by ALICE. 
LHC13 E/p for $p_{\mathrm{T}} \in[3.0,4.0] \mathrm{GeV} / c$ and $\Delta \varphi \in[0, \pi / 10]$

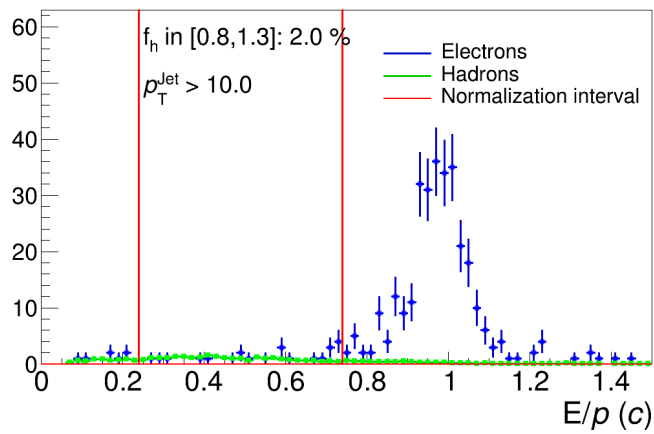

$\mathrm{LHC} 13 \mathrm{E} / p$ for $p_{\mathrm{T}} \in[6.0,9.0] \mathrm{GeV} / c$ and $\Delta \varphi \in[0, \pi / 10]$

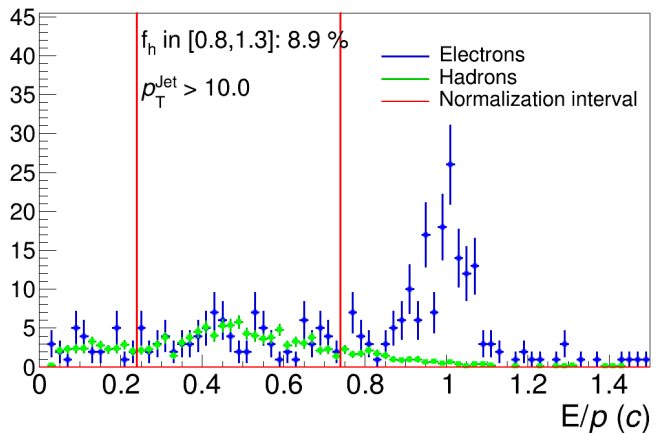

LHC13 E/p for $p_{\mathrm{T}} \in[4.0,6.0] \mathrm{GeV} / c$ and $\Delta \varphi \in[0, \pi / 10]$

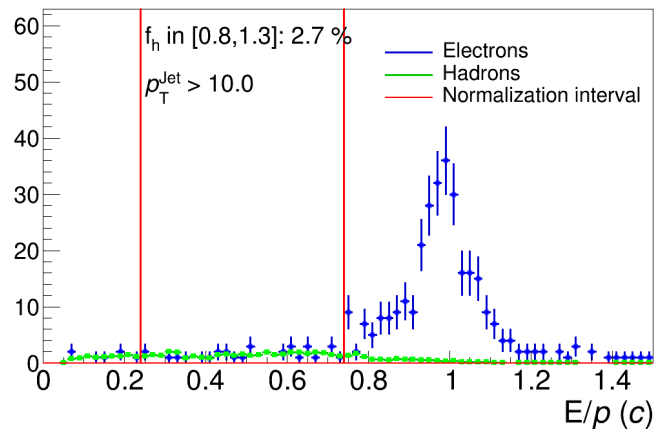

LHC13 E/p for $p_{\mathrm{T}} \in[9.0,18.0] \mathrm{GeV} / c$ and $\Delta \varphi \in[0, \pi / 10]$

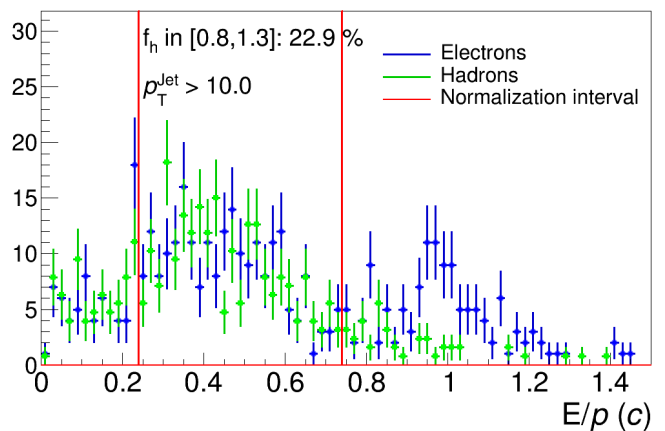

Figure 7.3: Near side Electron-Jet $E / p$ hadronic contamination. The red bars show the normalization interval used in the $A_{\text {norm. }}$. calculation. The data are from $\mathrm{p}-\mathrm{Pb}$ collisions, taken in 2013 by ALICE.

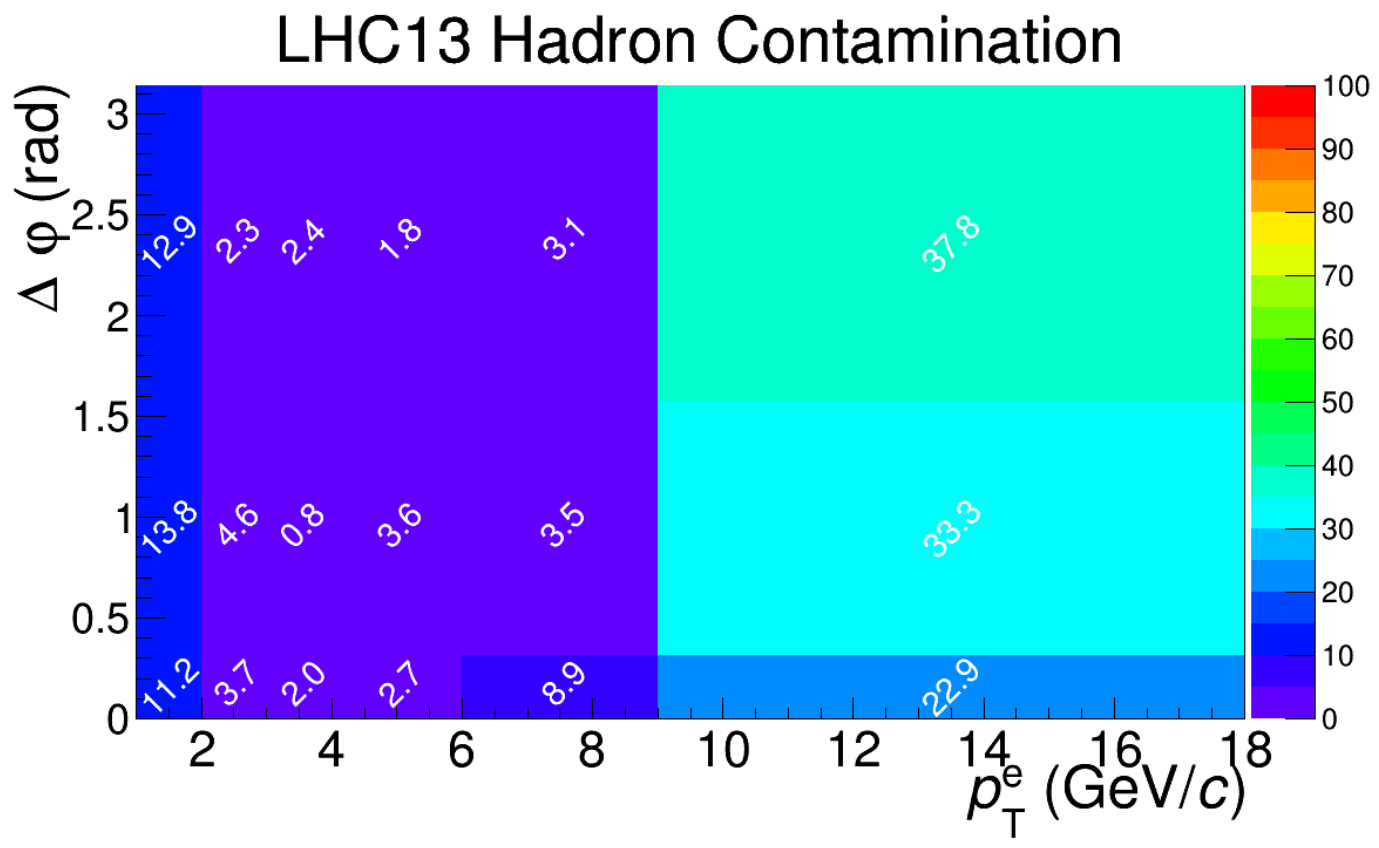

Figure 7.4: Hadronic contamination percentages $f_{h}$ for each $p_{\mathrm{T}}^{e} \times \Delta \varphi$ bin. The data are from p- $\mathrm{Pb}$ collisions, taken in 2013 by ALICE. 


\subsection{Non-HF Electron Contamination}

The last contamination to be considered is the non-heavy-flavour electron one, as explained in section 5.5. The estimate must be calculated (equation 6.5 for each $p_{T}^{e}$ bin so the efficiencies can be taken into account. Figure 7.5 shows the invariant mass distribution used in this calculation. The efficiency of the invariant mass method, shown in figure 7.6, was calculated (according to equation 6.6) using a Monte Carlo simulation provided by ALICE (LHC14b3a period).

LHC13 - Invariant Mass Distribution

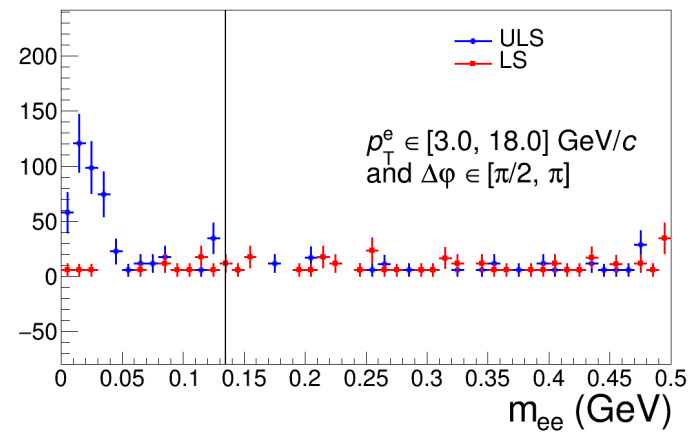

LHC13 - Invariant Mass Distribution

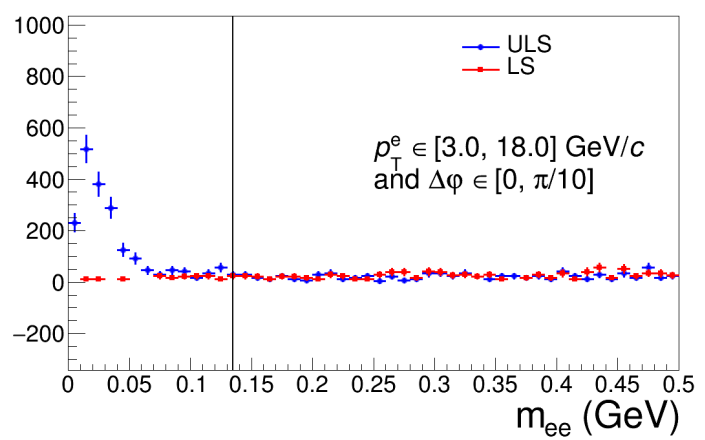

Figure 7.5: Invariant mass distribution for like-sign $e^{+} e^{-}$pairs (in red) and for unlike-sign pairs (in blue). The black bar shows the mass cut $0.135 \mathrm{GeV}$ : only the region to its left is used in the non-HF electron contribution. The left panel shows the away side and the right panel shows the near side. The data are from p-Pb collisions, taken in 2013 by ALICE.

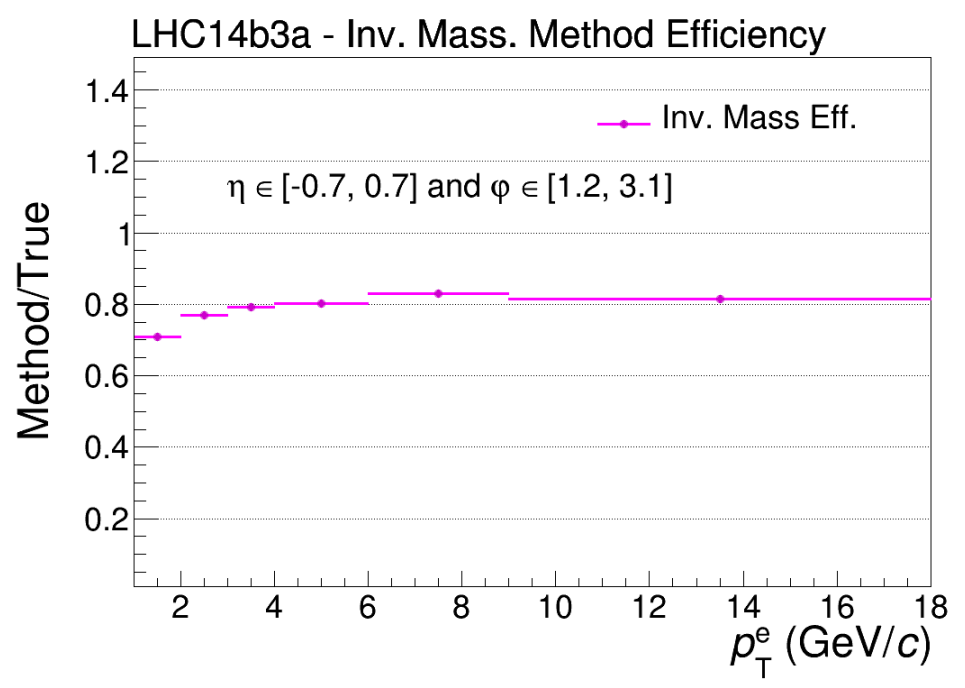

Figure 7.6: Invariant mass method efficiency. The data are from $\mathrm{p}-\mathrm{Pb}$ Monte Carlo simulations, by ALICE.

\subsection{Electron Identification Efficiency}

As for pp, the same analysis performed in data was done in the simulation, but including the generator information. The number of generated heavy-flavour decay electrons 
was compared to the number of electrons that passed the selection. The efficiency is calculated by taking their ratio and applied to the data analysis, in each $p_{T}^{e}$ interval.

The analysis in $\mathrm{p}-\mathrm{Pb}$ simulation ( $\mathrm{LHC} 14 \mathrm{~g} 3 \mathrm{~b}$ period) was performed exactly as in data. The efficiency was calculated according to the equation 6.7 and it is shown in figure 7.7 .

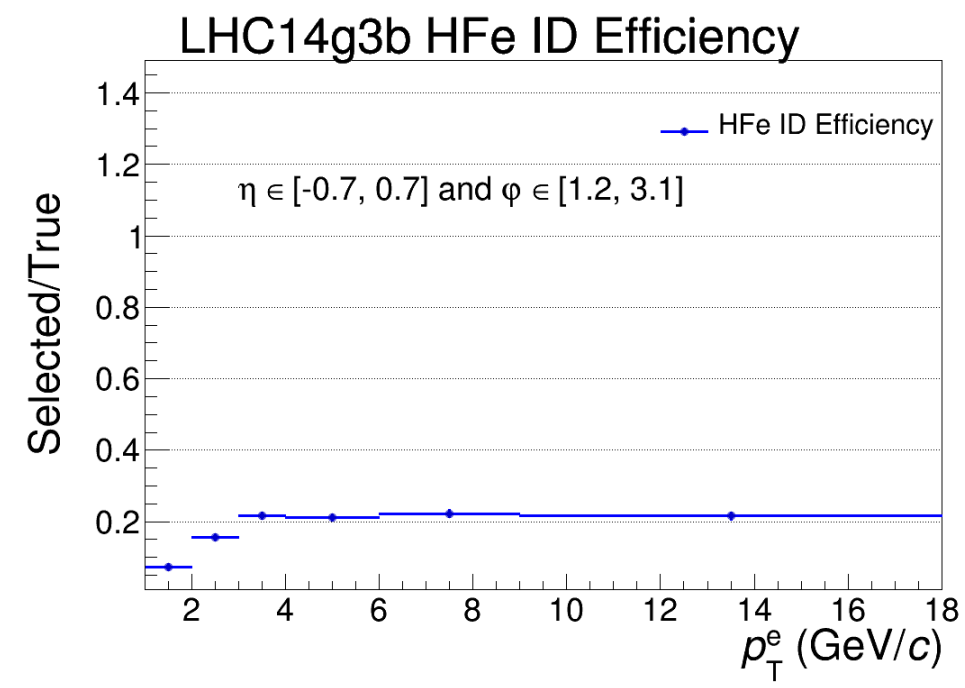

Figure 7.7: Heavy-flavour decay electron identification efficiency. The data is from an ALICE simulation (LHC14g3b period) for $\mathrm{p}-$ $\mathrm{Pb}$ collisions.

\subsection{HF Electron-Jet $\Delta \varphi$}

The angular distribution (equation 6.8) results from the same procedure as done for the jet $p_{T, \text { chjet }}$ spectrum. Figure 7.8 shows the distributions (the three contributions and the corrected one) for the $p_{T}^{e} \in[3,4[\mathrm{GeV}$ interval.

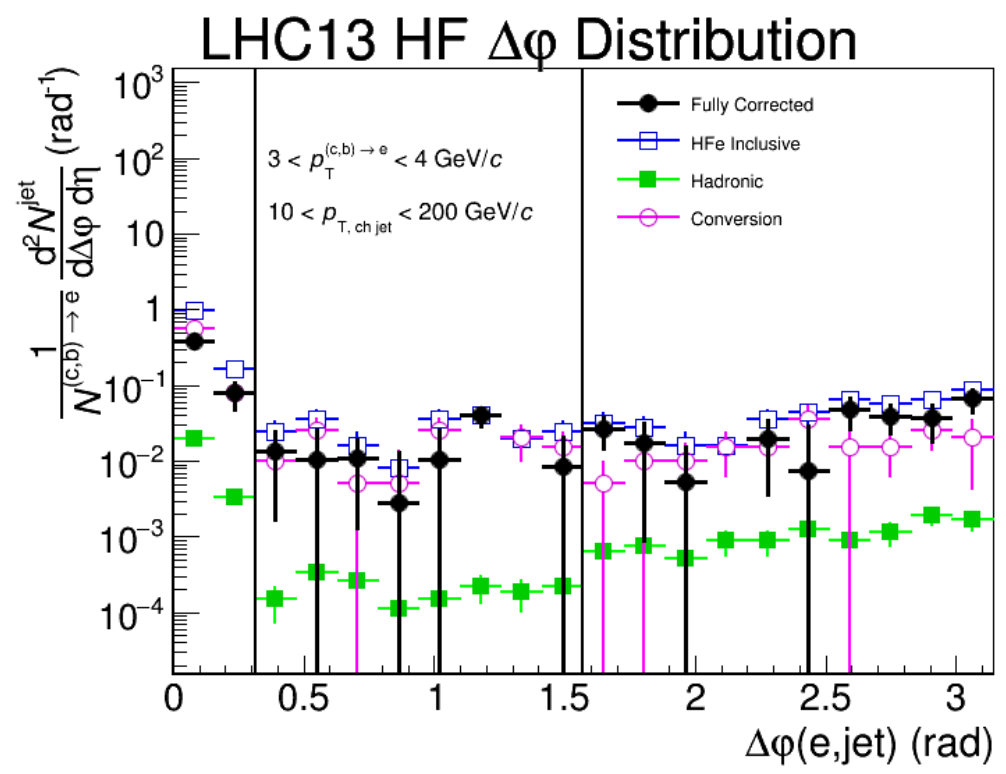

Figure 7.8: HFe-jet $\Delta \varphi$ normalized distributions: in black, the corrected one; in blue, the inclusive; the hadronic contribution is in green; and the conversion contribution is in magenta. Vertical lines are the divisions between away side, near side and middle. The data are from $\mathrm{p}-\mathrm{Pb}$ collisions. 


\subsection{Unfolding}

The standard unfolding procedure uses the RooUnfold libraries to perform a Iterative Bayesian unfolding, as in pp. The difference is the input parameter: the unfolded spectrum is output after 4 iterations. Appendix A.7 discusses the uncertainties related to the unfolding.

The response matrix corresponds to the detector response to jet measurements and the result, for away and near sides, is shown in figure 7.9 .
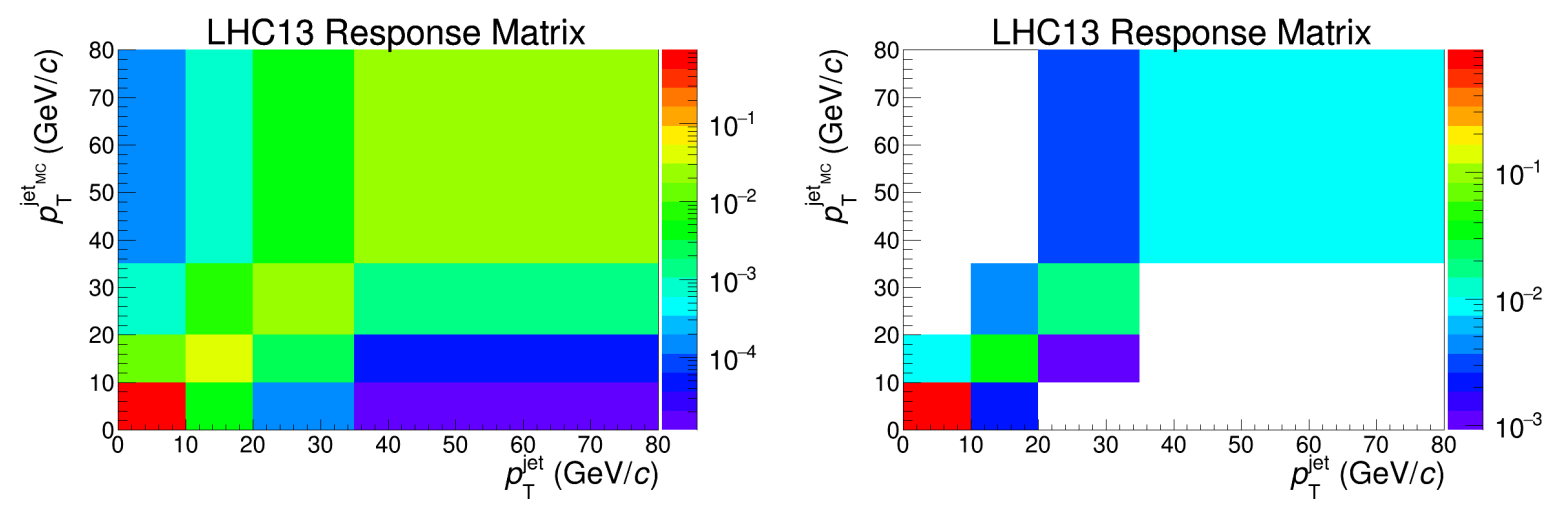

Figure 7.9: Away side response matrix is in the left-hand side. Near side response matrix is in the right-hand side. The data is from an ALICE simulation for p-Pb collisions (LHC14g3b period).

\section{Detector Response}

Using a Monte Carlo simulation information (LHC14g3b period from ALICE), the generator level jets and the reconstructed level jets were associated to each other. The maximum distance for pairing was $\Delta R_{\text {jets }}<0.2$ and the jet pairs were also associated to the electrons in the event, like in pp, so they could be divided in the three angular regions (away side, near side and middle). The efficiency of finding a generator level jet information was included in the detector response matrix, as in pp.

\subsection{HF Electron-Jet Spectrum}

All the calculated contributions - namely inclusive, hadronic and non-HF electrons are shown in figure 7.12 (left panel). They are already corrected by the efficiencies and still must be normalized

\section{Normalization}

The normalization $N_{H F e}$, for all HFe-jet observables, was calculated according to equation 6.10. The hadronic contamination fraction $f_{h}^{\text {tracks }}$ is calculated (for a single electron) 
using the $E / p$ distribution (figure 7.10). The invariant mass method is used to calculate the conversion contribution $N_{U L S}-N_{L S}$. In figure 7.11, the three contributions and the corrected spectrum are shown. The normalization $N_{H F e}$ is the integral, in the proper range, of the corrected distribution.

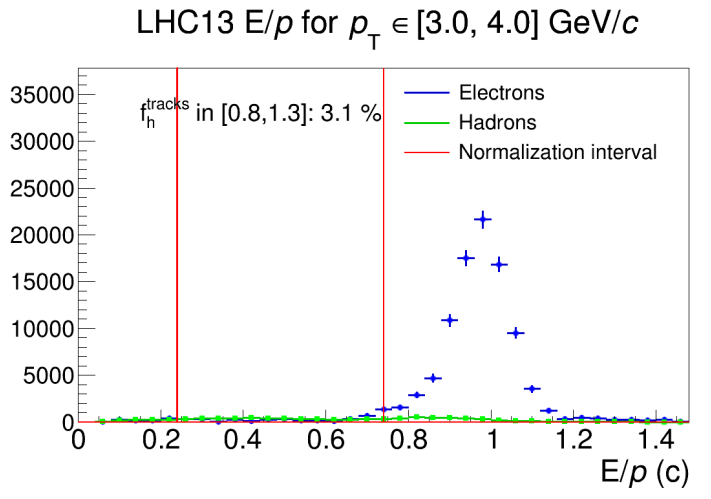

$\mathrm{LHC} 13 \mathrm{E} / p$ for $p_{\mathrm{T}} \in[6.0,9.0] \mathrm{GeV} / \mathrm{c}$

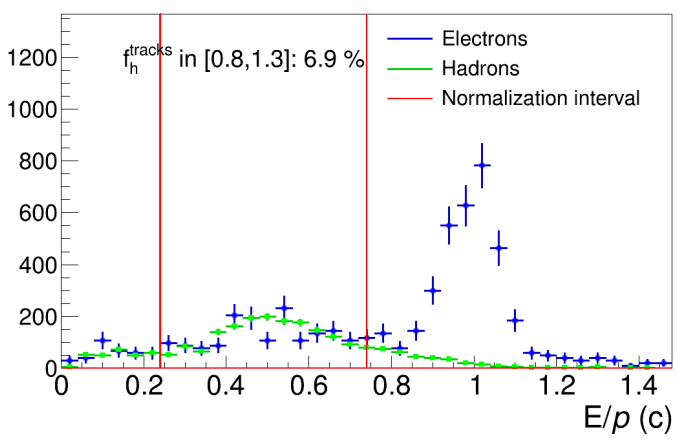

LHC13 E/p for $p_{\mathrm{T}} \in[4.0,6.0] \mathrm{GeV} / \mathrm{c}$

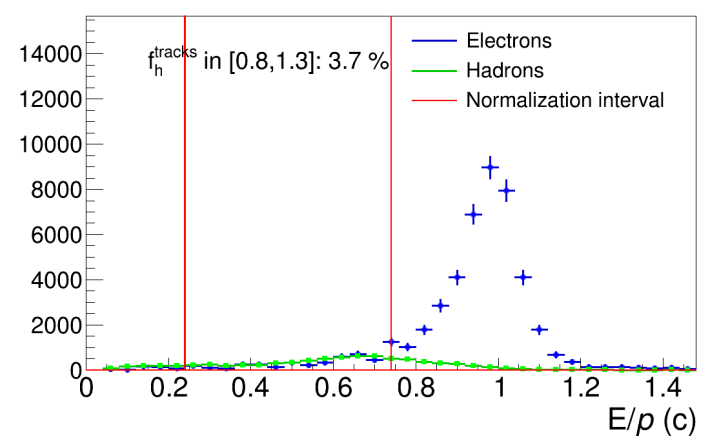

LHC13 $\mathrm{E} / p$ for $p_{\mathrm{T}} \in[9.0,18.0] \mathrm{GeV} / \mathrm{c}$

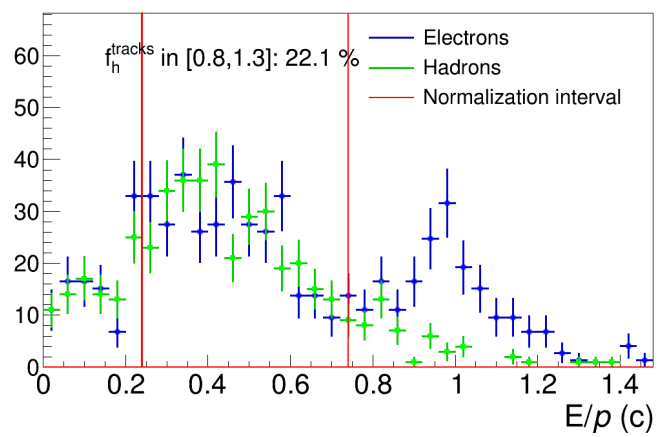

Figure 7.10: Hadronic contamination fractions for tracks only (not e-jet pairs). The blue points are the inclusive electron distribution, the green points are the estimated hadronic distribution and the bars highlight the normalization interval. Data from 2013 p-Pb collisions.

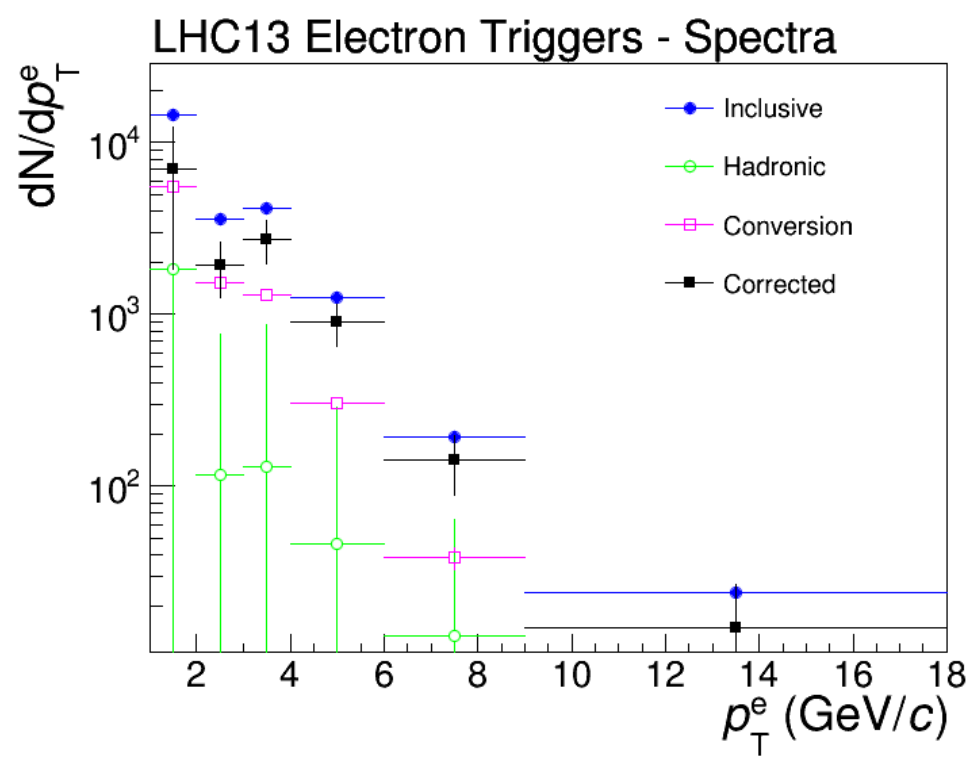

Figure 7.11: Heavyflavour electron $p_{\mathrm{T}}^{e}$ spectrum. The three non-normalized contributions are shown separately: inclusive is in blue; hadronic is in green; non-HF electrons are in magenta. The corrected spectra is in black. Data from 2013 $\mathrm{p}-\mathrm{Pb}$ collisions.

The final, corrected and unfolded spectrum, for each $\Delta \varphi$ region, are shown in figures 
7.12 and 7.13 .
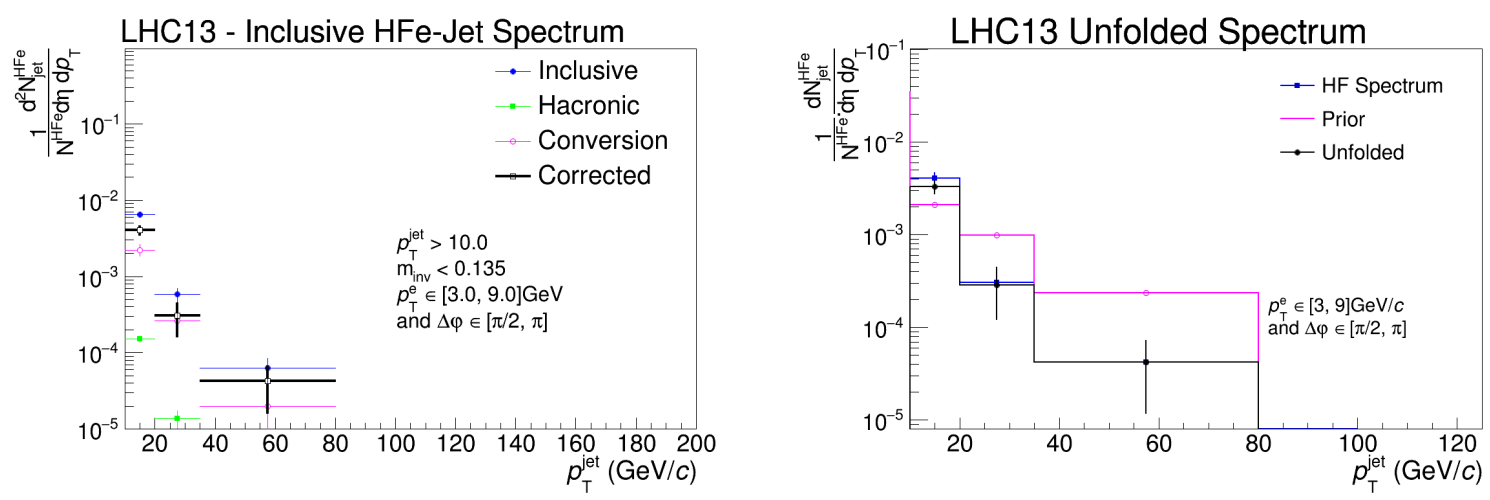

Figure 7.12: Away side: In the left panel, the normalized contributions for the e-jet distribution are shown, namely inclusive (blue), hadronic (green) and non-HF electron (magenta). The corrected one is in black. In the right panel, the unfolded spectrum is shown. The prior used in the unfolding procedure is in magenta. Data from $2013 \mathrm{p}-\mathrm{Pb}$ collisions.
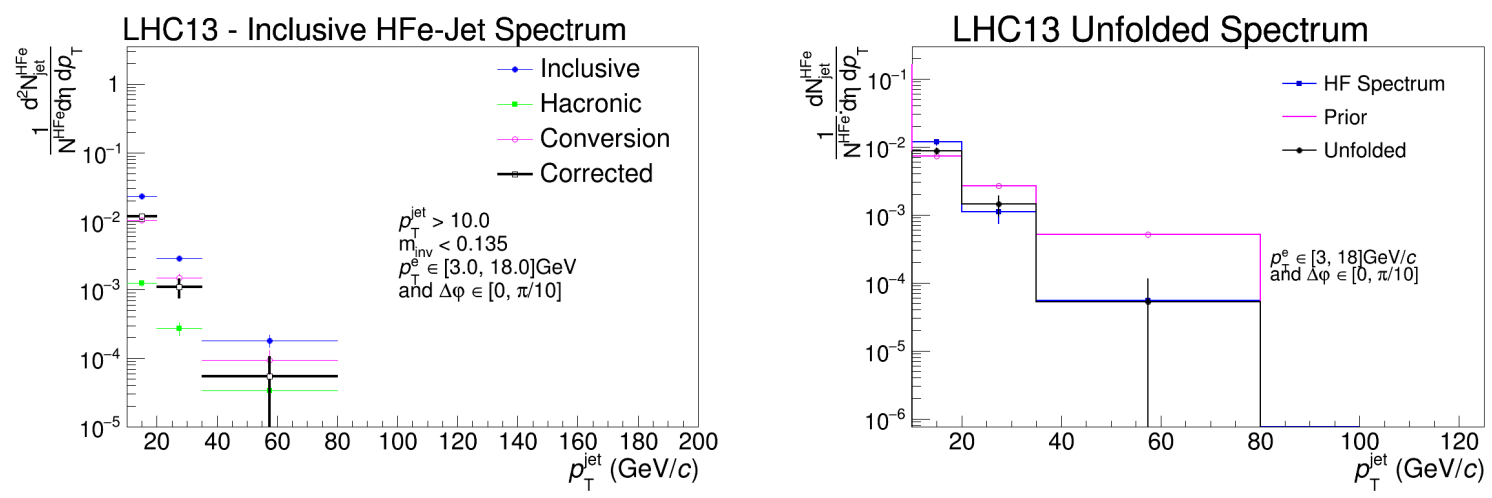

Figure 7.13: Near side: In the left panel, the normalized contributions for the e-jet distribution are shown, namely inclusive (blue), hadronic (green) and non-HF electron (magenta). The corrected one is in black. In the right panel, the unfolded spectrum is shown. The prior used in the unfolding procedure is in magenta. Data from $2013 \mathrm{p}-\mathrm{Pb}$ collisions.

\subsection{Systematic Uncertainties}

The systematic uncertainty for $\mathrm{p}-\mathrm{Pb}$ data is calculated using the same procedure used in pp. The spectra that results from different cuts (equation 6.11) are compared, and the Barlow's Method selects those that are taken into account for the systematic uncertainty calculation. The variations are summarized in table 7.1 .

The final spectrum with systematics uncertainties, for the two $\Delta \varphi$ regions, are shown in figures 7.14 and 7.15 . 


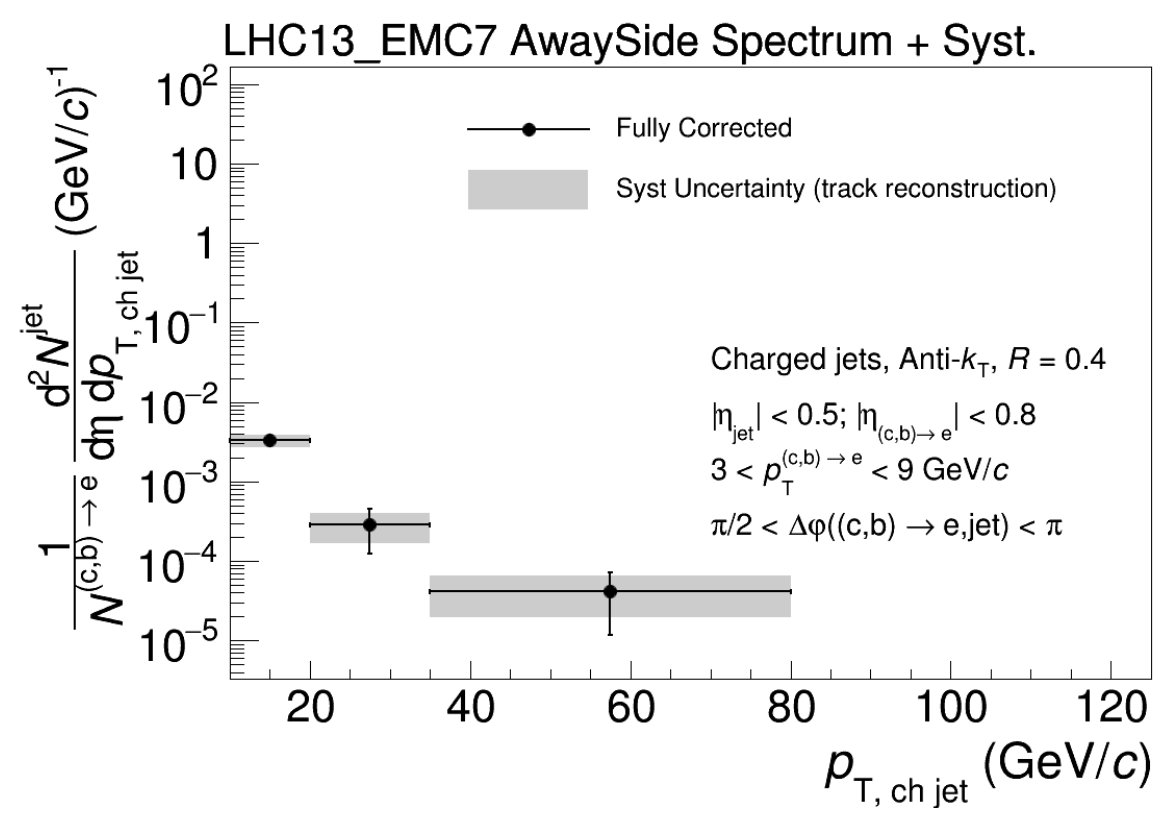

Figure 7.14: Away side: HFe-jet spectrum with systematic uncertainties.

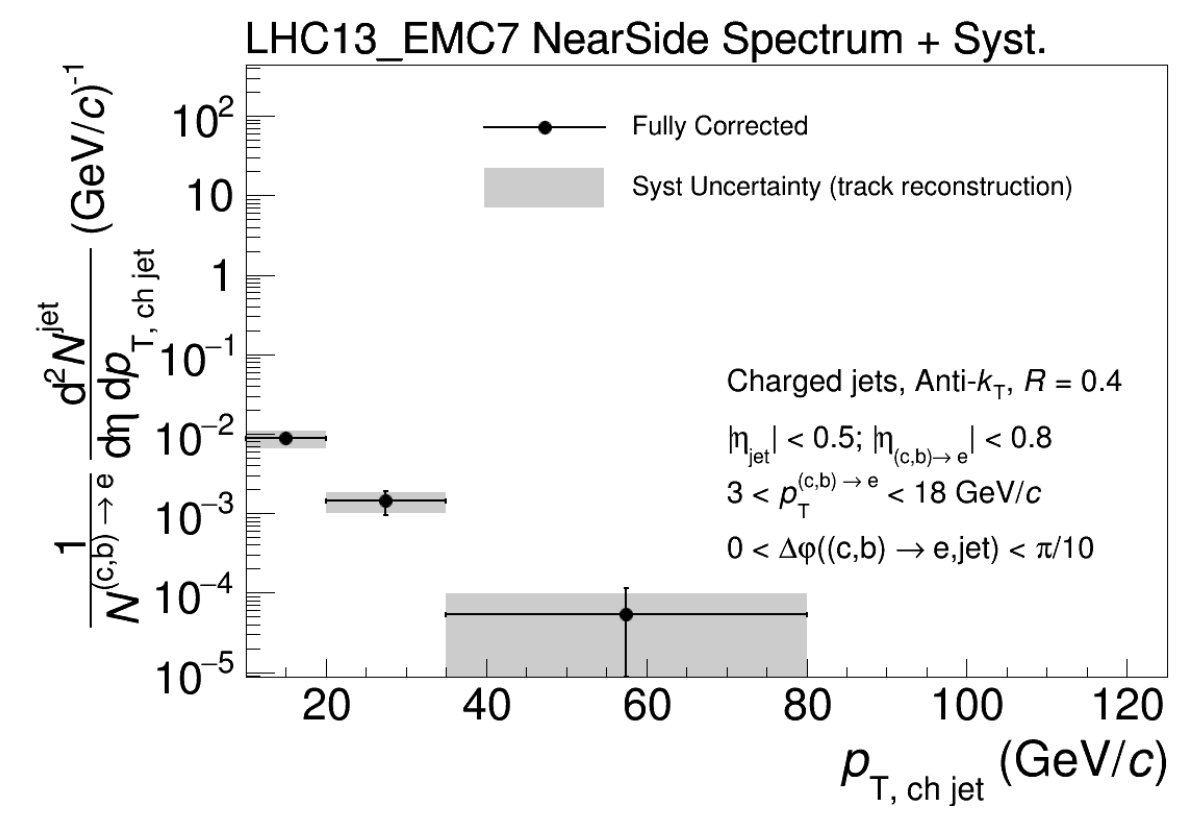

Figure 7.15: Near side: HFe-jet spectrum with systematic uncertainties. 
Table 7.1: Variation of cuts and selections for systematic uncertainty calculation in $\mathrm{p}-\mathrm{Pb}$

\begin{tabular}{|c|c|c|c|c|}
\hline Variable & Variation(s) & Standard & $\begin{array}{c}\text { Syst. Uncert. } \\
\text { Away side }\end{array}$ & $\begin{array}{c}\text { Syst. Uncert. } \\
\text { Near side }\end{array}$ \\
\hline Minimum TPC Clusters & 90,110 & 80 & & \\
\hline Min. TPC Clusters (PID) & 90,110 & 80 & $<2 \%$ & $\approx 0 \%$ \\
\hline Min. $\overline{\text { Hits }} \overline{\text { in }} \overline{\mathrm{ITS}}$ & 3,4 & 2 & $-\overline{4}$ & 54 \\
\hline $\bar{M} \overline{\text { Minimum }} \bar{N}_{\sigma T P C}^{\bar{e}}$ & $-1.0,-0.5,0.0,0 . \overline{2} 5,0.5$ & -1.5 & $<36 \%$ & $<53 \%$ \\
\hline Maximum $N_{\sigma}^{e} P_{-} P_{-} C_{-}-{ }_{-}$ & $\begin{array}{c}2.5,3.5 \\
\ldots \ldots\end{array}$ & 3.0 & & \\
\hline Minimum $E / p$ & $0.76,0.78,0.82,0.84,0.86$ & 0.80 & $<8 \%$ & $<9 \%$ \\
\hline $\begin{array}{l}\text { Maximum } E / p \\
-\bar{M}-{ }_{-}-\overline{a x}_{-}-\bar{M} \bar{M} \overline{0}\end{array}$ & $-------\frac{1}{0.2} \cdot--_{0.4}^{-----~}$ & $--\frac{1.3}{0 . \overline{3}}-$ & $--\overline{39}$ & \\
\hline$-{ }^{-} \overline{\text { Maximum }}\left|\bar{\Delta} \bar{\varphi}_{\text {match }}^{---}\right|$ & 0.03 & $0 . \overline{0} 5$ & $\approx \overline{0} \%$ & $\approx \overline{0} \%$ \\
\hline Min. $\overline{\mathrm{T}} \overline{\mathrm{P}} \overline{\mathrm{C}} \mathrm{C}$ lusters (Partner) & $-\cdots-\overline{80}-\cdots$ & $---\overline{70}--$ & $\approx \overline{0} \overline{\%}$ & $\approx \overline{0} \%$ \\
\hline Minimum $p_{\mathrm{T}}^{\bar{e}, \bar{p} a \bar{r} t \bar{n} e \bar{r}}$ & $1.0 \mathrm{G} \overline{\mathrm{eV}} / c^{-\cdots-}$ & $0.15 \mathrm{GeV} / \bar{c}$ & $\approx 0 \overline{0}$ & $<15 \%$ \\
\hline Maximum $m_{i n v}$ & $0 . \overline{100} \overline{\mathrm{GeV}} \overline{\mathrm{V}}, \overline{0.180} \overline{\mathrm{GeV}}$ & $0 . \overline{135} \overline{\mathrm{G}} \overline{\mathrm{eV}}$ & $\approx \overline{0} \%$ & $<\overline{12} \%$ \\
\hline Maximum $\left|N_{\sigma T P C}\right| \bar{e}$ (Partner) & $\overline{2} . \overline{5}^{--}$ & $--\overline{3} .0^{-}$ & $\approx 0 \%$ & $\approx 0 \overline{0}$ \\
\hline Unfolding Methò & $\overline{\mathrm{SVD}} \overline{\mathrm{S}}$ & Iterative Bayesian & & \\
\hline Unfolding Parameter $K$ & $K+1$ & $K=4$ & $<41 \%$ & $<20 \%$ \\
\hline Unfolding Prior & Power Law & Response Y-Projection & & \\
\hline
\end{tabular}




\section{Discussion}

The observables obtained from the $\mathrm{pp}$ and $\mathrm{p}-\mathrm{Pb}$ data, namely the $\Delta \varphi$ distribution and the $p_{\text {T,chjet }}$ spectrum, can be combined in order to explore the system differences, i.e. possible CNM effects. An observable similar to the Nuclear Modification Factor was chosen for assessing it.

For the near side, jets and heavy-flavour quarks (and electrons) must be angularly close. Therefore, the heavy-flavour presence makes jets more likely to have a heavy-flavour origin, with no dependence on the process that generated the event: flavour creation, flavour excitation or gluon splitting. Neverthelsess, although from hard scattering process, away side jets do not have a clear bias and may have different origins: the anti-partner from a flavour creation or excitation, or other gluon splitting products. The away and near side observables have different interpretations and their results must be analysed differently.

For a electron $p_{\mathrm{T}}^{e}$-integrated measurement, one can calculate the ratio of the jet $p_{\mathrm{T}, \mathrm{chjet}}$ spectra in $\mathrm{p}-\mathrm{Pb}$ and in $\mathrm{pp}$. For a jet $p_{\mathrm{T} \text {,chjet}}$-integrated measurement, but $p_{\mathrm{T}}^{e}$ dependent, one can calculate the yield of the near side and the away side $\Delta \varphi$ peak and take the ratio for different systems. Both observables are discussed in sections 8.2 and 8.3 .

The proper scaling of pp results to the $\mathrm{p}-\mathrm{Pb}$, due to the different energy of center of mass in their collisions, is discussed in the following section.

\subsection{FONLL Energy Scaling}

Since the pp spectrum at $\sqrt{s}=8 \mathrm{TeV}$ will be compared to p-Pb collisions at $\sqrt{s_{N N}}=$ $5.02 \mathrm{TeV}$, it must be scaled for the energy difference between the two measurements. In order to do so, the ratio of the FONLL heavy-flavour decay electrons cross sections [63, 64] for pp collisions at the two center of mass energies was taken as the scaling factor $f_{8 \rightarrow 5}$ (equation 8.1)

$$
f_{8 \rightarrow 5}\left(I_{p_{\mathrm{T}}^{e}}\right)=\frac{\int_{I_{p_{\mathrm{T}}^{e}}} \sigma_{F O N L L}^{5 \mathrm{TeV}} d p_{\mathrm{T}}^{e}}{\int_{I_{p_{\mathrm{T}}^{e}}} \sigma_{F O N L L}^{8 \mathrm{TeV}} d p_{\mathrm{T}}^{e}}
$$

where $\sigma_{F O N L L}$ is the FONLL cross section of HFe and $I_{p_{\mathrm{T}}^{e}}$ is the electron $p_{\mathrm{T}}^{e}$ interval of 
integration. The energy values $8 \mathrm{TeV}$ and $5.02 \mathrm{TeV}$ are superscribed. The details are in appendix A.8.

Figure 8.1 shows the $f_{8 \rightarrow 5}$ values. The scaling was done for each electron $p_{\mathrm{T}}^{e}$ interval and applied to the corresponding jet spectrum $J$, that were summed (equation 8.2). This was also done to the normalization $N_{H F e}$.

$$
\frac{1}{N_{H F e}^{\text {scaled }}} J^{\text {scaled }}=\frac{1}{\sum_{I_{p_{\mathrm{T}}^{e}}} f_{8 \rightarrow 5}\left(I_{p_{\mathrm{T}}^{e}}\right) \cdot N_{H F e}\left(I_{p_{\mathrm{T}}^{e}}\right)} \sum_{I_{p_{\mathrm{T}}^{e}}} f_{8 \rightarrow 5}\left(I_{p_{\mathrm{T}}^{e}}\right) \cdot J\left(I_{p_{\mathrm{T}}^{e}}\right)
$$

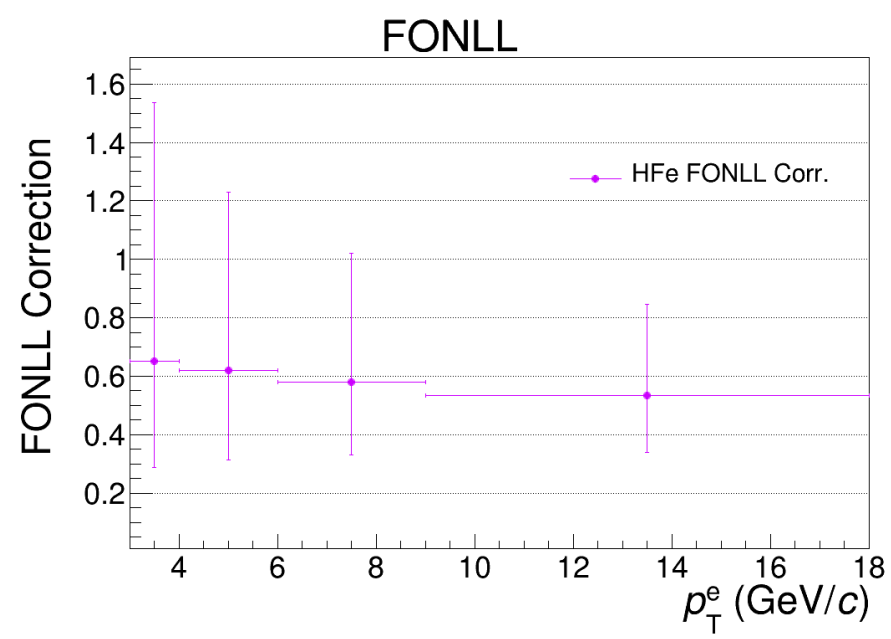

Figure 8.1: FONLL 63, 64] correction for the energy scale $f_{8 \rightarrow 5}$ is the ratio between the heavy-flavour decay electrons cross section in pp collisions at $5.02 \mathrm{TeV}$ and $8 \mathrm{TeV}$.

\section{$8.2 \quad p_{\mathrm{T}}^{e}$-inclusive $I_{p P b}$}

The away side and the near side jet $p_{\mathrm{T}, \text { chjet }}$ spectra may have some background that is not directly related to a hard process. In order to subtract such background, a new spectrum is generated assuming that it contains only background jets. The chosen background spectrum $\frac{d^{2} N^{j e t, \text { Middle }}}{d \eta d p_{\text {T,chjet }}}$ is the one obtained by the same method of this analysis (detailed previously in chapters 6 and 7, and also in appendix A.4) but selecting the angular correlation in the range $\Delta \varphi \in[\pi / 10, \pi / 2]$. The background subtracted spectra $\left(\Delta_{\text {Side }}\right)$ is given by equation 8.3 :

$$
\Delta_{\text {side }}=\frac{1}{N_{H F e}^{\text {Side }}} \frac{d^{2} N^{\text {jet }, \text { Side }}}{d \eta d p_{\mathrm{T}, \text { chjet }}}-\frac{A_{\text {side }}^{\complement}}{N_{H F e}^{M i d d l e}} \frac{d^{2} N^{j e t, \text { Middle }}}{d \eta d p_{\mathrm{T}, \text { chjet }}}
$$

where side is either away or near, and $A_{\text {side }}^{<}$is the normalization calculated by scaling the angular coverage of each measurement $\left(A_{\text {near }}^{\complement}=\frac{1}{4}\right.$ and $\left.A_{\text {away }}^{\measuredangle}=\frac{5}{4}\right)$. The $\Delta_{\text {side }}$ for $\mathrm{p}-\mathrm{Pb}$ and pp collisions, whose systematic uncertainties are shown in appendix A.5, can be used to calculate the $I_{P P b}$ (equation 8.4).

$$
I_{p P b}=\frac{\Delta_{\text {Side }}^{p P b}}{\Delta_{\text {Side }}^{p p}}
$$


Figures 8.2 and 8.3 show the $I_{p P b}$ for the away side and the near side, respectively. Systematic uncertainties were combined by adding them quadratically (equation 8.5).

$$
\frac{\sigma_{\text {Sys. }}^{\text {Final }}}{I_{p P b}}=\sqrt{\left(\frac{\sigma_{\text {Sys. }}^{p p}}{\Delta^{p p}}\right)^{2}+\left(\frac{\sigma_{\text {Sys. }}^{p P b}}{\Delta^{p P b}}\right)^{2}}
$$

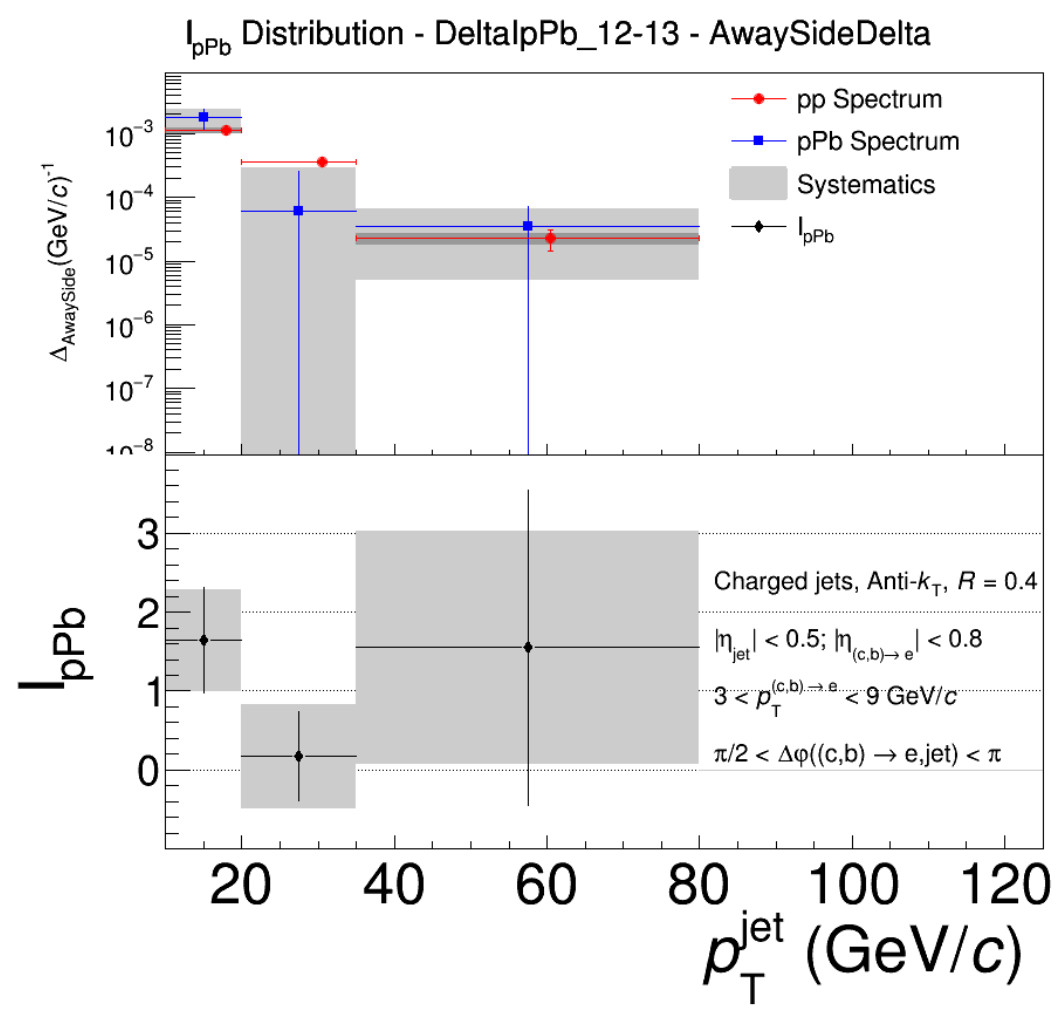

Figure 8.2: Away side $I_{p P b}$. In the top panel, the red points are the pp spectrum (displaced by $3 \mathrm{GeV} / c$ ); the blue points are the p-Pb spectrum. In the bottom panel, the ratio between the spectra, i.e. the $I_{p P b}$ is shown. The gray shade are the systematic uncertainties.

The result for $I_{p P b}$ is compatible with the unity, considering the uncertainties. This indicates that the selected jets, i.e. jets correlated to heavy-flavour decay electrons, do not have their $p_{\mathrm{T} \text {,chjet }}$ spectrum modified by the presence of a nucleus ( $\mathrm{p}-\mathrm{Pb}$ collisions), when compared to pp collisions. The compatibility with the unity can be observed for both $\Delta \varphi$ intervals, which suggests that jets with a heavy-flavour only origin (near side) and jets with a mixed heavy-flavour and gluon origins (away side) are affected similarly, although the large uncertainties do not allow futher conclusions.

The main sources of systematic uncertainties are the unfolding procedure, the $N_{\sigma T P C}^{e}$ selection and the $M 20$ selection. In order to understand their contribution, one has to further study how they affect the measurement. The statistic uncertainties can be reduced by acquiring more data. 


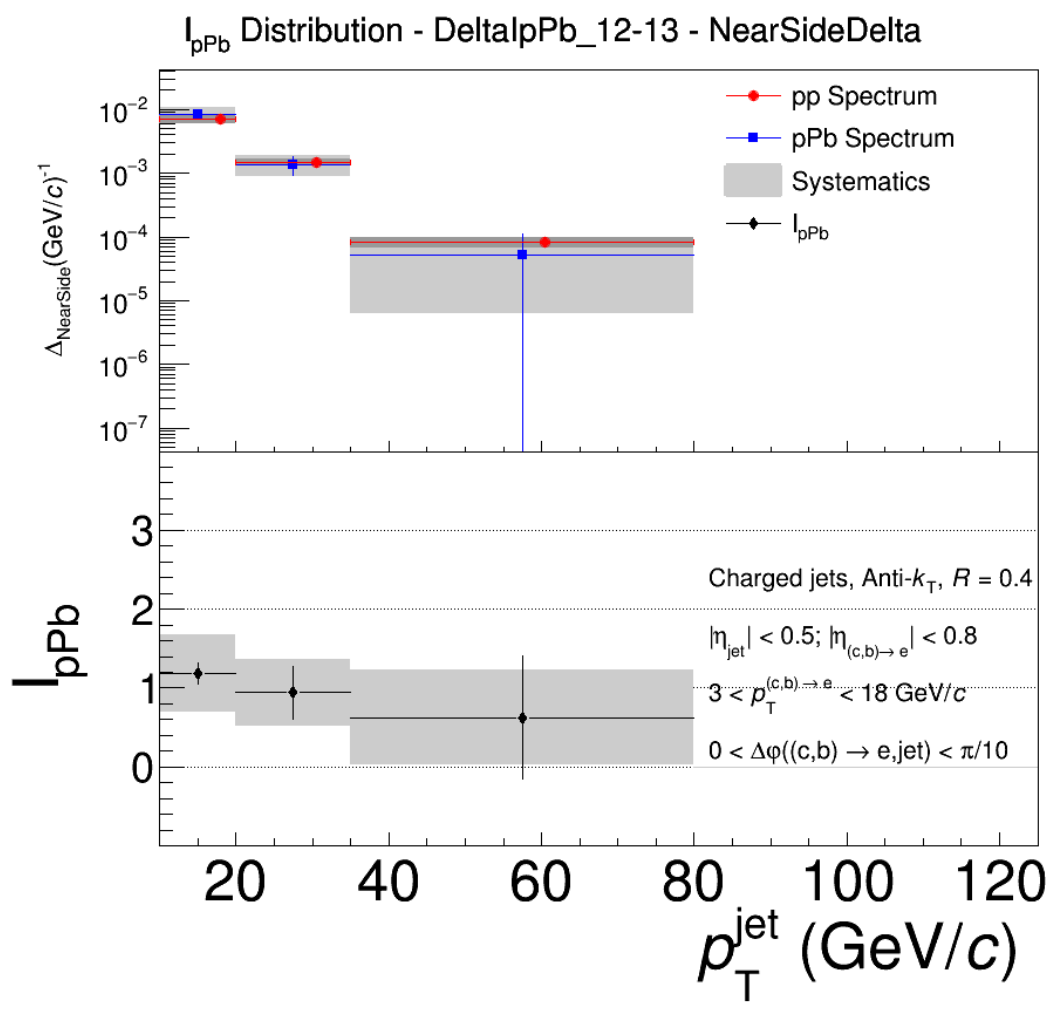

Figure 8.3: Near side $I_{p P b}$. In the top panel, the red points are the pp spectrum (displaced by $3 \mathrm{GeV} / c$ ); the blue points are the $\mathrm{p}-\mathrm{Pb}$ spectrum. In the bottom panel, the ratio between the spectra, i.e. the $I_{p P b}$ is shown. The gray shade are the systematic uncertainties.

\section{3 $p_{\mathrm{T}, \mathrm{ch} \text { jet}}$-inclusive $I_{p P b}^{Y \text { ield }}$}

Another observable that can be compared between the systems are the $\Delta \varphi$ distributions. In order to quantify such comparison, a function $f^{F i t}$ (equation 8.6),

$$
f^{F i t}(\Delta \varphi)=\text { constant }+A_{\text {near }} e^{\frac{(\Delta \varphi-0)^{2}}{\sigma_{\text {near }}^{2}}}+A_{\text {away }} e^{\frac{(\Delta \varphi-\pi)^{2}}{\sigma_{a w a y}^{2}}}
$$

composed of a pedestal and a Gaussian for each peak, was fitted to this angular distribution. This was performed for different intervals $I_{p_{\mathrm{T}}^{e}}$ in order to investigate the evolution with $p_{\mathrm{T}}^{e}$ of such distribution (figure 8.4).

The yield is taken as the Gaussian area, i.e. $\sqrt{2 \pi}\left|\sigma_{\text {side }}\right| \cdot A_{\text {side, }}$, side being either away or near. A spectrum $\Delta_{\text {side }}^{\text {ield }}$ is constructed from the yields in each $p_{\mathrm{T}}^{e}$ bin (equation 8.7).

$$
\Delta_{\text {side }}^{Y i e l d}\left(I_{p_{\mathrm{T}}^{e}}\right)=\sqrt{2 \pi}\left|\sigma_{\text {side }}\left(I_{p_{\mathrm{T}}^{e}}\right)\right| \cdot A_{\text {side }}\left(I_{p_{\mathrm{T}}^{e}}\right)
$$

The ratio $I_{p P b}^{Y \text { ield }}$ (equation 8.8 between the $\Delta_{\text {side }}^{\text {Yield }}$ spectra for $\mathrm{pp}$ and $\mathrm{p}-\mathrm{Pb}$ is shown in figure 8.5, and compared for both away side and near side.

$$
I_{p P b}^{Y i e l d}\left(I_{p_{\mathrm{T}}^{e}}\right)=\frac{\Delta_{\text {Side }}^{\text {Yield,pPb}}\left(I_{p_{\mathrm{T}}^{e}}\right)}{\Delta_{\text {Side }}^{\text {Yieldpp }}\left(I_{p_{\mathrm{T}}^{e}}\right)}
$$



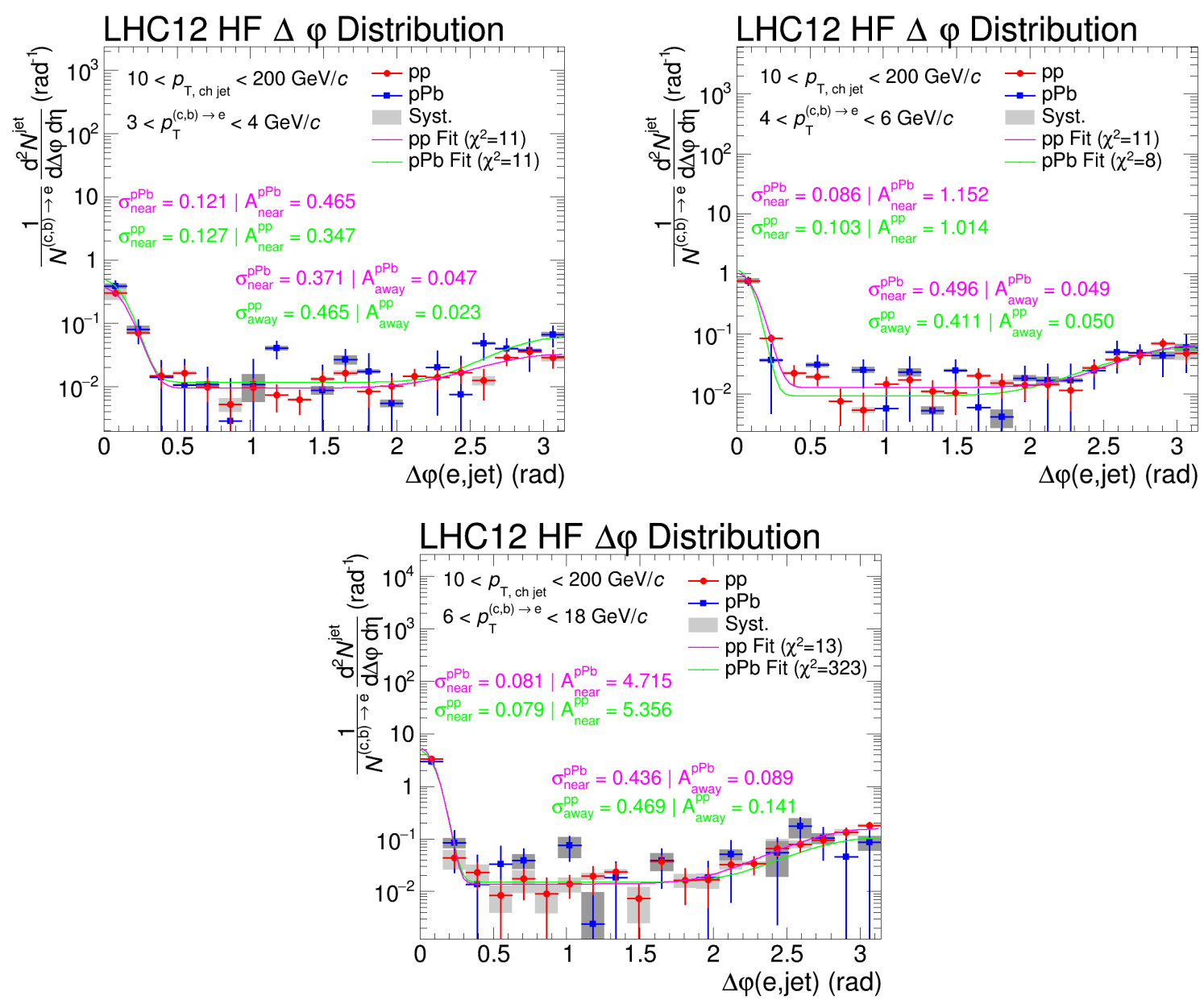

Figure 8.4: $\Delta \varphi$ distribution comparison between $\mathrm{pp}$ and $\mathrm{p}-\mathrm{Pb}$ data. The red points are the $\mathrm{pp}$ distribution; the blues ones are the $\mathrm{p}-\mathrm{Pb}$ one. The green line is the fit to $\mathrm{pp}$, and the magenta is the fit to $\mathrm{p}-\mathrm{Pb}$. Three different $p_{\mathrm{T}}^{e}$ ranges were used: top right, $p_{\mathrm{T}}^{e} \in[3,4] \mathrm{GeV} / c$, top left $p_{\mathrm{T}}^{e} \in[4,6] \mathrm{GeV} / c$ and top bottom $p_{\mathrm{T}}^{e} \in[6,18] \mathrm{GeV} / c$.

$I_{p P b}^{Y i e l d}$ values are compatible with the unity, considering the uncertainties. This indicates that the quantity of jets correlated to heavy-flavour decay electrons is not modified by the presence of a nucleus ( $\mathrm{p}-\mathrm{Pb}$ collisions), when compared to pp collisions. Although the large uncertainties lead to inconclusive results, they suggest, for both away and near sides, that there is a minimum or none dependence on electron $p_{\mathrm{T}}^{e}$. 


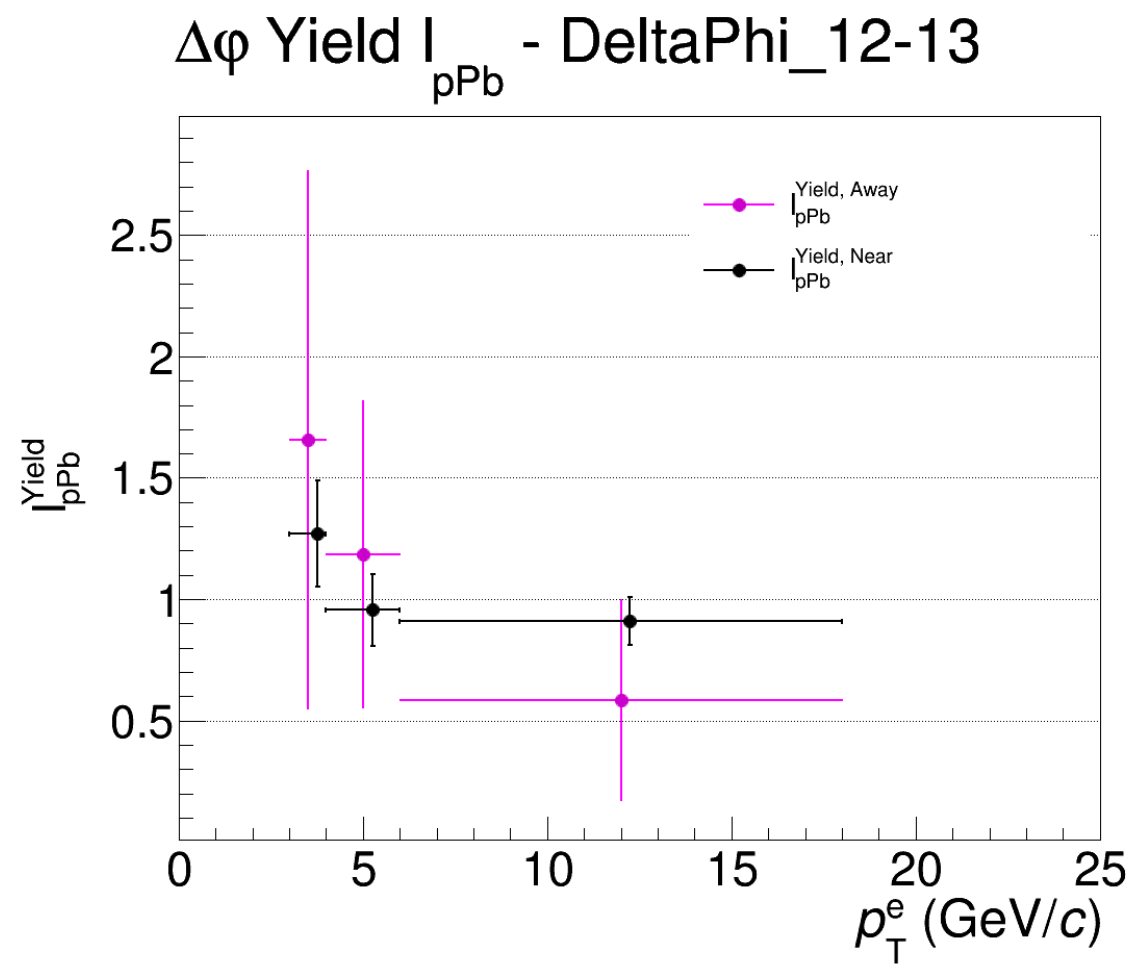

Figure 8.5: $I_{p P b}^{Y \text { ield }}$ calculated from $\Delta \varphi$ as described in the text. The magenta distribution is the away side $I_{p P b}^{Y \text { ield }}$ and the black (displaced by $0.25 \mathrm{GeV} / c$ ) is the near side one. 


\section{Conclusions}

This thesis showed the first measurement of jet spectra correlated with heavy-flavour decay electrons, the HFe-jet distribution. The jets were reconstructed using Fastjet's [8] anti $-k T$ algorithm, with parameter $R=0.4$ and minimum $p_{T, c h j e t}$ of $10 \mathrm{GeV} c$. Electrons were selected using TPC energy loss identification and EMCal energy information. They were identified as trajectories with $-1.5<N_{\sigma T P C}^{e}<3$ and $0.8<E / p<1.3$.

Electrons and jets were combined and corrected for two sources of contamination. The hadronic contamination was estimated through the $E / p$ distributions for hadrons only $\left(-10<N_{\sigma T P C}^{e}<-3\right)$ and for inclusive electrons. The non-HF electron contamination was estimated via the invariant mass method. The resulting jet $p_{\mathrm{T}, \mathrm{chjet}}$ spectrum was unfolded, using the RooUnfold [61] libraries, to subtract detector and background effects.

In order to explore possible uses of a heavy-flavour decay electron-jet pairs, two observables were proposed: $I_{p P b}$ and $I_{p P b}^{Y \text { ield }}$. The first one assesses the jet $p_{\mathrm{T}, \mathrm{chjet}}$ dependence, by taking the ratio $I_{P P b}$ of the background subtracted jet $p_{\mathrm{T}, \text { chjet }}$ spectra $\Delta_{\text {side }}$ for pp and $\mathrm{p}-\mathrm{Pb}$, after both away and near side selection. The other observable assesses the electron $p_{\mathrm{T}}^{e}$ dependence by comparing the away and near side peaks in the $\Delta \varphi$ distribution. The yields of the peaks, for each electron $p_{\mathrm{T}}^{e}$ range, were extracted from Gaussian fits to the $\Delta \varphi$ distributions. The observable $I_{p P b}^{Y i e l d}$ is the ratio of the yield values as a function of the electron $p_{\mathrm{T}}^{e}$, for the away and near side regions. All four, namely $I_{p P b}^{A w a y}, I_{p P b}^{\text {Near }}, I_{p P b}^{Y i e l d, A w a y}$ and $I_{p P b}^{Y i e l d, N e a r}$ are compatible with the unity. However, it is important to be cautious with the conclusions, given the large values of the systematic and statistic uncertainties.

Combining the two results, one may say that the heavy-flavour decay electron and the jet distributions in $\mathrm{p}-\mathrm{Pb}$ do not change in comparison with $\mathrm{pp}$. This agrees with the expectations [24, 25] of a $I_{p P b} \approx 1$, that indicates that heavy-flavour quarks lose energy similarly in pp and p-Pb collisions, as no QGP-like medium is formed, even though collective behaviours have been observed [26, 27].

Since these are new observables, there is no theoretical model that is directly comparable to it. In addition, both statistical and systematic uncertainites are large and the comparison to several non-specific models would lead to compatible results, although without any stronger conclusions.

There are many perspectives to improve this work, giving that this is the first attempt of a measurement of this observable. An improvement towards a bias of heavy-flavour 
origin would be to extend the jet selection in the away side. Jet tagging via heavy-flavour decay products (e.g. D-mesons, heavy-flavour electrons) can lead to an observable that has little effect of background hard scattering process, namely gluon splitting, flavour creation or flavour excitation. However, any further selection requires more data in order to have statistically relevant results. 


\section{A Appendix}

\section{A.1 Kinematic Variables}

\section{Transverse Momentum}

In cilindrical coordinates (figure A.1), one can project the momentum $p$ into the $x y$ plane transverse momentum $p_{\mathrm{T}}$ (equation A.1). This information is not spacially complete and must be complemented with the value of $\eta$.

$$
p_{T}=\sqrt{p^{2}-p_{z}^{2}}
$$

\section{Pseudorapidity $\eta$}

$\eta$ is a geometric variable related to the polar angle $\theta$ (figure A.1) according to the equation A.2.

$$
\eta=-\ln \left[\tan \left(\frac{\theta}{2}\right)\right]
$$

\section{Rapidity $y$}

$y$ relates to $\eta$ but is not only geometrical (equation A.3). For high energy particles, $\eta \approx y$ since $E \approx p$ and $p_{z} / p=\cos (\theta)=\cos ^{2}\left(\frac{\theta}{2}\right)-\operatorname{sen}^{2}\left(\frac{\theta}{2}\right)$.

$$
y=\frac{1}{2} \ln \left(\frac{E+p_{z}}{E-p_{z}}\right)
$$




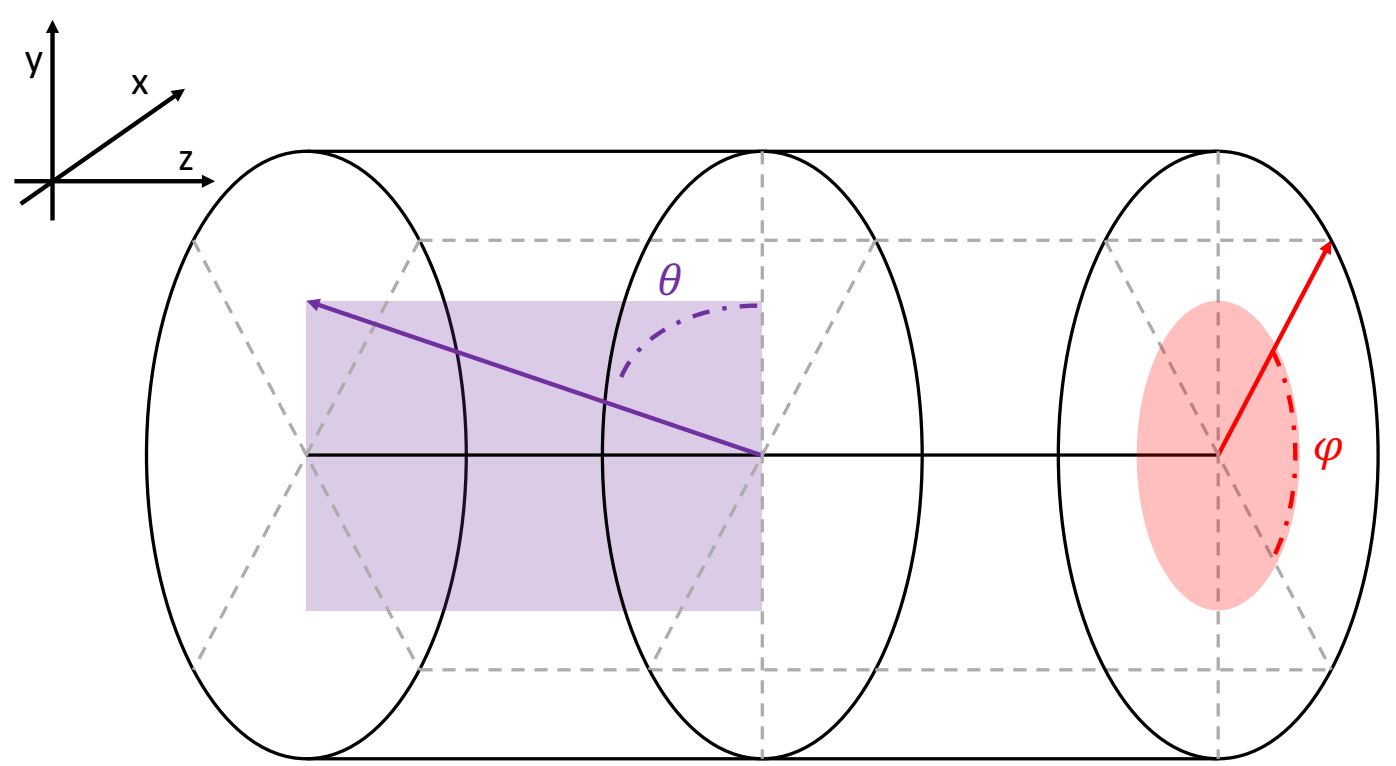

Figure A.1: Geometric variables often used in this analysis. The red region represents the face of the cylinder, where the $\varphi$ angle is measured. The purple region is the plane that divides the cylinder in two equal longitudinal halves, where the $\theta$ angle and the pseudorapidity $\eta$ are measured. 


\section{A.2 Data Samples and Code in AliROOT}

The used data sets (periods) were LHC12c,d,e,f,g,h,i $\left(1.82 \times 10^{7}\right.$ events); LHC13d,e,f $\left(8.66 \times 10^{5}\right.$ events). The used Pythia simulation (parameters defined by ALICE) data sets (periods) were LHC16a1c; LHCc14g3b; LHC1512b2; and LHC14b3a. They are divided in some recording sets of events called runs. The lists of runs, for each period, are the following:

- LHC12c: 182692, 182691, 182687, 182684, 182325, 182324, 182323, 182322, 182302, 182300, 182299, 182297, 182295, 182289, 182207, 182111, 182110, 182106, 182023, 182022, 182018, 182017, 180720, 180719, 180717, 180716, 180569, 180567, 180566, 180564, 180562, 180561, 180517, 180515, 180510, 180507, 180501, 180500, 180230, 180201, 180133, 180132, 180131, 180130, 180129, 180127, 180044, 180042, 180000, 179920, 179919, 179918, 179917, 179916, 179858, 179837, 179806, 179803, 179802, 179796, 179639, 179638, 179621, 179618

- LHC12d: 186320, 186319, 186208, 186205, 186167, 186165, 186164, 186163, 186084, 186083, 185375, 185371, 185363, 185362, 185361, 185360, 185359, 185356, 185351, 185350, 185349, 185303, 185302, 185300, 185299, 185296, 185293, 185292, 185291, 185289, 185284, 185282, 185221, 185217, 185208, 185206, 185203, 185198, 185196, $185189,185164,185160,185157,185134,185132,185127,185126,185116,185031$, 185029, 184988, 184987, 184967, 184964, 184938, 184933, 184786, 184784, 184687, 184682, 184678, 184673, 184389, 184383, 184371, 184215, 184209, 184208, 184188, 184138, 184137, 184135, 184132, 184131, 184127, 184126, 183938, 183937, 183936, 183935, 183934, 183933, 183932, 183916, 183913

- LHC12e: 186389, 186388, 186387, 186386, 186365

- LHC12f: 188123, 188108, 188101, 187796, 187791, 187785, 187749, 187744, 187739, 187698, 187656, 187654, 187627, 187624, 187623, 187510, 187489, 187488, 187487, 187341, 187340, 187339, 187203, 187202, 187152, 187151, 187150, 187149, 187146, 187143, 186992, 186990, 186969, 186966, 186939, 186938, 186937, 186851, 186811, 186692, 186690, 186689, 186688, 186668

- LHC12g: 188503, 188500, 188499, 188447, 188446, 188444, 188443, 188442

- LHC12h: 192732, 192731, 192729, 192535, 192534, 192510, 192505, 192499, 192492, 192468, 192461, 192453, 192417, 192415, 192349, 192348, 192347, 192246, 192205, 192202, 192201, 192200, 192199, 192197, 192194, 192177, 192174, 192172, 192141, 192140, 192136, 192128, 192075, 192073, 192072, 192004, 191248, 191247, 191245, 191244, 191242, 191234, 191232, 191231, 191229, 191227, 190307, 190305, 190303, 190216, 190215, 190214, 190212, 190210, 190150 
- LHC12i: 193184, 193152, 193151, 193150, 193051, 193049, 193047, 193014, 193011, 193010, 193008, 193007, 193005, 193004, 192824, 192822, 192820, 192779, 192775, 192772

- LHC13d: 195681, 195682, 195720, 195721, 195722, 195723, 195724, 195725, 195726, 195727, 195760, 195761, 195765, 195767, 195783, 195787, 195826, 195827, 195829, 195830, 195831, 195867, 195869, 195871, 195872, 195873

- LHC13e: 195935, 195949, 195950, 195954, 195955, 195958, 195989, 195994, 195998, 196000, 196006, 196085, 196089, 196090, 196091, 196099, 196105, 196107, 196185, 196187, 196194, 196197, 196199, 196200, 196201, 196203, 196208, 196214, 196308, 196309, 196310, 196311

- LHC13f: 196433, 196474, 196475, 196477, 196528, 196533, 196535, 196563, 196564, 196566, 196568, 196601, 196605, 196608, 196646, 196648, 196701, 196702, 196703, 196706, 196714, 196720, 196721, 196722, 196772, 196773, 196774, 196869, 196870, 196874, 196876, 196965, 196967, 196972, 196973, 196974, 197003, 197011, 197012, 197015, 197027, 197031, 197089, 197090, 197091, 197092, 197094, 197098, 197099, 197138, 197139, 197142, 197143, 197144, 197145, 197147, 197148, 197149, 197150, 197152, 197153, 197184, 197189, 197247, 197248, 197254, 197255, 197256, 197258, 197260, 197296, 197297, 197298, 197299, 197300, 197302, 197341, 197342, 197348, $197349,197351,197386,197387,197388$

- LHC16a1c: 183916, 184127, 184131, 184132, 184134, 184135, 184137, 184138, 184140, 184144, 184145, 184147, 184183, 184188, 184208, 184209, 184210, 184215, 184216, 184371, 184673, 184678, 184682, 184687, 184784, 184786, 184928, 184933, 184938, 184964, 184967, 184968, 184987, 184988, 184990, 185029, 185031, 185116, 185126, 185127, 185132, 185133, 185134, 185157, 185160, 185164, 185189, 185196, 185198, 185203, 185206, 185208, 185217, 185221, 185282, 185284, 185288, 185289, 185292, 185293, 185296, 185299, 185300, 185302, 185303, 185348, 185349, 185350, 185351, 185356, 185359, 185360, 185361, 185362, 185363, 185368, 185371, 185375, 185378, 185459, 185460, 185461, 185465, 185474, 185475, 185574, 185575, 185578, 185580, 185581, 185582, 185583, 185588, 185589, 185659, 185687, 185738, 185764, $185765,185768,185775,185776,185778,185784,185909,185915,186083,186163$, $186164,186165,186167,186205,186208,186319,186320$

- LHC1512b2: 176661, 176701, 176704, 176715, 176730, 176749, 176752, 176753, 176849, 176854,176859, 176924, 176926, 176927, 176929, 177011, 177157, 177160, 177167, 177173, 177182, 177597, 177601, 177612, 177620, 177624, 177671, 177798, 177799, 177804, 177858, 177860, 177861, 177869, 177938, 177942, 178163, 179569, 179571, 179584, 179585, 179591, 179618, 179621, 179639, 179796, 179802, 179803, 
179858, 179859, 179916, 179917, 179918, 179919, 179920, 180000, 180042, 180044, 180127, 180129, 180130, 180131, 180132, 180133, 180195, 180199, 180200, 180201, 180230, 180500, 180501, 180510, 180515, 180517, 180561, 180562, 180564, 180567, 180569, 180716, 180717, 180719, 180720, 182017, 182018, 182022, 182023, 182106, 182110, 182111, 182207, 182289, 182295, 182297, 182299, 182300, 182302, 182322, 182323, 182324, 182325, 182624, 182635, 182684, 182687, 182691, 182692, 182724, 182725, 182728, 182729, 182730, 182740, 182741, 182744, 183913, 183916, 184127, 184132, 184135, 184137, 184138, 184188, 184208, 184209, 184215, 184371, 184673, 184678, 184682, 184687, 184784, 184786, 185029, 185031, 185116, 185126, 185127, 185132, 185134, 185157, 185160, 185164, 185189, 185196, 185198, 185203, 185206, 185208, 185217, 185221, 185282, 185284, 185288, 185289, 185291, 185292, 185293, 185296, 185299, 185300, 185302, 185303, 185349, 185350, 185351, 185356, 185359, 185360, 185361, 185362, 185363, 185371, 185375, 185457, 185461, 185465, 185474, 185563, 185565, 185574, 185575, 185578, 185580, 185581, 185582, 185583, 185588, 185589, 185680, 185687, 185695, 185697, 185698, 185699, 185701, 185734, 185735, 185738, 185756, 185757, 185764, 185765, 185768, 185775, 185776, 185778, 185784, 185909, 185912, 186162, 186163, 186164, 186165, 186167, 186200, 186205, 186208, 186229,186318

- LHC14g3b: 195389, 195390, 195391, 195478, 195481, 195482, 195529, 195592, 195596, 195633, 195675

- LHC14b3a: 195724, 195727, 195760, 195765, 195767, 195783, 195787, 195826, 195827, 195830, 195831, 195867, 195871, 195872, 195873 


\section{A.3 Random Rejection of $[-10,-3] N_{\sigma T P C}^{e}$ Region}

The information about hadrons $\left(N_{\sigma T P C}^{e} \in[-10,-3]\right)$ is only used statistically (chapters 6.4 and 7.4 . Therefore, in order to save computing time, the tracks that have a $N_{\sigma T P C}^{e}$ in this region $\in[-10,-3]$ were rejected randomly in $80 \%$ of the cases. The $N_{\sigma T P C}^{e}$ region between the electron band $\left(N_{\sigma T P C}^{e} \in[-3,3]\right)$ and the one for hadrons was kept empty, so the statistical different regions were separated. Figure A.2 shows these regions and the corrected complete $N_{\sigma T P C}^{e}$ distribution, for pp. The random rejection procedure was performed in $\mathrm{pp}$ and $\mathrm{p}-\mathrm{Pb}$ data.

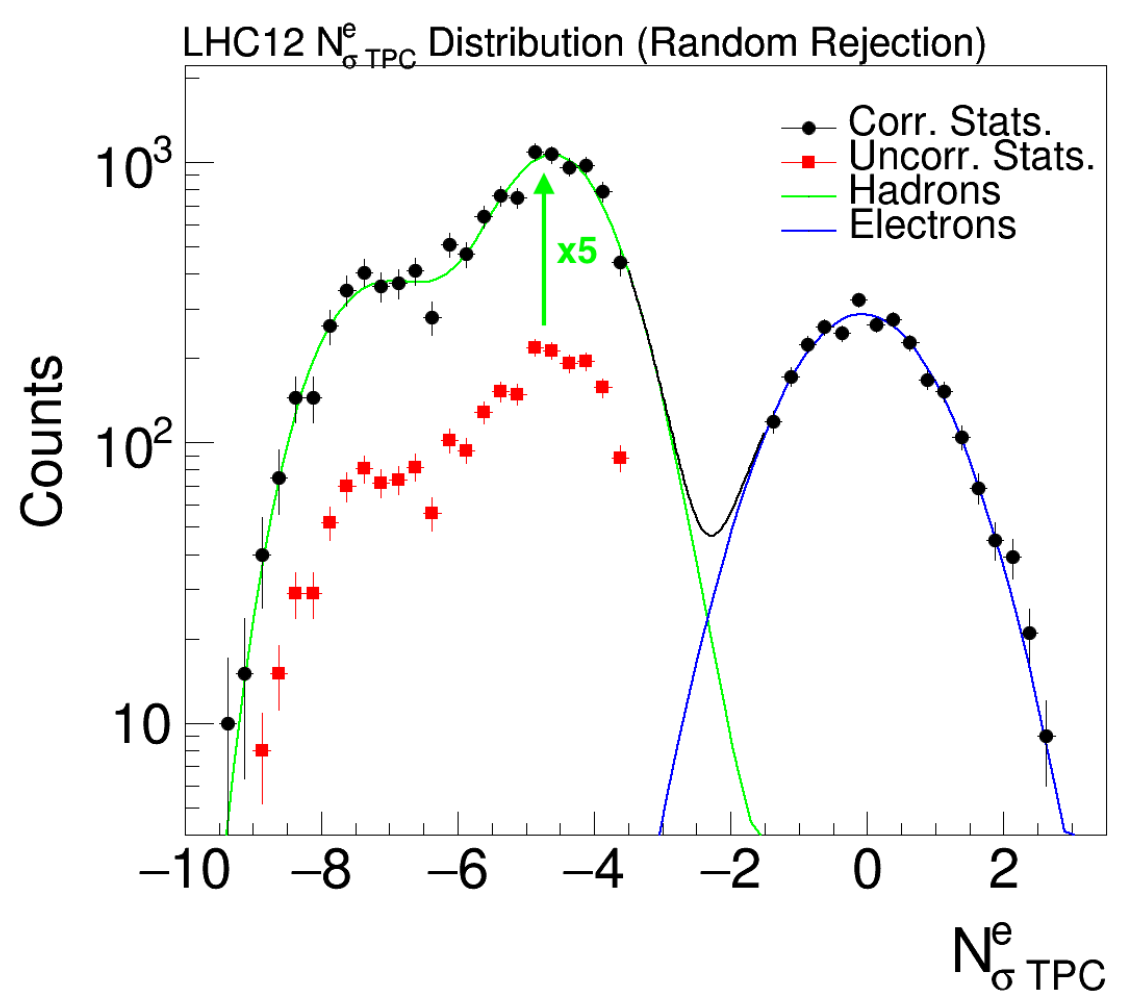

Figure A.2: $N_{\sigma T P C}^{e}$ random rejection in region $[-10,-3]$. The information in the region $\left.N_{\sigma T P C}^{e} \in\right]-3,-2\left[\right.$ is ignored, and the full data is recorded for $N_{\sigma T P C}^{e} \in[-2,3.5]$ (electron region). Black points are the corrected distributions; the red ones are the $20 \%$ that was accepted, randomly, in the region $N_{\sigma T P C}^{e} \in[-10,-3]$; and the green and blue lines are the fits for hadrons and electrons. The green arrow indicates the correction (multiplication by 5 ). 


\section{A.4 Middle Spectrum}

The inclusive electron selection for the e-jet pairs $J^{\text {incl. }}$ in the middle region $(\Delta \varphi \in$ $[\pi / 10, \pi / 2])$ follows the same procedure as in the others intervals. They are calculated in $p_{\mathrm{T}}^{e}$ ranges.

The hadronic contribution $J^{\text {had. }}$ was estimated and calculated by using the $E / p$ distribution and the $N_{\sigma T P C}^{e}$ for hadrons were selected in th range $[-10,-3]$. The hadronic $E / p$ curve was normalized (the normalization contant $A_{\text {norm. }}$ ) by using the range $E / p \in$ $[0.24,0.74]$, and the hadronic fraction $f_{h}$ was calcualted in the region $E / p \in[0.8,1.3]$.

The non heavy-flavour electron contribution $J^{c o n v}$, was estimated by the invariant mass technique (the figure A.3 shows the invariant mass distribution for pp and $\mathrm{p}-\mathrm{Pb}$ ). The unlike-sign and like-sign distributions were subtracted from one another and the efficiency was taken into account.

LHC12 - Invariant Mass Distribution

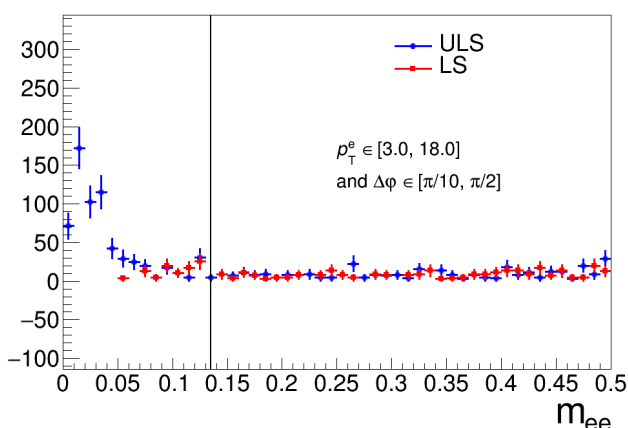

LHC13 - Invariant Mass Distribution

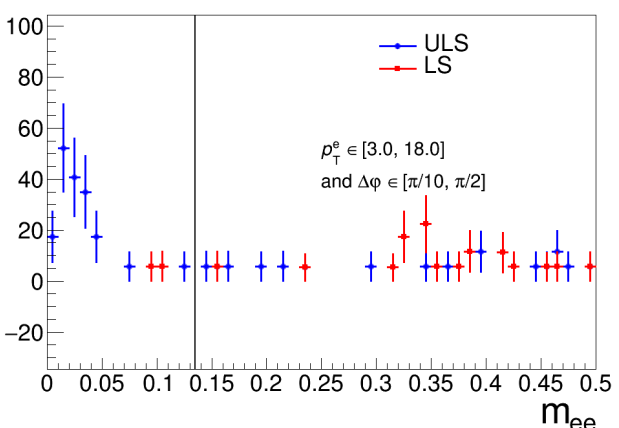

Figure A.3: Middle Invariant mass distribution for like-sign $e^{+} e^{-}$pairs (in red) and for unlikesign pairs (in blue). The black bar shows the mass cut $0.135 \mathrm{GeV}$ : only the region to its left is used in the conversion contribution. The data are from pp collisions are in the left-hand side and the $\mathrm{p}-\mathrm{Pb}$ data are in the right-hand side.

The normalization of the $p_{\mathrm{T}, \text { chjet }}$ and the $\Delta \varphi$ distributions were taken as the number of heavy-flavour decay electrons $N_{H F e}$.

All the calculated contributions - namely inclusive, hadronic and non-HFe - are shown in figures $\mathrm{A} .4$ (pp) and $\mathrm{A} .5$ (p-Pb) (left panel). They are already corrected by the efficiencies and normalized. The final, corrected and unfolded spectrum are shown in figures $\mathrm{A} .4$ (pp) and A.5 (p-Pb), right panel. 

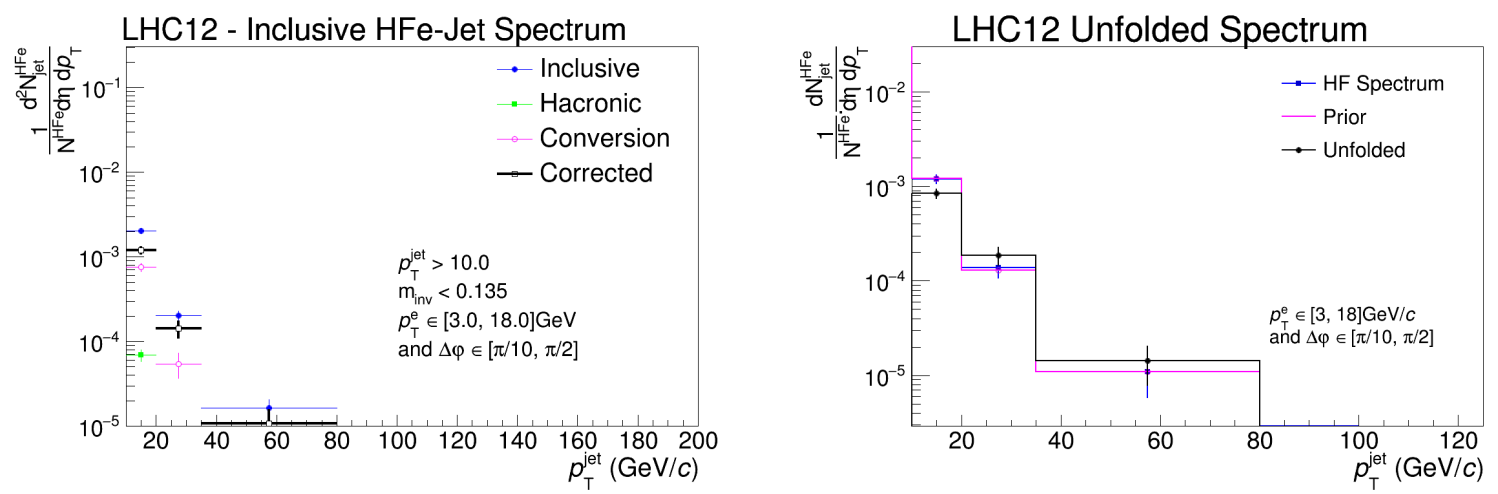

Figure A.4: Middle: In the left panel, the contributions for the e-jet distribution are shown, namely inclusive (blue), hadronic (green) and non-HFe (magenta). The corrected one is in black. In the right panel, the unfolded spectrum is shown. The prior used in the unfolding procedure is in magenta. Data from pp collisions.
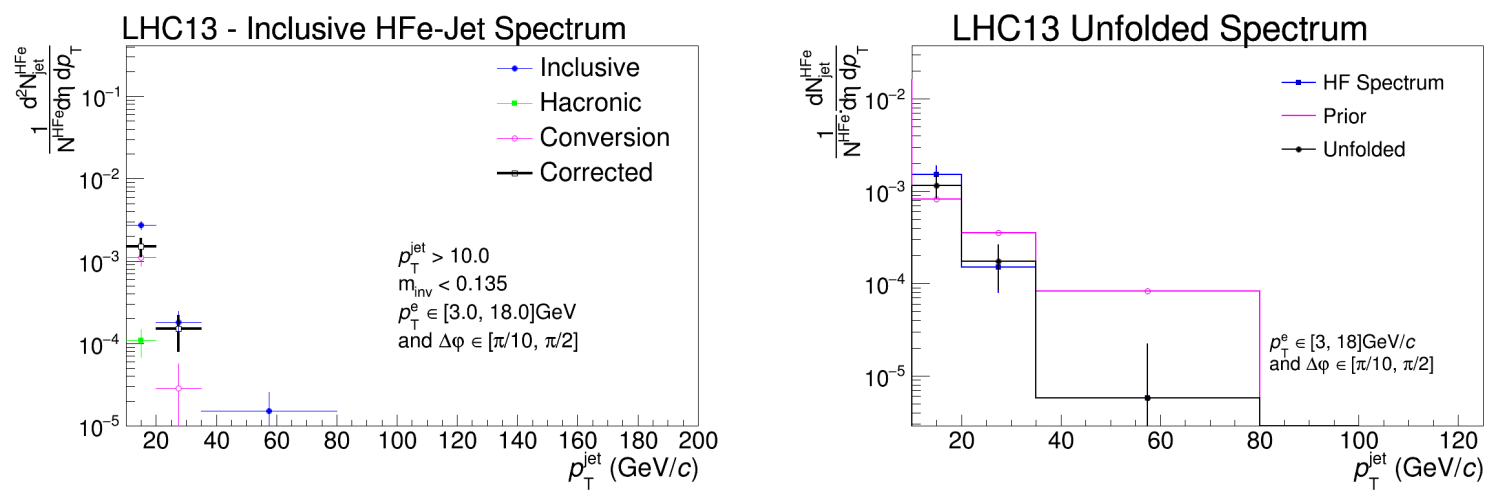

Figure A.5: Middle: In the left panel, the contributions for the e-jet distribution are shown, namely inclusive (blue), hadronic (green) and non-HFe (magenta). The corrected one is in black. In the right panel, the unfolded spectrum is shown. The prior used in the unfolding procedure is in magenta. Data from $\mathrm{p}-\mathrm{Pb}$ collisions. 


\section{A.5 Systematic Uncertainties for $\Delta_{\text {side }}$}

The tables A.1 and A.2 show the systematic uncertainties for the observable $\Delta_{\text {side }}$, that was presented in section 8.2 . 
Table A.1: Variation of cuts and selections for systematic uncertainty calculation in pp for the $\Delta_{\text {side }}$.

\begin{tabular}{|c|c|c|c|c|}
\hline Variable & Variation(s) & Standard & $\begin{array}{c}\text { Syst. Uncert. } \\
\text { Away side }\end{array}$ & $\begin{array}{l}\text { Syst. Uncert } \\
\text { Near side }\end{array}$ \\
\hline Minimum TPC Clusters & 90,110 & 80 & & \\
\hline Min. TPC Clusters (PID) & 90,110 & 80 & $<2 \%$ & $<2 \%$ \\
\hline Min. Hits in ITS & 3,4 & $-{ }^{-}$ & $<\overline{2}$ & $<10$ \\
\hline${ }^{-\cdots} \overline{\text { Minimum }} \bar{N}_{\sigma T P C}^{e^{-}--}$ & $-1.0,-0.5,0.0,0 . \overline{2} 5,0.5$ & -1.5 & $-28 \%$ & $<110$ \\
\hline Maximum $N_{\sigma \underline{T}}^{e} P_{-} C_{-}$ & $2.5,3.5$ & 3.0 & $<28 \%$ & $<11 \%$ \\
\hline Minimum $\bar{E} / p$ & $0 . \overline{7} 6, \overline{0} \overline{7} \overline{8}, 0 . \overline{8} 2,0 . \overline{8}, 0 . \overline{8} 6$ & $0 . \overline{8} 0^{-}$ & $<4 \%$ & $<4 \%$ \\
\hline Maximum $E / p$ & 1.2 & 1.3 & $<4 \%$ & $<4 \%$ \\
\hline Maximum $\overline{M 20}$ & $0.2,0.4$ & $0 . \overline{3}$ & $<30 \%$ & $<16 \%$ \\
\hline Maximum $\left|\bar{\Delta} \varphi_{\text {match }}\right|$ & 0.03 & 0.05 & $\approx 0 \%$ & $<\overline{4} \%$ \\
\hline Min. $\overline{\mathrm{T}} \overline{\mathrm{C}}$ Clusters (Partner) & $-80^{---}$ & $--\overline{70}--$ & $\approx \overline{0} \%$ & $<\overline{1} \%$ \\
\hline Minimum $p_{\mathrm{T}_{-}, \bar{p} a \bar{r} t \bar{n} e \bar{r}}$ & $1.0 \overline{\mathrm{Ge}} \overline{\mathrm{e}} / c^{-\cdots}$ & $0.15 \mathrm{GeV} / c$ & $\approx 0 \%$ & $<31 \%$ \\
\hline Maximum $m_{\text {inv. }}$ & $0.100 \mathrm{GeV}, 0.180 \overline{G e V}^{-}$ & $0.135 \mathrm{GeV}$ & $\approx 0 \%$ & $<\overline{5} \%$ \\
\hline Maximum $\left|\bar{N}_{\sigma T P C}^{e^{-}}\right| \overline{\text { Partner }}$ & $\overline{2} . \overline{5}-\cdots-$ & $-\overline{3} .0^{----}$ & $-\overline{4} 0 \overline{0}$ & $-\overline{1} \bar{\gamma}$ \\
\hline Unfolding Method & $\overline{S V D}$ & Iterative Bayesian & & \\
\hline Unfolding Parameter $K$ & $K+1$ & $K=3$ & $<17 \%$ & $<24 \%$ \\
\hline Unfolding Prior & Power Law & Response Y-Projection & & \\
\hline
\end{tabular}


Table A.2: Variation of cuts and selections for systematic uncertainty calculation in $\mathrm{p}-\mathrm{Pb}$ for the $\Delta_{\text {side }}$.

\begin{tabular}{|c|c|c|c|c|}
\hline Variable & Variation(s) & Standard & $\begin{array}{l}\text { Syst. Uncert. } \\
\text { Away side }\end{array}$ & $\begin{array}{l}\text { Syst. Uncert. } \\
\text { Near side }\end{array}$ \\
\hline Minimum TPC Clusters & 90,110 & 80 & & \\
\hline Min. TPC Clusters (PID) & 90,110 & 80 & & $\approx 0 \%$ \\
\hline Min. Hits in $\overline{I T S}^{-}$ & 3,4 & $-\overline{2}$ & $\approx \overline{0} \%$ & $<56 \%$ \\
\hline Minimum $\bar{N}_{\sigma T P C}^{e}$ & $-1.0,-0.5,0.0,0 . \overline{2} 5,0.5$ & -1.5 & $<348 \%$ & $<53 \%$ \\
\hline Maximum $N_{\sigma T}^{e} P_{-} C_{-} \ldots$ & $\begin{array}{c}2.5,3.5 \\
-\end{array}$ & 3.0 & & ------ \\
\hline Minimum $E / p$ & $0.76,0.78,0.82,0.84,0.86$ & $\begin{array}{c}0.80 \\
13\end{array}$ & $<10 \%$ & $<9 \%$ \\
\hline 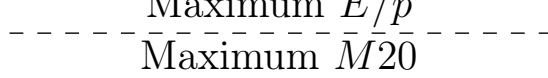 & $------\frac{1.2}{0.2,}, 0.4^{---}$ & $------\frac{1.3}{0 . \overline{3}}$ & $--\overline{<} \overline{17} \overline{\%}$ & $--\overline{41} \overline{\%}$ \\
\hline${ }^{--} \overline{\text { Maximum }}\left|\bar{\Delta} \bar{\varphi}_{\text {match }}\right|-{ }^{-}$ & 0.03 & $0 . \overline{0} 5$ & $\approx \overline{0} \overline{\%}$ & $\approx \overline{0} \%$ \\
\hline 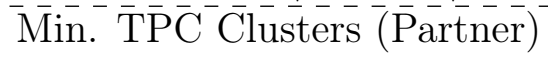 & $8 \overline{0}$ & 70 & $\approx 0 \%$ & $\approx 0 \%$ \\
\hline Minimum $p_{\mathrm{T}}^{\bar{e}, \bar{p} a \bar{r} t \bar{n} e \bar{r}}$ & $1.0 \overline{\mathrm{GeV}} / c$ & $0.15 \mathrm{GeV} / \bar{c}$ & $\approx \overline{0} \%$ & $<14 \%$ \\
\hline${ }^{-} \overline{\text { Maximum } m_{i n v}}$ & $\overline{0} . \overline{100} \overline{\mathrm{G}} \overline{\mathrm{eV}} \overline{0}, \overline{180} \overline{\mathrm{GeV}}$ & $-\overline{0.135 \overline{\mathrm{G}} \mathrm{eV}}$ & $-\overline{6} \overline{0} \%$ & $\overline{<} \overline{12} \overline{\%} \%$ \\
\hline Maximum $\left|N_{\sigma T P C}^{e}\right|{ }_{-}^{e}$ (Partner & $--\overline{2} . \overline{5}--$ & $\begin{array}{c}--1.0---1 \\
-\end{array}$ & $<\overline{43} \overline{\%}$ & $\approx \overline{0} \%$ \\
\hline Ūnfolding Methò $\overline{\text { Met }}{ }^{---}$ & $\overline{\mathrm{SVD}}$ & Iterative Bayesian & & \\
\hline Unfolding Parameter $K$ & $K+1$ & $K=3$ & $<151 \%$ & $<20 \%$ \\
\hline Unfolding Prior & Power Law & Response Y-Projection & & \\
\hline
\end{tabular}




\section{A.6 Closure Test}

In order to test the analysis chain and methods, the Monte Carlo simulations LHC16a1c (pp) and LHC14g3b (p-Pb) were used. The standard and complete analysis were performed on them so equivalent results to the final spectra in sections 6.10 and 7.10 were obtained.

In addition, the generator information could be accessed and the true observable was also obtained: the true heavy-flavour decay electron were paired to jets and their $p_{\mathrm{T}, \mathrm{ch}}$ jet distribution could be compared to the one from the reconstruction data. They are shown in figures A.6 and A.7, for pp and p-Pb respectively, for the away side. The near side observables are in figures A.8 and A.9. In all cases, the results from the generator (true distribution) and the one from the analysis methods are compatible within uncertainties, but the low $p_{\mathrm{T}}$ region has larger uncertainties due to the low-momentum background that is not perfectly simulated.

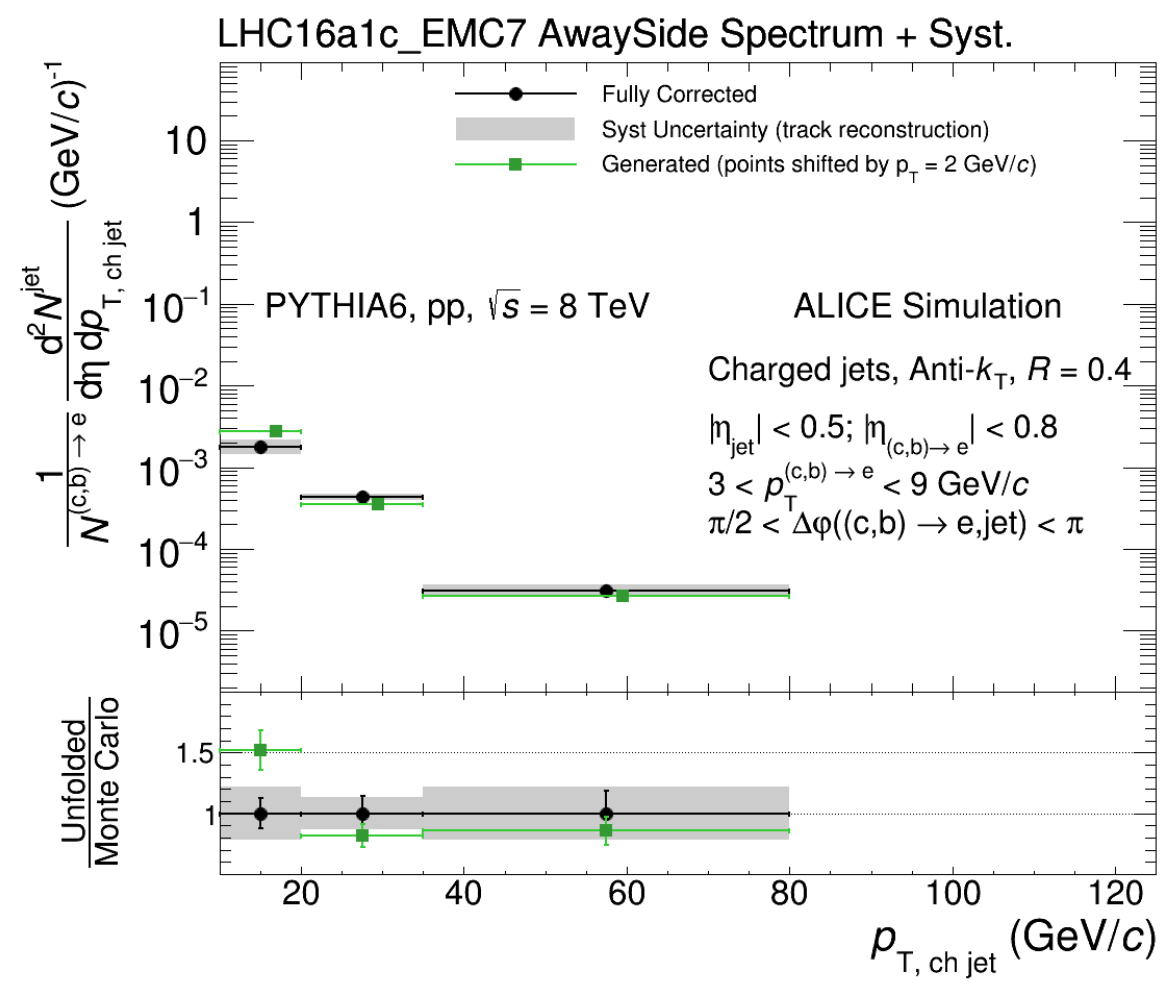

Figure A.6: Away side Closure test spectrum for pp. The black points are the measured spetrum and the green ones are the true Monte Carlo spectrum (top panel). Bottom panel shows their ratio. 


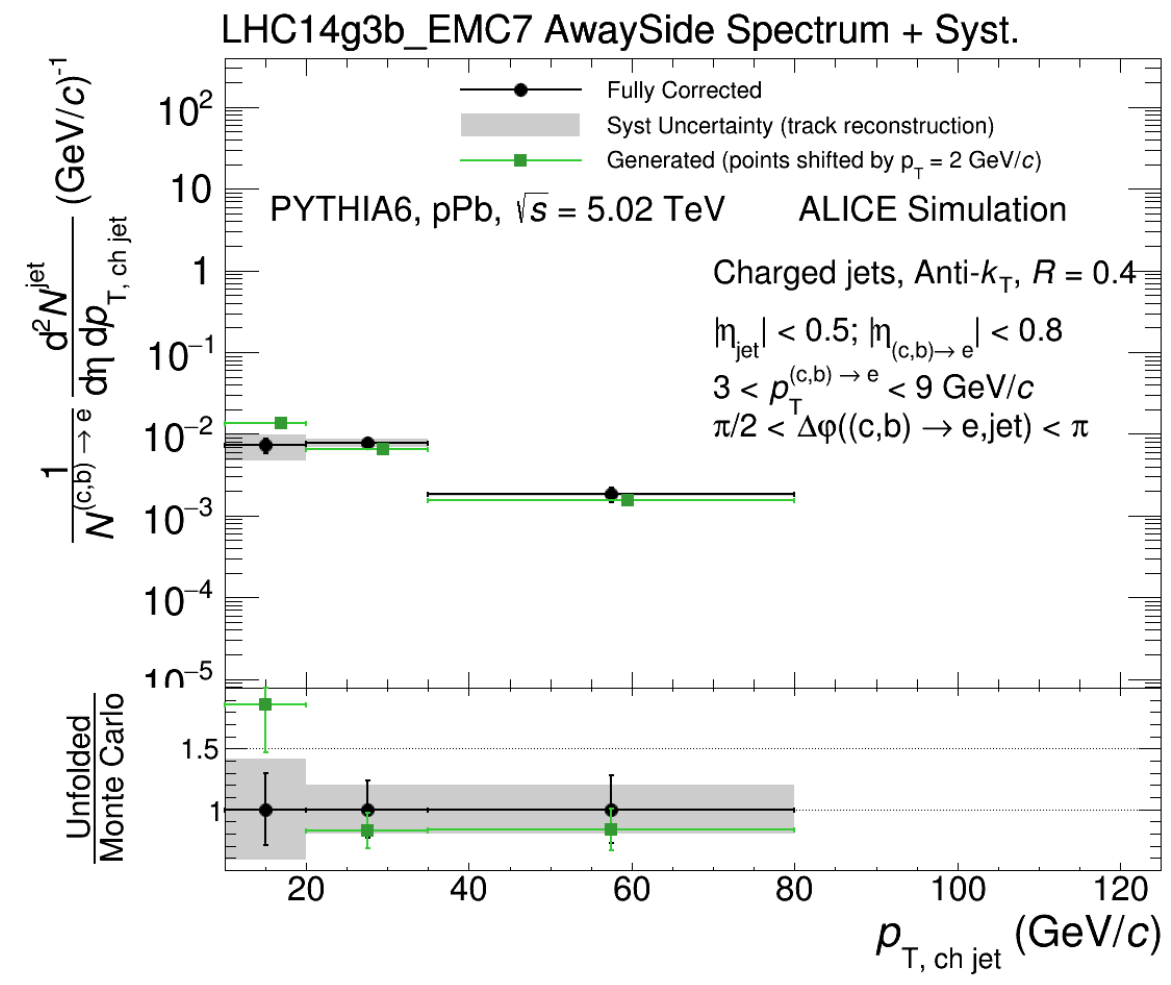

Figure A.7: Away side Closure test spectrum for p-Pb. The black points are the measured spetrum and the green ones are the true Monte Carlo spectrum (top panel). Bottom panel shows their ratio.

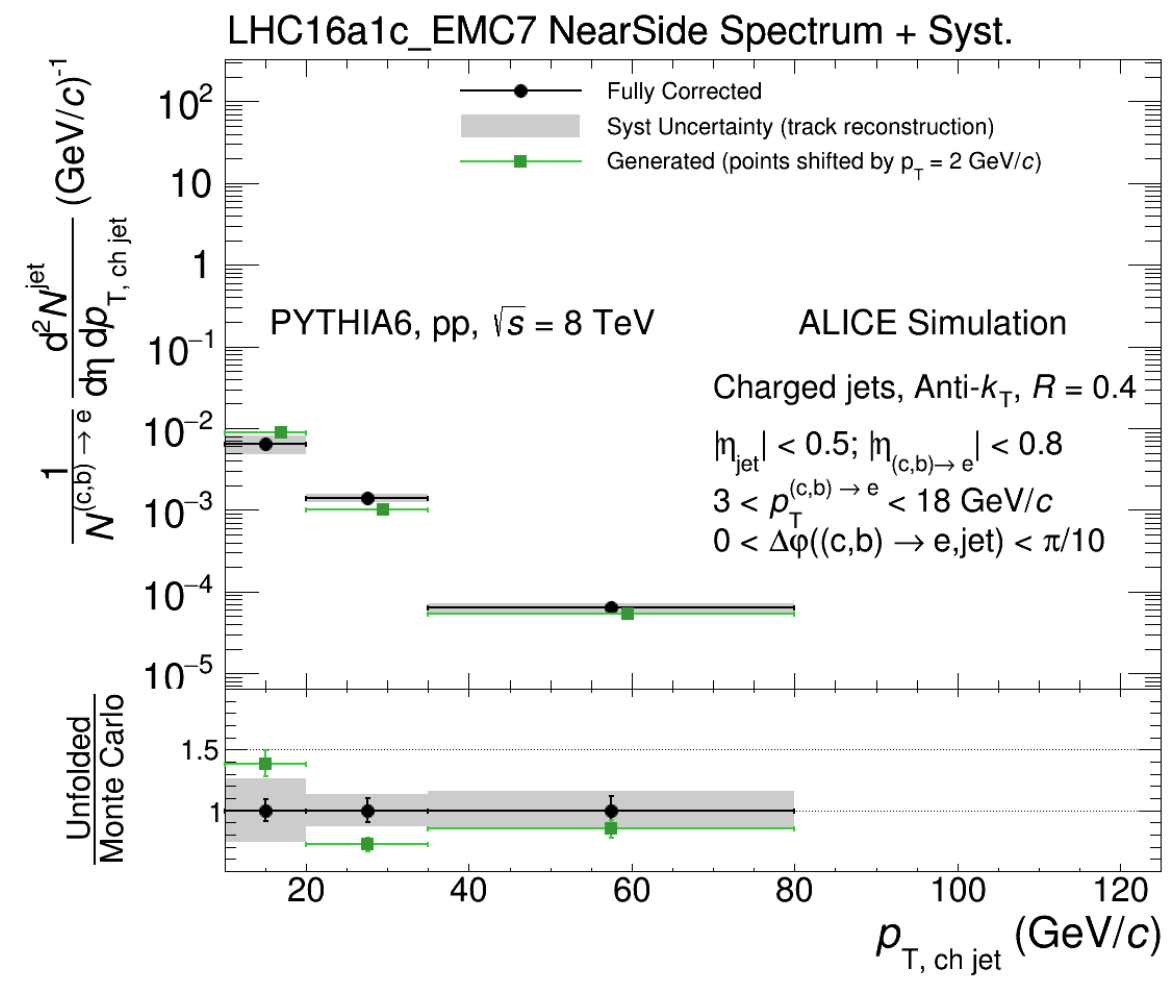

Figure A.8: Near side Closure test spectrum for pp. The black points are the measured spetrum and the green ones are the true Monte Carlo spectrum (top panel). Bottom panel shows their ratio. 


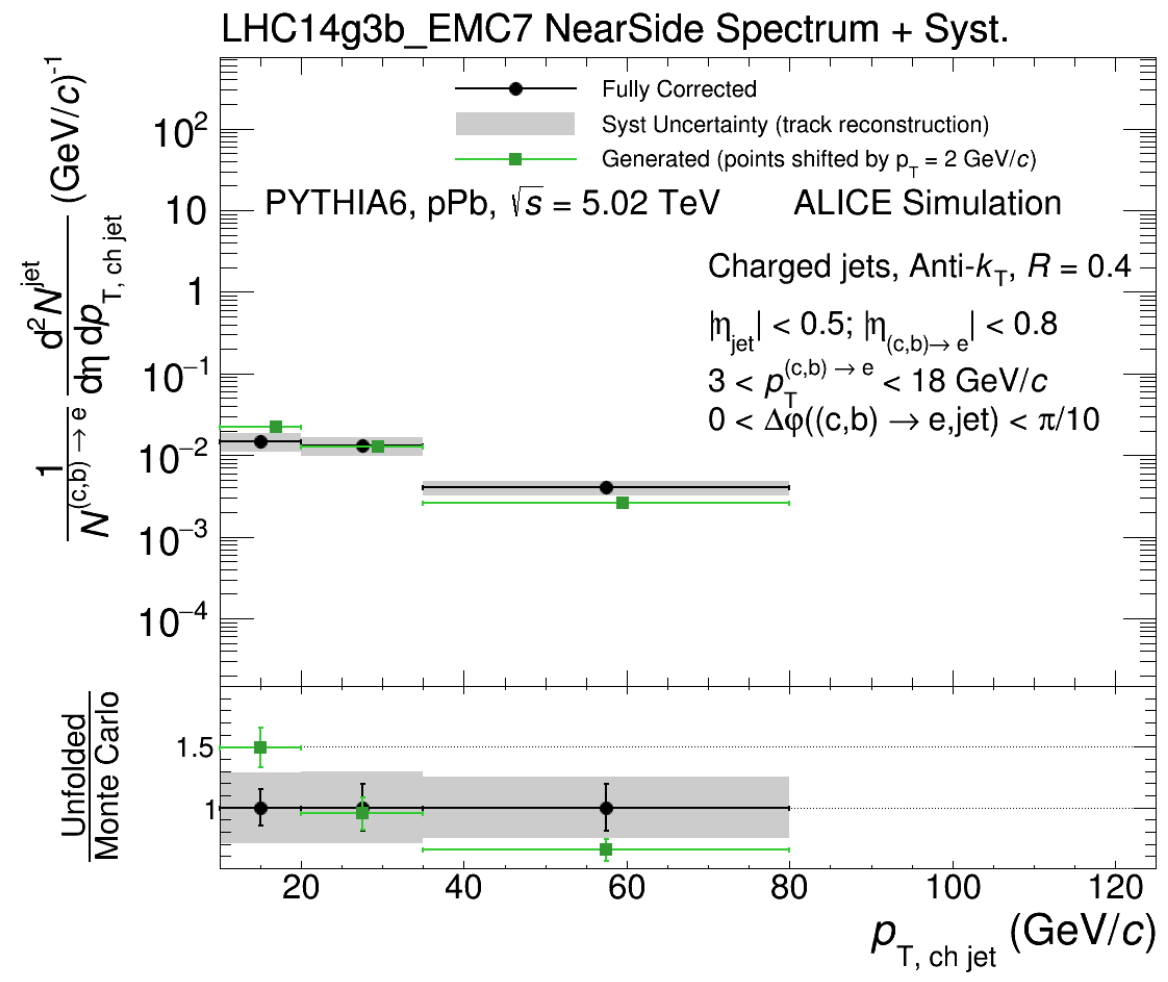

Figure A.9: Near side Closure test spectrum for $\mathrm{p}-\mathrm{Pb}$. The black points are the measured spetrum and the green ones are the true Monte Carlo spectrum (top panel). Bottom panel shows their ratio. 


\section{A.7 Unfolding Uncertainties}

The unfolding procedure could increase the uncertainties of the spectrum that is not reflected in its output uncertainty values. In order to verify this, one can compare how a variation in the measured input spectrum affects the unfolded output one.

The original measured spectrum $H_{0}$ bin contents were smeared with a gaussian, whose deviation was the uncertainty of the bin. This resulted in a smeared spectrum $H_{1}$, that unfolds into $\mathcal{H}_{1}$. Its difference with respect to the original unfolded spectrum $\mathcal{H}_{0}$ was calculated in therms of the original unfolded uncertainty $\sigma_{0, b i n}$ (equation A.4), for each bin.

$$
\Delta \mathcal{H}_{i, b i n}=\frac{\left|\mathcal{H}_{i, b i n}-\mathcal{H}_{0, b i n}\right|}{\sigma_{0, b i n}}
$$

The distribution of these variations $\Delta \mathcal{H}$ are shown in figure A.10 and A.11, for pp and $\mathrm{p}-\mathrm{Pb}$ respectively. For each bin, i.e. $p_{\mathrm{T}, \text { chjet }}$ interval, a gaussian was fit to the distribution, whose deviations are shown in figure A.10 and is around the unity. This means that, for variations that are compatible with the measured uncertainties, their unfolded spectra are compatible within the unfolded uncertainty, so there is no uncertainty that was not taken into account to be added.

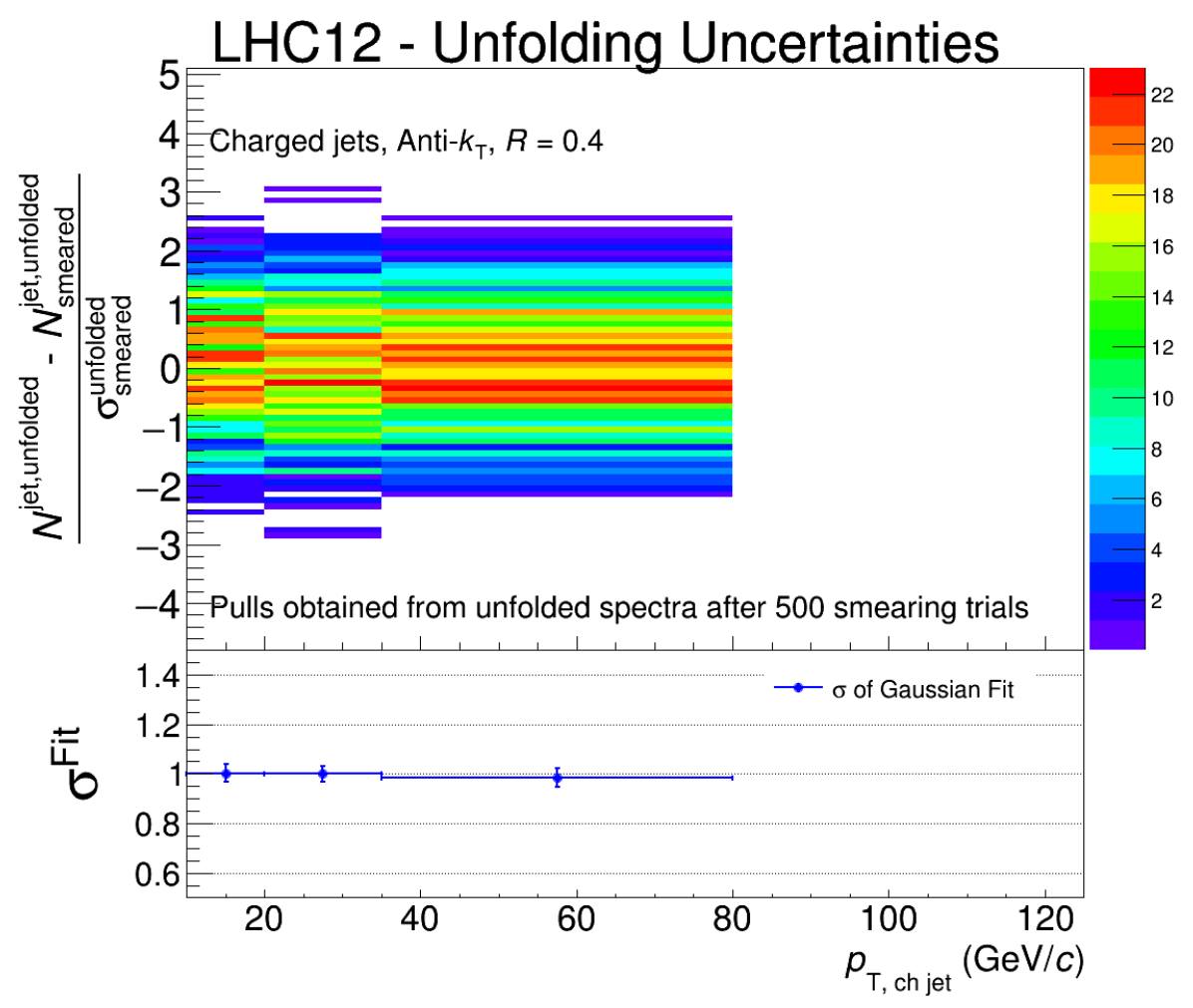

Figure A.10: Unfolding $\Delta \mathcal{H}$ distribution for the away side spectrum (top). The deviation from a Gaussian fit to each $p_{\text {T,chjet }}$ bin distribution is shown in the bottom panel. Data from pp collisions data. 


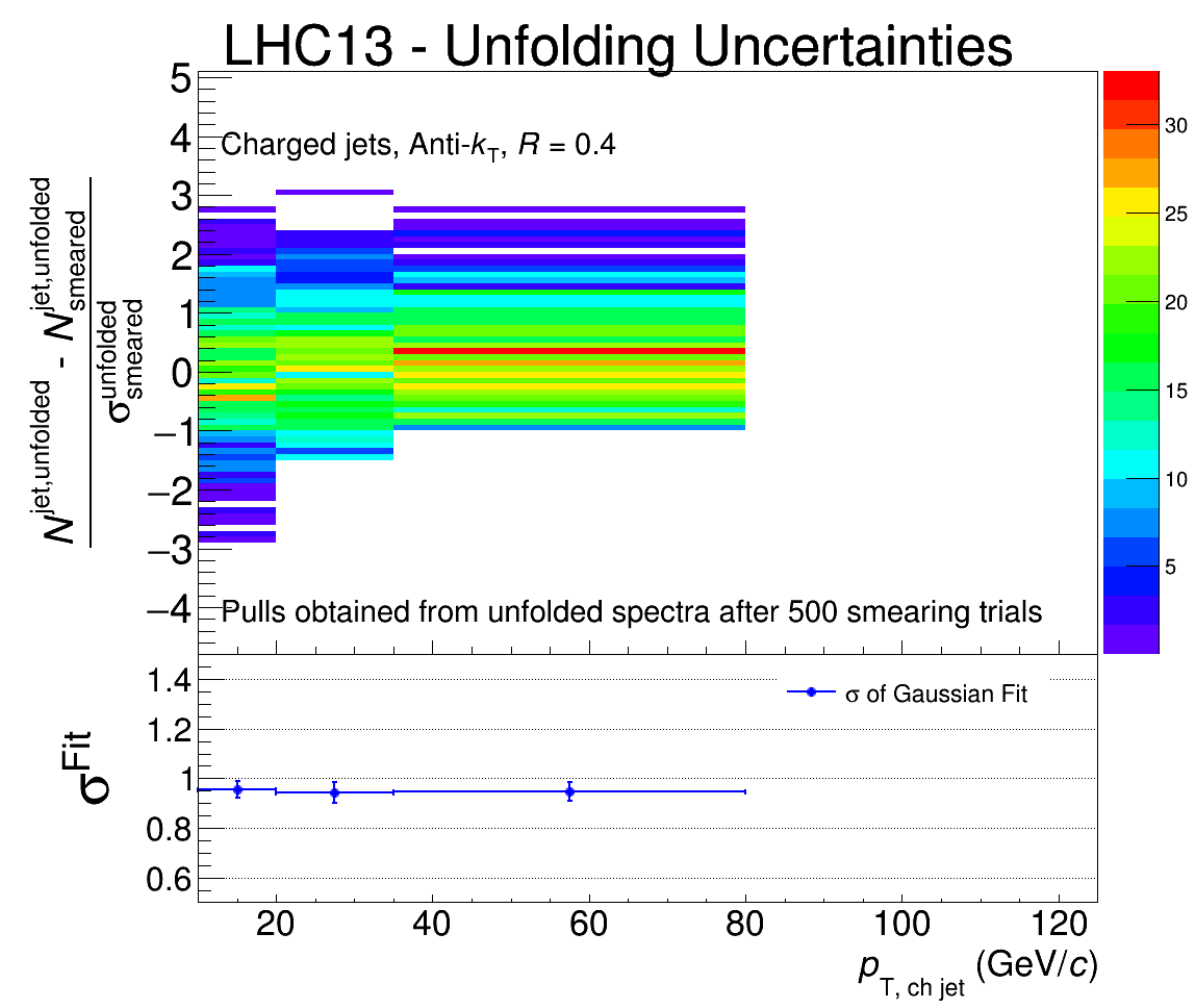

Figure A.11: Unfolding $\Delta \mathcal{H}$ distribution for the away side spectrum (top). The deviation from a Gaussian fit to each $p_{\mathrm{T} \text {,chjet }}$ bin distribution is shown in the bottom panel. Data from $\mathrm{p}-\mathrm{Pb}$ collisions data. 


\section{A.8 FONLL Scaling Factor}

The cross sections of charm and bottom decay electrons were calculated with the FONLL framework [63, 64], for the two centre of mass energies $\sqrt{s}$, namely $8 \mathrm{TeV}$ and $5.02 \mathrm{TeV}$ (figure A.12).

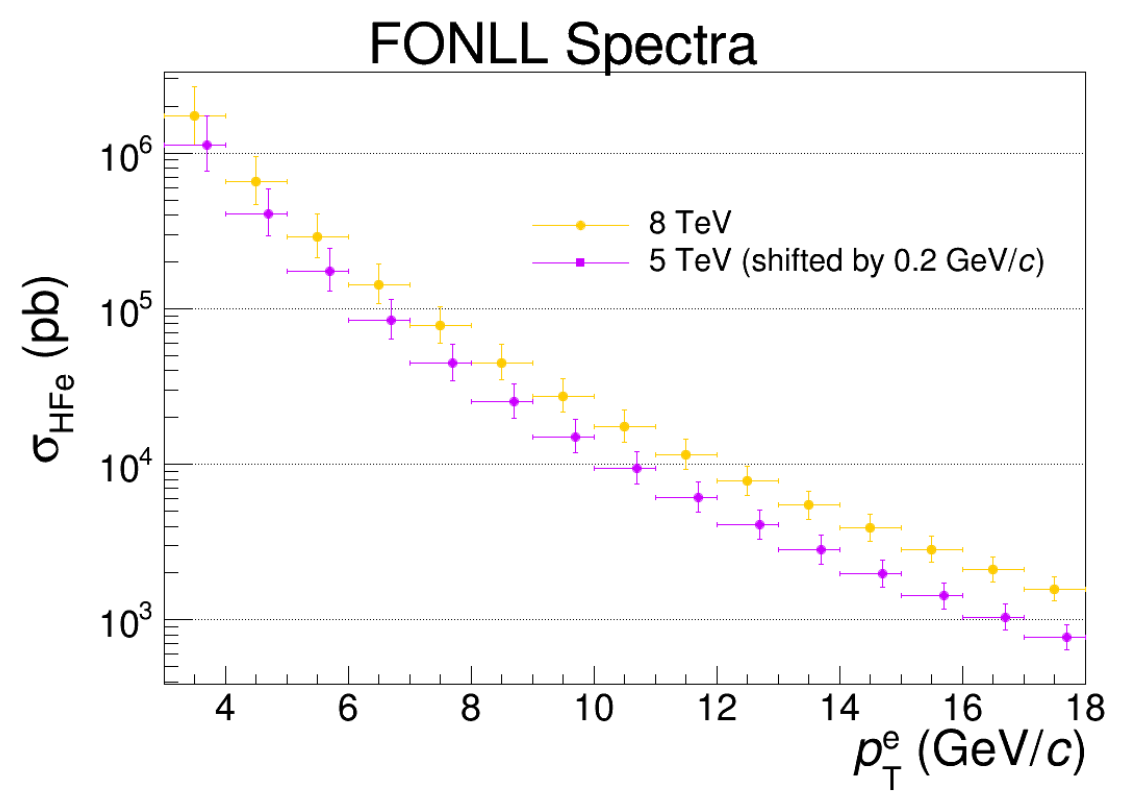

Figure A.12: FONLL cross section [63, 64] for heavy-flavour decay electrons at $5.02 \mathrm{TeV}$ (in violet) and $8 \mathrm{TeV}$ (in orange).

The ratio $f_{8 \rightarrow 5}$ of their $p_{\mathrm{T}}$ spectra was taken as the scale factor between the different energies (shown in figure A.13). The values of the cross section at the edges of the asymmetric errors were used to calculate a maximum $\sigma_{H F e}^{\max }$ and a minimum $\sigma_{H F e}^{\min }$ spectra, for each energy. These spectra were used to calculate a maximum $f_{8 \rightarrow 5}^{\max }=\frac{\sigma_{\max }}{\sigma_{\min }}$ and a minimum $f_{8 \rightarrow 5}^{\min }=\frac{\sigma_{H F e}^{m i n}}{\sigma_{H F e}^{m a x}}$ ratio spectra, whose values were taken as the final FONLL uncertainties edges.

For a given electron $p_{\mathrm{T}}^{e}$ range, the corresponding jet $p_{\mathrm{T} \text {,chjet }}$ spectra was scaled. This was done for all the $p_{\mathrm{T}}^{e}$ ranges, and the resulting jet $p_{\mathrm{T}, \text { chjet }}$ spectra were summed. The normalization, that is simply the number of electrons, was scaled also according to these FONLL correction values. When both are combined, i.e. the scaled spectra is normalized by the scaled normalization, the result is almost the same compared to the unscaled observable, which means that this scaling introduces more uncertainties without changing the final result.

The scaling factor uncertainties were propagated to the observable (figures A.14 and A.15 and the results show that the statistical uncertainty is greater or of the same order of the FONLL one. In addition, the calculated total systematic uncertainties (chapter 6.10) are much greater than the statistical ones. 


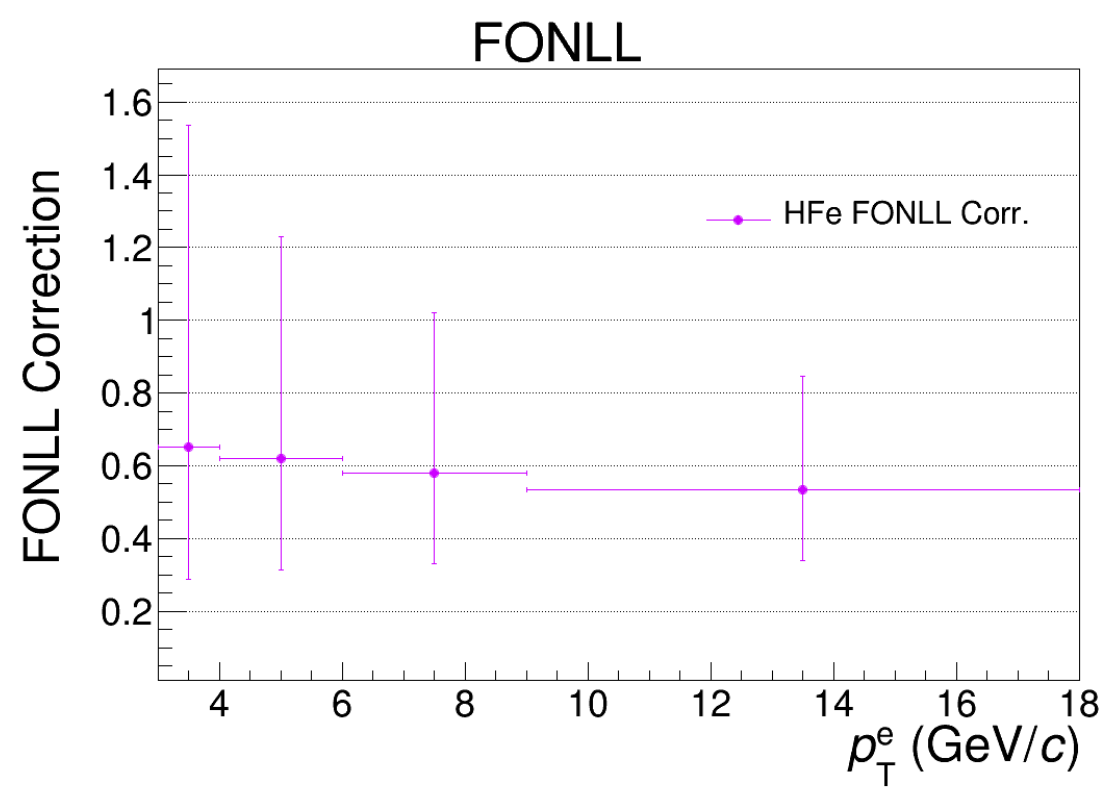

Figure A.13: FONLL [63, 64] energy scale factor is the ratio between the heavy-flavour decay electrons cross section in pp collisions at $5.02 \mathrm{TeV}$ and $8 \mathrm{TeV}$.

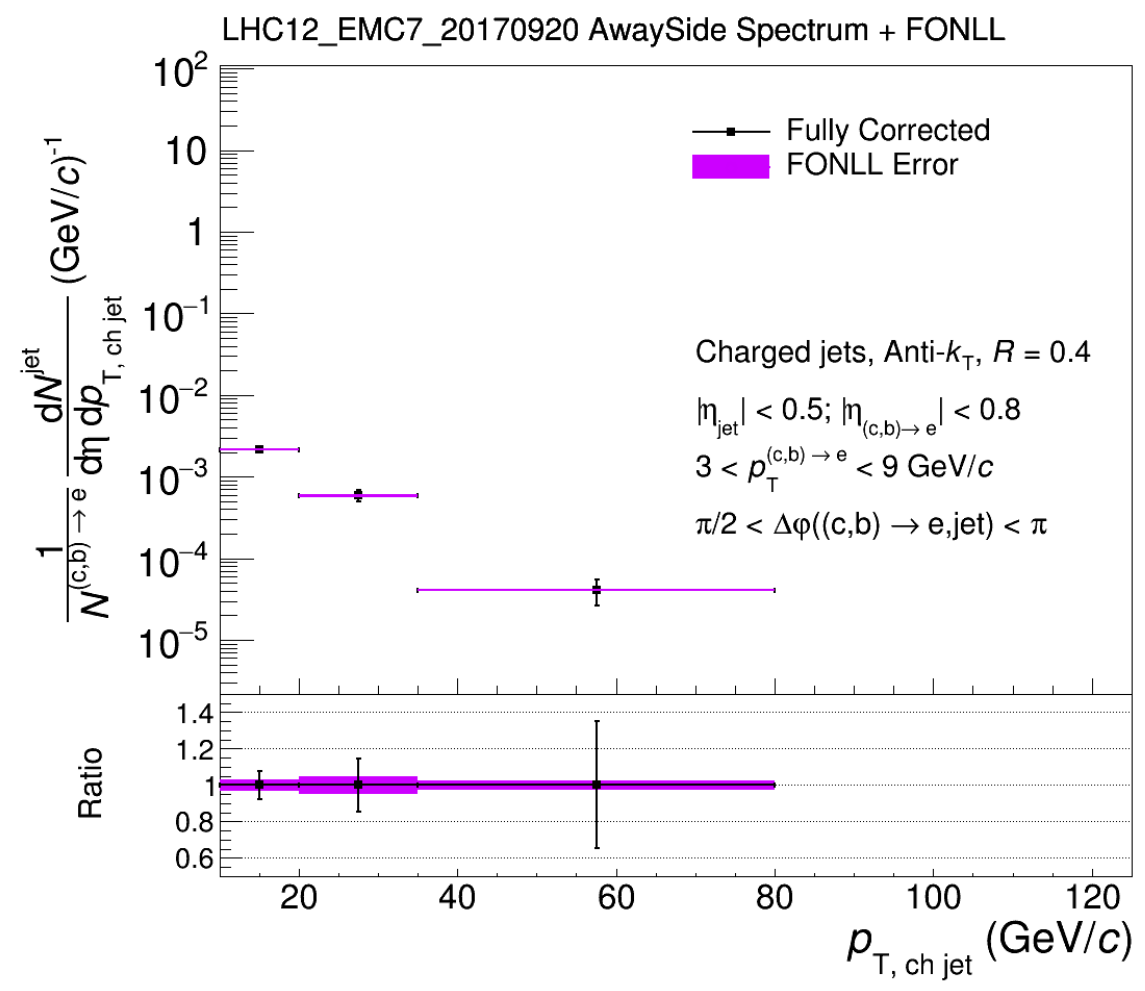

Figure A.14: Away side: HFe-jet spectrum, after the energy scaling, with its FONLL uncertainties (top panel). The bottom panel shows the ratio between the spectra with statistical uncertainties only, and with the FONLL uncertainties only. 


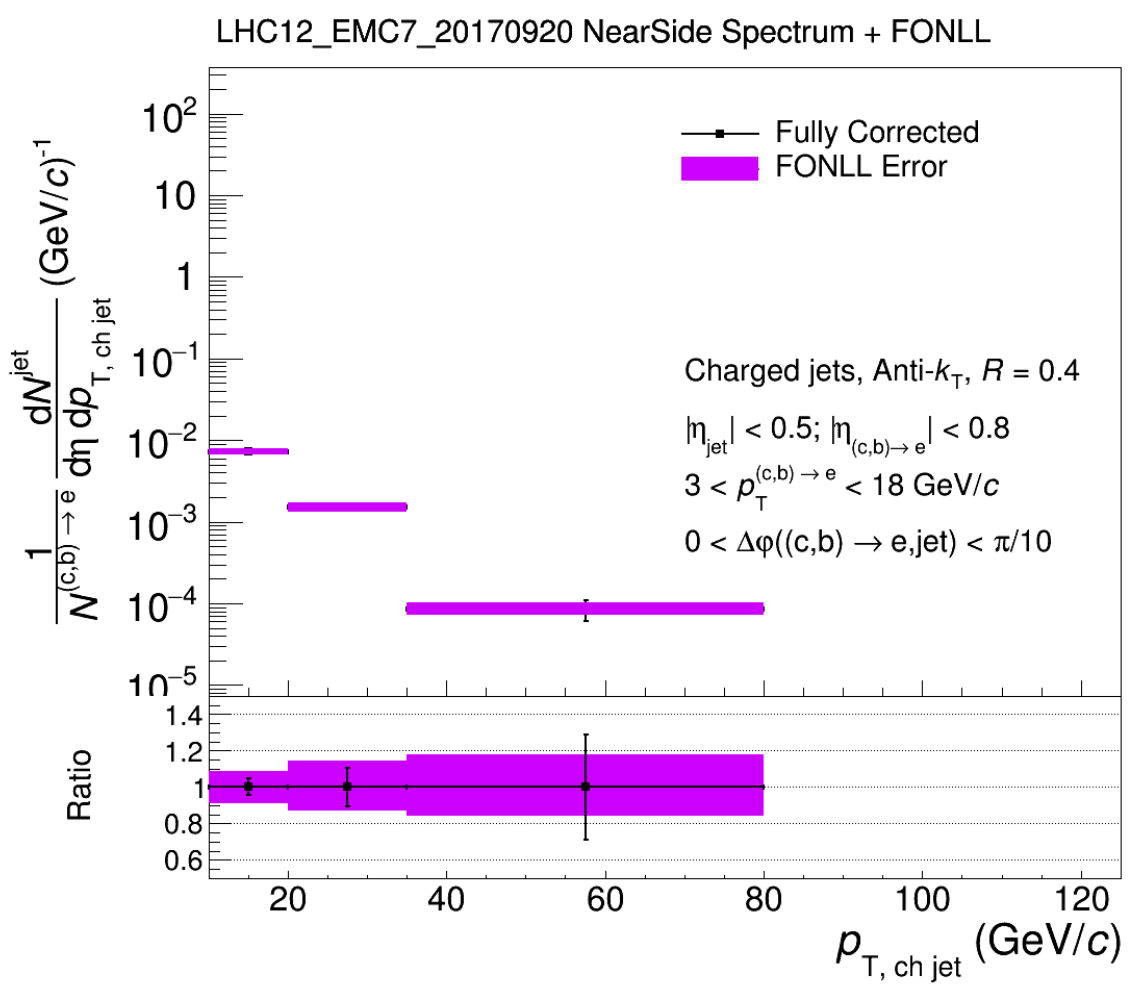

Figure A.15: Near side: HFe-jet spectrum, after the energy scaling, with its FONLL uncertainties (top panel). The bottom panel shows the ratio between the spectra with statistical uncertainties only, and with the FONLL uncertainties only. 


\section{Glossary}

EMC7 triggered event class that requires an EMCal minimum signal. 59, 69, 85

$R_{A A}$ Nuclear Modification Factor for nucleus-nucleus collisions. 26

$R_{A B}$ Nuclear Modification Factor for A-B systems collisions. 26, 29, 37

$\eta$ Pseudorapidity, a geometrical coordinate. 70, 105

$\nu_{2}$ Eliptic Azimutal Anisotropy. 28

$p_{\mathrm{T}}$ Transverse momentum. 105

y Rapidity, a relativistic quantity related to the logitudinal-transverse energy ratio. 105

ALICE A Large Ion Collider Experiment. 49

CERN European Organization for Nuclear Research. 49, 50

CNM Cold Nuclear Matter. 15, 27, 97

collinear safety a characteristic of a jet clusterization scheme that does not change the resulting jets when a particle is substituted by two others, with the same direction the former (collinear) and same (transverse) momentum sum. 40

EMCal Electromagnetic Calorimeter. 16, 49

event Collision. 69, 85

Fastjet Package of libraries that implement several jet reconstruction algorithms. 64

heavy-flavour Related to quarks whose masses are much larger than the ordinary ones', namely charm, bottom and top. 15, 33

infrared safety a characteristic of a jet clusterization scheme that does not change the resulting jets when a low (transverse) momentum particle is added to the set. 40 
invariant mass Invariant mass, the four-momentum contraction $p_{\mu} p^{\mu}$, that is invariant through frame of reference transformation. $67,74,89,111$

ITS Inner Tracking System. 49

jet Collimated spray of particles. Algorithm dependent. 38

LHC Large Hadron Colider. 15, 49, 50

IQCD Lattice Quantum Chromodynamics. 23

PHENIX Pioneering High Energy Nuclear Interaction Experiment. 28

pQCD Perturbative Quantum Chromodynamics. 15

primary vertex the position of the collision point reconstructed by propagating the tracks and combining their closest approach point. 51, 69, 85

QCD Quantum Chromodynamics. 15

QGP Quark Gluon Plasma. 15, 21

RHIC Relativistic Heavy-Ion Collider. 15

Standard Model The ensemble of QED, Electro Weak theory and QCD. 17

STAR Solenoidal Tracker At RHIC. 28

TPC Time Projection Chamber. 16, 49

unfolding Process that tries to recover the true, original distribution from a measured one. $43,77,91$ 


\section{Bibliography}

[1] H. Satz, "The Thermodynamics of Quarks and Gluons," Lect. Notes Phys., vol. 785, pp. 1-21, 2010, 0803.1611.

[2] S. Bethke, "Experimental tests of asymptotic freedom," Progress in Particle and Nuclear Physics, vol. 58, no. 2, pp. 351 - 386, 2007.

[3] J. Adams, M. Aggarwal, Z. Ahammed, et al., "Experimental and theoretical challenges in the search for the quark-gluon plasma: The STAR collaboration's critical assessment of the evidence from RHIC collisions," Nuclear Physics A, vol. 757, no. 1-2, pp. $102-183$, 2005. First Three Years of Operation of RHIC.

[4] K. Adcox, S. Adler, S. Afanasiev, et al., "Formation of dense partonic matter in relativistic nucleus-nucleus collisions at rhic: Experimental evaluation by the PHENIX collaboration," Nuclear Physics A, vol. 757, no. 1-2, pp. $184-283,2005$. First Three Years of Operation of RHIC.

[5] R. Bala, I. Bautista, J. Bielcikova, and A. Ortiz, "Heavy-ion physics at the LHC: Review of Run I results," Int. J. Mod. Phys., vol. E25, no. 07, p. 1642006, 2016, 1605.03939 .

[6] G. P. Salam, "Towards Jetography," Eur. Phys. J., vol. C67, pp. 637-686, 2010, 0906.1833.

[7] M. Connors, C. Nattrass, R. Reed, and S. Salur, "Review of Jet Measurements in Heavy Ion Collisions," 2017, 1705.01974.

[8] M. Cacciari, G. P. Salam, and G. Soyez, "FastJet User Manual," Eur. Phys. J., vol. C72, p. 1896, 2012, 1111.6097.

[9] D. J. Griffiths, Introduction to elementary particles; 2nd rev. version. Physics textbook, New York, NY: Wiley, 2008.

[10] F. Halzen and A. Martin, Quarks 6 Leptons: An introductory course in modern particle physics. New York, USA: John Wiley \& Sons, 1984.

[11] K. A. Olive et al., "Review of Particle Physics," Chin. Phys., vol. C38, p. 090001, 2014.

[12] H. Burkhardt and B. Pietrzyk, "Update of the hadronic contribution to the QED vacuum polarization," Phys. Lett., vol. B356, pp. 398-403, 1995. 
[13] CERN, "Standard model infographic - https://cds.cern.ch/record/1473657/ files/SMinfographic_image.png?subformat=," 2017. [Online; accessed 02December-2017].

[14] J. Letessier and J. Rafelski, Hadrons and Quark Gluon Plasma; new ed. Cambridge monographs on particle physics, nuclear physics, and cosmology, Cambridge: Cambridge Univ., 2005.

[15] "The Frontiers of Nuclear Science, A Long Range Plan," 2008, 0809.3137.

[16] J. D. Bjorken, "Highly Relativistic Nucleus-Nucleus Collisions: The Central Rapidity Region," Phys. Rev., vol. D27, pp. 140-151, 1983.

[17] P. Braun-Munzinger, "Towards the quark gluon plasma," Nucl. Phys., vol. A663, pp. 183-190, 2000, nucl-ex/9909014.

[18] F. Karsch, "Lattice results on qcd thermodynamics," Nuclear Physics A, vol. 698, no. 1, pp. 199 - 208, 2002. 15th Int. Conf. on Ultra-Relativistic Nucleus-Nucleus Collisions (Quark Matter 2001).

[19] T. K. Nayak, "Heavy Ions: Results from the Large Hadron Collider," Pramana, vol. 79, pp. 719-735, 2012, 1201.4264.

[20] F. Cooper and G. Frye, "Single-particle distribution in the hydrodynamic and statistical thermodynamic models of multiparticle production," Phys. Rev. D, vol. 10, pp. 186-189, Jul 1974.

[21] J.-Y. Ollitrault, "Relativistic hydrodynamics for heavy-ion collisions," European Journal of Physics, vol. 29, no. 2, p. 275, 2008.

[22] C. A. Salgado et al., "Proton-Nucleus Collisions at the LHC: Scientific Opportunities and Requirements," J. Phys., vol. G39, p. 015010, 2012, 1105.3919.

[23] M. L. Miller, K. Reygers, S. J. Sanders, and P. Steinberg, "Glauber modeling in high energy nuclear collisions," Ann. Rev. Nucl. Part. Sci., vol. 57, pp. 205-243, 2007, nucl-ex/0701025.

[24] B. Abelev et al., "Transverse momentum distribution and nuclear modification factor of charged particles in p-Pb collisions at $\sqrt{s_{\mathrm{NN}}}=5.02 \mathrm{TeV}$," Phys. Rev. Lett., vol. 110, no. 8 , p. $082302,2013,1210.4520$.

[25] J. Adam et al., "Measurement of charged jet production cross sections and nuclear modification in p-Pb collisions at $\sqrt{s_{\mathrm{NN}}}=5.02 \mathrm{TeV}$," Phys. Lett., vol. B749, pp. 6881, 2015, 1503.00681.

[26] V. Khachatryan et al., "Observation of Long-Range Near-Side Angular Correlations in Proton-Proton Collisions at the LHC," JHEP, vol. 09, p. 091, 2010, 1009.4122.

[27] S. Chatrchyan et al., "Observation of long-range near-side angular correlations in proton-lead collisions at the LHC," Phys. Lett., vol. B718, pp. 795-814, 2013, 1210.5482 . 
[28] B. Alver et al., "High transverse momentum triggered correlations over a large pseudorapidity acceptance in $\mathrm{Au}+\mathrm{Au}$ collisions at $\sqrt{s_{N N}}=200 \mathrm{GeV}$," Phys. Rev. Lett., vol. 104, p. 062301, 2010, 0903.2811.

[29] I. Vitev, J. T. Goldman, M. B. Johnson, and J. W. Qiu, "Open charm tomography of cold nuclear matter," Phys. Rev., vol. D74, p. 054010, 2006, hep-ph/0605200.

[30] E. Norrbin and T. Sjöstrand, "Production and hadronization of heavy quarks," The European Physical Journal C - Particles and Fields, vol. 17, no. 1, pp. 137-161.

[31] H. Abramowicz et al., "Measurement of charm fragmentation fractions in photoproduction at HERA," JHEP, vol. 09, p. 058, 2013, 1306.4862.

[32] S. Sakai, "Measurement of raa and v2 of electrons from heavy-flavour decays in pb-pb collisions at snn=2.76 tev with alice," Nuclear Physics A, vol. 904, pp. 661c - 664c, 2013. The Quark Matter 2012.

[33] J. Adam et al., "Measurement of electrons from heavy-flavour hadron decays in p- $\mathrm{Pb}$ collisions at $\sqrt{s_{\mathrm{NN}}}=5.02 \mathrm{TeV}, "$ Phys. Lett., vol. B754, pp. 81-93, 2016, 1509.07491.

[34] V. M. Abazov et al., "Measurement of the Top Quark Mass in Final States with Two Leptons," Phys. Rev., vol. D80, p. 092006, 2009, 0904.3195.

[35] J. E. Huth et al., "Toward a standardization of jet definitions," in 1990 DPF Summer Study on High-energy Physics: Research Directions for the Decade (Snowmass 90) Snowmass, Colorado, June 25-July 13, 1990, pp. 0134-136, 1990.

[36] V. Blobel, "An Unfolding method for high-energy physics experiments," in Advanced Statistical Techniques in Particle Physics. Proceedings, Conference, Durham, UK, March 18-22, 2002, pp. 258-267, 2002, hep-ex/0208022.

[37] G. Cowan, "A survey of unfolding methods for particle physics," Conf. Proc., vol. C0203181, pp. 248-257, 2002.

[38] G. de Barros, B. Fenton-Olsen, P. Jacobs, and M. Płoskoń, "Data-driven analysis methods for the measurement of reconstructed jets in heavy ion collisions at RHIC and LHC," Nuclear Physics A, vol. 910-911, pp. 314-318, 2013. Hard Probes 20125th International Conference on Hard and Electromagnetic Probes of High-Energy Nuclear Collisions.

[39] J. Adam et al., "Measurement of jet quenching with semi-inclusive hadron-jet distributions in central $\mathrm{Pb}-\mathrm{Pb}$ collisions at $\sqrt{s_{\mathrm{NN}}}=2.76 \mathrm{TeV}$," JHEP, vol. 09, p. 170, $2015,1506.03984$.

[40] L. Adamczyk, J. K. Adkins, G. Agakishiev, et al., "Measurements of jet quenching with semi-inclusive hadron + jet distributions in $\mathrm{Au}+\mathrm{Au}$ collisions at $\sqrt{s_{N N}}=200$ gev," Phys. Rev. C, vol. 96, p. 024905, Aug 2017.

[41] L. Adamczyk et al., "Jet-Hadron Correlations in $\sqrt{s_{N N}}=200 \mathrm{GeV} p+p$ and Central Au + Au Collisions," Phys. Rev. Lett., vol. 112, no. 12, p. 122301, 2014, 1302.6184.

[42] B. Abelev, J. Adam, D. Adamová, et al., "Measurement of electrons from semileptonic heavy-flavor hadron decays in $p p$ collisions at $\sqrt{s}=7$ TeV," Phys. Rev. D, vol. 86, p. 112007, Dec 2012. 
[43] ALICE Collaboration, "ALICE scheme figure - https://aliceinfo.cern.ch/ Figure/sites/aliceinfo.cern.ch.Figure/files/Figures/General/jthaeder/ 2012-Aug-02-ALICE_3D_v1_with_Text.jpg," 2015. [Online; Accessed 29-April2015].

[44] ALICE Collaboration, F. Carminati, P. Foka, et al., "Alice: Physics performance report, volume i," Journal of Physics G: Nuclear and Particle Physics, vol. 30, no. 11, p. $1517,2004$.

[45] P. Cortese, G. Dellacasa, R. Gemme, et al., "ALICE Electromagnetic Calorimeter Technical Design Report," Tech. Rep. CERN-LHCC-2008-014. ALICE-TDR14, CERN, Geneva, Aug 2008.

[46] ALICE Collaboration, K. Aamodt, A. A. Quintana, et al., "The alice experiment at the cern lhc," Journal of Instrumentation, vol. 3, no. 08, p. S08002, 2008.

[47] L. Evans and P. Bryant, "LHC Machine," JINST, vol. 3, p. S08001, 2008.

[48] CERN, "LHC complex - http://home.cern/about/accelerators," 2017. [Online; Accessed 10-October-2017].

[49] C. Lefèvre, "The CERN accelerator complex. Complexe des accélérateurs du CERN https://cds.cern.ch/record/1260465." [Online; Accessed 10-October-2017], Dec 2008.

[50] ALICE Collaboration, "Measurement of electrons from semileptonic heavy-flavour hadron decays in pp collisions at $\sqrt{7} t e v, " 2012,1205.5423$.

[51] ALICE Collaboration, B. Alessandro, F. Antinori, et al., "Alice: Physics performance report, volume ii," Journal of Physics G: Nuclear and Particle Physics, vol. 32, no. 10, p. $1295,2006$.

[52] P. Cortese et al., "ALICE technical design report on forward detectors: FMD, T0 and V0," 2004.

[53] ALICE Collaboration, "Performance of the ALICE VZero system," Journal of Instrumentation, vol. 8, no. 10, p. P10016, 2013.

[54] T. Awes, F. Obenshain, F. Plasil, et al., "A simple method of shower localization and identification in laterally segmented calorimeters," Nuclear Instruments and Methods in Physics Research Section A: Accelerators, Spectrometers, Detectors and Associated Equipment, vol. 311, no. 1, pp. 130 - 138, 1992.

[55] D. J. Fegan, "Gamma/hadron separation at tev energies," Journal of Physics G: Nuclear and Particle Physics, vol. 23, no. 9, p. 1013, 1997.

[56] C. Jahnke, Measurement of electrons from heavy-flavour hadron decays in $p$ - $P b$ collisions at $\sqrt{s_{N N}}=5.02 \mathrm{TeV}$ using TPC and EMCal detectors with ALICE at LHC. $\mathrm{PhD}$ thesis, Universidade de São Paulo, 2016.

[57] B. Lasiuk and T. Ullrich, "Star C ++ Class Library - User Guide and Reference Manual," 2001. 
[58] C. Grupen and B. Schwartz, Particle detectors. Cambridge, UK: Cambridge Univ. Pr., 2008.

[59] M. Cacciari, G. P. Salam, and G. Soyez, "The Catchment Area of Jets," JHEP, vol. 04, p. 005, 2008, 0802.1188.

[60] S. Chatrchyan et al., "Measurement of the underlying event activity in $p p$ collisions at $\sqrt{s}=0.9$ and $7 \mathrm{TeV}$ with the novel jet-area/median approach," JHEP, vol. 08, p. $130,2012,1207.2392$.

[61] T. Adye, "Unfolding algorithms and tests using RooUnfold," in Proceedings of the PHYSTAT 2011 Workshop, CERN, Geneva, Switzerland, January 2011, CERN2011-006, pp 313-318, pp. 313-318, 2011, 1105.1160.

[62] R. Barlow, "Systematic errors: Facts and fictions," in Advanced Statistical Techniques in Particle Physics. Proceedings, Conference, Durham, UK, March 18-22, 2002, pp. 134-144, 2002, hep-ex/0207026.

[63] M. Cacciari, M. Greco, and P. Nason, "The $p_{\mathrm{T}}$ spectrum in heavy flavor hadroproduction," JHEP, vol. 05, p. 007, 1998, hep-ph/9803400.

[64] M. Cacciari, S. Frixione, and P. Nason, "The $p_{\mathrm{T}}$ spectrum in heavy flavor photoproduction," JHEP, vol. 03, p. 006, 2001, hep-ph/0102134. 